Portland State University

PDXScholar

Summer 8-11-2015

\title{
Rational Design of Materials for the Protection of Outdoor Metalworks
}

Natasja Alexandria Swartz
Portland State University

Follow this and additional works at: https://pdxscholar.library.pdx.edu/open_access_etds

Part of the Art and Materials Conservation Commons, Metal and Jewelry Arts Commons, and the Polymer Chemistry Commons

Let us know how access to this document benefits you.

\section{Recommended Citation}

Swartz, Natasja Alexandria, "Rational Design of Materials for the Protection of Outdoor Metalworks" (2015). Dissertations and Theses. Paper 2488.

https://doi.org/10.15760/etd.2485

This Dissertation is brought to you for free and open access. It has been accepted for inclusion in Dissertations and Theses by an authorized administrator of PDXScholar. Please contact us if we can make this document more accessible: pdxscholar@pdx.edu. 
Rational Design of Materials for the Protection of Outdoor Metalworks

by

Natasja Alexandria Swartz

A dissertation submitted in partial fulfillment of the requirements for the degree of

Doctor of Philosophy

in

Chemistry

Dissertation Committee:

Tami Lasseter Clare, Chair

Dean Atkinson

Andrea Goforth

Andrew Rice

Portland State University

2015 


\begin{abstract}
Protective coatings are commonly used to protect culturally significant works, such as outdoor sculptures and architectural elements. Given the valuable nature of such metalworks, there is a surprising lack of environmentally sustainable coatings available for their conservation. High performance clear coatings are not developed or thoroughly tested for compatibility and longevity on outdoor sculptures. This can make the implementation of both methods and materials, no matter how promising in a lab, a significant hurdle for the conservation science community. This dissertation work initially aims to replace high-VOC formulations such as acrylic lacquers and waxes currently used as protective coatings for bronze with a waterborne coating by investigating the film formation differences between coating types. Such differences likely have implications for initial film barrier properties as well as long-term performance.
\end{abstract}

For coating any large-scale metal object, cost-effectiveness limits applicable coatings to commercially available resins with some minor adjustments. Additional requirements for protective coatings for artwork require they must also be transparent, reversible, easily applied and environmentally sustainable. The chemical and physical properties of polymeric coatings with nanoclays modifiers were investigated as they may offer superior weatherability and act as better barriers to water absorption than commonly used lacquers and waxes. This work ultimately finds that nanocomposites with poly(vinylidene fluoride) latex and chemically stabilized nanoclays significantly 
improved performance and may be a viable option in the protection of material cultural heritage. Protection of high value objects where aesthetics is also important, such as airplanes, buildings, and sculptures are among the possible applications for this research. 
This dissertation is dedicated to Tanya and Ineke Burkett, whose boundless love and generosity for this world have served me extensively as both inspiration and support in all of this life's endeavors.

How often people speak of art and science as though they were two entirely different things, with no interconnection. An artist is emotional, they think, and uses only his intuition; he sees all at once and has no need of reason. A scientist is cold, they think, and uses only his reason; he argues carefully step by step, and needs no imagination. That is all wrong. The true artist is quite rational as well as imaginative and knows what he is doing; if he does not, his art suffers. The true scientist is quite imaginative as well as rational, and sometimes leaps to solutions where reason can follow only slowly; if he does not, his science suffers. - Isaac Asimov, The Roving Mind 


\section{Acknowledgements}

To my advisor, Dr. Tami Lasseter Clare, who has given me more opportunities than can be listed in these pages, thank you for continuing to push me without making me fall. You opened a beautiful field of scientific research I never knew existed and I feel lucky to have worked at the intersection of chemistry and art with you these last many years. I sincerely appreciate your patience in listening to my every grand idea and your resolute confidence in my capabilities which has fundamentally shaped who I'll be forever.

To my esteemed committee as a whole, thank you for the time you have taken to read this dissertation and supportive feedback throughout my time in graduate school. To Dr. Dean Atkinson, thank you for always leaving your door open and being one of the best educators I have known. Your compassion for students is truly remarkable and it was a wonderful experience teaching with you. To Dr. Andrea Goforth, I will always be grateful for your generosity with your time and resources, especially the freedom you gave me to explore the capabilities of the XRD. To Dr. Andrew Rice, thank you for your kind encouragement and for taking the time to be the congenial Physicist on my committee.

To the many friends and colleagues in Portland, thank you for the comradery, the burritos in the Park Blocks, and the occasional family dinner. Most specifically I would like to recognize two exceptional women I was fortunate to work with, Katy Hosbein and Capri Price, for your contributions to the following work as well as being two of my favorite human beings. I would also like to thank Dr. Niles Lehman - not just 
for a bottomless well of support and a desk in his group's office - but also an email long ago that brought me to PSU. And Dr. David Peyton, Dr. Shankar Renanavare, and Dr. Mark Woods for years of encouragement and advice on things both science and life related.

Most importantly to my parents Tanya and Patrick and their partners Al and LeCarie, my siblings Cheyna, Shea, and Sierra, and the many members of the clans Swartz, Burkett, Heinrich, Letourneau, Bonin-Tustison, Nelson-Downs, Ma-Hayes and McClurg: there will never be enough words to express the gratitude I have for your unconditional support, sustaining hugs, and consistent reassurance, even when I haven't called in months.

And Rob - I'm so glad I met you here. 
I would also like to acknowledge the Institute of Museum of Library Services, the National Science Foundation and Portland State University for funding in support of the research contained in this dissertation: IMLS grant number LG-25-08-0071-08, NSF award numbers 1139230 and MRI DMR-0923572. Results reported in this dissertation have been disseminated in the following publications and presentations:

Swartz, N.A.; Price, C.A.; Clare, T.L., Effects of dispersed chemically-stabilized nanoclays in polymeric coatings on reducing corrosion of outdoor metalworks. 2015, in progress.

Swartz, N.A.; Clare, T.L., On the protective nature of wax coatings for culturally significant outdoor metalworks. J. Amer. Inst. Conserv., 2015, accepted.

Clare, T. L.; Swartz, N. A., Characterization of high performance protective coatings for use on culturally significant works. In Intelligent Coatings for Corrosion Control; Tiwari. A., Hihara L., Rawlins, J. Ed.; Butterworth-Heinemannr: November 2014. Hosbein, K.N.; Swartz, N.A.; Clare, T.L., Electrochemical identification and categorization of the protective quality of intact and damaged coatings. Electroanalysis. 2014, 26 (9), 1935-1944.

Swartz, N.A; Clare, T. L., Use of waterborne protective coatings with nanoclay additives to protect artwork. Presented paper at the $68^{\text {th }}$ ACS Northwest Regional Meeting, Corvallis, OR, July 21-24, 2013. 
Swartz, N. A.; Wood, K.; Clare, T. L. Spectroscopic analysis of aging fluoropolymer and acrylic clear coatings. Presented paper in Methods and Testing, Proceedings of the American Coatings Association, Indiannapolis, IN, 2012.

Swartz, N. A.; Wood, K. A.; Clare, T. L., Characterizing and improving performance properties of thin solid films produced by weatherable waterborne colloidal suspensions on bronze substrates. Prog. Org. Coat. 2012, 75 (3), 215-223.

Swartz, N.A., Hosbein, K., England, A., Clare, T.L. Characterization and in situ monitoring of protective coatings for culturally significant outdoor works. Contributed poster at Gordon Seminar on Scientific Methods in Cultural Heritage Research, West Dover, VT, 2012.

Swartz, N. A.; Clare, T. L. Reducing water sensitivity in waterborne PVDF-latex nanocomposite coatings by controlling long-range order of a synthetic nanoclay. Presented paper in Preprints of ACS Division PMSE, 243rd National Meeting of the American Chemical Society, San Diego, CA, 2012.

Swartz, N. A.; Clare, T. L., Understanding the differences in film formation mechanisms of two comparable solvent-based and waterborne coatings on bronze substrates by electrochemical impedance spectroscopy. Electrochim. Acta 2012, 62, 199-206.

Swartz, N.A.; Clare, T. L., Fundamental analysis of weatherable polymer films. Presented paper at the $66^{\text {th }}$ ACS Northwest Regional Meeting, Portland, OR, June 2629, 2011. 


\section{Table of Contents}

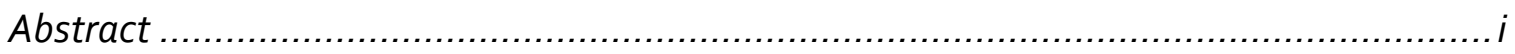

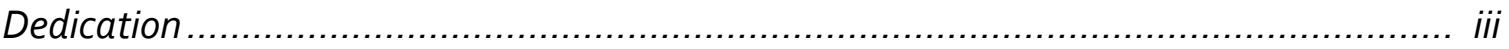

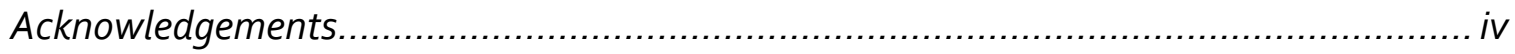

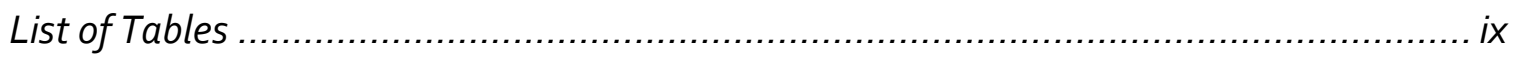

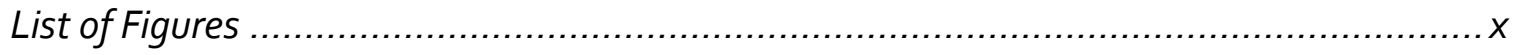

Glossary and List of Abbreviations ....................................................... xiii

$1 \quad A$ (very) brief history of metals and their coatings .......................................... 1

2 On the protective nature of wax coatings for culturally significant outdoor metalworks: microstructural flaws, oxidative changes, and barrier properties.......... 21

3 Understanding the differences in film formation mechanisms of two comparable solvent-based and waterborne coatings on bronze substrates by electrochemical impedance spectroscopy

4 Characterizing and improving performance properties of thin solid films produced by weatherable waterborne colloidal suspensions on bronze substrates....

5 Investigating the protective lifetime of novel waterborne and traditional solventbased coatings for the protection of outdoor metalworks.

6 Reducing corrosion of outdoor metalworks using dispersed chemically-stabilized nano-clays in PVDF latex coatings ...................................................... 152

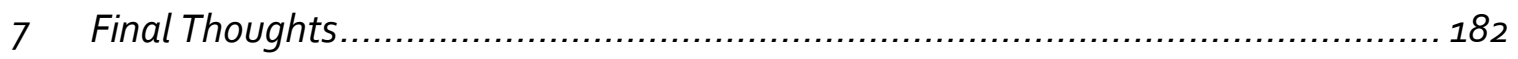

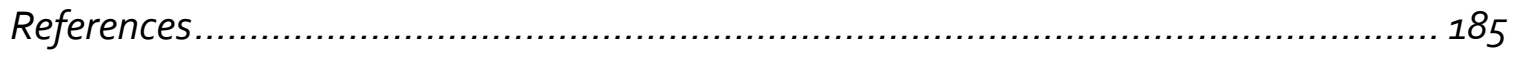

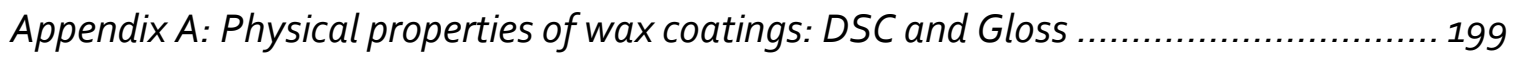

Appendix B: Micrographs and photographs of weathered wax samples ......................200

Appendix C: Corrosion products by accelerated and outdoor weathering..................... 202

Appendix D: Detailed analysis of impedance spectra of wax coated substrates .............205

Appendix E: Impedance derivative analysis on steel and patinated bronze ................... 208

Appendix F: Additional surface characterization of clays, PNCs, and their properties .... 220 
Table 2.1. TF-XRD and EIS of waxes before and after annealing

Table 2.2. Characterization of significant IR absorption peaks in waxes

Table 2.3. ${ }^{1} \mathrm{H}$ NMR character table of S. Florida-weathered Butcher's wax on bronze

Table 2.4. Peak area ratio from $\mu$-FTIR and ${ }^{1} \mathrm{H}$ NMR data of waxes

Table 2.5. TF-XRD characterization and film thickness gauge measurements of wax remaining after weathering

Table 4.1 Water uptake in topcoats with varying percentages of DPM

101

Table 5.1. Properties of polymers utilized in this study

Table 5.2. Gloss and film thickness values of coated bronze substrates shown in \% retention (Final Value/Initial Value $\times 100 \%$ )

Table 5.3. Gloss and film thickness values of coated steel substrates shown in \% retention (Final Value/Initial Value $\times 100 \%$ )

Table 6.1 Particle size and stability in aqueous and colloidal suspensions

Table 6.2 Barrier properties of the films before and after 4250 hours QUV-B

Table 6.3 Gloss and thickness of the films before and after 4000 hours QUV-B 


\section{List of Figures}

Page \#

$\begin{array}{ll}\text { Figure } 1.1 \text { Conditions of accelerated (QUV) weathering } & 11\end{array}$

Figure 1.2 Example of general mechanisms for photooxidation 13

$\begin{array}{ll}\text { Figure 1.3 Structure of Laponite } & 15\end{array}$

Figure 1.4 Torturous path for molecule to substrate in PNCs 18

Figure 1.5 Cross section of modified clay (small disks) in polymer $\quad 18$ suspension (spheres)

Figure 2.1 Schematic of a waxed metal substrate at various stages of 25 deterioration

Figure 2.2 Results of Blind Study related to wax coatings 33

Figure 2.3 Spectral changes upon annealing of waxes $\quad 36$

Figure 2.4 Infrared spectra from waxed panels before and after $\quad 40$ weathering

Figure $2.5^{1} \mathrm{H}$ NMR spectra from waxed panels before and after $\quad 41$ weathering

Figure 2.6 EIS Bode plots from all waxed samples after weathering 51

Figure 2.7 Extracted circuit elements from fitting EEC models to EIS 53 spectra of waxes

$\begin{array}{ll}\text { Figure 3.1 Drying time schematic of solvent-based and waterborne } & 62\end{array}$ coating systems

Figure 3.2 Differential scanning calorimetry thermograms of waterborne 63 and solvent-based polymers

Figure 3.3 Electrical impedance spectrum of BTA layer alone after 68 pretreatment of bronze

Figure 3.4 EIS Bode plots of solvent-based film formation 70

Figure 3.5 EIS Bode plot of waterborne film formation 72

Figure 3.6 Water uptake of solvent-based and waterborne films of 75 increasing thickness

Figure 3.7 Change in |Z| of solvent-based and waterborne films 
Figure 3.8 Bode plot of impedance changes of solvent-based and waterborne films

Figure 4.1 Exploded view of vinyl and embedded gold electrodes in coating layers on substrates

Figure 4.2 EIS of Kynar ARC or B-44 coated bronze coupon and ECC models fit to experimental data

Figure 4.3 Water-whitening of layered films studied by FT-IR 98

Figure $4.4 \mathrm{Immersion}$ studies of layered coatings with varying coalescent (DPM)

Figure 4.5 Electrochemical Impedance Spectra of RC-10206 with varying 100 concentrations of DPM

Figure 4.6 Grayscale values found during immersion studies of vinyl with 104 RC-10206

Figure 4.7 Improved barrier properties of coatings with longer drying 107 times and annealing

Scheme 5.1 Representations of polymers in coatings investigated 116

Figure 5.1 An overview of long-term barrier properties of polymeric 121 coatings

Figure 5.2 Results of exit survey from conservation Blind Study 127

Figure 5.3 Averaged EIS Bode plots of coated bronze and steel samples 129 by blind study participants

Figure 5.4 Impedance derivatives during weathering of plain bronze 131 panels coated with acrylic resins

Figure 5.5 FTIR of acrylic coatings on bronze before and after weathering 135

Figure 5.6 Derivative analysis of impedance to investigate the effects of 139 additives in acrylics

Figure 5.7 Derivative analysis of impedance for Kynar ARC and FMA on $\quad 142$ bronze

Figure 5.8 FTIR of PVDF coatings on bronze before and after weathering $\quad 146$

Figure 5.9 DSC thermograms of B44, WL81, and Kynar ARC 149 
Figure 6.1 Laponite schematic $\quad 155$

Figure 6.2 XPS of modified Laponite clay $\quad 163$

Figure 6.3 Raman spectra of the hydroxyl and aliphatic regions of nano- $\quad 165$ clays

Figure 6.4 SAXS models of modified nano-clays in PVDF Latex $\quad 167$

Figure 6.5 Water-whitening of nanocomposites 168

Figure 6.6 Mass transport (diffusion) profiles of nanocomposites $\quad 169$

Figure 6.7 Impedance analysis of the films with and without nano-clays 173

Figure 6.8 FTIR-ATR spectra of nanocomposite coated bronze before $\quad 177$ and after weathering by QUV 


\section{Glossary and List of Abbreviations}

Additive

Adhesion

Amorphous

APTMS

Binder

Blistering

Bode plot
A material used to confer a specific property to a coating; typically added in less than $1 \%$ weight additive/total weight of the coating $(1 \% \mathrm{w} / \mathrm{w})$

Measure of the strength of interaction between two materials in close contact.

Non-crystalline phase of a material. In polymers this phase is present as a continuous phase of disordered polymer chains.

Silanization agent 3-acetoxypropyltrimethoxysilane

The medium or matrix in which a discrete second-phase is enclosed, which is typically the polymer part of a coating, while the second-phase is the pigment. The binder is also called the continuous phase.

Formation of small to large bubbles under a coating at the coating-metal interface. Blistering causes adhesion failure and blisters continue to grow with water exposure due to osmotic effects

Output of frequency-dependent EIS data in the form of magnitude (|Z|) and phase $(\Phi)$ 
Bronze

BTA

Bulk metal

Butcher's Wax

Capacitance

Chemical changes

Coalescence

Composite
An alloy consisting primarily of copper and tin but may contain lead, silicon, zinc, with traces of aluminum, manganese or nickel

Abbreviation for benzotriazole. A common corrosion inhibitor for copper alloys in conservation, that forms a thin film with surface copper atoms

The continuous alloy phase of a metal substrate. Serves as the working electrode during EIS

A hard amber paste wax composed of iso- and n-paraffinic microcrystalline and natural carnauba wax

Amount of separated electric charge than can be stored in a dielectric material sandwiched between two conductors

Any change that results in the formation of new chemical substances from the making (synthesis) or breaking (decomposition) of bonds

The action of fusion of polymer particles in water-borne paint to produce a continuous film on a substrate.

A material made up of multiple phases within a single matrix leading to a material with different properties than those of 
the individual constituents

Conservation

Treatment to preservation of the original form of a historic or artistic object in such a manner that minimizes the need for future intervention. Such treatments are, ideally, minimally visible so that the object appears as if it was untreated.

Constant phase

A circuit element that models an imperfect (leaky) capacitor, element most notably with a frequency-dependent phase element where $1 \leq \alpha \leq 0$

Corrosion The deterioration of a substance due to reactions with its environment

Corrosion inhibitor A substance which, when added in a small concentration, effectively reduces the corrosion rate of a metal exposed to a certain environment

Corrosion products The material produced from a corrosion reaction, typically a metal oxide

Cross-linking The process by which a low molecular weight functional polymer is converted to an infusible, insoluble, three dimensional polymer network by the chemical reactions.

Material Cultural The legacy of physical artefacts and intangible attributes of a 
Heritage

Delamination

Diffusion

Dry film thickness

Electrical double

layer

Electrical

equivalent circuit

model (EEC)

Electrochemical

impedance group or society that are inherited from past generations, maintained in the present and bestowed for the benefit of future generations

The loss adhesion at the interface of two materials. In this work, that interface is between a coating and substrate or two different coatings.

The net transport of mass (molecules, atoms, or their components) from a region of high concentration to a region of low concentration, typically in or creating a gradient.

The thickness of a film, such as a coating, as measured above the substrate after the film dries. The film itself can be one or multiple layers.

A diffuse aggregation of positive and negative electric charges organized on a surface or surrounding a particle

A theoretical circuit that acts as a physicochemical representation of the coated metal system, in EIS the circuit model is used for fitting to the acquired data

A non-destructive analytical technique that measures the dielectric properties of a material by applying a potential at the 
spectroscopy

Farad (F)

Film formation

Flash-rust

formulation

FOTES

Free radical working electrode and monitoring the propagation of current

at the counter electrode over a range of frequencies

Unit of capacitance, equal to one coulomb per volt

The process by which a liquid coating of low viscosity is applied to a substrate and cures into a solid, high-molecular weight polymer film possessing intended properties. The solvent used to disperse the polymer affects the length of time the film takes to cure.

Rapid corrosion that occurs nearly instantaneously when metals are exposed to a corrosive environment such as water (or humid air). Flash rust often occurs when a waterborne coating is directly applied to an iron or steel substrate. The corrosion products typically migrate into the coating and stain the film.

The wet mixture of a coating which includes the polymer, solvent, and additives in specific percentages.

Silanization agent (tridecafluoro-1,1,2,2-tetrahydrooctyl) triethoxysilane An atom or molecule that contains one or more unpaired 
electrons making it highly reactive

Glass transition

temperature

Gloss

Grazing-Incidence

X-Ray Diffraction

$(G I X R D)$

HALS

Impedance

Incralac
The temperature or range over which segments of an amorphous polymer achieve sufficient mobility to transform from a vitrified solid (glass) to a liquid (for non-cross-linked polymers) or to a rubber (for cross-linked polymers) Reflectivity of a light on a surface, measured in \% Reflectance A surface-sensitive technique that uses an X-ray beam to obtain a diffraction pattern used for phase identification of a bulk material. The beam is aligned at a very small grazing angle $(<0.92 \theta)$ on the surface of the film Acronym for Hindered-Amine Light Stabilizer The effective resistance of an electric circuit or component to an $\mathrm{AC}$ potential from the combination of ohmic resistance and reactance. The magnitude of impedance is given in ohms $\cdot \mathrm{cm}^{2}$ and is abbreviated with the letter " $Z$ "

A solvent-based, clear, high-gloss, air-dry coating formulated by StanChem for copper-base alloys. The formulation includes Paraloid B-44 as the resin along with BTA, HALS, and soybean oil 
Interstitial space

Kynar ARC

Kynar FMA

Lacquer

Latex

Low-carbon steel

Mass-transport
The 'empty' space in an amorphous polymer. Also called the 'free volume' as it's the difference between the total volume of the sample and the volume occupied by the polymer segments

A waterborne, fluorinated-acrylic blended latex resin

composed of 70\% PVDF and 30\% PMMA-co-PEA,

manufactured by Arkema

A waterborne, fluorinated-acrylic blended latex resin

composed of 50\% PVDF and 50\% PMMA-co-PEA, manufactured by Arkema

A coating that dries by the evaporation of a solvent

An aqueous dispersion of colloidal polymers that dries into a flexible film. Waterborne coatings are referred to as a 'latex' when in their wet form in a jar.

A softer, ductile iron alloy that is possible to machine or weld

The diffusive, convective or migratory transport of chemical species within a physical system. This term excludes the movement of electrons.

Mechanical Changes that result in the alteration of the physical properties changes (e.g. adhesion, tensile strength, softness) of a material. 
Microcrystalline

A substance that contains crystals whose domain size is on the micron scale

Microstructure

Micron-sized structures

Mineral spirits

A solvent composed of mostly paraffinic hydrocarbons with a boiling point range of $150-190^{\circ} \mathrm{C}$

Circuit Model

An organization of electrical elements (e.g. resistors and capacitors) that standardizes how the individual elements relate to each other (e.g. in series or in parallel). Such models are used to fit data to gain insight into the mathematical value of each element in the model.

Nano-clay

Layered silicate with at least two-dimensions in the nano-scale $\left(10^{-9}\right.$ meters $)$

Nanocomposite A multiphase solid material where at least one phase has a dimension that is less than $100 \mathrm{~nm}$ in scale

Ohms A unit of electrical resistance that is equal to one volt per ampere

Oxide A chemical compound that contains at least one oxygen atom and one other element in its chemical or oxidized form

Paraloid B44 A solvent-based polymer resin composed primarily of PMMA 
and PEA, manufactured by Dow

Passivation

Typically the formation of a metal oxide layer to produce a protective barrier against further corrosion

Patina

Metal-oxide passivating layer formed by natural and/or artificial methods to produce a homogeneous surface appearance

Pc

Phosphoryl choline tetrahydrate

PEA

Poly(ethyl acrylate)

Peptisation

Neutralization of charged groups on a polymer to induce water-dispersibility

Percent solids

The percent ratio of the volume or weight of non-volatile material in paint to its total wet volume, expressed as a percentage

Permeability Ability of oxygen, water vapor, and ions to migrate through a polymer film

Phase angle The angular component $(\Phi)$ of the impedance vector

Photo-oxidation Degradation of a polymer due to hydrogen abstraction reactions with peroxy radicals which are generated photochemically by reaction with adventitious radicals with 
oxygen

Pinholes

Small holes in a coating having an aspect ratio similar to a needle that often result in exposure of small circles of the substrate

PMMA

Poly(methyl methacrylate)

PnBM

Poly(n-butyl methacrylate)

PNC

Polymer Nanocomposite

Protective lifetime The time over which a particular coating acts to protect a metal against corrosion

PS

Polystyrene

Public works

Any constructed object that augments the physical infrastructure

PVDF

Poly(vinylidene fluoride)

QUV

A proprietary testing cabinet in which a painted panel is exposed to UV-B or UV-A radiation and/or condensation

Renaissance wax A soft paste wax composed of a refined mixture of microcrystalline iso-paraffins blended with mineral spirits

Resin

A material used as the binder or continuous phase in a coating 
Resistance

Rheology

Rhoplex WL81

Rust

Salt-spray test

Small-Angle

X-Ray Scattering

(SAXS)

Soaking test

Solvent-based

coating

Thermoplastic

Thin-Film X-Ray
Opposition to the flow of current employed to protect a circuit

The property of flow in given a material

A waterborne latex resin composed primarily of $\mathrm{PnBM}$ and PS, manufactured by Dow

Corrosion that occurs when oxygen combines with metal at an atomic level

A test where a coated panel is exposed to an atmosphere of saturated salt-spray that is intended to challenge the anticorrosive properties of the coating.

A non-destructive method that uses the scattering of a focused X-ray beam for investigating the geometry and spacing of structures between 1-200 $\mathrm{nm}$ in size.

A test where a panel is immersed in a liquid electrolyte and the impedance of the panel is measured over time.

A coating whose viscosity is adjusted by the use of a fastevaporating organic solvent

Polymers that are not cross-linked and can be softened by heating or by contact with suitable solvents

A technique that uses an X-ray beam to obtain a diffraction 
Diffraction

(TFXRD)

Total Impedance

$(|\mathrm{Z}| 0.1 \mathrm{~Hz})$

VOC

Waterborne

coating

Water-uptake

Wax

Weathering,

accelerated

(QUV)

Weathering,

outdoor

(PDX or S.FL)

Water whitening pattern used for phase identification of a bulk material, with the beam aligned and focused at the top-surface of a film.

Magnitude of impedance in $\Omega \cdot \mathrm{cm}^{2}$ derived from a Bode Plot at the lowest frequency measured, typically $0.1 \mathrm{~Hz}$.

Volatile Organic Components. Any organic component with high volatility so that is vaporized into to the atmosphere under the normal condition of use.

A coating whose viscosity is adjusted by the use of water

Type of mass-transport where water percolates into a material.

Mixtures of high-molecular weight alkanes with chain-lengths of 20-50 carbon units.

Process of aging polymeric coatings in an artificial weathering chamber (QUV) to monitor effects of UV-B and condensation cycles. Exposure times are measured in hours.

Process of aging polymeric coatings outdoors on a $45^{\circ}$ angled, southern facing rack. Exposure times are measured in months.

Phenomenon observed in coatings caused by water-filled 
inclusions that are large enough to scatter light.
Wide-angle X-Ray A technique that uses an X-ray beam to obtain a diffraction
Diffraction (WAXD) pattern used for phase identification of a bulk material.
X-Ray A surface-sensitive technique that uses an X-ray beam to
Photoelectron measure the kinetic energy of escaping electrons of a material
Spectroscopy (XPS) to provide an elemental composition.

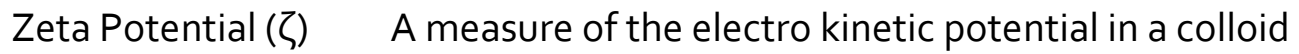 suspension related to the magnitude of electrostatic repulsion or attraction between particles.

$\mu$-Fourier An analytical technique that uses a microscope attached to an Transform Infrared infrared spectrometer to select portions of a solid or liquid spectroscopy sample to obtain an IR vibrational spectrum. Radiation $(\mu-\mathrm{FTIR})$ absorbed by the sample produces peaks at characteristic frequencies indicative of the types of functional groups present.

$\mu$-Raman An analytical technique that uses a microscope attached to a spectroscopy laser and Raman detector to select portions of a solid or liquid ( $\mu$-Raman) sample to obtain a Raman vibrational spectrum. The resulting spectrum contains peaks at characteristic frequencies indicative of the types of functional groups and bonds present. 


\section{A (very) brief history of metals and their coatings}

Crumbling infrastructure affects nearly every single human being on the planet. ${ }^{1} \mathrm{~A}$ substantial amount of energy is required to extract metals from ores, and in Earth's current atmosphere, as soon as the reduced metals are isolated they begin to convert back to a more stable oxidized state. Metallurgy comprises one of the earliest known displays of human creation and tangible cultural identity. Copper and iron alloys more specifically constitute a large portion of humanity's metallic cultural heritage and ultimately its surviving infrastructure. As such, corrosion of metals since the Bronze and Iron Ages has constituted a significant challenge to most societies due largely to the massive financial loss. Varying reports show corrosion of metals as a global economic problem that alone costs the U.S. about $\$ 276$ billion per year, the E.U. $€ 200$ billion and ultimately $\$ 3-4$ trillion per year globally, which is approximately $4.2 \%$ of the world's GDP. ${ }^{2}$ Improvements in protective coatings of metals have been estimated to save millions of dollars alone with regard to reduced costs of metal materials (due to increased service life and subsequent conservation of resources). ${ }^{3}$

Protective coatings for metals have been used and developed for thousands of years, with early observations of corrosion and conservation efforts with oils, fats, and natural resins documented by Pliny the Elder. ${ }^{4}$ Changes in the environment, both natural and anthropogenic, have required subsequent adaptations to the materials used to protect metals from their surroundings which have an increasing quantity of pollution, heat, and ozone. While early protective coatings were naturally derived from 
tar, exudates, and fat, current protective coatings are almost entirely synthetic. For decades, the $\$ 100$ billion coating industry has been creating materials specifically tailored to their applicable substrates and intended environment. Given their highvalue, conservation of culturally significant metalworks requires a level of extensive testing that is not cost-effective for companies and is impractical for most museums given recent cuts in institutional funding. Ultimately this work uses recent industrial advances in waterborne fluorinated polymers in combination with a uniquely modified nano-clay filler to provide the conservation science community with a new environmentally friendly coating for the protection of high value and monumental metal objects where aesthetics are important, such as airplanes, buildings, and sculptures.

\subsection{Mechanisms for Atmospheric Corrosion of Metals}

Corrosion occurs when a metal passes from the elementary state (often in an alloy) to a combined state and generally occurs via an anodic reaction between a metal and its environment as shown in equation 1.1:

$$
M \rightarrow M^{n+}+n e^{-}
$$

where $M$ is the metal undergoing dissolution, $n$ the number of electrons and $e^{-}$the electrons lost during oxidation. Since metals are thermodynamically unstable under oxidizing conditions, they immediately undergo corrosion reactions unless protected from their surrounding environment. Typical oxidation reactions for metals undergoing outdoor weathering occur under aqueous conditions by the accumulation of 
atmospheric water and salts on their surface. Many metals and alloys, such as copper and bronze, can form oxide films (patinas) on their surface which will slow the dissolution of the underlying metal. Though these films are generally passivating and can provide a small amount of protection to the underlying substrate, they are not sufficient for long term outdoor exposure. ${ }^{5-7}$ Also, if atmospheric salts containing aggressive Halides $\left(\mathrm{Cl}^{-}, \mathrm{Br}\right)$ or conjugate bases $\left(\mathrm{ClO}_{4}^{-}, \mathrm{SO}_{4}{ }^{2-}\right)$ are present, they can inhibit formation of a stable patina through competitive adsorption and instead promote the onset of local degradation and eventually pitting ${ }^{8}$ Since oxidizing a metal decreases mechanical strength, dissolution of the elemental metal can weaken and cause irreversible damage to structures if left unimpeded. Thus for objects of highvalue that are difficult or impossible to replace, conditioning of the metal through means of a protective organic coating is necessary.

\subsection{Mechanisms for Barrier Protection of Metals}

Protective coatings work by inserting a high resistance material to insulate the anodic and cathodic areas of a corrosion reaction on the metal substrate. Materials that make excellent protective coatings have compatible surface energy with the metal resulting in good adhesion and excellent barrier properties that slow the transport of water and ions through the film. One of the most common modes of coating failure is blistering, which occurs through a buildup of osmotic pressure from accumulation of contaminants or high ionic strength species at the coating metal interface. Unlike water, UV radiation, gases and vapors are all small enough to pass easily through the 
organic film. The more common mode of failure in this study was from mechanical stress induced by weathering and degradation of the polymer that resulted in cracking of the film.

For older coatings, reasons for their failure were unknown as missing mechanistic information was often compensated for by practical and anecdotal experience. Advances in analytical methods have begun to quantitatively demonstrate a clear need for more durable coatings for outdoor applications on high-value metalwork that exceed the shorter lifetime of current solvent-based systems and waxes. ${ }^{9-14}$ In addition, legislative restrictions and costs of solvents have also provided a motivation to reduce VOC (volatile organic compound) levels in coatings to develop environmentally friendly waterborne coatings systems. VOC's are generally defined as organic compounds emitted as a gas from a solid or liquid and are restricted due to their adverse effects on public health and the environment. ${ }^{15}$ They contribute directly to: (1) ozone holes, (2) summer smog, (3) climate change, (4) acid rain, and (5) carcinogen exposure. ${ }^{16}$ Hence state and federal environmental regulations have positioned waterborne coatings to replace solvent-based coatings in many applications. However, the greater susceptibility of many waterborne coatings to a reduction in their barrier properties upon exposure to water, (with various negative side effects including blistering and water-whitening) poses a significant hurdle for the possible future role of waterborne coatings as environmentally-friendlier alternatives for the corrosion protection of metals. ${ }^{17}$ In 1954, Wheeler et al. stated the need for a compromise in aqueous polymer dispersions between stability and performance, a 
tension which remains to this day. ${ }^{18}$ Clear coatings are particularly difficult because pigments and fillers typically can't be added to compensate for the weaknesses in waterborne polymer materials.

For use in conservation of cultural heritage, a combination of protective and aesthetic requirements becomes difficult to meet with clear coatings. A unique code of ethics $^{19}$ exists which fundamentally require that treatments by conservators alter the aesthetics of an object as little as possible. This entails that all coatings included in this work are optically transparent, reversible by means of solvent as opposed to ablation and have low gloss (as measured by \% reflectance). Furthermore, in today's economic climates and reduced public funding, conservation materials need to be inexpensive, available, as well as tailored to their unique requirements. ${ }^{19}$ We have referred to this set of standards in combination with our own in Chapter 5 as TRACE, which stands for Transparent, Reversible, Applied, Commercially available, and Environmentally friendly. For these applications and the standards set by others, ${ }^{20-21}$ beyond exceptional weathering performance, the waterborne PVDF nanocomposites developed in Chapter 6 of this dissertation (1) had low gloss and minimized change to the appearance of the metal, (2) remained soluble and reversible in addition to (3) flexible after weathering to minimize cracking, (4) and chemically and physically stable to retain optical and mechanical properties.

Metal protection is not only gained by the use of a protective coating, but optimized when the components of the formulation are targeted to the substrate metal and subsequent environment. This can be done by additives to increase adhesion, 
inhibit corrosion, and modify rheology to speed drying or slow mass transport through films. Additives in this research were added to coating formulations to enhance longterm protection of the metal surface and included corrosion inhibitors, flash-rust inhibitors, hindered amine light stabilizers (HALS) and nano-clays.

\subsection{Water as a solvent in organic coatings}

Ideally, the polymer resins of organic coatings should be hydrophobic to prevent water sorption as their primary role is to prevent the passage of gases, vapors and ions through a film to the metal substrate. However, hydrophobic polymers are not normally stable as waterborne suspensions, and so require the addition of hydrophilic additives, or modification of the polymer to include hydrophilic components, as a means of stabilizing these suspensions. ${ }^{22-23}$ The technical challenges for wide-spread use of waterborne coatings lie not only in the development of UV- and oxidationresistant polymers, but also in producing continuous, defect-free films from the aqueous phase; because, unlike solvent-based coatings, aqueous colloidal suspensions contain a number of chemicals added into the coating formulation to aid in the application and drying process (e.g. surfactants, defoamers, coalescing agents and rheology modifiers). ${ }^{24-27}$ In addition to formulation additives, application of waterborne coatings on reactive surfaces such as metal alloys of copper and iron require the use of flash-rust inhibitors, either as a pre-treatment or as a coating additive. ${ }^{28-31}$ 


\subsubsection{Film formation}

The mechanisms of film formation from solvent-based versus waterborne coatings are significantly different and have been examined through a variety of means in multiple fields. ${ }^{32}$ Although intermediate phases of latex film formation remain a point of contention among many, ${ }^{32-36}$ two-stages typically exist for solvent-based polymers and three for waterborne emulsions, which are extensively discussed in Chapter 3. The coalescing agents typically used in waterborne polymer emulsions have low vapor pressures (e.g. 2-butoxyethanol, vapor pressure $=79.9 \mathrm{~Pa}$ at $20^{\circ} \mathrm{C}$ ) compared to organic solvents used in solvent-based coatings (e.g. tolvene, vapor pressure $=2.93 \mathrm{kPa}$ at $20^{\circ} \mathrm{C}$ ), meaning they diffuse more slowly through the polymer film and are retained within the film for longer periods of time.

We found the differences in film formation between solvent and waterborne polymer films, e.g. slower drying times, to be critical for understanding performance differences in current coatings technology. ${ }^{37}$ Chapter 3 uses electrochemical impedance spectroscopy (EIS) as a form of rapid assessment of the changes in barrier properties during formation of acrylic films cast from a solvent-based (dissolved polymer) or a waterborne (polymer emulsion) onto bronze substrates. Mass loss curves during film drying were compared to EIS data and the data were used to correlate physical changes with electrochemical changes of the two film types. The electrochemical changes observed during drying were fit using circuit models and support theoretical physical changes in film structure. Aging studies in our lab and others ${ }^{38-39}$ have shown an initial increase in barrier properties of water borne films after they have dried for thirty days 
and longer, ${ }^{40,41}$ a phenomenon which has not been observed with solvent-based films. (However, with prolonged weathering, the polymer chains of both types break down and the barrier properties of the films still decrease. $)^{40-42}$ Since we only observe this change upon extended drying in waterborne films, we attributed those latent increases in impedance are likely due to trapped additives that slowly diffused out of the film over a period of weeks.

\subsubsection{Swelling and uptake mechanisms}

Water sorption into a polymer film is dependent on a multitude of factors including: attachment/adhesion to the substrate, surface roughness, porosity and degree of film coalescence. ${ }^{43}$ Waterborne coatings have an increased sensitivity to water when immersed or exposed due to hydrophilic additives that aid predominately in emulsion stabilization. The causes of whitening in organic thin film coatings have been attributed in large part to the additives to the formulations and not from the polymer resins. ${ }^{44}$ The first documentation of water whitening implicated emulsifiers as the cause and discussed possible interactions of residual additives and the polymer network which lead to hydrophilic inclusions which swelled with water and caused increased light

scattering. ${ }^{18}$ Investigations of many surfactants in acrylic-based latex films found that all formulations whitened upon immersion in water due to hydrated surfactant reservoirs that swell and scatter light, creating a "white and opaque film". ${ }^{44}$ Water uptake, and the visual whitening effect that may follow, is increased by the hydrophilic nature of waterborne coatings, and by sub-microscopic channels in the polymer film 
caused by the evaporation of slow co-solvents. ${ }^{45}$ One description of surfactant effects details a worst-case scenario that could create a hydrophilic pathway to the metal where surfactant accumulation around particle boundaries created hydrophilic pockets to trap water and speed water transport. ${ }^{46}$

\subsubsection{Acrylic and fluorpolymer emulsions}

The ability of coatings to prevent corrosion also depends upon the chemistry and durability of the polymer, especially its ability to resist degradation due to UV light exposure. ${ }^{42,}{ }^{47-48}$ Fluorinated polymer hybrid blends based on poly(vinylidene fluoride) (PVDF) are among the most UV-resistant resin systems used today in commercial coatings for metals, with protective properties lasting in many cases for more than thirty years of outdoor exposure, as described by Wood et al. and others. ${ }^{11,47-49}$ Since their debut in the 1960s, PVDF-containing coatings have primarily been supplied as a non-aqueous dispersion or solution coatings, often at high VOC levels. Recent advances have produced waterborne (latex) hybrid dispersions with high PVDF resin content and the performance of these coatings continues to improve. ${ }^{50-52}$ Commercial products of this type, such as KYNAR Aquatec ${ }^{\circledR}$ ARC and FMA, contain $70 \%$ or $50 \%$ PVDF on total resin weight, with the remainder of the resin being acrylics. ${ }^{11,47}$ These proportions are similar to PVDF:acrylic ratios used typically in traditional solvent PVDF-containing coatings. Like solvent PVDF coatings, waterborne PVDF coatings of this type have been shown to offer superior barrier properties, after exposure to UV light, compared to solvent-based $100 \%$ acrylic coatings. ${ }^{11}$ Poly(methyl methacrylate) is also known to be 
more resistant to UV degradation and is frequently co-polymerized with ethyl acrylate to improve mechanical properties of the latex.

Because of their relatively inert chemistry (which confers resistance to oxidative degradation, and other mechanisms affecting coating durability), 100\% PVDF coatings do not provide good adhesion to metals, compared to acrylic resins such as Paraloid ${ }^{\circledR}$ B-44, a methyl methacrylate/ethyl acrylate copolymer. ${ }^{53}$ We circumvent this adhesion problem by using an acrylic basecoat (for good adhesion) combined with a waterborne PVDF-based topcoat (for good weatherability). However, we have found a remaining challenge with some prototype systems of this type: the clear films are susceptible to whitening upon exposure to water. This is particularly problematic in outdoor applications for aesthetic reasons, and as possible indications of poor short and longterm performance. Early work detailed in Chapter 4 focuses on understanding the mechanism of water whitening, using spectroscopic and electrochemical impedance tools, in order to identify ways to reduce or prevent it. ${ }^{37}$

\subsection{Design and testing of federally compliant high-performance waterborne coatings}

One of the drivers of industrial coatings development is reducing the cost of materials, while not losing (or perhaps even improving) performance. Current high-performance coatings used in the conservation of cultural heritage have a lifetime expectancy of one year for waxes and two to three years for solvent-based coatings before stripping and reapplication. ${ }^{20,54}$ By extending the lifetime of a coating to five or even twenty years, and thus prolonging time between applications, costs of materials and labor may go 
down while aiding in more sustainable conservation practices. ${ }^{14}$ Coatings should be hydrophobic, have high thermal and photochemical stability and must resist the environment they are placed in (i.e. oxidation during outdoor exposure).

A fundamental study of coating performance during weathering was performed using complementary information from Fourier transform-infrared microspectroscopy ( $\mu$-FTIR) and EIS. Polymer resins comprised of primarily polyvinylidene fluoride, methyl methacrylate, and/or ethyl acrylate were formulated for clear-coating applications on outdoor weathered bronze or steel [See Scheme 5.1 in Section Error! Reference source not found.]. The best traditional coatings (such as solvent-based acrylic lacquers for bronze) and novel high-performance materials like PVDF/acrylic emulsions identified in the lab underwent extensive aging by both natural and accelerated weathering. In Chapter 5 we monitor the barrier properties and degradation of polymers weathered by accelerated QUV, outdoors in South Florida with salt spray, and outdoors in Portland, Oregon (details of the accelerated weathering cycles are shown in Figure 1.1).

(a)

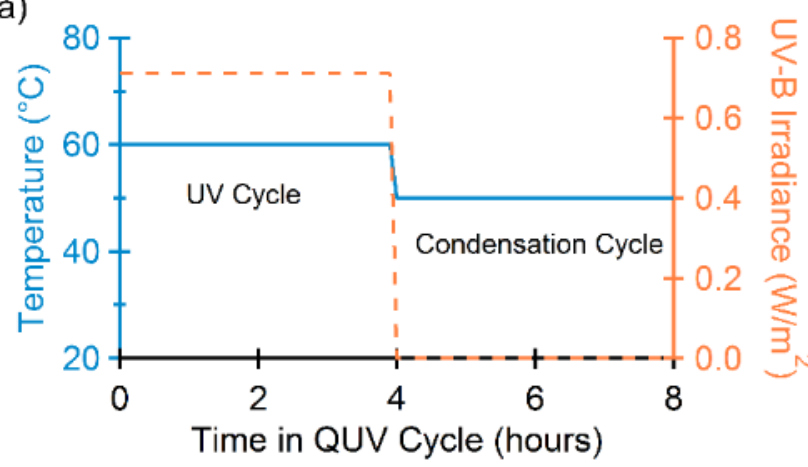

(b)

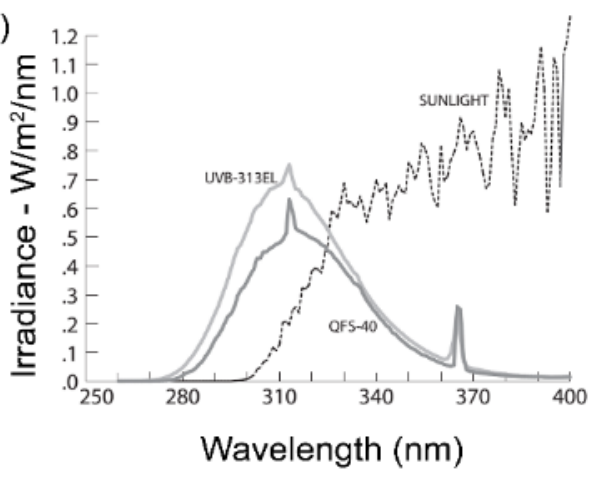

Figure 1.1 (a) Average conditions of accelerated weathering cycle in the QUV in and (b) spectral output of UV-B lamps which produce mostly short-wave UV radiation. 


\subsubsection{Coating lifetime}

This work aimed to produce realistic lifetime expectancies of high-performance coatings and diagnosed failure if and when it occurred. Using EIS, barrier properties were monitored to give values for minimum protective lifetimes, where every effort was made for non-destructive analysis so the same sample could be monitored during multiple time-points. Failure of the coating, and damage to the substrate beneath, have typically begun long before visual inspection reveals significant physical changes. Macroscopic defects such as cracking or delamination are frequently the result of chemical changes due to weathering. ${ }^{55}$ The goal of many scientists in recent years has been to identify analytical signatures that will track chemical changes and indicate coating failure long before degradation can be visually documented. Relevant theory from methods used in this research to monitor changes in the chemical structure and mechanical properties of polymeric coatings are explained in detail in the relevant chapters.

\subsubsection{Photochemical stability}

Corrosion prevention by coatings is largely dependent upon the chemistry and durability of the polymer to resist chemical change from UV irradiation. UV-polymer interactions induce micro-structural changes in the film that generally occur in three phases: 1) initiation, 2) propagation, and 3) termination..$^{5}$ Initiation occurs when energy in the ultra-violet wave range allows the polymer to produce free radicals via breakdown of a chemical bond, or photolysis. Free radicals can react with another 
species in the exposed environment or produce other free radicals (oxy- and peroxyfree radicals) in neighboring chains via propagation. Polymer chain scission or recombination of free radicals results in a carbonyl or alcohol containing substituent on the chain, terminating the propagation of the free radical. These general mechanisms for photooxidation are depicted in Figure 1.2, but there are many additional reactions that include recombination and cross-linking through chain-coupling as well as grafting of recently produced degradation products..$^{57}$

1) Initiation

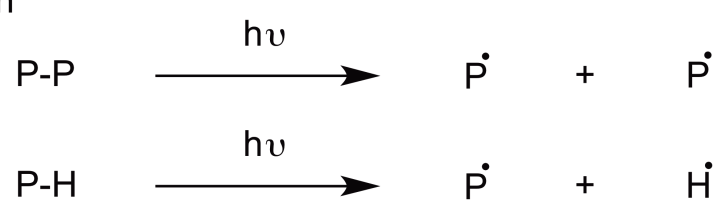

2) Propagation

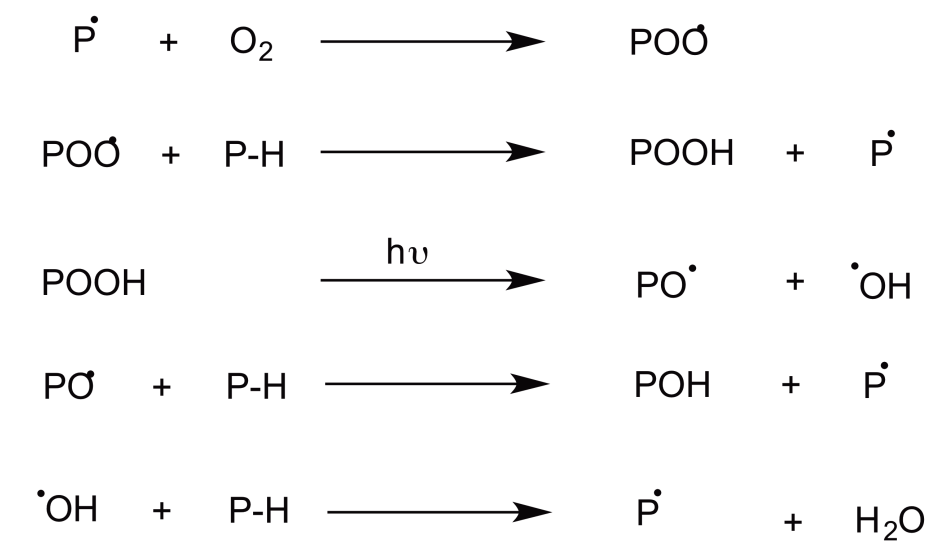

3) Termination

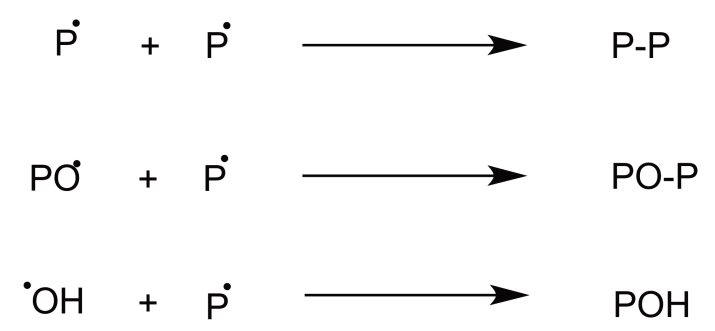

Figure 1.2 Example of general mechanisms for photooxidation. Steps include 1) Initiation 2) Propagation and 3) Termination. ${ }^{65} \mathrm{~A}$ number of more complicated pathways are not shown here, including recombination. 


\subsection{Polymer-clay nanocomposites as coatings for corrosion control}

The principle design of PVDF-clay nanocomposites (PNC) was guided by practicality and simplicity. The PNC itself is created by the exfoliation-adsorption method, where clay is exfoliated in a bulk solvent that the polymer is soluble or miscible with (here, we use water) and upon drying the polymer can interact with the particles by adsorption or intercalation, albeit the latter is less likely in a latex. ${ }^{37}$ Chemical functionalization of nano-clays with functional groups of compatible chemistry were expected to not only promote stability of individual platelets during early film formation, but increase the likelihood of inter-particle diffusion during coalescence.

\subsubsection{Nano-clays}

Clays are perhaps one of the most interesting particles rarely thought about in one's daily life, but they are everywhere in cosmetics, paints, toothpaste, cleaning agents, and colloidal suspensions. The physical parameters of Laponite nano-clay in particular made an ideal structure for this project, as the inorganic/thin-disk like material shown in Figure 1.3 allows for improvements in hydrophobicity and film hardness while retaining optical clarity. However, the charged interfaces and crystalline structure of many clays are difficult to control in solution and lead to a multitude of problems in coating systems and the application thereof. Langmuir famously observed long-range ordering of clay particles at a macroscopic scale, an outcome which no one has ever reproduced entirely. ${ }^{58}$ However, promise has been shown for the improved barrier properties of nanocomposites as the incorporation of silicates into PVC changed the refractive index 
of a coating, decreased gloss (\%reflection) and increased UV inhibition through suspected free radical particle trapping during.$^{59}$

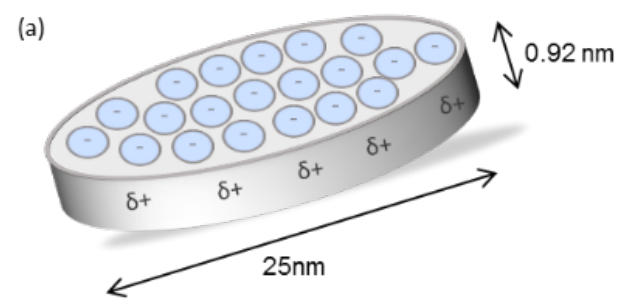

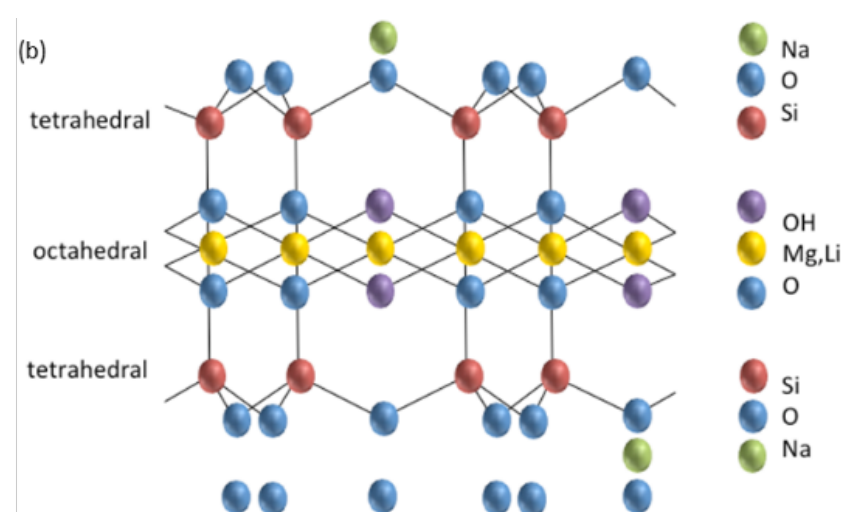

Figure 1.3 Synthetic nano-clay Laponite. (a) cystalline structure of 2:1 Laponite; (b) Single Laponite crystal, $25 \mathrm{~nm} \times 1 \mathrm{~nm}$. As reported by the manufacturer.

Laponite is a synthetic magnesium silicate in the smectite family whose closest natural counterpart is the mineral hectorite. It can be described by the chemical formula $\mathrm{Na}_{0.7}\left[\left(\mathrm{Si}_{8} \mathrm{Mg}_{5.5} \mathrm{Li}_{0.3}\right) \mathrm{O}_{20}(\mathrm{OH})_{4}\right]$ as a disc shaped 2:1 clay particle with repeating octahedrally coordinated magnesium/lithium oxide sandwiched between two tetrahedrally coordinated silica sheets. Laponite's unique properties come from its high aspect ratio (25), specific surface area $\left(350 \mathrm{~m}^{2} / \mathrm{g}\right)$, disc-like particle single crystals and charged surfaces. Sodium cations are found in abundance along the face of a platelet and in between discoid layers to counterbalance the overall negative structural charge of the smectites.

Dry silicates exist in bulk form as layered stacks randomly oriented to other clay sandwiches, as opposed to individual platelets. Their stacking is disrupted upon dispersion of the clay in an aqueous system by mechanically induced exfoliation (osmotic pressure) or intercalation (by some molecule or sheer stress). ${ }^{60}$ When Laponite 
nano-clay is fully wetted out in neutral water the interlayer sodium cations migrate out of the layer sheet to pull apart a $1 \mathrm{~nm}$ thick platelet by osmotic pressure. The accordionlike release of an individual platelet upsets the charge balance until there is a net negative surface (face) charge and an opposing partial positive charge from protonated metal oxides along the broken edges. Laponite is often used as a rheology modifier due to charged interfaces of the crystalline structure that allow for diffusion control in wet suspensions and can create a unique system of phases that range from sols to gels and eventually charged glasses. ${ }^{61}$

\subsubsection{Modification of silicates}

Laponite is a modifiable clay and as thus is capable of undergoing cation exchange on the face of the platelet (usually with charged sodium ions), gallery expansion, and covalent attachment through active silanol groups on the rim. The unique properties of Laponite nanocrystals create many possibilities for intended and unintended modification. Exfoliation is the process where platelets are separated from their natural stacking order in some fluid suspension. Due to charged rims and surfaces on Laponite, complete exfoliation of unmodified clay is possible in dilute solutions only.

Ion exchange is one type of surface adsorption where charged molecules of opposite sign are attracted to the high density of charge on the clay surface and will accumulate in the interlayer film of water to participate in the charge balance of the solid surface. A secondary step of displacement will occur if electrostatically favorable. For instance, higher valence atoms will displace lower valence atoms and when 
univalent, the smaller ion will displace the larger (e.g. $\mathrm{Li}^{+}$displaces $\mathrm{Mg}^{+}$). Mineral surface selectivity for certain ions can be overcome with high mass loading, making the exchange more favorable. At low $\mathrm{pH}$ the surface exhibits a positive charge and at higher $\mathrm{pH}$ with excess hydroxyls a net negative surface charge occurs.

Covalent modification constitutes the creation of a siloxane bond between a free silanol group on the clay's broken edge and a silane molecule with the chemical functional group of choice. For this work, target end groups of the silane esters consisted of a methyl methacrylate or a vinylidene fluoride monomer. The synthesis detailed in Chapter 6 was modified from other silane ester grafting procedures in airfree conditions. ${ }^{62-63}$ Inspired by recent advances in polymer bioconjugate design ${ }^{64}$ and the peptidizer commonly employed to stabilize clay platelets, ${ }^{65}$ a quaternary amine phosphoryl choline tetrahydrate was exchanged with sodium cations on the surface of Laponite. The large phosphate groups anchored to the clay surface were expected to aid in both exfoliation/dispersion of the clay discs to form an isotropic colloidal suspension before addition to the PVDF latex resin.

\subsubsection{Silicate nano-clays in PVDF latex films}

It was originally hypothesized that incorporation of nano-particles could scatter incoming UV radiation, absorb damaging free radicals in polymer chains, and establish a tortuous-path shown in Figure 1.4 for water molecules and ions to reach the substrate metal under a coating. The full potential of the possible long range ordering of clays is not well established due to the difficulty in controlling their surface charge which 
affects their orientational order ${ }^{66}$ Organically-modified nano-clay was added to the most promising coating formulations at loading levels sufficient to improve bulk properties of the polymer, but low enough so as not to interfere with film formation of the coating $(\sim 0.6 \%)$. Successful materials were well-characterized through changes from bulk powder, to isotropic suspensions, stabilization in the wet emulsion, and formation of a torturous pathway in the dry film for water molecules to reach the substrate (shown in Figure 1.5). Improved properties of the PNC coatings investigated in Chapter 6 included a reduction in mass transport by diffusion and water whitening of the films, both sensitivities only characteristic of waterborne coating systems.

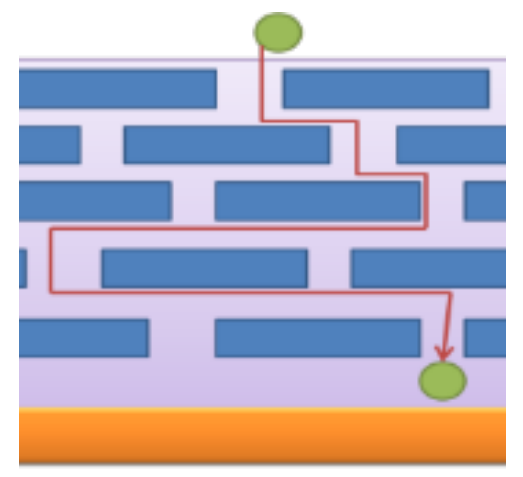

Figure 1.4 Torturous path for molecule to substrate.
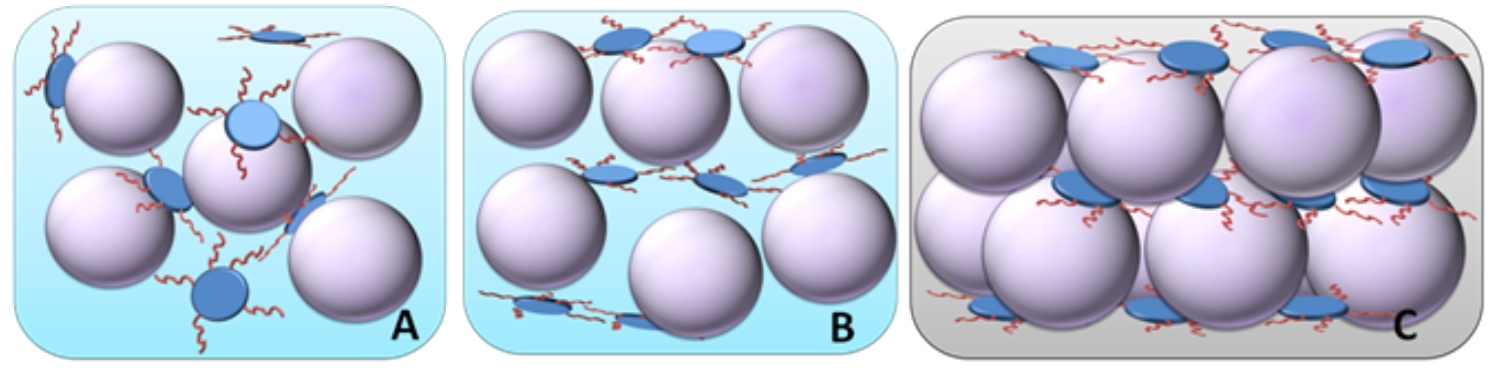

Figure 1.5 Cross section of modified clay (small disks) in polymer suspension (spheres): (a) Isotropic disorder in aqueous solution (b) Nematic ordering in wet emulsion (c) chemically compatible nano-clays in dry film. 


\subsection{Scope of this work}

The growth of materials science has been well timed to suit the unique and growing demands of conservation science. The work presented in this dissertation culminates in the development of a high-performance waterborne protective coating with excellent corrosion protection that could reduce the environmental impact of conservation treatments, as the most effective current options contain large quantities of harmful volatiles and require frequent re-application.

Given surprising resistance in the conservation community to moving away from the use of waxes as the favored protective coating, Chapter 2 outlines a basic investigation of the chemical and physical properties of common waxes used in protecting cultural heritage. Waxes were found to be fundamentally limited by their chemistry as poor barrier films due to high diffusion rates in non-close packed films and a susceptibility for oxidation from UV active groups substituted into the polymer.

Chapter 3 establishes baseline electrochemical performance of uncoated bronze panels pre-treated with a flash rust inhibitor as well as the electrochemical changes associated with film formation of both a common solvent-based and waterborne coating monitored by impedance spectroscopy. The equivalent circuit models used in practice to describe a number of polymers in different stages of their lifetime, including degraded films, ${ }^{67}$ suggests that partially formed films have similar barrier properties and electrochemical signatures as aged or weathered films. Water diffusion into coatings produces a swollen layer above an unaffected pristine layer in the coating, with the characteristic length scale and total quantity of the absorbed water affecting 
electrochemical performance. ${ }^{45,68}$ For this purpose in situ electrochemical spectroscopy used in Chapter 4 is ideally suited to differentiate active processes that change the barrier properties of the base and topcoat. ${ }^{69}$ Practical methods of post-application annealing were also explored in this chapter to improve initial coating barrier properties. Chapter 5 assigns protective lifetimes to traditional and novel treatments for conservations of cultural heritage metalworks. In addition the results of a blindstudy are reported where U.S. conservators applied and ranked gloss and workability of the coating materials used in this research.

The most significant hurdle for this project was controlling the nano-clays in aqueous suspensions and eventually dry films, the results of which are presented in Chapter 6. If the nano-clay platelets are not completely exfoliated, aggregation can occur. This results in macro-domains of gelled particles in the coating that can inhibit film formation and hinder coalescence into a complete polymer network. A torturous path was created in the waterborne PVDF coating with nano-clay additives, increasing resistance to both water and UV degradation. This chapter follows attempts at controlling the electrostatic properties of the nano-clay in water by cation-exchange, to dispersion in the wet emulsion, and ultimately the dry PVDF/acrylic film by covalent attachment. 


\section{On the protective nature of wax coatings for culturally significant outdoor metalworks: microstructural flaws, oxidative changes, and barrier properties}

Adapted with permission from: Swartz, N.A.; Clare, T.L., On the protective nature of wax coatings for culturally significant outdoor metalworks. J. Amer. Inst. Conserv., 2015, accepted.

Changes in chemical, microstructural and barrier properties of two commonly used and commercially available waxes - a microcrystalline wax (Renaissance wax) and a microcrystalline and carnauba blended wax (Butcher's Boston Polish Amber Paste Wax) -for the prevention of corrosion on outdoor metalwork were investigated. The waxes were applied to both plain and patinated substrates of bronze and steel, and properties of the films were measured before and after weathering. Accelerated weathering was performed by QUV-B illumination/condensation and outdoor weathering under standard South Florida exposures and in Portland, Oregon.

Comparison of $\mu$-FTIR and NMR spectra enabled characterization of chemical functional groups of these waxes as they weathered and showed relative increases in methylene character suggestive of cross-linking, appearance of terminal vinyl bands suggestive of chain scission and evidence of oxidative film damage. After annealing microstructural changes were observed by TF-XRD and showed a decrease in crystallinity which indicates a decrease in barrier properties. The barrier properties of the wax films after weathering were studied by EIS and equivalent electrical circuits provided insights into the physical state of the films. The EIS data showed that the permittivity of the weathered waxed panels increased, which was observed as a decrease in coating resistance and an increase in coating capacitance. Photographic documentation of the substrates showed extensive visible corrosion upon weathering. Our results demonstrate that waxed metal panels exposed outdoors do not have a period of stability when the protective qualities of the film do not change. This suggests that while waxes are commonly used to protect against corrosion, their barrier properties are affected by flaws in the microstructure and their susceptibility to chemical alteration during weathering. 
Results from a blind study with professional conservators as the participants are presented, to elucidate how they rank the importance of such factors as gloss and working lifetime when selecting protective coatings.

\subsection{Introduction}

Sculptures are coated to prevent corrosion (e.g. bronze disease and rust), which is a spontaneous and energetically favorable reaction. Since most materials used in the conservation of artwork are manufactured for other applications, little technical information is available for their performance lifetime on relevant substrates, such as bronze and steel. ${ }^{70}$ Although waxes are hydrophobic materials with low water vapor permeability and low gloss, properties seemingly ideal for protective coatings, their use as such on artistic and architectural metalwork may be problematic due to their short lifetimes, high VOC content, and resistance to removal. In a 2009 survey of 117 conservators, 54 conservators reported using waxes routinely on outdoor copper-based works (e.g. bronze and brass) and 27 reported having used wax routinely on steel. ${ }^{71}$ While waxes are widely used, conservators also recognize that wax films have finite lifetimes; the majority of respondents (45) reported that the expected lifetime of protective wax films on outdoor metalworks is 1 year or less and 32 respondents reported an expected lifetime of 2-5 years. Given those responses, it is probable that waxes are widely used for corrosion protection not because of their working lifetime, but rather for other reasons such as their aesthetics (because wax is a low-gloss coating, which is controllable by buffing) and because their working properties are wellknown. 
Chemically, waxes are mostly hydrocarbon chains, but their simple chemistry does not suggest homogenous microstructural properties. As waxes age, their chemical composition alters, as does their crystalline structure, grain size, bulk mechanical properties, solubility and workability. Technical studies aimed at elucidating the chemical and/or structural properties of waxes have focused on polyethylene, the principal molecular component of paraffin waxes. In 1956 and 1976 studies, infrared spectroscopy of the fingerprint spectral region was utilized to characterize chain-length and -branching. ${ }^{72}$ To gain further insight into the microstructures of waxes, XRD studies have correlated the microstructural relationships between grain size, melting temperature and barrier properties of the films. ${ }^{73}$ And further complicating our understanding of waxes, the proposed chemical degradation pathways and accompanying morphological changes determined vary widely in each study. ${ }^{74-75}$ Thus, replying upon specific conclusions from previous studies on the chemical, microstructural and changes in barrier properties of waxes that are commonly used for corrosion protection on artistic metalworks is not entirely possible.

Butcher's Boston Polish and Renaissance waxes were selected for this study because of their wide-spread use in the conservation field and commercial availability. ${ }^{7-77}$ Butcher's wax has a hard paste consistency and is composed of iso- and n-paraffinic microcrystalline wax and carnauba wax that includes esterified fatty diols that hydrolyze into fatty acids such as tetracosanoic acid $\left(\mathrm{C}_{24} \mathrm{H}_{48} \mathrm{O}_{2}\right)$ and fatty alcohols such as triacontanol $\left(\mathrm{C}_{30} \mathrm{H}_{60} \mathrm{O}_{2}\right){ }^{78}$ Renaissance wax is a refined mixture of high molecular weight iso-paraffins blended with mineral spirits and has a soft paste 
consistency. Because Butcher's wax has a higher acid content, it is predicted to be more reactive and have a shorter lifetime than Renaissance wax. Previous studies of waxes by conservators or conservation scientists suggest that their weaknesses are known: having poor barrier properties, ${ }^{79}$ they may photo-degrade ${ }^{76}$ and their films can be difficult to remove completely. ${ }^{77,80-81}$ Electrochemical investigations of wax/patina combinations have indicated that artificial patinas alone are not protective in outdoor environments but can be improved with a waxy top coat. ${ }^{82}$ While sometimes providing a metric for failure, scant previous data exists that correlates microstructure and chemical changes with failure in the barrier properties of wax films. The aim of this work is to gain a more thorough understanding of the protective quality of wax films using multiple analytical characterization methods. Additionally, the authors aim to provide useful points of comparison for future evaluations of protective coatings that may offer better corrosion protection and have significantly longer working lifetimes than these waxes.

\subsection{Background}

\subsubsection{Structure of waxes}

Waxes are principally composed of high molecular weight alkanes, typically between 20-50 carbon atoms in each chain. Most waxes contain microstructural crystalline regions, or platelets, that along with their hydrophobic chemistry, make them resistant to ion and vapor transport. ${ }^{73}$ The crystalline phases are composed predominately of long-chain aliphatic molecules of varying length organized by chain-chain and tail-tail 
stacking and are surrounded by disordered chains to create amorphous regions, ${ }^{83}$ as depicted in Figure 2.1. Branched alkanes are produced during the Fischer-Tropsch synthesis of waxes (including Renaissance); and it has been proposed that a driving

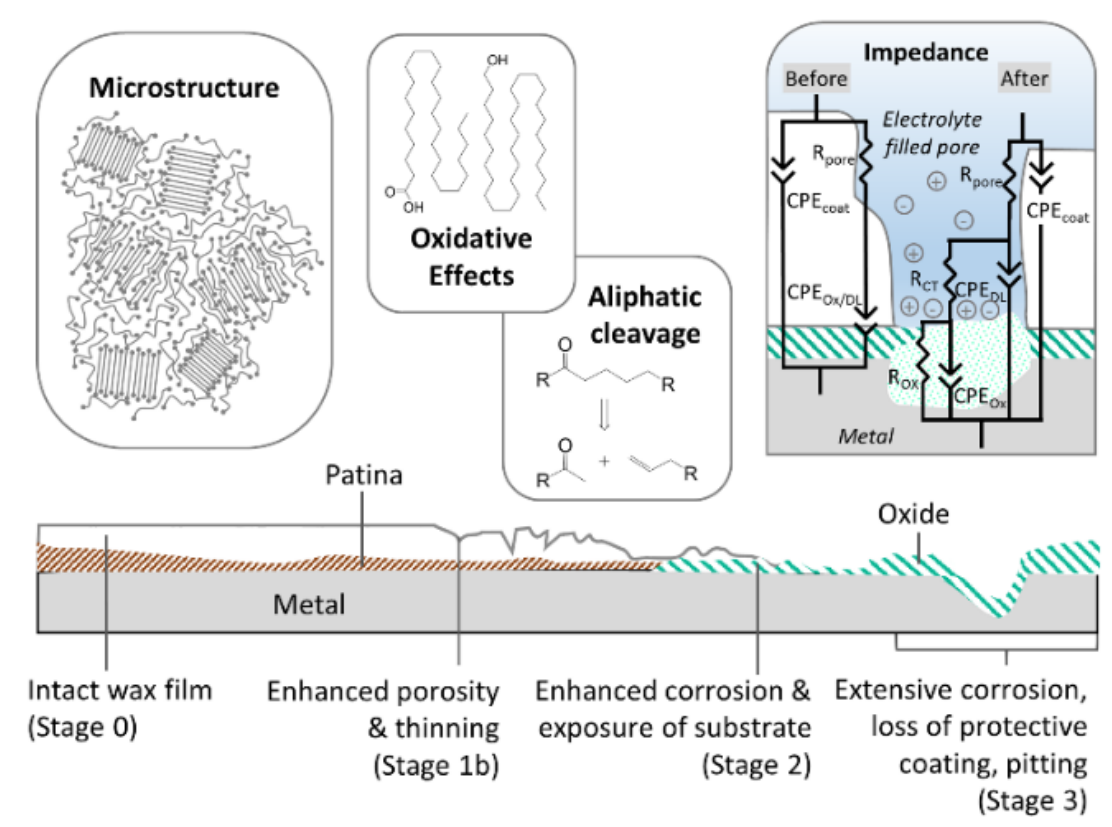

Figure 2.1 Schematic of a waxed metal substrate at various stages of deterioration. Insets detail the structural, chemical and barrier properties of the system.

force for change in film crystallinity results from the exclusion of branches from the majority linear alkanes' crystalline phases..$^{72}$ It is possible (and often suggested) that annealing may heal defects in some organic coatings, ${ }^{84}$ but in the case of microcrystalline wax, thermal treatment can increase heterogeneity of the fresh waxed film. Evidence of structural alteration in heated polyethylene-based materials can be observed using XRD and DSC, where thermally-induced expansion of the unit cell has been noted in both naturally derived and synthetically produced waxes. ${ }^{74}$ As in any organic coating, heat may also promote early onset of oxidation of the film as indicated 
by several IR investigations that showed the presence of oxygen-containing degradation products. ${ }^{72,85-87}$ Because conservators of outdoor metalwork often use heat to anneal wax coatings to drive off solvent and harden the films, the effect of annealing on the microstructure of wax films was examined to better understand the relationship between structure, barrier properties and performance lifetime of those films.

\subsubsection{Impedance spectroscopy of organic coatings}

As coatings age, defects such as pores absorb electrolyte which can dramatically affect the impedance of the coated system. In Electrochemical Impedance Spectroscopy (EIS), a coated metal is immersed in electrolyte and a small AC potential is applied to the metal working electrode. The opposition (impedance) of current flow through the protective coating (wax) is monitored at the opposing counter electrode (an immersed graphite rod) over a range of frequencies. This setup allows determination of a coating's impedance $|Z|$, which is related to the coating's barrier properties, and can be understood as its ability to slow mass transport from the bulk electrolyte to the metal surface (e.g. impede water, ions, charge-transfer, and other processes associated with corrosion). Thus an ideal protective coating acts like a capacitor and as the coating degrades additional electrical circuit elements can be observed by EIS - such as the pores (which act like resistors and are referred to as $\mathrm{R}_{\text {pore }}$ ). Corrosion occurring at the coating-metal interface is depicted in Figure 2.1 and results in the formation of an additional capacitive element, the diffuse double-layer $-C_{d l}$, and a resistive charge- 
transfer process - $R_{c t,}$. Coated panels having lower total capacitances hold less charge/electrolyte and should have better protective properties than those having higher capacitances. Likewise, films having lower resistances allow the passage of ions, indicating poorer barrier properties than those having higher resistances.

EIS is a technique that is highly sensitive to defects in coatings. For example, a defective area that is only $0.005 \%$ of the coated area may have a capacitance of $20 \mu \mathrm{F}$, which would be equal to the entirety of a typical coating's capacitance of $1 \mathrm{nF} .^{88}$ As a point of comparison, solvent-based or waterborne acrylic films have capacitance values ranging from $0.1 \mathrm{nF}$ to $10 \mathrm{nF}$ and have film resistances ranging from $10 \mathrm{M} \Omega$ to $1 \mathrm{G} \Omega .{ }^{37}$ The presence of capacitive elements in EIS spectra are observed in the magnitude (|Z|) versus frequency plots as sloping lines, while resistors are observed by plateaus. In the phase versus frequency plots, capacitors are "out-of-phase" with the applied AC potential and approach $-90^{\circ}$ while resistors are "in-phase" and approach $0^{\circ}$. In constructing equivalent electrical circuits (EECs), the number of circuit elements correspond to the same number of spectral regions dominated by capacitors or resistors. Coated metal panels act like non-ideal capacitors so constant phase elements (CPEs) are commonly substituted to account for frequency-dependent anomalies. ${ }^{20,} 89-90$

Changes in the barrier properties of weathered wax coated metal substrates were studied using EIS. By producing EECs and monitoring the values of individual circuit elements over time, the onset of coating failure as well as complete failure can be determined. After weathering either additional circuit elements or changes in the values of circuit elements are observed; and as such, are indicative of coating 
degradation with formation of additional or larger pathways to the active metal surface. ${ }^{88,90-91}$ The total impedance at low frequencies, $|\mathrm{Z}|_{0.1 \mathrm{~Hz}}$ is a commonly used metric to compare coating performance. ${ }^{11,20-21,53,84,92-94}$

\subsection{Methods}

\subsubsection{Details of the Blind Study}

In 2012 the authors sent kits to 13 professional conservators from the USA who agreed to participate in this blind study to qualitatively evaluate protective coatings used for artistic metalwork. The kits each contained six different coatings, including Renaissance wax, in tins labeled with a code number (not a name) along with application instructions, patinated bronze and steel substrates numbered for their intended coating(s), cloths, brushes and gloves. Application instructions stated that the panels were not to be annealed or heated in any manner. After applying the coatings, each participant anonymously completed an online exit survey on the glossiness of the coatings. The panels were then returned to the lab for further analysis that included gloss, thickness and impedance measurements. Only the results for wax coated bronze and steel panels are reported here.

\subsubsection{Sample Preparation}

Bronze substrates ( $90 \% \mathrm{Cu}, 10 \% \mathrm{Sn}$, TB Hagstoz \& Son Inc.) and steel substrates ( $99.5 \%$ $\mathrm{Fe}, 0.10 \% \mathrm{C}, 0.35 \% \mathrm{Mn}, 0.04 \% \mathrm{P}, 0.05 \% \mathrm{~S}, \mathrm{Q}-\mathrm{Labs}$, Inc.) were prepared as described below. Coatings in this study were Butcher's Boston Polish Amber Paste Wax (carnauba and microcrystalline waxes blended with mineral spirits) or Renaissance Wax 
(Cosmolloid 80 and BASF A waxes blended with mineral spirits). Thin layers of wax pastes were applied to a warmed bronze or steel panel with a cotton cloth in alternating direction and buffing of dried films between applications to produce a film thickness of $20 \pm 2 \mu \mathrm{m}$. Thickness and gloss measurements were the average of five trials with a Gardco $\mu$-Tri-Gloss/thickness meter calibrated on the substrate under study and \% Reflectance at ${ }^{\circ} 85$ with a black glass reference. Images acquired with a stereoscopic light microscope (Leica MZ6) or Nikon D40 DSLR.

Preparation of substrates before waxing. Bronze and steel panels were degreased by soaking in xylene followed by isopropanol, each for 10 minutes and dried with cotton after each soak. Panels were sanded with increasing grit from 400 to 600 to 1000 grit wet/dry SiC paper in alternating directions until an even luster was obtained, rinsed with DI water between grits and a final rinse with isopropanol. For plain substrates wax was directly applied to the bare metal. For patinated substrates freshly sanded and degreased panels were immersed in a $20 \%$ by volume patination solution of Antique Black (Birchwood Casey, Inc) for four minutes and then rinsed with water and dried with compressed air. The process was repeated three times to ensure a complete patina. Substrates were rinsed with ethanol followed by acetone prior to coating to remove any residual "active" species. The patination solution was recommended for use by Mr. Andrew Lins, Chair of Conservation at the Philadelphia Museum of Art, and is sold by Birchwood Casey with the following composition in water $\left(\% \mathrm{w} / \mathrm{w}_{2} \mathrm{O}\right)$ : selenous acid 
(4\%), phosphoric acid (8\%), cupric sulfate (4\%), ammonium molybdate ( $4 \%)$, and zinc sulfate (4\%).

\subsubsection{Weathering Exposure}

All panels to be weathered were first annealed four hours at $60^{\circ} \mathrm{C}$ in an oven prior to exposure to drive out residual solvent from the films, enabling a more accurate comparison of chemical species present before and after weathering. Substrates were exposed for $250 \mathrm{~h}$ time periods in a Q-Lab QUV chamber and weathered according to ASTM G154 Cycle 2: $4 \mathrm{~h} \mathrm{UV-B}\left(60^{\circ} \mathrm{C}, \mathrm{E}_{\mathrm{e}}=0.73\right)$ and $4 \mathrm{~h}$ condensation $\left(50^{\circ} \mathrm{C}\right.$, distilled water). Up to 36 months of south-facing, $45^{\circ}$ outdoor exposure was performed in Portland, Oregon (PDX) with periodic cleaning with distilled water. PDX panels were returned to the lab for analysis at 3, 9, 18, 36 months for bronze and 6 and 18 months for steel. Salt accelerated exposure was performed by Q-Labs in Homestead, Florida (FL) for up 18 months according to ASTM D6675. Panels were returned to the lab for analysis at 9 and 18 months for bronze and 6 months for steel.

\subsubsection{Thin-film X-ray diffraction}

Thin film X-ray studies were acquired in-situ on the weathered panels with a Rigaku Ultima IV Multipurpose X-ray diffractometer using a Cu-K $\alpha$ radiation source $(\lambda=1.542 \AA)$ in parallel beam geometry and step size $=0.025^{\circ}$ for $5 \mathrm{sec}$ with a 2theta axis. Panels were aligned as-is by height (Z-axis) and center (omega) for acquisition of a $20 \mathrm{~mm}^{2}$ sample area. Diffraction spectra were processed with PDXL software and library spectra from the International Center for Diffraction Database (ICDD) was used for 
confirmation of products. An orthorhombic space group Pnam (n-paraffin, ICDD: oo040-1995) was used to optimize the unit cell by the Wiliamson-Hall method.

\subsubsection{Infrared Analysis}

A ThermoScientific iS 10 infrared spectrometer with a Nicolet Continupm FT-IR microscope and $250 \mu \mathrm{m} \mathrm{MCT} \mathrm{detector} \mathrm{was} \mathrm{used} \mathrm{to} \mathrm{acquire} \mathrm{IR} \mathrm{spectra} \mathrm{from} \mathrm{4000-650}$ $\mathrm{cm}^{-1}$ with $4 \mathrm{~cm}^{-1}$ resolution with Omnic software. Data was transformed using an N-B strong apodization function and Mertz phase correction. Micro-sampled cross sections were flattened before spectral acquisition in transmission mode for 512 scans on a diamond slide. All infrared spectra were normalized by peak area to the isolated symmetric methyl vibration at $1377 \mathrm{~cm}^{-1}$.

\subsubsection{NMR Analysis}

Approximately $5 \mathrm{mg}$ of each wax sample was dissolved in benzene- $\mathrm{d}_{6}$ with $1.0 \% \mathrm{TMS}$ as a shift reference. ${ }^{1} \mathrm{H}$ Proton scans were acquired with a Bruker $600 \mathrm{MHz} \mathrm{NMR}$ spectrometer over 64 scans with a TXI probe at $25^{\circ} \mathrm{C}$. Coupling and peak assignments were verified with $2 \mathrm{D}$ COSY and HSOC spectra acquired with $n=4$ scans for ${ }^{1} \mathrm{H}$ and $n=64$ scans for ${ }^{13} \mathrm{C}$.

\subsubsection{Electrochemical spectroscopy}

Potentostatic sweeps of a $14.6 \mathrm{~cm}^{2}$ working electrode area were acquired from $0.1-10^{6}$ $\mathrm{Hz}$ with $\mathrm{AC}$ voltage $20 \mathrm{mV} \mathrm{rms}$, and $0.0 \mathrm{VDC}$ vs. open circuit potential $-200 \pm 80 \mathrm{mV}$ with Gamry Reference 600 Potentiostat and Gamry Framework software. To ensure reproducibility of spectra: the coating was equilibrated for 30 min before acquisition 
where the counter and reference electrodes were kept at a fixed distance from the metal surface, in the same volume of $3 \% \mathrm{NaCl}$ (w/w in deionized $\mathrm{H}_{2} \mathrm{O}$ ) electrolytic solution with a calculated resistivity of $15.4 \Omega \cdot \mathrm{cm}$. Spectra were interpreted and modeled against EECs with Scribner Associates ZView. Errors in fit EEC models to the data were calculated as the sum of residual error where the fit differed from the experimental data. Average instrument error ranged from $10 \%$ at $0.1 \mathrm{~Hz}$ to less than $0.5 \%$ at $1 \mathrm{MHz}$. Capacitance values were calculated at their maximum angular frequency $(\omega)$ according to $C=C P E \times \omega^{\alpha-1} .95-96$

\subsection{Blind Study}

The results from blind study participants on the importance of protective lifetime and of a coating's gloss level are shown in Figure 2.2a-b. Based on conservators' responses, coatings having long working lifetimes are slightly more likely to be used than those that do not have long working lifetimes and confirmed gloss is a very important factor among many variables in selecting a coating. Study participants were then asked to rate the level of gloss of each panel. The responses, which were the same for either substrate, show in Figure $2 \mathrm{c}$ that $75 \%$ of conservators felt the unbuffed panels had insufficiently low levels of gloss. Upon return to our laboratory, the percent reflectance of each waxed panel was measured at $85^{\circ}$ with an average of $31.7 \pm 11.0 \%$ for bronze and $20.7 \pm 12.7 \%$ for steel. In the survey's comments box, several participants reported that had the application instructions permitted buffing, the panels likely would have acceptable gloss levels. Buffing was not permitted according to the application 
instructions to facilitate cross-comparison of the samples. Buffing waxed panels can involve the use of heat to drive off solvent which hardens the film, but heating may also have the undesirable side effect of oxidizing the films. For comparison, buffed panels in our weathering study had initial gloss values between $24-53 \%$ reflectance (gloss data for each panel is provided in Appendix A).

(a) Likelihood of choosing "Long Lifetime"

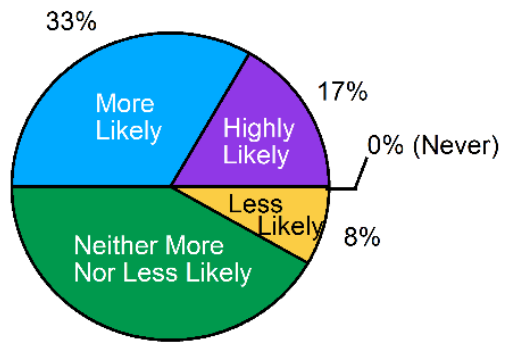

$42 \%$ (b) Importance of Gloss

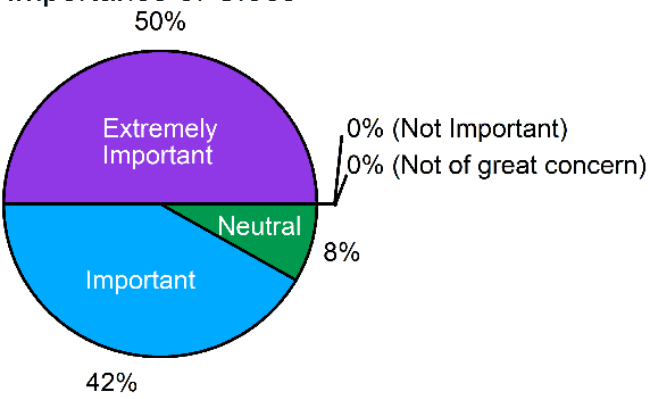

(c) Gloss rating of substrates
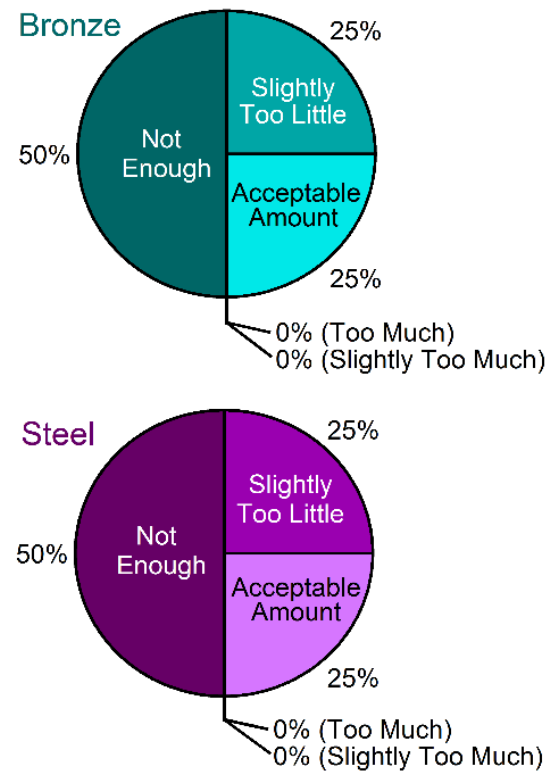

(d) EIS of substrates
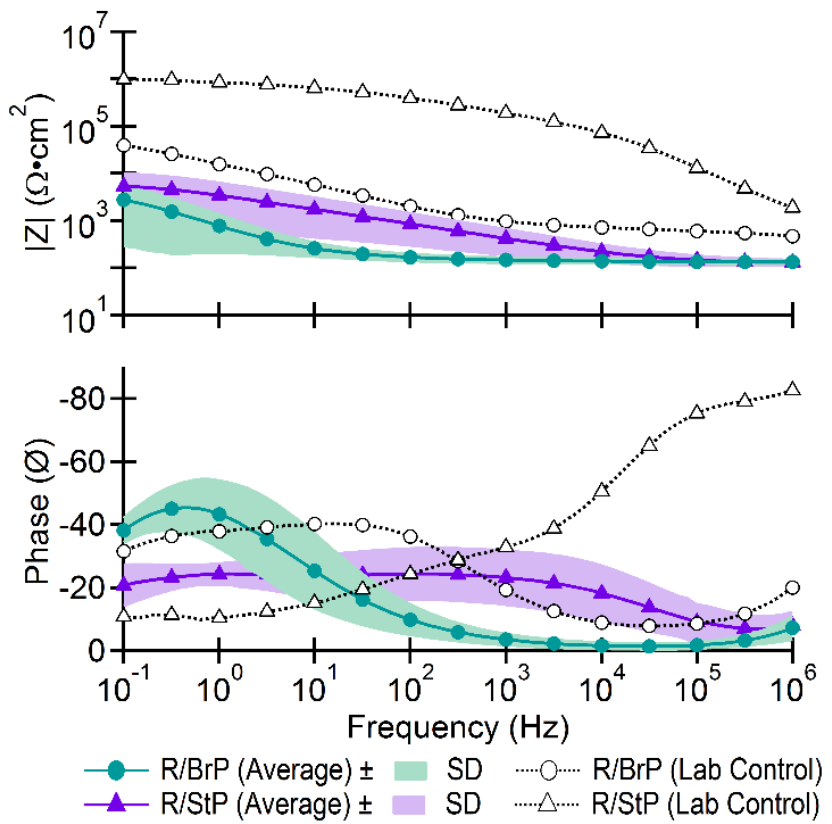

Figure 2.2 Averaged EIS Bode plots of wax coated bronze and steel samples by blind study participants (solid points) compared with laboratory control samples (open points). The error bars denote one standard deviation from the averaged data. 
An impedance spectrum of each panel that had been coated by a study participant was acquired (by the authors) and the spectrum from each panel was averaged; the average EIS spectra are displayed as Bode plots in Figure 2.2d, with one standard deviation of the EIS signal indicated by the shaded regions. For comparison, the authors prepared waxed substrates following the same application procedure provided to the participants, and the EIS spectra acquired from those panels are also shown in black with open markers (Fig. 2.2d). Significant sample to sample variation was observed in samples prepared by conservators, suggesting that precise details of the application method affect the measured impedance (and underscoring the sensitivity of this analytical method). The study participants' averaged spectra showed that a $200 \Omega \cdot \mathrm{cm}^{2}$ resistor corresponding to the electrolyte solution resistance dominated a large range of the measured frequencies (from $1 \mathrm{MHz}$ to about $10 \mathrm{~Hz}$ on both bronze and steel) which suggested poor coverage (since a resistor corresponding to a porous coating did not dominate the spectrum). The fluctuation in the phase angle at lower frequencies showed that diffusion of ions through the films was observed in the spectrum and active corrosion was occurring at the metal-wax interface in nearly all samples. Of the 26 returned samples ( 13 each for bronze and steel), none of the panels' impedance was greater than $10 \mathrm{k} \Omega \cdot \mathrm{cm}^{2}$ at $0.1 \mathrm{~Hz}$. For comparison, bronze panels coated with wax in the lab showed that from $1 \mathrm{MHz}$ to $100 \mathrm{~Hz}$ the impedance was dominated by a (larger) $1 \mathrm{k} \Omega$ resistor, which was the pore resistance of the coating. The waxed steel panel (prepared by the lab) was dominated by a capacitor and a $100 \mathrm{k} \Omega$ resistor, and these suggested that the lab's panel had better coverage than did those 
prepared by conservators. The differences between the lab's and conservators' panels may be due to marring during shipping (though visible marring was not observed) and/or by the advantage of having available equipment to assess the protective quality of coatings (EIS) and thereby improve upon application technique during the course of the lab's study of coatings.

\subsection{Effects of Annealing wax films}

\subsubsection{Changes in microstructure}

To better understand the effects of crystalline microstructures on film hardness (e.g. crystalline structure, size, percent crystallinity) and how annealing affects those metrics in wax films, TF-XRD spectra were acquired. Shorter chain alkanes have slightly lower melting points than longer chain alkanes when the molecules are chemically identical in all other aspects. It has been reported that thermal treatment can induce the smaller molecules to migrate from a crystalline lamellar structure into a mobile-amorphous phase between crystallites ${ }^{97}$ Another measure of microstructural changes comes from determination of percent crystallinity, where recent studies show that heat treatment can decrease the extent of crystallinity in paraffinic waxes. ${ }^{73,75,98}$ Experimentally, those determinations are made by calculating the ratio of wax in the hexagonal crystalline lattice [110], which is softer, to the orthorhombic lattice [020], which is harder to give a measure of the relative softness of the film (with larger ratios corresponding to softer films). According to ASTM D5357 percent crystallinity can be quantified using the crystalline peak area to total peak area (crystalline + amorphous halo, at $18.352 \theta$ for 
Renaissance wax and $16.842 \theta$ for Butcher's wax). To determine changes in crystallite size, the data were modeled using a standard polyethylene orthorhombic space group of the methylene unit cell, shown in Figure 2-3a, 2-3c, and 2-3d. We hypothesized that annealing may heal defects in wax films by: (1) molecular rotation at sites of chain kinking, (2) migration of lower molecular weight/lower melting temperature species, such as oils, or (3) exclusion of some branched species from some phases of the material. It was expected that those changes would be observed experimentally by an increase of average crystallite size and percent crystallinity.

a)

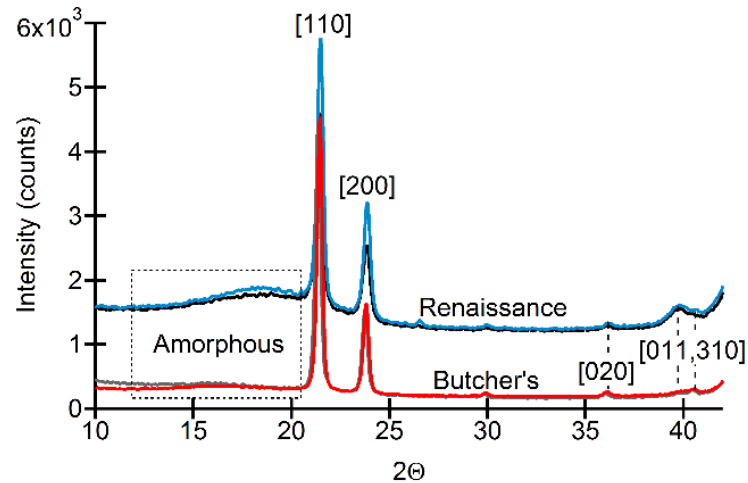

b)

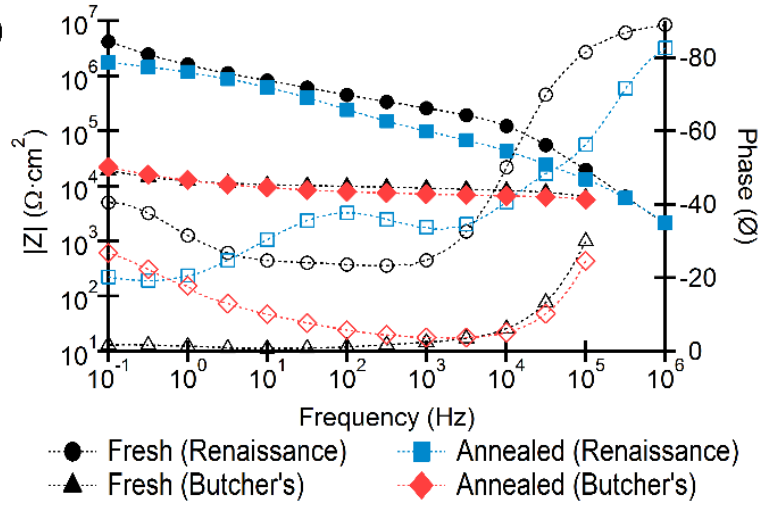

c)

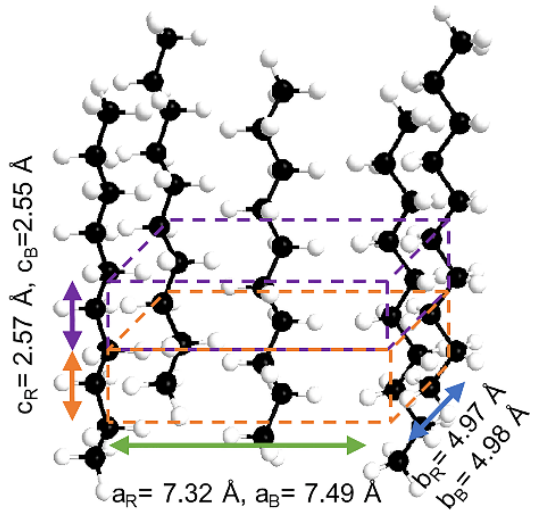

d)

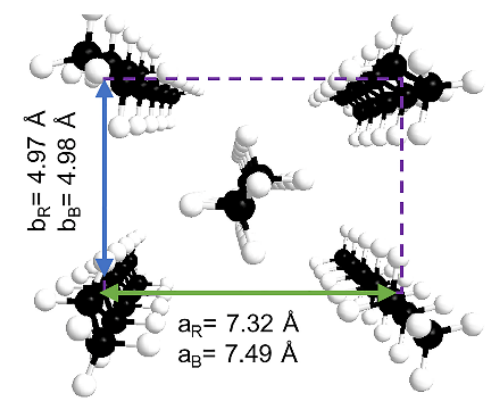

Figure 2.3 Spectral changes upon annealing of waxes. a) TF-XRD spectra of Renaissance and Butcher's wax, and b) EIS Bode plot where solid points are impedance magnitude on the left axis and open points are phase on the right axis. An orthorhombic space group Pnam was used to optimize the unit cell. Fit dimensions before [and after] annealing are shown with c) side view and d) top-down of the unit cell used to model Renaissance wax: $a=7.32 \AA[7.33 \AA], b=4.97 \AA[4.97 \AA]$, and $c=2.57 \AA$ [2.58 $\mathrm{A}$ ] and Butcher's wax: $a=7.49 \AA[8.47 \AA], b=4.98 \AA[4.96 \AA]$, and $c=2.55 \AA[2.56 \AA]$. 
Under the $60^{\circ} \mathrm{C}$ annealing conditions in this study, the films were softened but not completely melted. DSC thermograms were measured for both unweathered waxes to determine the melting point of each wax and were $61.6^{\circ} \mathrm{C}$ for Butcher's Wax and $80.3^{\circ} \mathrm{C}$ for Renaissance Wax (DSC traces are included in Appendix A). The hex/ortho ratio $\left(\mathrm{I}_{110} / \mathrm{I}_{020}\right)$ for wax films is shown in Table 2.1. From these data, it is apparent that a slightly greater fraction of the wax exists in the softer hexagonal lattice before annealing for both types of waxes, suggesting that one of the effects of annealing is to harden the film by conversion to the orthorhombic structure. The data show that the fraction of wax occupying the hexagonal structure is greater in Butcher's than in Renaissance wax, indicating Butcher's wax films should be softer than Renaissance wax films (consistent with the DSC data); and this held true both before and after annealing.

Table 2.1. TF-XRD and EIS of waxes before and after annealing

\begin{tabular}{lcccc}
\hline Wax/treatment & $\begin{array}{c}\text { Crystallite size } \\
(\AA)\end{array}$ & $\begin{array}{c}\mathrm{I}_{110} \\
\mathrm{I}_{020}\end{array}$ & $\begin{array}{c}\text { Rpore } \\
\left(\mathrm{k} \Omega \cdot \mathrm{cm}^{2}\right)\end{array}$ & $\begin{array}{c}\text { Ccoat } \\
\left(\mathrm{nF} \cdot \mathrm{cm}^{-2}\right)\end{array}$ \\
\hline Renaissance/before & 17.3 & 2.31 & 189 & 0.0896 \\
Renaissance/annealed & 29.7 & 2.03 & 38 & 0.131 \\
Butchers/before & 24.3 & 2.97 & 7.6 & 0.162 \\
Butchers/annealed & 52.2 & 2.81 & 6.8 & 0.185 \\
\hline I = Intensity and FWHM = peak width at half maximum intensity of wax \\
peaks measured at [110] = 21.5 $2 \theta$ or [020] = 23.9 $2 \theta$
\end{tabular}

The TF-XRD data show that the percent crystallinity decreased in Renaissance wax from $78.1 \%$ to $66.7 \%$ after annealing and increased in Butcher's wax: $69.5 \%$ to 79.6\%. Average crystallite sizes are listed in Table 2.1 and this value increased upon annealing for both waxes. These data also show that the two waxes differed in their 
average crystallite sizes: Renaissance wax had smaller crystallite sizes than Butcher's wax both before and after annealing.

From these data we conclude that annealing these wax films has the effects of hardening the waxes by producing more orthorhombic crystallites and migration of mis-folded or lower molecular weight material from the crystalline grains to the amorphous interstitial spaces. Interestingly, opposing effects on percent crystallinity upon annealing were observed in the two different waxes. It is expected that crystallite grain size, structure and percent crystallinity play roles in the barrier properties of films, which was investigated using EIS.

\subsubsection{Changes in barrier properties upon annealing}

EIS spectra of Renaissance and Butcher's waxed bronze panels before and after annealing were fit to the EEC labeled 'before' in Figure 2.1 and the spectra are overlaid in Figure 2.3b. Upon heat treatment the pore resistance $\left(R_{\text {pore }}\right)$ decreased from 189 to $38 \mathrm{k} \Omega \cdot \mathrm{cm}^{2}$ for Renaissance wax but showed negligible changes for Butchers wax (from 7.6 to $6.8 \mathrm{k} \Omega \cdot \mathrm{cm}^{2}$ ). The decrease in pore resistance in Renaissance wax films implied that the barrier properties of that wax film decreased after annealing, whereas those of Butcher's waxed films did not change appreciably. This difference in behavior of the pore resistance values may be related to the changes in percent crystallinity observed in the XRD data, with a greater percentage of the molecules occupying the more loosely packed amorphous phase after annealing in Renaissance wax compared to Butcher's wax. Additionally the capacitance of both films increased upon annealing, Butcher's 
wax by a factor of 1.1 and Renaissance wax by a factor of 1.5 , although $C_{\text {coat }}$ was consistently lower for the latter. Because the crystallite grains are smaller for Renaissance wax, the total area of the inter-granular regions is smaller, making the capacitance smaller. Comparatively, Butcher's wax has larger inter-granular regions (due to having larger crystallites) and so its capacitance is greater than for Renaissance. Hence an increase in amorphous, branched and/or lower molecular weight material in the inter-granular regions led to a decrease in coating resistance (faster ion transport to the metal) and an increase in coating capacitance (more volume to store ions in the coating matrix). Therefore, while annealing was observed to decrease the overall barrier properties of both wax coatings, it was more detrimental to the Renaissance wax films.

\subsection{Weathering}

\subsubsection{Changes in Molecular Structure}

mFTIR and ${ }^{1} \mathrm{H}$ NMR spectra of both waxes were collected before and after various types of weathering on plain bronze and steel substrates (Figs. 2.4-2.5). Plain substrates were selected for these analyses, rather than patinated, since they lack possible spectral interferences from a patina. Both NMR and mFTIR techniques were used because each provides slightly different information: FTIR is less sensitive to certain functional groups (particularly non-polar groups like alkanes) than NMR, while NMR can only measure soluble species (such as shorter chain alkanes, not extensively cross-linked materials). We characterized both fresh and annealed waxes as control samples before 


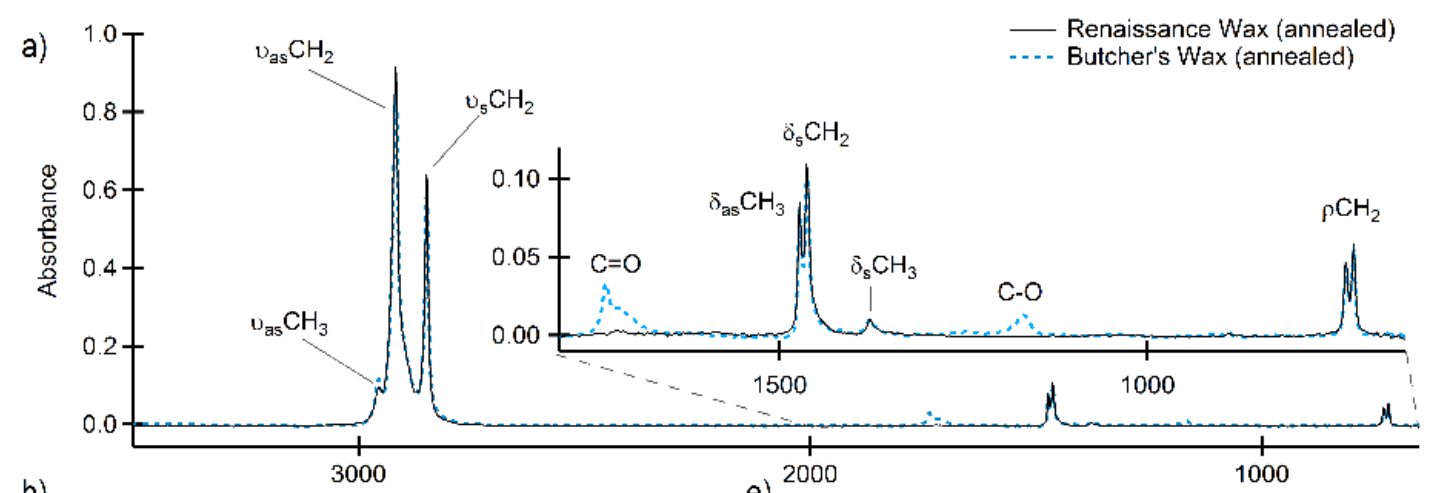

b)

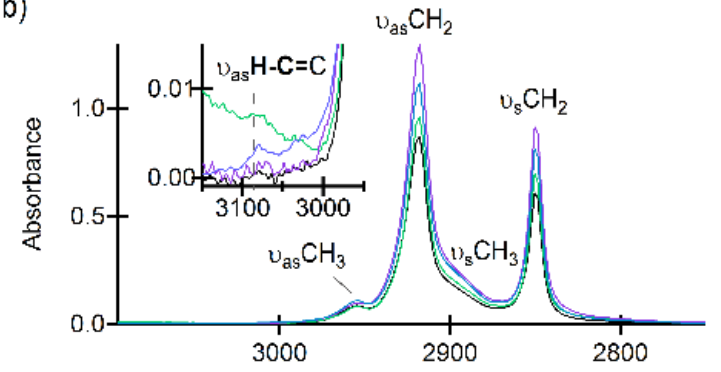

c)
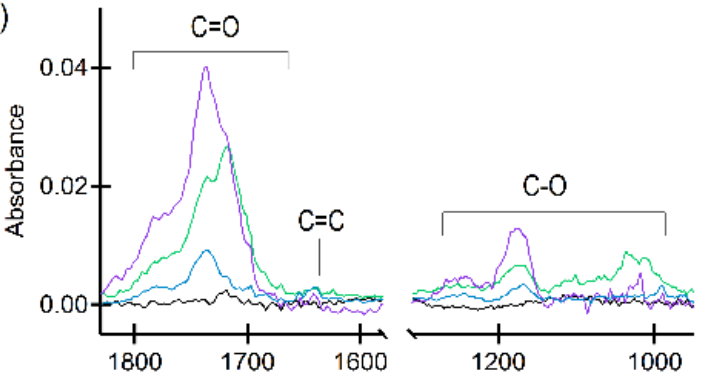

d)

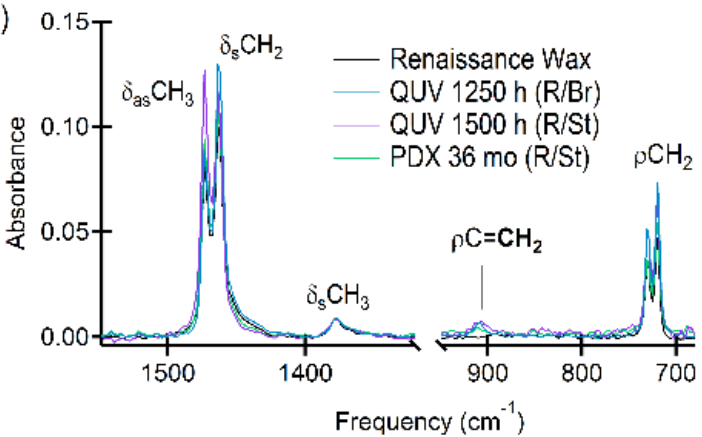

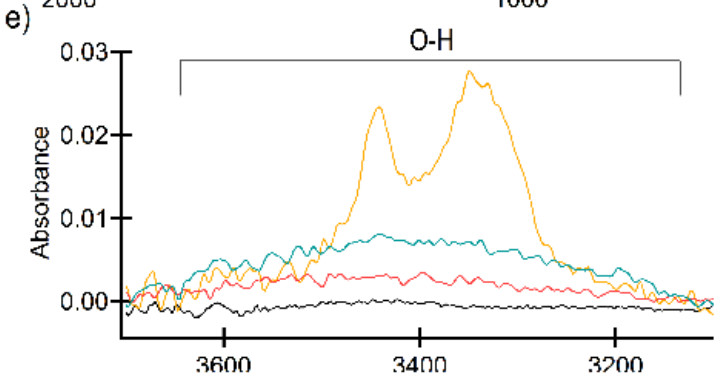

f)
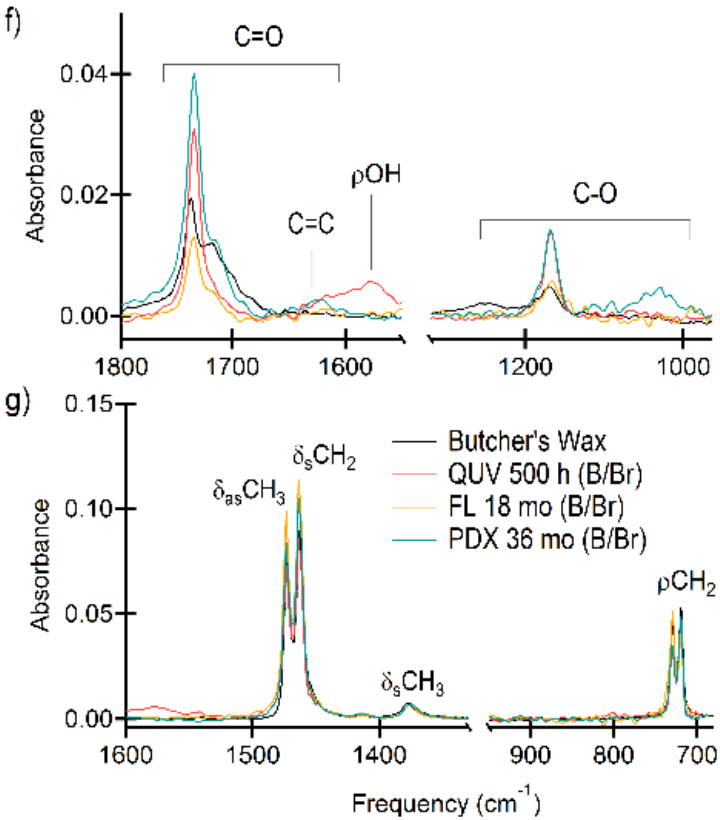

Figure 2.4 Infrared spectra from Renaissance and Butcher's waxed panels before and after weathering. a) Annealed Renaissance wax (black) and Butcher's wax (dotted blue) before weathering. b-d) Renaissance and e-g) Butcher's wax after weathering by accelerated OUV-B and outdoors in S. Florida and Portland, OR, where R|Br|QUV (blue), R|St|QUV (purple), R $|S t| P D X$ (green), B|Br|QUV (red), B|Br|FL (yellow) and $\mathrm{B}|\mathrm{Br}| \mathrm{PDX}$ (teal). Spectral regions were expanded for clarity to show b) aliphatic $\left(\mathrm{C}-\mathrm{H}_{3}\right.$ and $\left.\mathrm{C}-\mathrm{H}_{2}\right)$ stretching vibrations; $\mathrm{C}$ ) carbonyl $(\mathrm{C}=\mathrm{O})$, vinyl $(\mathrm{C}=\mathrm{C})$, and ester $(\mathrm{C}-\mathrm{O})$ stretching; and d) aliphatic $\left(\mathrm{C}-\mathrm{H}_{3}\right.$ and $\left.\mathrm{C}-\mathrm{H}_{2}\right)$ bending vibrations; e) hydroxyl stretching $(\mathrm{O}-\mathrm{H}) ;$ f) carbonyl $(\mathrm{C}=\mathrm{O})$ and ester $(\mathrm{C}-\mathrm{O})$ stretching; and g) aliphatic $\left(\mathrm{C}-\mathrm{H}_{2}\right.$ and $\left.\mathrm{C}-\mathrm{H}_{2}\right)$ bending vibrations. All spectra normalized by peak area to $\delta_{\mathrm{s}}$ at $1377 \mathrm{~cm}^{-1}$. 


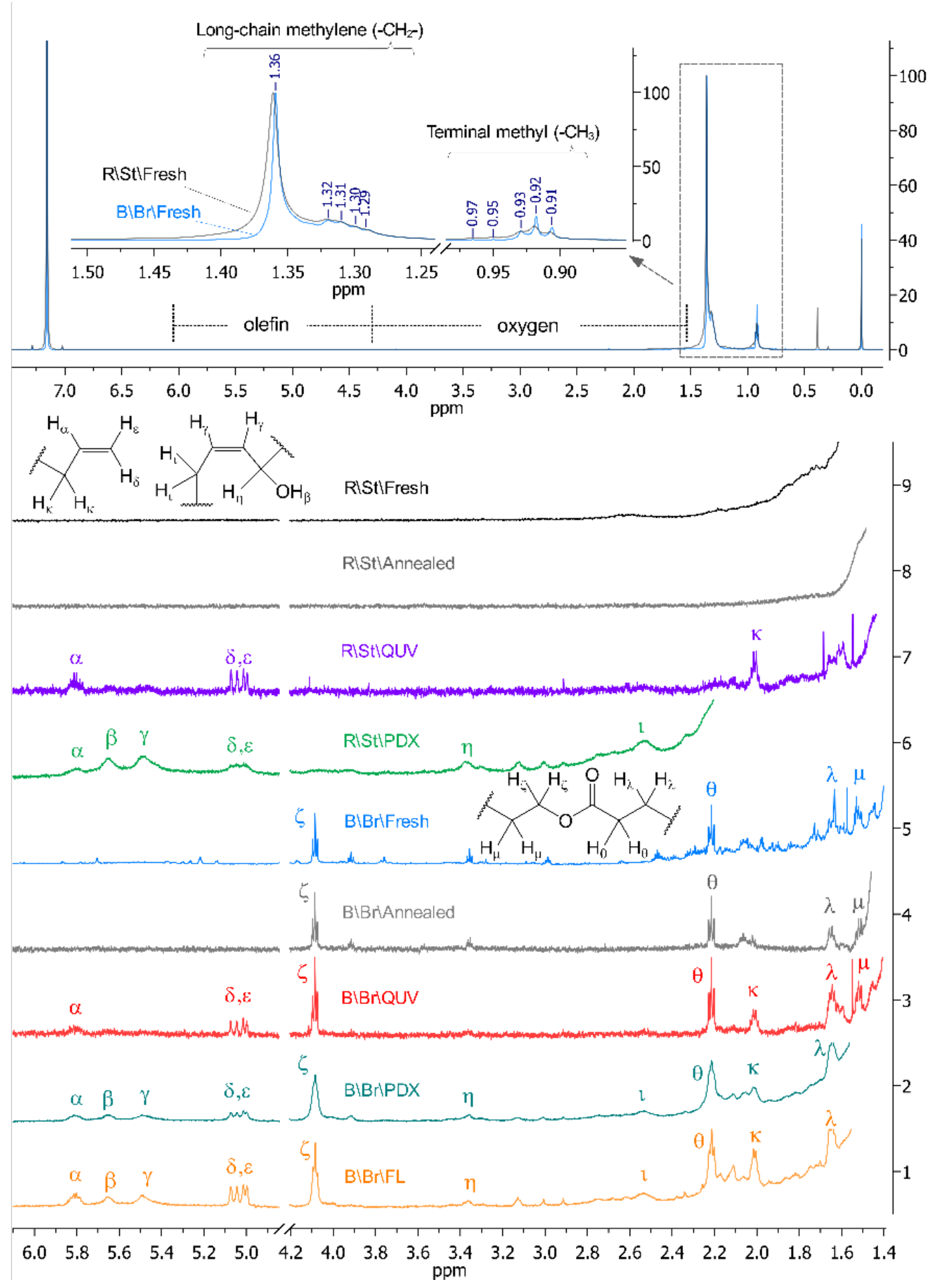

Figure 2.5 ${ }^{1} \mathrm{H}$ NMR spectra from Renaissance and Butcher's waxed panels, a) full ${ }^{1} \mathrm{H}$ NMR spectrum of the fresh waxes before weathering and $b$ ) both waxes after annealing or weathering. Peaks assigned in Table 2.3 are represented $\alpha-\mu$ and the corresponding protons notated on the chemical structures of a vinyl, unsaturated alcohol and fatty acid alkyl ester. 
Table 2.2. Characterization of significant IR absorption peaks in waxes

\begin{tabular}{|c|c|c|c|}
\hline \multirow{2}{*}{$\begin{array}{l}\text { Peak } \\
\left(\mathrm{cm}^{-1}\right)\end{array}$} & \multicolumn{3}{|c|}{ Functional group } \\
\hline & Vibration & assignment & Intensity \\
\hline (3400) & $(\mathrm{O}-\mathrm{H} s t)$ & (hydroxyl) & $(0.008-0.313)$ \\
\hline$(3076)$ & $(H-C=C s t)$ & (C-H vinyl) & $(0.002-0.008)$ \\
\hline 2955 & $\mathrm{C}-\mathrm{H}$ st & $\mathrm{C}-\mathrm{H}_{3}$ vas & 0.1 \\
\hline 2918 & $\mathrm{C}-\mathrm{H}$ st & $\mathrm{C}-\mathrm{H}_{2}$ vas & 1 \\
\hline 2872 & $\mathrm{C}-\mathrm{H}$ st & $\mathrm{C}-\mathrm{H}_{3}$ vs & 0.09 \\
\hline 2849 & $\mathrm{C}-\mathrm{H}$ st & $\mathrm{C}-\mathrm{H}_{2}$ vs & 0.68 \\
\hline (1784) & $(C=O s t)$ & (peracid) & $(0.004-0.022)$ \\
\hline 1737 & $\mathrm{C}=\mathrm{O}$ st & ester or aldehyde & 0.041 \\
\hline 1721 & $C=O$ st & ketone & 0.027 \\
\hline 1713 & $\mathrm{C}=\mathrm{O}$ st & carboxylic acid & 0.26 \\
\hline (1699) & $(C=O s t)$ & (free fatty acid) & $(0.013-0.031)$ \\
\hline$(1640)$ & $(C=C s t)$ & $(C=C$ vs $)$ & $(0.006-0.013)$ \\
\hline 1473 & $\mathrm{C}-\mathrm{H}$ bend & $\mathrm{C}-\mathrm{H}_{3} \delta \mathrm{as}$ & 0.094 \\
\hline 1463 & $\mathrm{C}-\mathrm{H}$ bend & $\mathrm{C}-\mathrm{H}_{2}$ Sas scissor & 0.109 \\
\hline 1413 & $\mathrm{C}-\mathrm{H}$ bend & $\mathrm{CH}_{2} \mathrm{C}(=\mathrm{O}) \mathrm{CH}_{2}$ & 0.008 \\
\hline 1377 & $\mathrm{C}-\mathrm{H}$ bend & $\mathrm{C}-\mathrm{H}_{3} \delta \mathrm{s}$ & 0.013 \\
\hline$(1170)$ & (C-O-C st) & (ester) & $(0.016-0.021)$ \\
\hline 1168 & C-O st & $\mathrm{C}-\mathrm{OH}$ or $\mathrm{C}-\mathrm{O}$ & 0.012 \\
\hline$(1163)$ & $(O-C=O s t)$ & (ester and acid) & $(0.004-0.067)$ \\
\hline$(908)$ & $(H-C=C$ bend $)$ & (terminal vinyl oop) & $(0.005-0.024)$ \\
\hline 730 & $\mathrm{C}-\mathrm{H}_{2}$ bend & long chain methylene & 0.052 \\
\hline 720 & $\mathrm{C}-\mathrm{H}_{2}$ bend & long chain methylene & 0.056 \\
\hline
\end{tabular}

weathering. Key spectral characteristics of the waxes before weathering are listed in Tables 2.2-2.3 and included mostly the aliphatic portions, from methyl $\left(-\mathrm{CH}_{3}\right)$ and methylene $\left(-\mathrm{CH}_{2}-\right)$ functional groups of both waxes, and some oxidized species in the case of Butchers wax.

\subsubsection{Aliphatic changes through chain-scission and cross-linking}

Using Peak Areas (PA), the relative amounts of: (1) methyl, from PA $1377 \mathrm{~cm}^{-1}$, (2) terminal vinyl, from PA $908 \mathrm{~cm}^{-1}$ and (3) methylene doublet bands, from the sum of PA $730 \mathrm{~cm}^{-1}+$ PA $719 \mathrm{~cm}^{-1}$ were compared before and after weathering. Before weathering the $\mathrm{CH}_{2}: \mathrm{CH}_{3}$ ratio calculated for Renaissance wax (fresh=6.2, annealed $=6.5$ ) was similar to Butcher's wax (fresh=5.9, annealed=5.7), and neither appreciably changed upon 
annealing. All samples showed increases in the $\mathrm{CH}_{2}: \mathrm{CH}_{3}$ ratio with weathering (though the change was not statistically significant for either wax weathered outdoors). Such comparisons of methylene to methyl ratios can also be obtained from NMR spectra where the integrated peak ratios $\int \mathrm{H}_{\mathrm{CH}_{2}}: \int \mathrm{H}_{\mathrm{CH}_{3}}$ showed larger values upon annealing: Renaissance $($ fresh $=6.99$, annealed $=7.57$ ) and for Butcher's $($ fresh $=5.95$, annealed $=$ 8.53). After weathering, relative changes from the annealed PA ratios were used to monitor chain scission (a decrease) or cross-linking (an increase) reactions. For Renaissance wax, the largest relative increase in aliphatic ratio by either method was observed on the OUV weathered steel panel, which was higher than the same type of wax and weathering on bronze. For Butcher's wax, the bronze samples weathered outdoors in FL showed the largest increases in the aliphatic ratio by $\mu$ FTIR and by NMR,

Table 2.3. ${ }^{1} \mathrm{H}$ NMR character table of S. Florida-weathered Butcher's wax on bronze

\begin{tabular}{|c|c|c|c|c|c|}
\hline $\begin{array}{l}\text { Chemical } \\
\text { shift (ppm) }\end{array}$ & $\begin{array}{l}\text { Splitting } \\
\text { pattern }\end{array}$ & $\begin{array}{l}\text { Coupling } \\
\text { Constant }(\mathrm{Hz})\end{array}$ & Proton assignment & $\begin{array}{l}\text { Chemical species } \\
\text { assignment }\end{array}$ & $\begin{array}{l}\text { Spectral } \\
\text { label }\end{array}$ \\
\hline 0.00 & singlet & & $-\mathrm{CH}_{3}$ & TMS, shift reference & \\
\hline 0.40 & singlet & & H-DO & HDO (deuterated water) & \\
\hline 0.92 & triplet & $6.97,6.97$ & $-\mathrm{CH}_{3}$ & Methyl (terminal) & \\
\hline 1.34 & multiplet & & $-\mathrm{CH}_{2}^{-}$ & Long-chain methylene & \\
\hline 1.52 & triplet & $6.73,6.82$ & $-\mathrm{CH}_{2}-$ & Fatty-acid alkyl ester & $\mathrm{m}$ \\
\hline 1.65 & triplet & $7 \cdot 5,7 \cdot 4$ & $-\mathrm{CH}_{2}-$ & Fatty-acid alkyl ester & I \\
\hline 2.01 & multiplet & & $-\mathrm{CH}_{2}-\mathrm{CH}=\mathrm{CH}_{2}$ & Vinyl (terminal) & k \\
\hline 2.21 & triplet & $7 \cdot 46,7.46$ & $-\mathrm{CH}_{2}-\mathrm{O}-\mathrm{C}(=\mathrm{O})-\mathrm{CH}_{2}-$ & Fatty-acid alkyl ester & $q$ \\
\hline 2.54 & multiplet & & $-\mathrm{CH}_{2}-\mathrm{CH}=\mathrm{CH}-\mathrm{CH}(\mathrm{OH})-\mathrm{CH}_{2}-$ & $\alpha, \beta$-unsaturated $2^{\circ}$ alcohol & $\mathrm{i}$ \\
\hline $3 \cdot 36$ & multiplet & & $-\mathrm{CH}_{2}-\mathrm{CH}=\mathrm{CH}-\mathrm{CH}(\mathrm{OH})-\mathrm{CH}_{2}-$ & $\alpha, \beta$-unsaturated $2^{\circ}$ alcohol & $\mathrm{h}$ \\
\hline 4.09 & triplet & $6.76,6.76$ & $-\mathrm{CH}_{2}-\mathrm{O}-\mathrm{C}(=\mathrm{O})-\mathrm{CH}_{2}-$ & Fatty-acid alkyl ester & z \\
\hline 5.01 & doublet & 9.83 & $-\mathrm{CH}=\mathrm{C}(\mathrm{H})-\mathrm{H}_{\mathrm{cis}}$ & Vinyl (terminal) & e \\
\hline 5.06 & doublet & $15 \cdot 7$ & $-\mathrm{CH}=\mathrm{C}(\mathrm{H})-\mathrm{H}_{\text {trans }}$ & Vinyl (terminal) & $d$ \\
\hline 5.49 & multiplet & & $-\mathrm{CH}_{2}-\mathrm{CH}=\mathrm{CH}-\mathrm{CH}(\mathrm{OH})-\mathrm{CH}_{2}-$ & $\alpha, \beta$-unsaturated $2^{\circ}$ alcohol & $\mathrm{g}$ \\
\hline 5.65 & multiplet & & $-\mathrm{CH}_{2}-\mathrm{CH}=\mathrm{CH}-\mathrm{CH}(\mathrm{OH})-\mathrm{CH}_{2}-$ & $\alpha, \beta$-unsaturated $2^{\circ}$ alcohol & $\mathrm{b}$ \\
\hline 5.82 & multiplet & & $-\mathrm{CH}=\mathrm{CH}_{2}$ & Vinyl (terminal) & $\mathrm{a}$ \\
\hline 7.16 & & & Aromatic & Benzene (solvent) & \\
\hline
\end{tabular}


although the sample weathered by QUV also showed a large increase. The general trend of increasing methylene to methyl ratios suggested that cross-linking is a common type of reaction that occurred with weathering of both waxes.

Offering further insight into the weathering induced changes is the PA ratio of the (terminal) vinyl to methyl groups shown in Figures $2.4 \mathrm{~d}, 2.4 \mathrm{~g}$ and listed in Table 2.4, where QUV weathered panels showed larger increases in vinyl character than outdoor weathered panels. An increase in the vinyl to methyl peak area ratios could be an indicator of chain scission. Using mFTIR, vinyl bands were only observed in the OUV weathered Butcher's wax after weathering (as can be seen in Fig. 2.4f), but were detected in both waxes by NMR after every weathering method by a peak corresponding to the vinylidene proton $\left(-\mathrm{CH}=\mathrm{CH}_{2}\right)$ at $5.82 \mathrm{ppm}$ and two doublets representing the vinyl protons $\left(-\mathrm{CH}=\mathrm{CH}_{2}\right)$ at 5.06 and $5.01 \mathrm{ppm}$ (as seen in Figure 2.5 and Table 2.3).

Table 2.4. Peak area ratio from $\mu$-FTIR and ${ }^{1} \mathrm{H}$ NMR data of waxes

\begin{tabular}{|c|c|c|c|c|c|}
\hline & $\begin{array}{l}\mathrm{CH}_{2}: \mathrm{CH}_{3} \mathrm{PA} \\
\frac{\left(730+719 \mathrm{~cm}^{-1}\right)}{\left(1377 \mathrm{~cm}^{-1}\right)}\end{array}$ & $\begin{array}{l}\int \mathbf{H}_{\mathbf{C H}_{\mathbf{2}}}: \int \mathbf{H}_{\mathbf{C H}_{\mathbf{3}}} \\
\underline{\text { (1.34 ppm) }} \\
\text { (0.92 ppm) }\end{array}$ & $\begin{array}{l}\mathrm{H}-\mathrm{C}=\mathrm{CH}_{2}: \mathrm{CH}_{3} \\
\text { PA } \\
\frac{\left(908 \mathrm{~cm}^{-1}\right)}{\left(1377 \mathrm{~cm}^{-1}\right)}\end{array}$ & $\begin{array}{l}\text { Unsat'd \% } \\
\frac{\int \mathrm{H}_{\mathrm{C}=\mathrm{C}} \times 100 \%}{\int \mathrm{H}_{\text {total }}}\end{array}$ & $\begin{array}{l}\text { Oxidized \% } \\
\frac{\int \mathrm{H}_{\mathrm{Ox}} \times 100 \%}{\int \mathrm{H}_{\text {total }}}\end{array}$ \\
\hline $\mathrm{R} / \mathrm{Br} / \mathrm{fresh}$ & $6.24 \pm 0.83$ & $6.99 \pm 0.49$ & $\mathrm{n} / \mathrm{d}$ & $0.2 \pm 0.03$ & $1.84 \pm 0.24$ \\
\hline R/Br/annealed & $6.54 \pm 0.42$ & $7.57 \pm 0.54$ & $\mathrm{n} / \mathrm{d}$ & $0.04 \pm 0.01$ & $0.77 \pm 0.10$ \\
\hline R/St/QUV $1500 \mathrm{~h}$ & $8.38 \pm 0.76$ & $8.52 \pm 0.60$ & $0.759 \pm 0.24$ & $0.67 \pm 0.09$ & $3.00 \pm 0.40$ \\
\hline $\mathrm{R} / \mathrm{Br} / \mathrm{QUV} 1250 \mathrm{~h}$ & $7.13 \pm 0.49$ & $8.24 \pm 0.58$ & $0.856 \pm 0.15$ & $1.15 \pm 0.15$ & $2.43 \pm 0.32$ \\
\hline $\mathrm{R} / \mathrm{St} / \mathrm{PDX} 36 \mathrm{mo}$ & $6.95 \pm 0.61$ & $7.88 \pm 0.56$ & $0.586 \pm 0.19$ & $1.30 \pm 0.17$ & $2.17 \pm 0.29$ \\
\hline $\mathrm{B} / \mathrm{Br} /$ fresh & $5.9 \pm 0.35$ & $5.95 \pm 0.42$ & $n / d$ & $0.46 \pm 0.06$ & $2.74 \pm 0.36$ \\
\hline B/Br/annealed & $5.74 \pm 0.37$ & $8.53 \pm 0.60$ & $\mathrm{n} / \mathrm{d}$ & $0.10 \pm 0.01$ & $1.45 \pm 0.19$ \\
\hline $\mathrm{B} / \mathrm{Br} / \mathrm{QUV} 500 \mathrm{~h}$ & $6.75 \pm 0.52$ & $9.08 \pm 0.64$ & $0.24 \pm 0.08$ & $0.52 \pm 0.07$ & $1.78 \pm 0.24$ \\
\hline $\mathrm{B} / \mathrm{Br} / \mathrm{PDX} 36 \mathrm{mo}$ & $6.81 \pm 0.66$ & $8.72 \pm 0.61$ & $\mathrm{n} / \mathrm{d}$ & $0.33 \pm 0.04$ & $2.34 \pm 0.31$ \\
\hline $\mathrm{B} / \mathrm{Br} / \mathrm{FL} 18 \mathrm{mo}$ & $7.28 \pm 0.62$ & $8.91 \pm 0.62$ & $\mathrm{n} / \mathrm{d}$ & $0.43 \pm 0.06$ & $1.71 \pm 0.23$ \\
\hline
\end{tabular}

Samples in the left column are abbreviated as follows: $\mathrm{B}=$ Butcher's wax, $\mathrm{R}=$ Renaissance wax, $\mathrm{Br}=$ bronze, St $=$ steel, QUV = accelerated UV-B, PDX = Portland, Oregon, and FL = South Florida. PA = peak area, $\mathrm{H}_{\mathrm{Ox}}=$ protons from 4.1-1.5 $\mathrm{ppm}, \mathrm{H}_{\mathrm{C}=\mathrm{C}}=$ protons from 6.0-4.3 $\mathrm{ppm}, \mathrm{H}_{\text {total }}=$ all wax protons, $\mathrm{n} / \mathrm{d}$ = not detected 
While the same vinyl group NMR pattern was observed to increase in intensity with weathering in both types of waxes and under all weathering conditions, additional olefin characteristics were seen in the panels weathered outdoors. The outdoorweathered Renaissance and Butcher's waxed panels contained the highest percentage of mid-chain $\alpha$,b-unsaturated alcohols as exhibited by two strong peaks at 5.65 and 5.49 ppm in the NMR spectra. The percent unsaturation was calculated by integrating the ${ }^{1} \mathrm{H}$ NMR peaks in the olefin region (6.0-4.3 ppm) relative to the sum of all waxrelated peaks in each spectrum in Figure 2.5 , with the percentages listed in Table 2.4 . While pure paraffinic waxes do not directly absorb in the UV, oxidation of a few sites in the alkane chains may occur during chemical processing of the wax, making those sites susceptible to photochemical cleavage reactions. ${ }^{99}$ The products of such cleavages may produce terminal vinyl moieties (and methylene radicals), which can participate in further reactions, including cross-linking, termination, or further oxidation.

\subsubsection{Oxidation and fatty acid alkyl esters}

Oxygen-containing functional groups were initially observed only in the fresh Butcher's wax by the peaks in the mFTIR spectra in Figure $2.4 \mathrm{a}: \mathrm{C}=\mathrm{O}$ from $1740-1710 \mathrm{~cm}^{-1}, \mathrm{C}-\mathrm{O}$ at $1168 \mathrm{~cm}^{-1}$, and $-\mathrm{CH}_{2}-\mathrm{C}(=\mathrm{O})-\mathrm{CH}_{2}-$ at $1413 \mathrm{~cm}^{-1}$ and by NMR in Figure 2.5a: triplets at 4.09, 2.21, 1.65, and $1.52 \mathrm{ppm}$ were assigned to fatty acid alkyl esters. The percent oxidation by NMR was calculated by integrating the oxygen-containing range (4.1-1.5 ppm) over the sum of all wax peaks in each spectrum in Figure 2.5. The results in Table 2.4 showed that fresh Renaissance wax contained fewer oxidized hydrocarbons (1.8\%) 
than Butcher's wax (2.7\%) and that percentage was reduced after annealing to $0.8 \%$ and $1.5 \%$, respectively, suggesting that some of the oxidized hydrocarbon species were volatile.

The Table 2.4 results also indicate that every weathered waxed panel contained a higher percentage of oxidized species than the respective annealed panel, suggesting that oxidation of these waxes is a common feature of weathering. For Renaissance waxed substrates, the mFTIR spectral signature of those products included the appearance of carbonyl bands at $1784 \mathrm{~cm}^{-1}, 1736 \mathrm{~cm}^{-1}, 1726 \mathrm{~cm}^{-1}$, and $1699 \mathrm{~cm}^{-1}$ where none existed prior to weathering as can be seen by comparing the spectra in Figure 2.4c. And from the NMR spectra in Figure 2.5, fresh and annealed Renaissance wax showed the presence of mostly alkanes (with only traces of oxygen-containing functional groups), then upon weathering more intense peaks appeared in the oxidized region including peaks corresponding to $\alpha, b$-unsaturated alcohols. Comparing the spectral differences between outdoor and accelerated weathering showed that panels weathered in Portland, Oregon preferentially oxidized to ketones, acids and alcohols, while the weathered QUV panels showed larger amounts of more highly oxidized hydrocarbons in the form of ester and peracid bands.

For Butcher's wax, specific chemical changes after weathering included the presence of new hydroxyl peaks, conversion of the broad ether band to a sharp ester peak at $1170 \mathrm{~cm}^{-1}$ coupled with an increase in the carbonyl peak at $1737 \mathrm{~cm}^{-1}$ and an increase in the relative intensity of methyl groups (Figs. 2.4e-g). Together, those changes indicate the hydrolysis of ester linkages in long-chains to produce fatty acid 
alkyl esters. The existence of fatty acids was verified by NMR, with four triplet peaks identified in Table 2.3 and Figure 2.5 $\mathrm{b}$ at $4.09 \mathrm{ppm}\left(\mathrm{H}_{\mathrm{D}-\mathrm{G}}, \mathrm{J}_{\mathrm{D}}=6.8 \mathrm{~Hz}\right), 2.21 \mathrm{ppm}\left(\mathrm{H}_{\mathrm{E}-\mathrm{F}_{1}} \mathrm{~J}_{\mathrm{E}}=7.5\right.$ $\mathrm{Hz})$, $1.65 \mathrm{ppm}\left(\mathrm{H}_{\mathrm{F}-\mathrm{E},} \mathrm{J}_{\mathrm{F}}=7.4 \mathrm{~Hz}\right)$, and $1.52 \mathrm{ppm}\left(\mathrm{H}_{\mathrm{G}}, \mathrm{J}_{\mathrm{G}-\mathrm{D}}=6.7 \mathrm{~Hz}\right)$. Subsequently, the intensity of the lower wavenumber ketone/acid bands $\left(1720-1700 \mathrm{~cm}^{-1}\right)$ decreased during weathering and it seems likely that those groups were: (1) converted into esterified product during weathering, (2) were lost as a result of backbone cleavage into volatile small molecules or (3) underwent keto-enol tautomerization leading to mid-chain unsaturation. Because increases in methylene to methyl peak areas were observed with weathering (shown in Table 2.4), taken together with these data, a picture emerges that suggests a competing reaction with cross-linking is fatty acid alkyl ester production. It has been suggested that the formation of cross-linked polymers and fatty acid alkyl esters are responsible for the difficulty in removing aged waxes from metal substrates as both products are less soluble in many common solvents. ${ }^{81}$ Additionally, the breakdown of oxidized groups such as those in fatty acid alkyl esters can promote the onset of metal corrosion through the formation of metal soaps. ${ }^{100}$

Photographic documentation of the panels showed that weathering rapidly altered the visual appearance of the films, where thinning, flaking and cracking was readily observed (before and after micrographs of the panels are shown in Appendix B1). In addition to the unique chemical species detected in the films after weathering, it is likely that significant quantities of volatile low molecular weight species evaporated during weathering due to chain-scission and were not detected. While determining breakdown mechanisms of these wax films is outside the scope of this work, others 
have suggested that terminal reactive species form during cleavage of the polyethylene backbone. ${ }^{85-86,101}$ This pathway can lead to products observed in our data: (1) crosslinking, suggested by increase in aliphatic PA (observed in both waxes), (2) chain scission from appearance of terminal vinyl groups, suggested by new $\mathrm{H}-\mathrm{C}=\mathrm{C}$ peaks (observed in both waxes) and (3) production of fatty acid alkyl esters, proposed by tandem increases in $\mathrm{C}=\mathrm{O}$ and $\mathrm{C}-\mathrm{O}$ peaks (observed only for Butcher's wax).

\subsection{Loss of film thickness and corrosion}

The ratio of peak intensities of wax by TF-XRD was used to monitor the relative loss of the wax (such as by ablation, flaking, etc.) versus loss of the metal (such as by corrosion) due to weathering. PA ratio data is shown in Table 2.5 with film thickness gauge measurements provided for comparison. In general, the most wax was lost and the greatest amount of corrosion occurred during outdoor weathering, as those panels gave the smallest wax-to-metal ratios. It is likely that waxed panels weathered by OUV performed the best by this metric (showed the smallest change in ratio) due to the lack of ablative particles and absence of corrosive salts that outdoor panels experienced. In the case of steel substrates weathered in Florida, the wax film and much of the metal panel was lost entirely to corrosion by six months of weathering. Measurements of film thickness using a thickness gauge were likely inaccurate because of corrosion between the wax and metal substrate that changed over time, but are included in Table 2.5 since those measurements are commonly reported. 
Corrosion products were identified in the TF-XRD spectra of weathered bronze and steel substrates, and are discussed in more detail in Appendix C. Sulfate and ammonium derived corrosion products were only present on substrates having an

Table 2.5. TF-XRD characterization and film thickness gauge measurements of wax remaining after weathering

\begin{tabular}{lccc}
\hline & $\mathrm{PA}_{\text {wax }}: \mathrm{PA}_{\text {metal }}$ & $\% \Delta \mathrm{PA}$ & $\% \Delta$ Film thickness $(\mu \mathrm{m})$ \\
\hline B/Br/before & $0.215 \pm 0.005$ & & \\
B/Br/QUV 500 h & $0.150 \pm 0.0037$ & -30.2 & -37.5 \\
B/Br/PDX 36 mo & $0.126 \pm 0.0033$ & -41.4 & +1.24 \\
B/Br/FL 18 mo & $0.102 \pm 0.0035$ & -52.6 & -33.4 \\
R/Br/before & $0.517 \pm 0.022$ & & \\
R/Br/QUV 1250 h & $0.394 \pm 0.0082$ & -23.8 & -21.8 \\
R/St/before & $0.520 \pm 0.020$ & & -56.1 \\
R/St/QUV 1250 h & $0.130 \pm 0.009$ & -75.0 & -27.8 \\
R/St/PDX 36 mo & $0.087 \pm 0.007$ & -83.3 & \\
R/St/FL 6 mo & $\mathrm{n} / \mathrm{d}$ & & \\
\hline
\end{tabular}

$\mathrm{PA}_{\text {wax }}$ : $\mathrm{PA}_{\text {metal }}$ calculations used the peak area of wax at $21.52 \theta$ to the metal at 42.9 $2 \theta$ for bronze or $64.92 \theta$ for steel (from the spectra in Appendix C). Samples in the left column are abbreviated as follows: $\mathrm{R}=$ Renaissance wax, $\mathrm{B}=$ Butcher's wax, $\mathrm{Br}=$ bronze, St = steel, QUV =UV-B with condensation, $\mathrm{PDX}=$ Portland, Oregon, and FL = South Florida. PA = peak area. $\% \Delta$ = percent change (in measured parameter) after weathering

applied patina but were detected on both bronze and steel panels, likely because the patination solution contained ammonium molybdate, cupric sulfate and zinc sulfate. For steel the dominant peaks were attributed to goethite $[\alpha-\mathrm{FeOOH}]$, akaganeite $[\beta-$ $\mathrm{FeOOH}]$, lepidocrocite $[\gamma-\mathrm{FeOOH}]$, and ammonium iron sulfate $\left[\left(\mathrm{NH}_{4}\right)_{3} \mathrm{Fe}\left(\mathrm{SO}_{3} \mathrm{O}\right)_{3}\right]$. While the corrosion peaks were small, spectral matching suggested that the identities of the copper-containing corrosion products detected on bronze substrates were: cuprite $\left[\mathrm{CU}_{2} \mathrm{O}\right]$, atacamite $\left[\mathrm{CU}_{2}(\mathrm{OH})_{3} \mathrm{Cl}\right]$ and a basic copper sulfate hydroxide $\left[\mathrm{CU}_{2.5} \mathrm{~S}_{4}(\mathrm{OH})_{3} \cdot 2 \mathrm{H}_{2} \mathrm{O}\right]$. Not surprisingly, the chloride corrosion products were produced most abundantly in the S. Florida exposures because the panels were sprayed with salt water periodically (to provide a more aggressive weathering environment). The beta 
structure of akaganeite is typical of aggressive atmospheres since it requires the aid of an ion (like chloride) to form and was found only in samples that had been weathered outdoors. Lepidocrocite is formed in weakly acidic environments and was present in the largest quantity for the Florida weathered steel. While fatty acid alkyl esters were detected by IR and NMR on multiple substrates, their metal salts were not observed by X-ray diffraction, likely because they were poorly crystalline.

\subsection{EIS Evaluation of Weathered Waxes}

EIS data were acquired periodically during weathering to observe changes in the barrier properties of the films. The spectra at the start of the experiments were fit using the circuit model labeled 'Before' in Figure 2.1. A representative initial EIS spectrum of each wax is shown in Figure $2.3 \mathrm{~b}$ and is typical of a mostly intact coating that has small pores. The capacitance of the coating $\left(\mathrm{CPE}_{\text {coat }}\right)$ dominates at high frequencies (from 10 $\mathrm{kHz}$ to $1 \mathrm{MHz}$ ) and its presence is an indication of a good protective coating, while observation of another capacitor, the double layer capacitance $\left(\mathrm{CPE}_{\mathrm{dl}}\right)$ at lower frequencies (from $1 \mathrm{~Hz}$ to $10 \mathrm{kHz}$ ), is an indication that the coating has pores. A third capacitor can be assigned to an oxide layer $\left(\mathrm{CPE}_{\mathrm{ox}}\right)$, from patina or corrosion, and appears in mid-range frequencies $(1 \mathrm{~Hz}$ to $10 \mathrm{kHz})$ in the spectrum if present at all.

Based on spectral similarities observed during the course of weathering, the data suggest that all waxed panels aged in a few discrete steps, which are labeled as stages in Figure 1 and Figure 2.6. The EIS stages in Figure 2.6 were determined by: (1) determining an acceptable range of time constants $\left(T_{R C}\right)$, calculated from the product 

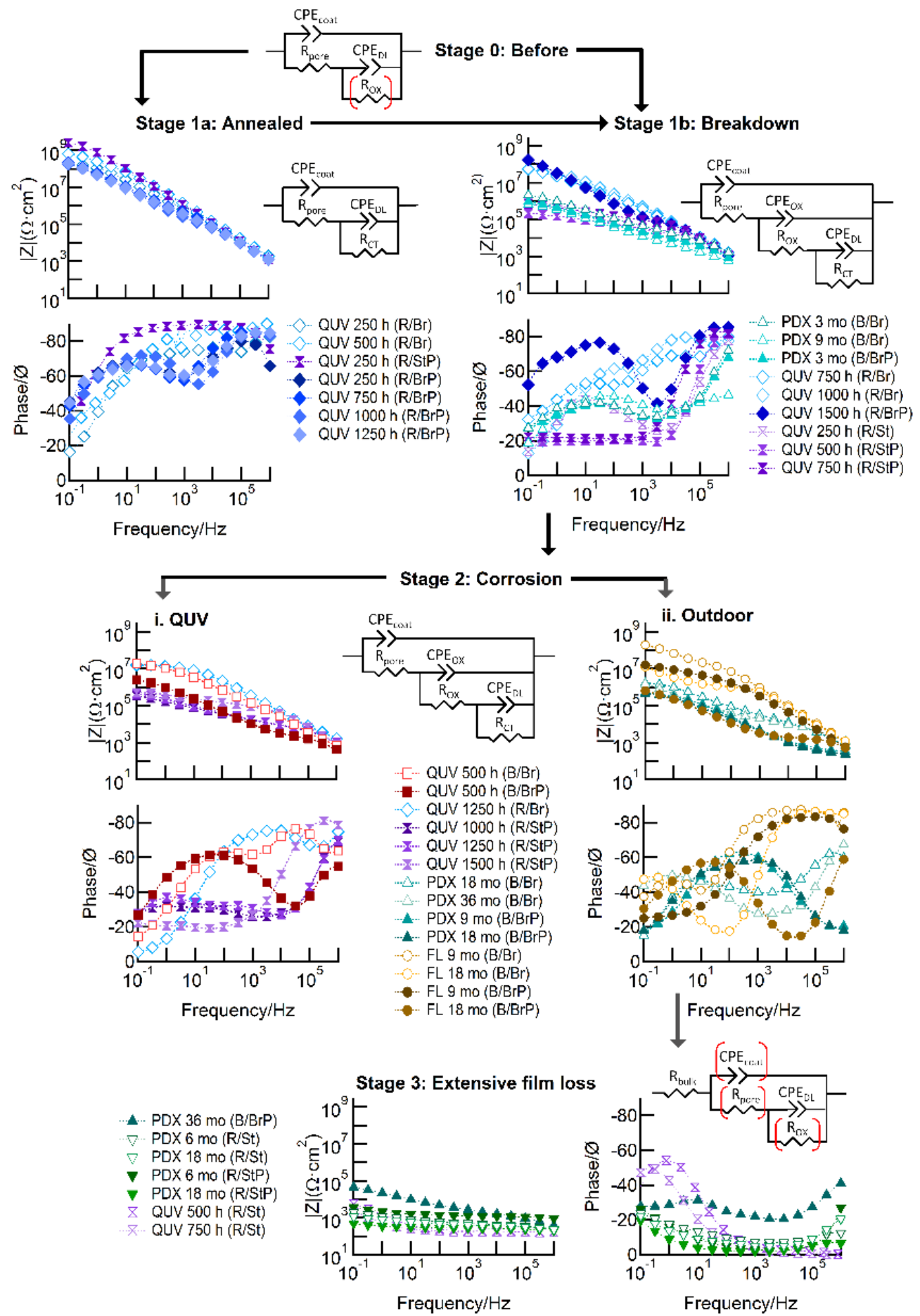

Figure 2.6 EIS Bode plots from all waxed samples after weathering. Equivalent circuit models and fit values were used to match sample time points to various key stages in the coating lifetime illustrated in Figure 1. Circuit elements in parenthesis were either not observed or did not dominate the spectrum at frequencies measured. A detailed explanation of this Figure is given in Appendix D: Detailed analysis of impedance spectra of wax coated substrates. 
of pore resistance $\left(R_{\text {pore }}\right)$ and coating capacitance $\left(C_{\text {coat }}\right)$, (2) observing the magnitude of the relative changes in total impedance, (3) observing the presence of and a threshold magnitude of oxide layer resistance $\left(R_{\mathrm{ox}}\right)$. We reserved the detailed analysis of the coatings' progress through each stage for Appendix D. In some cases, the coated panels rapidly moved through the stages without observation of any intermediate stages, so that the first data point taken after the start of weathering was equal to the final stage (coating failure); this was the case for all waxed steel panels that were weathered outdoors.

The change in the values of some of the EIS spectral features plotted over time are shown in Figure 2.7. Specifically, relative to their starting value, the value of coating capacitance, pore resistance and RC time constant of the coating were individually extracted from the EEC models in Figure 2.6 and the total impedance of the spectra is reported at $0.1 \mathrm{~Hz}$ at different points in weathering time. This analysis showed that independent of weathering method, alloy type, or presence/absence of patina, $C_{\text {coat }}$ increased for all waxed panels with exposure time. Typically, after 9 to 18 months of outdoor exposure, the coating capacitance had increased by nearly one order of magnitude. Even the panel that showed the least impedance change during weathering, which was the Butcher's waxed plain bronze panel (B/BR PDX), showed an increase in $\mathrm{C}_{\text {coat }}$ by two orders of magnitude after 36 months; however extensive corrosion was observed under the wax coating by that time. Overall, observed capacitance changes were smaller for the panels having undergone accelerated weathering compared to outdoor weathering, which may be due to increased cross- 
linking reactions during QUV weathering (that were discussed in section 2.6.1). Most commonly $R_{\text {pore }}$ decreased over time, but on occasion was seen to increase, likely due

a) Coating capacitance
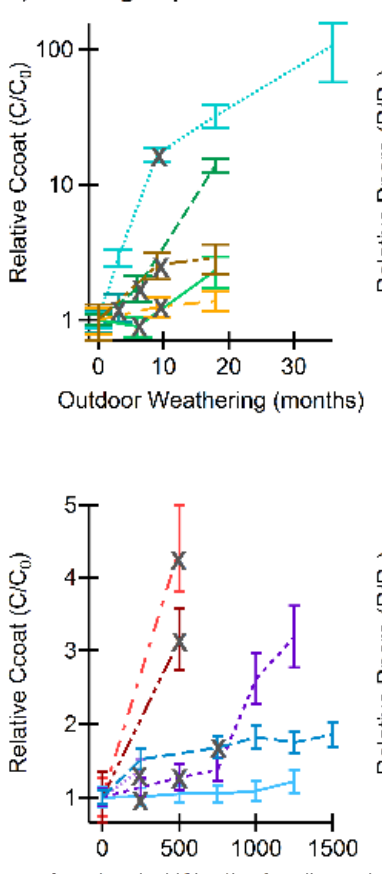

Accelerated Weathering (hours) b) Pore resistance

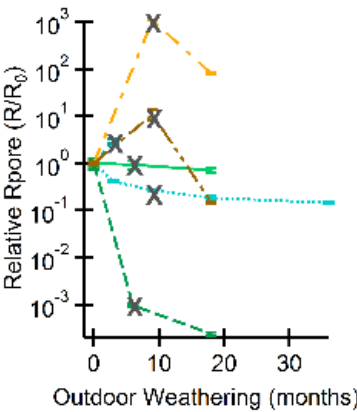

- R/St PDX

--- R/StP PDX

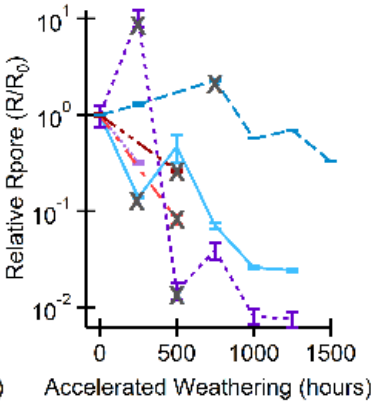

Accelerated Weathering (hours)

-. - B/Br QUV

-... B/BrP QUV c) Total impedance

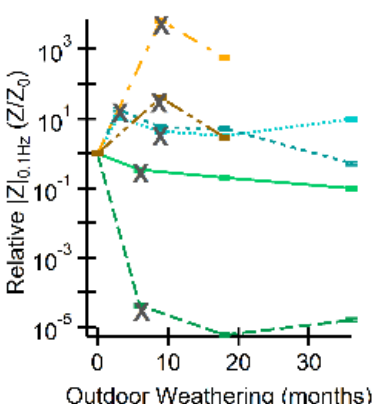

Outdoor Weathering (months)

$$
\begin{array}{ll}
\mathrm{B} / \mathrm{Br} \mathrm{PDX} & -\cdots \mathrm{B} / \mathrm{BrFL} \\
\mathrm{B} / \mathrm{BrPPDX} & -\cdots \mathrm{B} / \mathrm{BrPFL}
\end{array}
$$
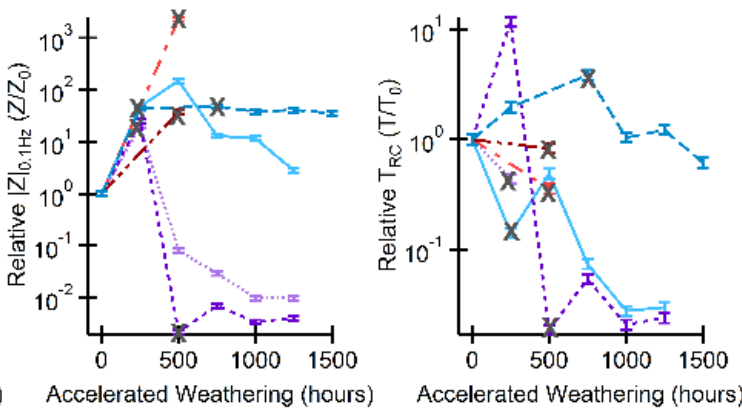

- R/Br QUV

-- R/BrP QUV d) RC time constant

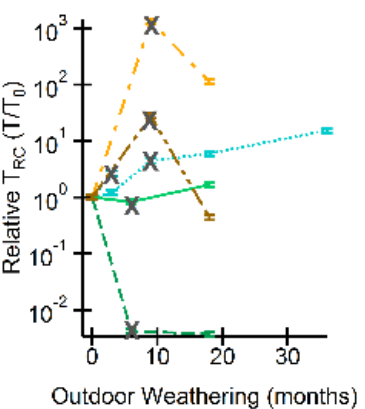

Figure 2.7 Extracted circuit elements from fitting EEC models to EIS spectra (Bode/Nyquist plots) plotted over time relative to initial values: a) coating capacitance, b) pore resistance, c) total impedance at low frequencies, and d) $\mathrm{RC}_{\text {coat }}$ time constant of protective coating; outdoor weathering data shown in the top row and QUV-B data shown in the bottom row. Photographs of the panels at time points marked $(X)$ are included in Appendix B: Micrographs and photographs of weathered wax samples. The time sequences shown ended when the respective element was removed from the EEC.

to the formation of an oxide layer at the bottom of the pore (shortening the path length through the pore). This was observed on waxed plain and patinated bronze panels after 9 months of weathering in Florida and patinated bronze and steel after weathering in the QUV chamber for 750 and $250 \mathrm{~h}$, respectively. The pore resistance of those four panels decreased after the stated time points. For all other types of weathering, $R_{\text {pore }}$ decreased from its initial $(t=0)$ value, most likely due to an increase in the porosity of 
the film. Under the elevated temperatures of the OUV weathering chamber, the wax film was able to soften and fill in defects, which likely explains the observed increase in $R_{\text {pore }}$ that was only seen in that method of weathering. The high impedance at $0.1 \mathrm{~Hz}$ (plotted over time in Figure 2.7c) that was observed after weathering is related to the balance of which RC pair dominates ( $T_{\text {coat }}$ is plotted over time in Figure 2.7d). Total impedance of wax coatings investigated predominately followed the same trend of pore resistance $\left(R_{\text {pore }}\right)$ with some contribution from oxide growth $\left(C_{o x} / R_{o x}\right)$. Those latter elements became dominant when the capacitance of the coating increased (which registers as a decrease in impedance, since capacitance and impedance are inversely proportional) as the coating became more defective. Photographs of each panel are shown in Appendix B2.

While the total impedance at frequencies less than $1 \mathrm{~Hz}$ has been used as a measure of the quality of a coating's protective nature, ${ }^{21,102}$ in this work we observed the opposite effect due to the increasingly dominant contributions of other elements that are markers of film degradation and substrate corrosion. Our interpretation of the spectral changes observed (independent of substrate type or patination) after weathering is: (1) electrolyte uptake into an increasingly defective coating leads to an increase in coating capacitance, (2) formation of pores in the film results in a decrease in the resistance of $R_{\text {pore, }}$ and (3) growth of corrosion on the substrate results in an increase in total impedance, $|Z|$. Given the significant spectral changes observed after such short exposure periods as 9-18 months weathering outdoors in Portland and by 9 months (the first time point after exposure) in Florida, wax films, even on bronze, failed 
to offer protection against corrosion. As previously stated, the wax' lifetime was even shorter on steel substrates. Comparing this EIS data to the chemical changes observed, it is likely that some of the changes in barrier properties of the film were due to the oxidative changes and chain-scission observed.

\subsection{Patination}

The effect of the patina on the impedance of waxed bronze and steel was investigated by comparing performance of each type during weathering. Impedance spectra of patinated substrates showed more variation after outdoor weathering than was seen for plain substrates, possibly due to formation of a secondary patina of less stable corrosion products. In contrast, we observed that patinas have a stabilizing effect during accelerated weathering as those samples showed less variation in the impedance spectra and because the point of failure was twice as long for patinated bronze (plain $=500$ hours vs. patina $=1250$ hours $)$ and for patinated steel $($ plain $=250 \mathrm{~h}$ vs. patina $=500 \mathrm{~h})$, when the lifetime was determined by the time to transition to Stage Ib (from Stage 1a). The apparent protective effect of patinas was seen as: an increase (between 250-500 h) in $R_{\text {pore }}$ coupled with an increase in charge-transfer resistance, $R_{c t}$ (higher barrier to conversion of the metal to oxide). Conversely in the case of outdoor weathering, it is likely that mineral species formed during patination participate in further corrosion reactions when exposed to salts and pollutants in an outdoor environment. Lattice mismatch between the corrosion species/patina and the underlying substrate results in the presence of voids or gaps in the corrosion layer, 
providing sites for electrolytes to induce further corrosion. Indeed, it has been noted that an artificial patina does not contribute to protection of the substrate during outdoor exposure unless the corrosion products were allowed to fully precipitate and dehydrate, but their barrier properties were still poor.$^{91}$ Conversely, while there exists evidence and data in some conservation studies that have shown careful selection of patina to weathering environment provides adequate protection of copper alloys, ${ }^{21}$ those effects were not observed here under our outdoor weathering conditions with these waxes. Because we prepared our panels in a controlled laboratory setting, we were able to take considerable care in patinating and rigorously cleaning them to remove residual patination solution. Our results suggest that these thin patinas destabilized the waxed panels weathered outdoors, which was observed by the change in magnitude in the following EIS parameters: larger relative changes in coating capacitance, decreased pore resistance, and lower total impedance compared to those same parameters from plain waxed substrates.

\subsection{Conclusions}

Our data show that waxes degrade rapidly with weathering. Based on the infrared and NMR data presented here, weathering of waxes produces cross-linked chains, oxidation products, and can result in chain-scission. These results imply that some of the reported difficulties in removing wax films may not only be due to differences in the initial molecular weight of the paraffin chains as has been proposed; ${ }^{81}$ but also to cross-linking and oxidized materials having altered solubility. Our results indicate that wax coatings 
can provide modest protection, though even the best coatings produced by the lab do not meet the minimum level of acceptable protection, which is $10 \mathrm{M} \Omega$ at $0.1 \mathrm{~Hz}{ }^{82}$ Investigations by TF-XRD indicated that annealing reduces the barrier properties of Renaissance wax because short-chains and branched alkanes migrate into amorphous regions. Due to their steric accessibility in amorphous regions, such branch points are susceptible to oxidation, chain-scission and other reactions. In contrast, annealing has a less significant effect on the structure and barrier properties of Butcher's wax as the crystalline domains are larger, making movement more constricted and chemical reactions less likely. Accelerated weathering by QUV-B, generally resulted in longer protective lifetimes compared to outdoor exposures, likely due to increased UV-induced cross-linking reactions and to the lack of ablative particles in the UV chamber. Outdoor exposure (such as under the high humidity and temperatures seen in south Florida or under the more moderate humidity and temperatures and high annual precipitation of Portland, Oregon) can lead to uneven corrosion and rapid degradation of wax films on steel substrates due to the substrate's high corrosion potential. Growth of corrosion leads to an increase in the impedance and together with photographic images, the data show that the resulting oxide layer is neither stable nor protective and that loss of substrate material is considerable. Film lifetimes, as defined by the time point before bronze panels entered stage 2, were typically between 250 and 750 hours by QUV-B, less than 9 months for panels weathered in Florida and between 9 and 18 months for panels weathered in Portland. Waxed steel panels failed before 6 months of outdoor weathering and between 250 and 750 hours of OUV-B weathering. 
Based on this study, the best practice recommendations to conservators who use waxes to protect against corrosion include: cold buffing of waxes, reapplication of waxes at an interval of less than 9 months, and ensuring that any applied patina does not lead to further corrosion (which, otherwise could further shorten the reapplication interval). Given that oxidation products were observed in aged waxes, it is likely that some of those oxidation products are capable of enhancing corrosion rates; since they are polar, more hydrophilic and particularly if they are more acidic than fresh waxes. Given those chemical changes associated with weathering, it is prudent to remove aged wax films before reapplying fresh, bearing in mind that the solubility of the aged film may be significantly altered. Certain custom blends of waxes may improve upon some of the weaknesses of these waxes if those mixtures have fewer molecules in the amorphous regions compared to their crystalline regions which would reduce their reactivity. Additionally, starting with waxes that have minimal oxidized carbon would likely minimize further oxidation reactions. These characteristics could be evaluated using similar diffraction techniques used in this study, chemical alterations studied using FTIR and NMR and their barrier properties and performance lifetimes using EIS. 


\section{Understanding the differences in film formation mechanisms of two}

comparable solvent-based and waterborne coatings on bronze substrates by electrochemical impedance spectroscopy

Adapted with permission from: Swartz, N. A.; Clare, T. L., Understanding the differences in film formation mechanisms of two comparable solvent-based and waterborne coatings on bronze substrates by electrochemical impedance spectroscopy. Electrochim. Acta 2012, 62, 199-206.

Corrosion protection of bronze, a metal alloy which is commonly used in decorative architectural elements and for statuary, is a challenge particularly as new and previously untested waterborne coatings are supplanting traditionally used solvent-based polymers as protective coatings. Studying film formation of coatings to better prevent corrosion of culturally significant works is an important problem in the field of conservation science. This work aims to further understanding of differences between films formed from waterborne lattices versus solvent-based polymeric coatings on bronze substrates using electrochemical impedance spectroscopy and gravimetric analysis to investigate the changes of coatings as they formed solid films. Using equivalent electric circuit models, electrochemical snapshots of the developing films were produced. Those models established time points for the transitions between theoretical stages of the nascent films and allowed for a comparison between two major classes of coating types (using the waterborne Rhoplex ${ }^{\mathrm{TM}}$ WL-81 and the solvent-based Paraloid ${ }^{\mathrm{TM}}$ B-44). Electrochemical analysis showed that diffusion occurs during early stages of film formation of both coating types. The prolonged coalescence of coatings produced from the waterborne latex was 
found to leave those films susceptible to water sorption. Annealing films as a means to drive out remaining hydrophilic additives and to facilitate film formation was investigated.

\subsection{Introduction}

There is a clear need for durable, environmentally friendly waterborne coatings that must meet or exceed the performance of current solvent-based systems for the conservation of high-value outdoor metalwork (such as statuary, architectural elements and bridges). ${ }^{9-14}$ The technical challenges for wide-spread use of waterborne coatings lie not only in the development of UV- and oxidation-resistant polymers, but also in producing continuous, defect-free films from the aqueous phase; because, unlike solvent-based coatings, aqueous colloidal suspensions contain a number of chemicals added into the coating formulation to aid in the application and drying process (e.g. surfactants, defoamers, coalescing agents and rheology modifiers). ${ }^{24-27}$ In addition to formulation additives, application of waterborne coatings on reactive surfaces such as metal alloys of copper and iron require the use of flash-rust inhibitors, either as a pre-

treatment or as a coating additive. ${ }^{28-31}$ The added step of anti-corrosive treatments complicates the interpretation of coating performance.

While no two solvent-based and waterborne coating systems are perfectly identical, the commercially available resins selected for study have similar chemical and physical properties and we believe they are the most directly comparable resins for studying early film formation differences for outdoor metal applications. In this study, differences in the formation of acrylic films cast from a solvent-based (dissolved 
polymer) or a waterborne (polymer emulsion) coating onto bronze substrates were explored using electrochemical impedance spectroscopy (EIS) as a form of rapid assessment of changes in the barrier properties of the films. ${ }^{20,} 93,103$ Mass loss curves during film drying were compared to EIS data and the data were used to correlate physical changes with electrochemical changes of the two film types. The electrochemical changes observed during drying were fit using circuit models and support theoretical physical changes in film structure.

\subsection{Background}

The mechanisms of film formation from solvent-based versus waterborne coatings are significantly different and have been examined through a variety of means in multiple fields ${ }^{32}$. Although intermediate phases remain a point of contention among many, Figure 3.1 depicts the generally agreed-upon theoretical stages of drying for films cast from solvent-based polymers $(A)$ and waterborne polymer emulsions (B). ${ }^{33^{-36}}$ As shown in Figure 3.1A solvent-based coatings go through two stages during drying: bulk evaporation (I) and diffusive evaporation (II). Immediately following application, the long polymer chains begin to entangle into a tight network, eventually producing a solid film as the solvent evaporates. This process results in a drying curve with a steep slope and thus the rapid loss of approximately $90 \%$ of the solvent by mass. The remaining solvent is lost over a longer period of time with evaporation slowed in stage II by the tighter polymer network formed during stage I. 
In contrast, waterborne films typically have three stages before film formation is complete..$^{32}$ During the first stage, the latex close-packs and retains a minimum volume of water plus additives of $36 \%$ (if the latex forms a perfect close-packed structure).

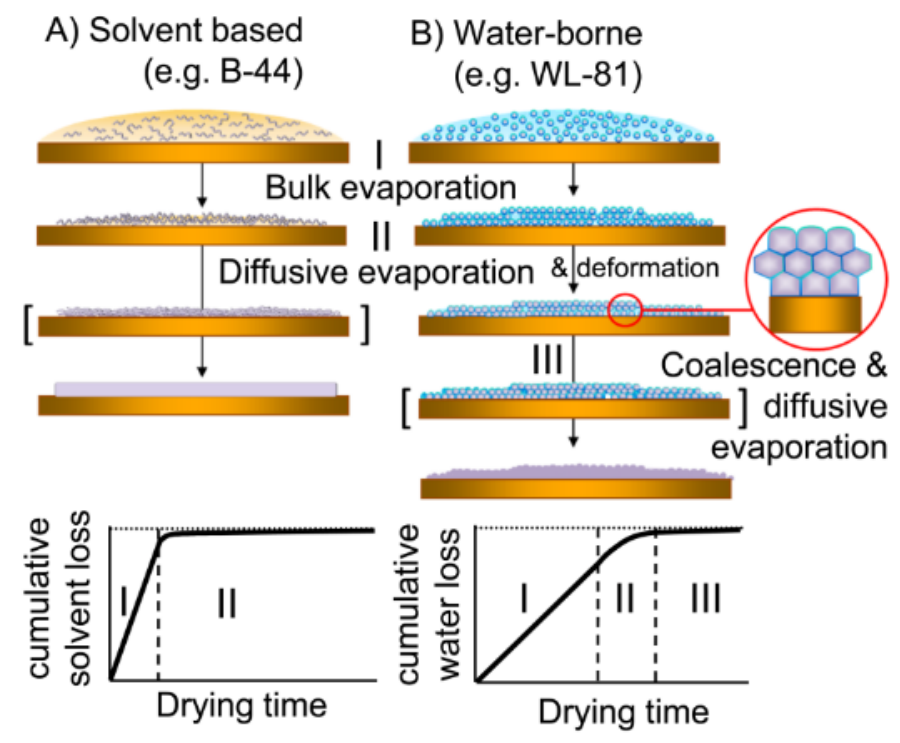

Figure 3.1 Drying time schematic of latex coating systems A) solvent-based (polymer dissolved in solvent) and B) waterborne (latex particles dispersed in water). Typical solvent-based films contain two drying stages while waterborne films progress through three stages. Figure adapted from Le Pen et al. ${ }^{33}$

During the second and third stages the water and additives occupying the free space between spheres are forced out by capillary pressure, aided by coalescing agents that help to temporarily plasticize the latex, allowing it to deform so that the additives and residual water may evaporate from the film and complete the formation of a continuous, tightly packed polymer film. ${ }^{18,104-107}$ The coalescing agents typically used in waterborne polymer emulsions have low vapor pressures (e.g. 2-butoxyethanol, vapor pressure $=79.9 \mathrm{~Pa}$ at $20^{\circ} \mathrm{C}$ ) compared to organic solvents used in solvent-based coatings (e.g. toluene, vapor pressure $=2.93 \mathrm{kPa}$ at $20^{\circ} \mathrm{C}$ ), meaning they diffuse more slowly through the polymer film and are retained within the film for longer periods of time. 
The slowest step in film formation of waterborne latexes is the third stage of the drying process and the exact mechanism of drying front propagation through the coating has been extensively studied. ${ }^{108-109}$ We believe the differences in film formation between solvent and water based polymer films, e.g. slower drying times, are critical for understanding performance differences in current coatings technology.

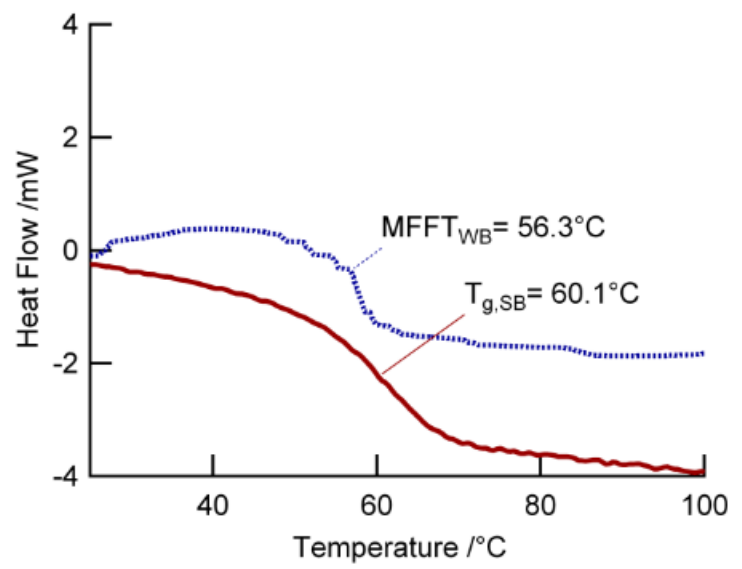

Figure 3.2 Differential scanning calorimetry thermograms of waterborne and solvent-based polymers, with an MFFT of $56.3^{\circ} \mathrm{C}$ and a $\mathrm{Tg}$ of $60.1^{\circ} \mathrm{C}$, respectively.

Latex polymer resins selected were of comparable chemistry (both copolymers of acrylic acids); both are thermoplastic coatings and have similar glass transition temperature $\left(T_{g}\right)$ or minimum film formation temperature (MFFT) so that fundamental principles of physical processes as opposed to chemical composition were the focus of analysis. Differential scanning calorimetry (DSC) was performed on both polymers to verify their Tg or MFFT and results in Figure 3.2 , indicated $a T_{g}$ at $60.1^{\circ} \mathrm{C}$ for the solventbased coating and a similar process in the waterborne coating with a MFFT at $56.3^{\circ} \mathrm{C}$. To further facilitate comparison between the two waterborne and solvent-based coatings, most additives were left out of the formulation because they can alter both the short and long-term performance characteristics of the dry films as well as 
complicate our data interpretation. ${ }^{23,104,110}$ While the physical model of film formation is well known, the electrochemical changes associated with these films' barrier properties that accompany the drying phases have not been previously described.

\subsection{Experimental}

\subsubsection{Materials}

Waterborne coatings were formulated with Rhoplex ${ }^{\mathrm{TM}}$ WL-81 (a copolymer resin composed of mostly methyl methacrylate, butyl acrylate, and methacrylic acid with some styrene, Dow, Inc.) and water (solvent); with pre-mixed 2-butoxy ethanol (coalescing agent, Acros Organics), 2-(2-butoxyethoxy) ethanol (coalescing agent, Acros Organics), BYK-301 (rheology modifier, BYK, Inc.), and Patcote 519 (defoamer, Hydrite Chemical Co.) added to the polymer/water emulsion after it was modified to a $\mathrm{pH}$ of 8.9 with dilute ammonium hydroxide. Solvent-based coatings were formulated with Paraloid ${ }^{\mathrm{TM}}$ B-44 (binary copolymer resin of ethyl acrylate/methyl methacrylate, Dow, Inc.) dissolved in toluene (solvent, Sigma Aldrich). Substrates used in the mass loss experiments were black vinyl scrub test panels $\left(\mathrm{P}_{121-10 \mathrm{~N}}\right.$, Leneta, Inc., cut into $5 \times 5 \mathrm{~cm}$ strips) and bronze coupons were used in the EIS studies $(8 \times 15 \mathrm{~cm}, 85 \% \mathrm{Cu}, 15 \% \mathrm{Sn}$ 85/15 CDA910 Tin Bronze, TB Hagstoz \& Son Inc.). Bronze substrates were degreased with p-xylene and acetone, sanded to create a uniform surface using 400 and 600 grit sandpaper, and treated to prevent flash rust by immersion in an ethanol solution of 17 $\mathrm{mM} 1 \mathrm{H}$-benzotriazole (BTA, Alfa Aesar, Inc.) at $25^{\circ} \mathrm{C}$ for 1,12 or 24 hours. Sodium 
Chloride ( $\mathrm{NaCl}$, Fisher Scientific) was used to make a $3 \% \mathrm{NaCl}\left(\mathrm{w} / \mathrm{w}\right.$ in deionized $\mathrm{H}_{2} \mathrm{O}$ ) solution with a calculated resistivity of $15 \cdot 4 \Omega \cdot \mathrm{cm}$.

\subsubsection{Electrochemical Impedance Spectroscopy}

Coatings were applied to dry, treated bronze substrates by spraying with a Fuji HVLP Super 4 XPC ${ }^{\mathrm{TM}}$ at a dry film thickness of $30 \mu \mathrm{m}$ for film formation analysis during drying and $35 \mu \mathrm{m}$ for gravimetric studies. Measurements were collected with Gamry Reference 600 Potentiostat and Gamry Framework software. EIS measurements were performed with a standard three-electrode Gamry Paint cell set up: a glass cell filled with $3 \% \mathrm{NaCl}$ was clamped to a coated bronze panel acting as the working electrode. A saturated calomel reference electrode and graphite counter electrode were immersed in the electrode solution with a $4 \mathrm{~cm}$ distance between the counter and working electrodes. An area of exposure of the working electrode equal to $14.6 \mathrm{~cm}^{2}$ was used for analysis from $1 \mathrm{MHz}$ to $0.01 \mathrm{~Hz}$ with $\mathrm{AC}$ voltage $20 \mathrm{mV}$ rms, and $\mathrm{DC}$ voltage $0.0 \mathrm{~V}$ vs. open circuit potential $-200 \pm 80 \mathrm{mV}$. Spectra were interpreted and modeled against equivalent electrical circuits (EECs) with Gamry Echem Analyst using the Simplex method. Errors in fit EEC models to the data were calculated with Echem Analyst as the sum of residual

error where the fit differed from the experimental data. Average potentiostat instrument error ranged from $3 \%$ at low frequencies to less than $0.5 \%$ at high frequencies.

During soaking EIS measurements reported were the average of three subsequent trials spanning 30 minutes and errors reported were one standard deviation 
from the mean. The relative change in total impedance of the solvent-based film at $0.1 \mathrm{~Hz}$ varied over $1.56 \pm 2.76 \%$ during the 36 hour soak, and similarly the total impedance of the solvent-based coating during a second soak varied within $3.23 \pm 3.31 \%$ of the starting value.

\subsubsection{Gravimetric analysis}

Waterborne and solvent-based coatings were applied to the black Leneta charts with an adjustable micrometer film applicator at an appropriate wet thickness to produce a dry film thickness of $20,30,40$, or $50 \mu \mathrm{m}$, applied coatings were left to air dry for one week. Coated Leneta charts underwent a soaking/drying cycle in DI water and room temperature $\left(21^{\circ} \mathrm{C}\right.$ and $\left.36 \% \mathrm{RH}\right)$. A Mettler AE260 Deltarange analytical balance was used to record daily mass changes within $0.0001 \mathrm{~g}$, the instrument sensitivity limit. The initial mass as well as any mass change of the control vinyl (non -coated) was removed from each calculation so that only changes in the film are displayed. Calculations of percent mass change are relative to the starting mass of the film for the various soaking conditions and errors were calculated by propagating the error in initial and final measurements which was, on average, $\pm 0.0005 \mathrm{~g}$.

\subsubsection{Annealing conditions}

Bronze panels were pre-treated with BTA and coated according to the above conditions with a final film thickness of $30 \mu \mathrm{m}$. The coated substrates were left to dry for one week at $21^{\circ} \mathrm{C}$ and $36 \% \mathrm{RH}$ and annealed in an oven at $60^{\circ} \mathrm{C}$ for 16 hours. EIS was performed according to the above conditions before and after annealing. 


\subsection{EIS modeling of uncoated bronze substrates}

To aid in our understanding and interpretation of barrier properties of coated substrates, uncoated substrates treated with flash rust inhibitors were studied to establish baseline levels of electrochemical barrier properties. Bronze substrates pretreated with BTA were used as our control for uncoated panels. Although in practice flash-rust inhibitors are usually only necessary when the substrate is to be coated with a waterborne coating, in these studies, all metal substrates tested were treated with BTA for 12 hours prior to coating application to enable comparison across samples. The Bode plot of BTA-treated bronze substrates immersed for either 1,12 or 24 hours (without any additional protective coating) are shown in Figure 3.3. The data from the panel treated with BTA for 24 hours prior to measurement was fit to a simple equivalent circuit, shown in Figure 3.3B. Fitting the data to equivalent circuits allowed the extraction of meaningful values for individual circuit elements: the resistance of the BTA layer had a value of $3.8 \mathrm{k} \Omega$. A constant phase element (CPE) was used instead of an ideal capacitor in the circuit in Figure 3.3B, so as to improve the fit and accuracy of the resistance values, ${ }^{111-112}$ and had a fit value of $\mathrm{CPE}_{\mathrm{BTA}}=44.12 \times 10^{-6} \mathrm{~S} \cdot \sec ^{\alpha}, \alpha=0.858$.

Data presented above (and by others) is evidence that the BTA layer alone, with its low resistance of approximately $4 \mathrm{k} \Omega$, provides inadequate protection from corrosion over periods of prolonged exposure. ${ }^{29}$ The modeled resistor fit of the layer is similar to that of an intentionally-applied patina on bronze $\mathrm{e}^{91,113}$ and at less than $1 \mathrm{M} \Omega$ is considered poor protection against corrosion where good protection is established as a coating with resistance of at least $100 \mathrm{M} \Omega .{ }^{102} \mathrm{~A}$ lack of sufficient protection against 

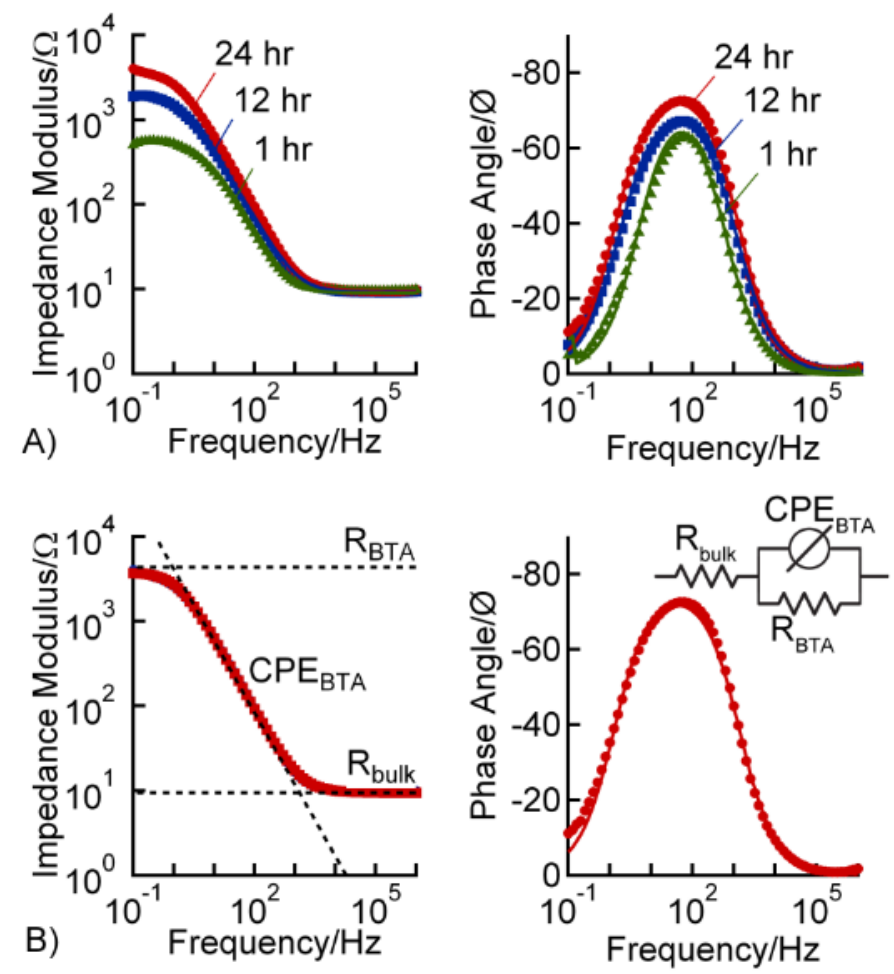

Figure 3.3 Electrical impedance spectrum of BTA layer alone after pretreatment of bronze. The markers represent experimental data and the lines indicate the modeled fit using the equivalent circuit shown. A) EIS spectra of bronze immersed in an ethanol BTA solution for 1, 12 or 24 hours. B) Modeled fit of the 24 $\mathrm{h}$ immersion BTA layer on bronze contained the following circuit elements: $R_{\text {bulk }}=9.48 \pm 0.0519 \Omega, R_{B T A}=$ $3.83 \times 10^{3} \pm 35.4 \Omega, \mathrm{CPE}_{\mathrm{BTA}}=44.12 \times 10^{-6} \pm 5.76 \times 10^{-7} \mathrm{~S} \cdot \mathrm{sec}^{\alpha}, \alpha=0.858 \pm 0.00211$. Modeled fits using the same circuit model (data not shown) of $12 \mathrm{~h}$ immersion in BTA had circuit element values of: $R_{\text {bulk }}=9.12 \pm$ $0.0508 \Omega, R_{B T A}=1.970 \times 10^{3} \pm 17.9 \Omega, C P E_{B T A}=72.18 \times 10^{-6} \pm 1.05 \times 10^{-6} \mathrm{~S} \cdot \mathrm{sec}^{\alpha}, \alpha=0.824 \pm 0.00235$, and $1 \mathrm{~h}$ immersion: $R_{\text {bulk }}=8.94 \pm 0.0511 \Omega, R_{B T A}=560 \pm 1.81 \Omega, C P E_{B T A}=92.5 \times 10^{-6} \pm 1.67 \times 10^{-6} \mathrm{~S} \cdot \mathrm{sec}^{\alpha}, \alpha=0.864 \pm$ 0.00291.

corrosion was seen by visual inspection as well: when the electrolyte cell was removed from the substrate after data acquisition, a darkened circle of corrosion products had formed on the metal surface after one hour of exposure to electrolyte solution (with analysis itself taking approximately fifteen minutes). Modeled BTA layers were used in film formation studies when applicable, although in general the resistance and/or capacitance of said layer, much like the active corrosion processes on the metal surface, did not dominate the equivalent circuit at frequencies measured and is thus considered negligible when compared to the barrier properties of the polymer coating. ${ }^{114}$ 


\subsection{EIS and equivalent circuit models during the film formation process}

Having established baseline electrochemical performance of uncoated bronze panels pre-treated with a flash rust inhibitor, the electrochemical changes associated with film formation of both a common solvent-based and waterborne coating was monitored using impedance spectroscopy. The impedance of polymer films on bronze after application of a waterborne or solvent-based coating was periodically monitored as the films progressed to dryness. Because of the rapid drying time of solvent-based films, within five minutes after coating application the coating was tacky to the touch and the EIS spectrum accompanying this stage is shown in Figure 3.4A. After an additional fifteen minutes of drying, when the film felt dry and solid to the touch, the impedance spectrum shown in Figure 3.4B was obtained. Accompanying each EIS spectrum in Figure 3.4 are the equivalent circuits that fit the film formation process of the solventbased coating.

The changes in the values of the specific circuit elements provided insight on the physical changes that accompanied transitions of the film. The EEC that provided the best fit to the data in the 'tacky' stage, Figure 3.4A, required the inclusion of a Warburg diffusion element, which commonly describes data having a phase angle of $45^{\circ}$ and physical systems exhibiting diffusion. ${ }^{115-116}$ The resistance of the solvent-based coating at its earliest time point measured by EIS, $R_{\text {coat }}$ in Figure $3.4 \mathrm{~A}$, was assigned a value of $9.25 \mathrm{k} \Omega$ based on the fit. Because of the physical state of the film: that it was tacky to the touch and that the best fit circuit model required a diffusion element, the data in3.4A likely correspond to the second phase of solvent-based film formation drawn in 

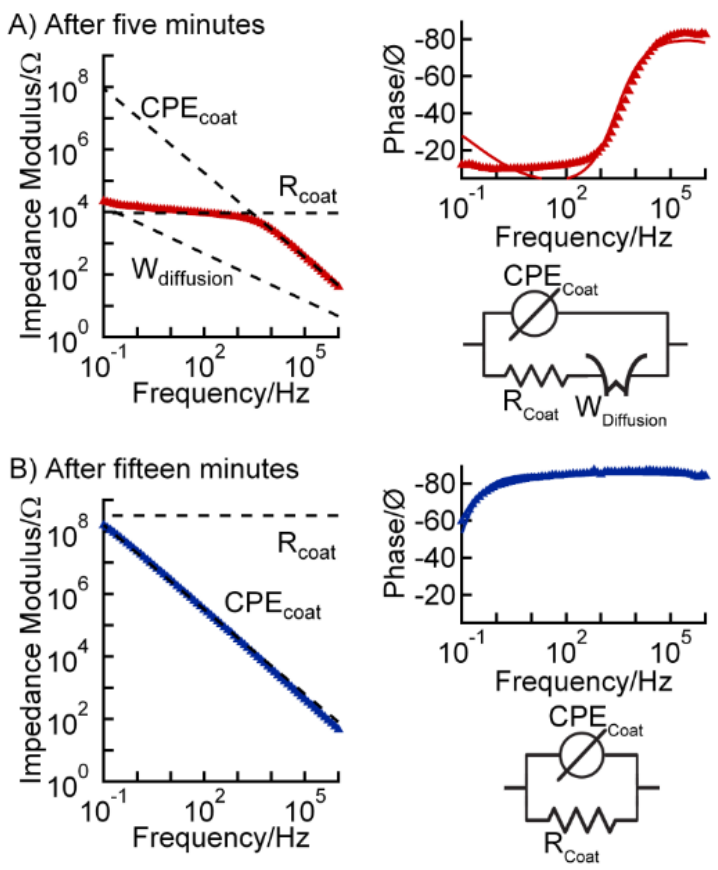

Figure 3.4 EIS Bode plots of solvent-based film formation with data fit to models with the following circuit elements: A) Tacky film (5 min) $R_{\text {coat }}=9.25 \times 103 \pm 56.4 \Omega, C P E_{\text {coat }}=16.9 \times 10-9 \pm 6.20 \times 10-10$ $\mathrm{S} \cdot \sec \alpha, \alpha=0.891 \pm 0.00276 ; \mathrm{B})$ Dry film (15 min): $\mathrm{R}_{\text {coat }}=3.18 \times 108 \pm 8.72 \times 106 \Omega, \mathrm{CPE}_{\text {coat }}=7.43 \times 10-9 \pm$ $4.63 \times 10-11 \mathrm{~S} \cdot \sec \alpha, \alpha=0.946 \pm 0.0068$.

Figure 3.1 which corresponds to the diffusive evaporation of solvent through entangled polymer chains. It is possible that the electrolyte solution slowed drying of the solventbased film, as the components of that formulation are not soluble in water, which allowed the electrochemical properties of the tacky film to be measured though the film was left in a non-ideal state for electrochemical analysis. ${ }^{117}$ After 15 minutes of drying at room temperature a dry film resistance of $0.318 \mathrm{G} \Omega$ was achieved in Figure 3.4B, an increase of five orders of magnitude over the previous value. The capacitance of the solvent-based coating was modeled with a $\mathrm{CPE}_{i}$ but because the exponent parameters of the CPE in Figure 3.4 were equal to 0.9 or higher, an ideal capacitor, which has an exponent parameter equal to 1.0 may be substituted to provide values with more meaningful units. Capacitance values were calculated at $0.1 \mathrm{~Hz}$ from the CPE 
models in Figure 3.4 and the capacitance of the film was shown to decrease as the bulk solvent evaporated. An initial calculated $\mathrm{C}_{\text {coat@o.1Hz }}$ equal to $17 \mathrm{nF}$ in Phase II (Figure 3.4A) decreased to a final dry film capacitance of $7.6 \mathrm{nF}$ (Figure 3.4C). (EIS data taken between those shown in 3.4A and 3.4B show intermediate values of capacitors.) The product of the coating's resistance and capacitance at a phase angle of $45^{\circ}$, the RC time constant, shifted to lower frequencies during drying. A physical rationale for the longer time constant observed during drying is provided by conceptualizing the slower movement and decreased number of ions in the coating as the film became solid. As described in Figure 3.1, waterborne coatings require an extra step (coalescence) in their film formation compared to their solvent-based counterparts. The three phase drying processes of a waterborne film on bronze and its final dry film stage are detailed in Figure 3.5. Spectra were obtained over a two-hour period.

The equivalent circuit of the initial tacky film is shown in Figure 3.5A, which was taken 30 minutes after coating application. Three resistors were required to fit the data, which represent the bulk solution, the coating and the BTA layer. The bulk solution resistance was easily assigned to its known value of approximately $9 \Omega$. It is interesting to note that the resistance of the bulk solution never dominated the circuit in the solvent-based film formation data in Figure 3.4 (because the resistance of the coating itself, even at the 5 minute tacky stage, was greater than the bulk solution), but the resistance of the bulk solution was observed previously in the BTA molecular layer on bronze in Figure 3.3. The resistance of the coating in Figure 3.5A had a value of $68.8 \mathrm{k} \Omega$, while the resistance of the BTA layer was $0.836 \mathrm{k} \Omega$. Two constant phase elements were 

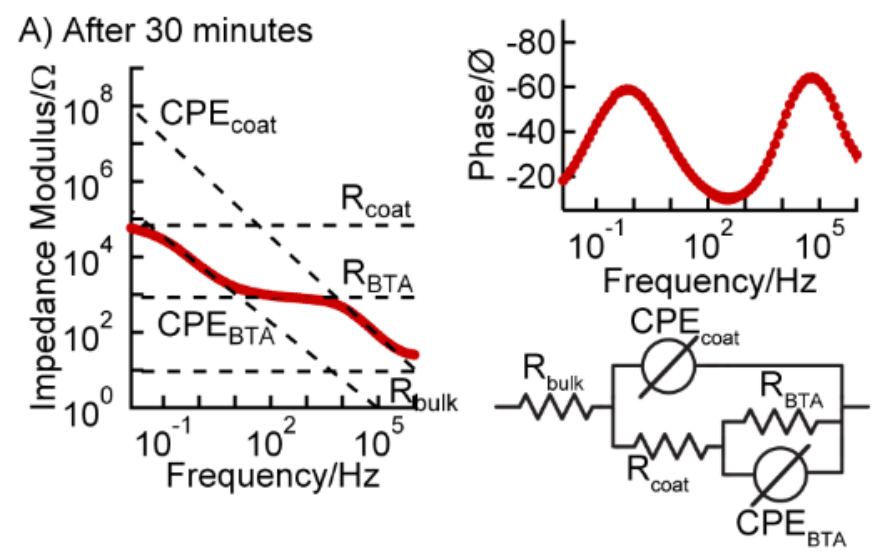

B) After 60 minutes
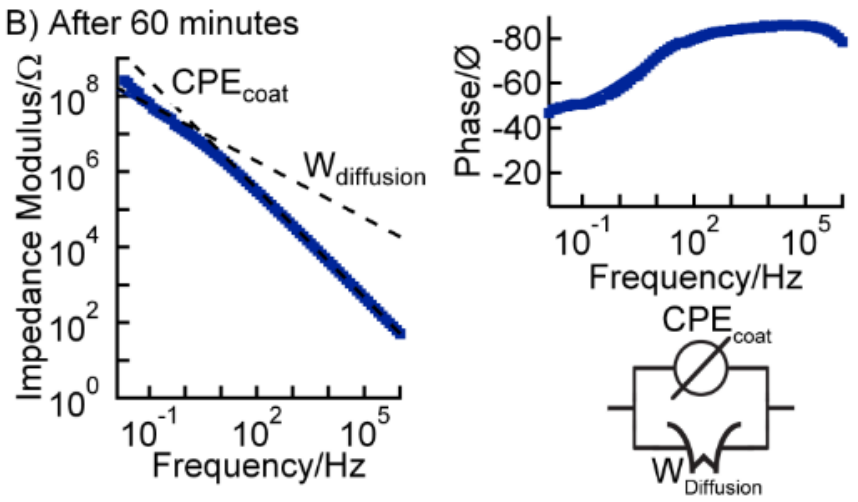

C) After 120 minutes
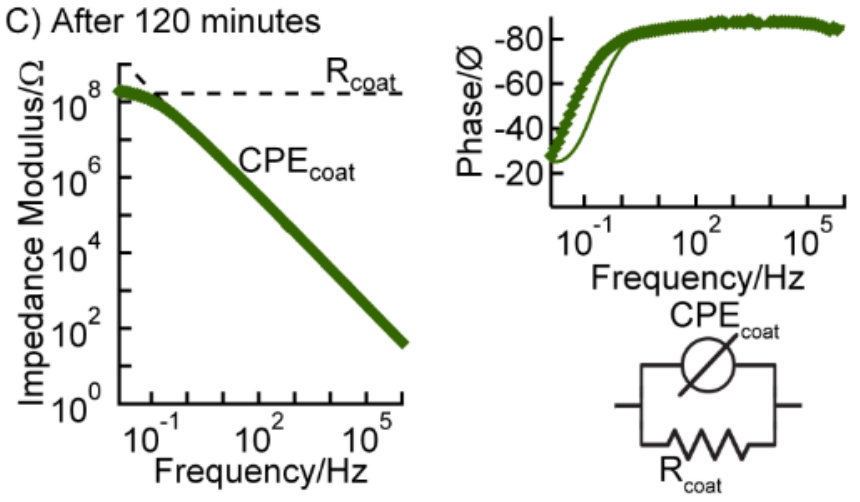

Figure 3.5 EIS Bode plot of waterborne film formation with data fit to models with the following circuit elements: A) Tacky film (after $30 \mathrm{~min}, R_{\text {bulk }}=9.13 \pm 0.458 \Omega, R_{\text {coat }}=6.88 \times 10^{4} \pm 1.29 \times 10^{3} \Omega, C P E_{\text {coat }}=106.4 \mathrm{X}$ $10^{-9} \pm 7.14 \times 10^{-9} \mathrm{~S} \cdot \mathrm{sec}^{\alpha}, \alpha=0.874 \pm 0.00529, R_{\mathrm{BTA}}=836 \pm 7.47 \Omega, \mathrm{CPE}_{\mathrm{BTA}}=41.12 \times 10^{-6} \pm 4.41 \times 10^{-7} \mathrm{~S} \cdot \mathrm{sec}^{\alpha}, \alpha$ $=0.759 \pm 0.00401 ;$ B) Semi-dry film (after 6o min) $C^{2} E_{\text {coat }}=5.87 \times 10^{-9} \pm 7.24 \times 10^{-11} \mathrm{~S}^{-\sec ^{\alpha}}, \alpha=0.959 \pm$ $0.00114, W_{\text {diffusion }}=21.1 \times 10^{-9} \pm 1.70 \times 10^{-10} ;$ C) Completely dry film (after 120 minutes), $R_{\text {coat }}=1.69 \times 10^{8} \pm$ $1.49 \times 10^{6} \Omega, C P E_{\text {coat }}=7.55 \times 10^{-9} \pm 4.74 \times 10^{-11} \mathrm{~S} \cdot \sec ^{\alpha}, \alpha=0.948 \pm 0.00670$.

in the circuit model and were assigned to the BTA layer and the coating itself. After 60 minutes of drying, when the film felt dry to the touch, EIS data were again taken and produced the spectrum seen in Figure $3.5 \mathrm{~B}$. That data was fit with a single CPE and a 
diffusion constant, $W_{\text {diffusion }}=21.19 \times 10^{-9} \mathrm{~S} \cdot \mathrm{sec}^{1 / 2}$. The film formation model in Figure 3.1 shows that coalescing agents remain within the coating after the first stage of film formation and must diffuse out of the coating in the subsequent stages. Our data taken 60 minutes after application suggests that this time point corresponds to the second phase of waterborne film formation because diffusion was indeed observed, as indicated by the inclusion of the Warburg diffusion element in the circuit model. While the film felt dry to the touch after 60 minutes, the EIS data show that the barrier properties of the film at that stage were relatively poor, particularly when compared to the data obtained after the film had dried for 120 minutes (data shown in Figure $3.5 \mathrm{C}$ ). The completely dry film shown in Figure $3.5 \mathrm{C}$ had a final coating resistance equal to 170 $\mathrm{M} \Omega$ and constant phase element associated with the coating itself equal to $7.55 \times 10^{-9}$ S. $\sec ^{\alpha}$, which were close in value to the resistance and CPE values of the solvent-based coating in its dry film stage. The equivalent circuit models supplied here are not unique to these systems and are used in practice to describe a number of polymers in different stages of their lifetime, including degraded films, ${ }^{67}$ suggesting that partially formed films have similar barrier properties and electrochemical signatures as aged or weathered films.

While the data is not shown here, aging studies in our lab and others ${ }^{38-39}$ have shown an initial increase in barrier properties after films have dried for thirty days and longer, which is interpreted as continued improvement in protection by films produced from waterborne coatings over the initial dry film obtained after two hours drying. (However, with prolonged aging, the polymer chains break down and the barrier 
properties of the films decrease. ${ }^{40-42}$ The same phenomenon, films that have dried for some weeks show an improvement in their barrier properties, has not been observed with solvent-based films. Since we only observe this change upon extended drying in waterborne films, we attribute those latent increases in impedance to trapped additives that slowly diffused out of the film over a period of weeks.

\subsubsection{Water uptake in films of various thicknesses}

The barrier properties as monitored by electrochemical impedance spectroscopy showed that the ability of the films to resist uptake of electrolyte changed during film formation and drying. After 120 minutes of air drying the films exhibited excellent barrier properties as measured by EIS, during which time films were immersed in electrolyte for approximately twenty minutes. To further understand the relationship between barrier properties of films, coatings of varying thickness that had been allowed to air-dry for one week were immersed in water and their mass changes measured over time. Water uptake into solvent-based and waterborne films as a function of dry film thickness (from 20-50 $\mathrm{mm}$ ) is depicted in Figure 3.6. In all films the largest increase in water uptake was seen during the first day of soaking. The solvent-based films of varying thickness did not exhibit significant variation in the amount of water absorbed as a function of thickness, but did absorb approximately $5 \%$ of their dry mass after a couple of days of immersion in water and did not appreciably absorb more water for the remainder (Figure 3.6A). 


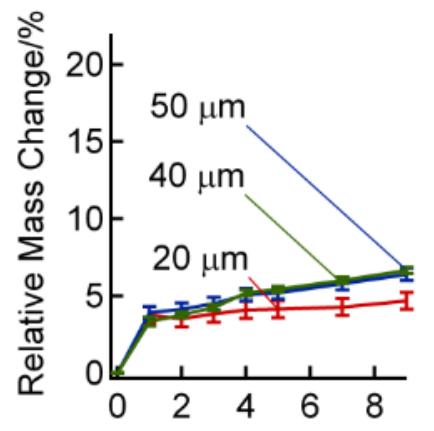

A) Time Soaking/days

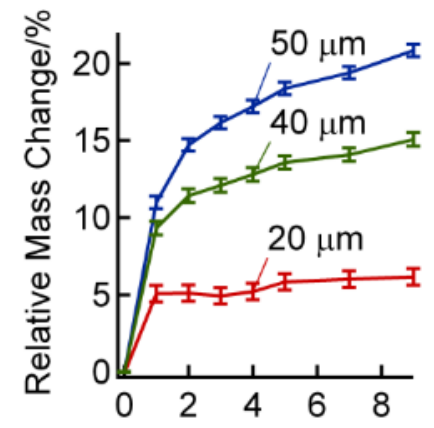

B) Time Soaking/days

Figure 3.6 Water uptake depicted by relative percent mass change to original mass of A) solvent-based and B) waterborne films of increasing thickness over a nine day soak.

In Figure 3.6B, we observed that the waterborne films absorbed more water than the solvent-based films of the same thickness did. The thicker films from waterborne coatings absorbed more water than thinner films did and the thickest of waterborne films did not saturate after 9 days of immersion (taking into account the error of the measurement). In both films, two distinct phases of water sorption occurred: approximately half of the total relative mass increase occurred in the first 24 hours and the rest over the following eight days. The average of the slope over the first 24 hours for the waterborne coating was $8.50 \%$ mass increase per day, while that for the solvent-based was $3.69 \%$ per day. After the first 24 hours, the rate of water uptake decreased, which was reflected in flatter slopes: $0.629 \%$ mass increase per day for the last 8 days of the waterborne coating and $0.287 \%$ mass increase per day for the solventbased. Since water can ingress into the film through multiple pathways, a few explanations have been previously proposed for similar uptake rates as seen in our results. ${ }^{68,118}$ For example, Cao-Paz et al. argued that the initial uptake in their films under study (11 mm films of an organic coating for automotive applications) occurred 
rapidly (with $\sim 50 \%$ total water mass uptake over the course of one hour) and that initial uptake was due to filling pores and voids at the surface. ${ }^{118}$ They further observed that the remaining mass uptake occurred more slowly (over 1 hour) through saturation of pores and voids in the bulk coating. Our observations in Figure 3.6 are consistent with that proposed mechanism: rapid initial uptake of more than half the total water mass and a gradual increase in mass until the coating was saturated. Our data are consistent with results of others ${ }^{119-122}$ and suggest that films requiring multiple applications of the coating to build up the requisite thickness adsorb water more slowly due to heterogeneous pore and/or void structures in the different layers and a greater mass of water is absorbed with increasing film thickness.

In an attempt to correlate gravimetric data with physical changes in film structure, concurrent EIS studies were undertaken. Spectra were acquired from films produced from solvent-based and waterborne coatings, which after drying, were immersed in electrolyte over a period of 36 hours. Because dry films exhibit their maximum total impedance at low frequencies (see Fig $3.4 \mathrm{~B}$ and $3.5 \mathrm{C}$ ), the total impedance of the films at $0.1 \mathrm{~Hz}$ was recorded every 6 hours during immersion and that data plotted against percent mass change of films over time as shown in Figure 3.7. Two sequential data sets for each film were taken (after four days of drying between each set) because it is believed that waterborne films leach water soluble additives over time $^{123}$ and to distinguish whether the changes observed were due to uptake of water/electrolyte into voids and defects within the film, or due to the presence of hydrophilic components retained within the film. Similar to the time-course gravimetric 
data shown in Figure 3.6, the solvent-based film's impedance did not change as much as the waterborne film's did, as seen by the clustering of data points compared to the longer lines in the waterborne data detailed in Figure 3.7.

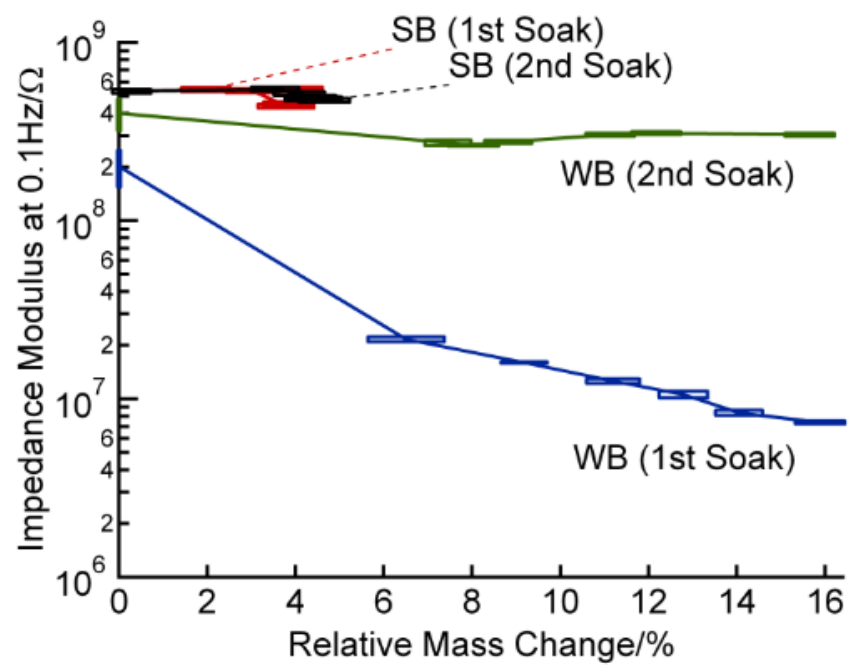

Figure 3.7 Change of total impedance at $0.1 \mathrm{~Hz}$ of solvent-based (SB) and waterborne (WB) films versus percent change in mass of equivalent films on vinyl after soaking for 36 hours. Two consecutive soaks with four days drying between are shown.

Fit values of capacitance using the ECC in Figure $3.3 \mathrm{~B}$ can be used to calculate the volume fraction of water (VF\%) in electrolyte-saturated films using the BrasherKingsbury equation. ${ }^{43}{ }^{111,124-125}$ Using that method, the waterborne film showed a VF of $6.0 \pm 0.94 \%$ after the first $36 \mathrm{~h}$ immersion and showed a VF of $2.4 \pm 0.38 \%$ after the second immersion. Because the second soaking of the waterborne film showed a significant difference electrochemically from its first soaking, that suggested that indeed, hydrophilic additives such as coalescing agents, were retained in the film after air drying. The solvent-based film acted as a control in this study (as it did not contain hydrophilic additives, unlike the waterborne film): the second soak of the solvent-based film did not result in any significant differences over the first. (For comparison, the 
solvent-based coatings showed a $-0.48 \pm 0.43 \%$ and $-0.53 \pm 0.38 \% \mathrm{VF}$ after the first and second soak, respectively, nearly insignificant changes.) Although some variation in impedance was seen during replicate EIS runs, those fluctuations were less than $3 \%$ of the total impedance, and thus are negligible within the error of the measurement; suggesting that the changes observed were, in fact, unique to the waterborne film and significant. ${ }^{111}$

Volume fration values as calculated by EIS were compared to those calculated by gravimetric means (using data extracted from points in Figure 3.7). By gravimetric analysis the VF of water absorbed by the waterborne film was $14.1 \pm 0.56 \%$ after the first immersion and $13.3 \pm 0.57 \%$ after the second. The solvent-based film had a VF of $4.73 \pm 0.54 \%$ after the first soak and $5.10 \pm 0.55 \%$ after the second $36 \mathrm{~h}$ immersion. Gravimetric analysis showed that VF\% absorbed did not change significantly for the solvent-based film after one or two immersions and only changed by a small amount in the waterborne film.

Comparing the estimates of VF via capacitance changes in the EIS data with mass changes in the gravimetric analysis, two significant differences between the VF values determined by the two methods became apparent: 1) the values were smaller when estimated using capacitance values and the Brasher-Kingsbury equation compared to those values measured by gravimetric means and 2) by gravimetric analysis, VF\% did not change significantly in sequential immersions, however it did change significantly by EIS for waterborne coatings (comparing $6.0 \pm 0.94 \mathrm{VF} \%$ after the first and $2.4 \pm 0.38 \mathrm{VF} \%$ after the second). Differences in the volume of water absorbed 
into the film during EIS versus the gravimetric analysis may be due, in part, to differences in the amount or rate of water uptake into the film. ${ }^{43}$ The vinyl substrates were completely immersed and thus allowed for percolation from the sides as well as surface absorption and consequently had two directions of water sorption: from the top and from the sides; in contrast, the bronze substrates were soaked only on the top surface with a limited area of exposure. The additional pathways into the coatings on vinyl may explain what appears to be an overestimate when compared to the volume fraction of water in the film calculated via modeled values of capacitance in both our

study and others. ${ }^{68}$ Additionally, changes in capacitance within the coating may not account for water absorbed in surface voids and may also contribute to the difference in VF\%. Regardless of the differences in the calculated VF\% values between the EIS and mass uptake experiments, the same trends exist in the data: the waterborne films were consistently more susceptible to water or electrolyte uptake than the solvent-based films.

\subsubsection{Post-Annealing film comparison of soaked films}

Because the data from the immersion studies in Figures 3.6 and 3.7 suggested that water soluble components remained in the film after air drying, a study in which films were heated in an oven to drive off remaining additives was carried out. Bronze panels spray coated with single layers of waterborne or solvent-based coatings were either airdried or oven-annealed and analyzed by EIS after the panels had been soaked in water for four hours (data shown in Figure 3.8). 

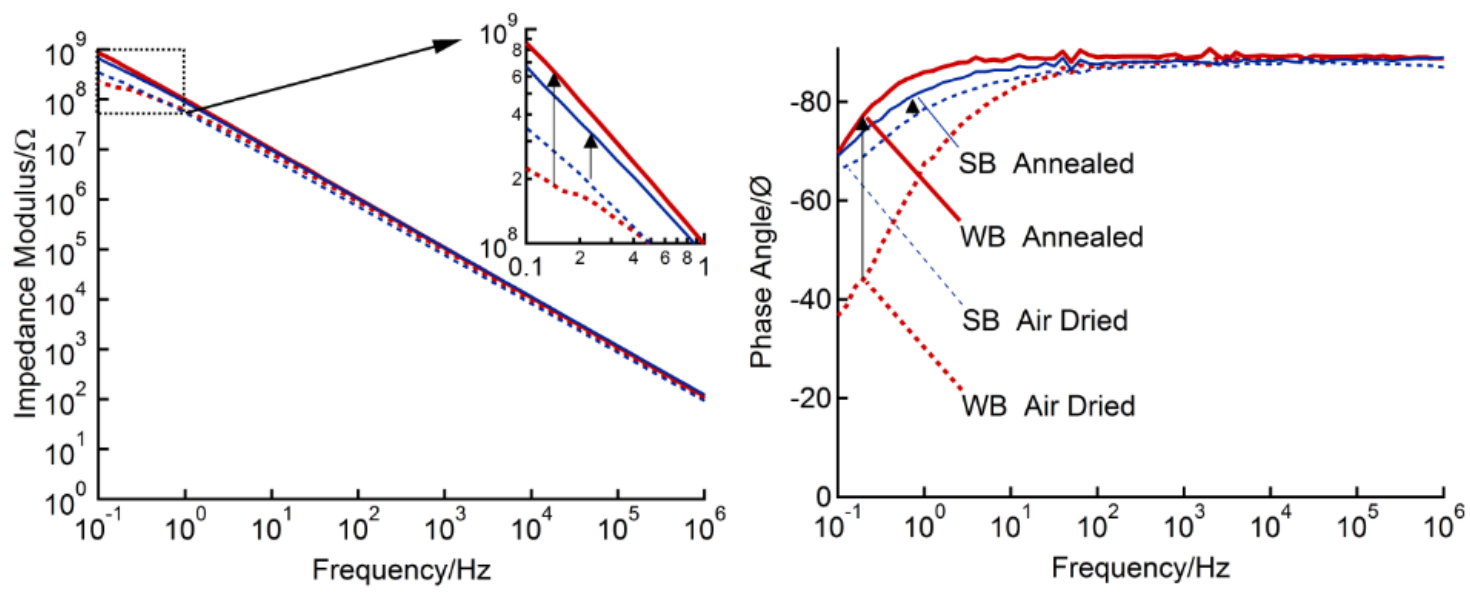

Figure 3.8 Bode plot of impedance changes of solvent-based and waterborne films. Traces are shown after a 4 hour water immersion with or without annealing in an oven at $60^{\circ} \mathrm{C}$, arrows indicate that after annealing, both film types showed an increase in total impedance and a shift in the phase angle.

After soaking, the air-dried solvent-based and waterborne coatings had total impedances at $0.1 \mathrm{~Hz}$ of 350 and $220 \mathrm{M} \Omega$, respectively, and when annealed the total impedance of both coatings approached a value of $10^{9} \Omega$, more than three times the starting value. Further insight may be gained by examining the phase angle: the frequency at which the phase equals $-45^{\circ}$ (specifically the RC time constant where $\tau_{\text {coat }}$ $\left.=\mathrm{R}_{\text {coat }} \times \mathrm{C}_{\text {coat }}\right)$ differs for each trace shown in Figure 3.8 and while only one trace actually crossed $-45^{\circ}$ in the frequency range of these data sets, circuit modeling allowed us to predict the time constant. The post-soak RC time constant for the solvent-based coating was calculated to be $1.08 \mathrm{sec}$ and increased to $2.03 \mathrm{sec}$ upon annealing. The difference in the RC time constant helps develop a conceptual picture by establishing the rate at which ions travel from the counter to working electrode through the coating. ${ }^{45}$ The RC time constant for the waterborne film before annealing (after air dying) was the fastest at $0.335 \mathrm{sec}$ and increased ten-fold to $3.33 \mathrm{sec}$ upon annealing. 
These data show the improvement in the barrier properties of both coating types that may be gained by annealing, which we attribute to improved film structure via three factors: healing of defects, completion of coalescence, and enhanced evaporation of volatile hydrophilic additives. The improvements are more dramatic in the case of waterborne films because all three factors are operant, while only one (healing of defects) is present when solvent-based films are annealed.

Theories have been supplied that describe a top layer (or skin) that dries quickly in latex films which can slow down the diffusion of coalescing agents out of the coating. ${ }^{126}$ That theory may be reinforced by our observations that the resistance of films continues to increase after thirty days of drying (data not shown). It is possible that while less than $1 \%$ of latex emulsion additives may remain in the dried film (as the mass of the films does not change appreciably during prolonged drying), if the drying front propagates down from the surface of the film towards the substrate, annealing may be a convenient means to accelerate the formation of waterborne films through stage three, by softening the polymer network and increasing the rate of coalescence and evaporation of hydrophilic components.

\subsection{Conclusion}

Our data shows that changes in the barrier properties of films during formation are associated with their coating type: whether the nascent film was produced from a solvent-based and waterborne coating. Distinct circuit models provide best fits for each data set, suggesting that indeed a different polymer network structure existed for each 
data set. In the case of the solvent-based coating, the improvements in its barrier properties post-annealing are likely due to the fact that the annealing temperature was close to the glass transition temperature of the polymer, which when thus softened could heal sub-microscopic film defects. Because the improvement in the barrier properties of the waterborne coating exceeded that of the solvent-based one, two scenarios that explain the changes observed are possible. First, the waterborne film may not have undergone complete coalescence. Support for the first scenario shown in our data includes: (a) the film's total impedance at $0.1 \mathrm{~Hz}$ was lower than that of the solvent-based coating and (b) annealing completed coalescence because the annealing temperature exceeded the polymer's minimum film formation temperature. Second, residual volatile additives remained in the film produced from the waterborne coating that were evaporated off by annealing. We do not propose that the differences observed are due (entirely) to differing amounts of microscopic film defects, like bubbles, as careful inspection of films under magnification was done to ensure that analyzed areas were defect free. A more detailed study of latent additives within the films is examined in a future publication. ${ }^{89}$ The findings in this study reinforce the established theoretical models of film formation for solvent-based and waterborne coatings. The equivalent circuit models assigned to the different stages of film formation may also be operant in film degradation processes. Differences in mass and volume changes observed between EIS and gravimetric means can be explained by differences in the physical films and the number of directions from which water may absorb into the film (from the top only or also from the sides). ${ }^{43}$ The trends in our 
findings, which are similar to those suggested by others, ${ }^{111,}{ }^{114},{ }^{127}$ during soaking of films in both gravimetric and spectroscopic studies of water uptake show that: (1) water uptake occurs in multiple phases, two of which were seen here (dramatic initial uptake into surface voids followed by saturation of the bulk polymer); (2) there is greater water uptake in waterborne over solvent-based films; (3) there is a decrease in resistance and increase in capacitance of films over long water immersion times.

If waterborne coatings are to completely replace solvent-based coatings for outdoor metals applications in the future, there is a requirement to improve the postapplication film forming process as the susceptibility of waterborne coatings to uptake water/electrolyte has negative implications for films' success as protective barriers against corrosion. Unlike its solvent-based counterpart, the extensive time required for polymer deformation and coalescence leaves waterborne coatings somewhat permeable after application. In actual outdoor applications, because water uptake lowers the resistance of the film against ion migration, the need exists to limit the exposure of drying films to wet environments. 


\section{Characterizing and improving performance properties of thin solid films produced by weatherable waterborne colloidal suspensions on bronze substrates}

Adapted with permission from: Swartz, N. A.; Wood, K. A.; Clare, T. L., Characterizing and improving performance properties of thin solid films produced by weatherable waterborne colloidal suspensions on bronze substrates. Prog. Org. Coat. 2012,75 (3), 215-223.

While the development and application for transparent protective coatings for metals continues and broadens, the use of these coatings on high-value outdoor bronze objects, such as statues and architectural elements, requires extensive testing before use. Recent efforts in coatings technology have produced high-performance waterborne latex dispersions containing polyacrylics and poly(vinylidene fluoride) (PVDF) targeting extended coating lifetimes and improved UV resistance. Our studies show that a two-layer polymer film with a solvent-based primer (Paraloid ${ }^{\mathrm{TM}}$ B-44) and a high performance waterborne topcoat (Kynar Aquatec ${ }^{\mathrm{TM}}$ RC-10206) exhibits high impedance as measured by electrochemical impedance spectroscopy. Upon annealing, those films further increased in impedance, suggesting improved corrosion protection compared to unannealed films. When soaking in water, films that contained high loading levels of coalescing agent (Dipropylene glycol monomethyl ether, DPM) in the topcoat formulation resulted in a visible whitening of the basecoat and a decrease in coating resistance. Characterization of the whitened layer by FT-IR indicated the presence of coalescent in the basecoat, suggesting that coalescent migrated from the topcoat into the basecoat. Annealing studies were performed to reduce uptake and reverse or inhibit water whitening. 


\subsection{Introduction}

In the field of conservation of material cultural heritage, the selection criteria for protective coatings for outdoor metalwork (bronze sculptures and architectural elements) are significantly different than the needs of typical consumers. Conservators select coatings based on protection against corrosion, durability, transparency, reversibility (i.e. how easily the coating may be removed), and ease of application onsite. ${ }^{20-21}$ Cost of the coating is usually not considered (due to the high value of the object itself), as long as the coating satisfactorily meets the other selection criteria. Legislative restrictions have provided a motivation to reduce volatile organic component (VOC) levels in coatings and thereby limited the array of coating options for conservators as state and federal environmental regulations have positioned waterborne coatings to replace solvent-based coatings in many applications.

However, replacing solvent-based with waterborne coatings is not a trivial matter as the two coating types greatly differ in their ability to form protective films, even when the chemistry of the coatings is similar. ${ }^{37}$ Waterborne coatings are also susceptible to blistering and water-whitening. In 1954, Wheeler et al. stated the need for a compromise in aqueous polymer dispersions between stability and performance, a tension which remains to this day. ${ }^{18}$ The industrial push for widespread use of waterborne coatings, driven both by their decreased cost and reduced environmental impact, is generally at the expense of their protective barrier properties compared to traditional solvent borne coatings. ${ }^{17}$ Recently, new highly weatherable waterborne latex technology has become available, based on poly(vinylidene fluoride) (PVDF) 
hybrid dispersions. Potentially, compared to solvent-based acrylic coatings, waterborne PVDF-based coatings may offer an environmentally-friendlier alternative, with superior corrosion protection and durability under outdoor weathering. The specific aims of this study are to understand the performance characteristics on bronze substrates of a coating formulation based on one of these PVDF hybrid dispersions, as characterized by water sorption, water whitening, electrochemical impedance spectroscopy (EIS), and Fourier transform infrared spectroscopy (FTIR).

\subsection{Background}

Ideally, the polymer resins of organic coatings should be hydrophobic to prevent water sorption; however hydrophobic polymers are not normally stable as waterborne suspensions, and so require the addition of hydrophilic additives, or the modification of the polymer to include hydrophilic components, as a means of stabilizing these suspensions. ${ }^{22-23}$ The ability of coatings to prevent corrosion also depends upon the chemistry and durability of the polymer, especially its ability to resist degradation due to UV light exposure. ${ }^{42,47-48}$ Fluorinated polymer hybrid blends based on PVDF are among the most UV-resistant resin systems used today in commercial coatings for metals, with protective properties lasting in many cases for more than thirty years of outdoor exposure, as described by Wood et al. and others. ${ }^{11,47-49}$ Since their debut in the 1960s, PVDF-containing coatings have primarily been supplied as non-aqueous dispersion or solution coatings, often at high VOC levels. Recent advances have produced waterborne (latex) hybrid dispersions with high PVDF resin content and the 
performance of these coatings continues to improve. ${ }^{50-52}$ Commercial products of this type, such as KYNAR Aquatec ${ }^{\circledR}$ RC-10206, contain $70 \%$ or $50 \%$ PVDF on total resin weight, with the remainder of the resin being acrylics. ${ }^{11,47}$ These proportions are similar to PVDF:acrylic ratios used typically in traditional solvent PVDF-containing coatings. Like solvent PVDF coatings, waterborne PVDF coatings of this type have been shown to offer superior barrier properties, after exposure to UV light, as compared to solventbased $100 \%$ acrylic coatings. ${ }^{11}$

Because of their relatively inert chemistry (which confers resistance to oxidative degradation, and other components of coating durability), 100\% PVDF coatings do not provide good adhesion to metals, compared to acrylic resins such as Paraloid ${ }^{\circledR}$ B-44, a methyl methacrylate/ethyl acrylate copolymer. We circumvent this adhesion problem by using an acrylic basecoat primer (for good adhesion) combined with a waterborne PVDF-based topcoat (for good weatherability).

However, we have identified a remaining challenge with some prototype systems of this type: the clear films are susceptible to whitening upon exposure to water. This whitening phenomenon is seen only for two-coat systems (solvent acrylic primer plus waterborne PVDF-based topcoat), not for either of the coating layers applied singly. Whitening is particularly problematic in outdoor applications for both aesthetic reasons, and for possible indications of poor short and long-term performance.

The causes of whitening in organic thin film coatings have been attributed in large part to the additives of the formulations and not to the polymer resins 
themselves. ${ }^{44}$ The first to document the phenomenon of water whitening, Wheeler et al. implicated emulsifiers as the cause. They discussed possible interactions of the hydrophilic additive and the polymer network, leading to hydrophilic inclusions which swelled with water and caused increased light scattering ${ }^{18}$ Binschaedler also noted whitening and ascribed the cause in cellulose acetate films to plasticizers and sodium dodecyl sulfate present for film formation. ${ }^{128}$ Agarwal and Farris expanded the work on surfactants to a variety of acrylic-based latex blend films, and found that all formulations whitened upon immersion in water due to hydrated surfactant reservoirs that swell and scatter light, creating a "white and opaque film". ${ }^{44}$ Robeson and Hyder found increased water sorption correlating with the additive levels, in emulsion polymer cast films. ${ }^{22}$ Polymers of dissimilar chemistry were unable to diffuse and coalesce into one cohesive phase, which in turn created a percolation network at the interface where residual additives affected water transport through the films. Water uptake, and the visual whitening effect that may follow, is increased by the hydrophilic nature of waterborne coatings, and by sub-microscopic channels in the polymer film caused by the evaporation of slow cosolvents. ${ }^{45}$

When describing surfactant effects, Keddie and Routh ${ }^{46}$ detail a worst-case scenario that could create a hydrophilic pathway to the metal; they state that surfactant accumulation around particle boundaries creates hydrophilic pockets to trap water and speed water transport. The study by Agarwal and Farris also presented evidence that softer polymers were more prone to generate "islands" of surfactant molecules, when compared to rigid counterparts. Water diffusion into coatings 
produces a swollen layer above an unaffected pristine layer in the coating, where the characteristic length scale and total quantity of the absorbed water may affect electrochemical performance. ${ }^{45,}{ }^{68}$ Based on these findings it is likely that for two-coat systems, there could be interactive effects on the system's water sorption behavior, resulting from the use of different types of polymer in the basecoat and topcoat. For this purpose in situ electrochemical spectroscopy is ideally suited to differentiate active processes that change the barrier properties of the base and topcoat. ${ }^{69}$

In our studies described below, solvent-based acrylic primer coats (of various types) and latex Kynar Aquatec topcoat were applied to metal coupons of bronze. We hypothesize that the whitening, which can be localized by optical microscopy as occurring in the top part of the basecoat layer, is caused when the coalescent in the waterborne PVDF-containing topcoat migrates into the acrylic basecoat and is trapped there after the topcoat dries. This trapped solvent plasticizes the hydrophilic acrylic basecoat and increases its permeability to water. When the coated coupons are immersed in water, coalescent-rich pockets within the acrylic layer can preferentially take up water, and when these reach the appropriate size to scatter light they produce white or opaque coatings.

\subsection{Experimental}

To better understand the complete basecoat/topcoat system's susceptibility to water whitenening, both individual layers were characterized by Electrochemical Impedance Spectroscopy (EIS), in an effort to probe whether there were defects leading to low 
impedance that could be related to water uptake and whitening. EIS measurements were performed using bronze coupons layered with various coatings and inter-layer electrodes, represented in Figure 4.1. The electrochemical analysis of individual layers was performed before and after annealing, using several annealing methods. The annealing of organic coating systems has demonstrated an improvement in mechanical properties of the films. ${ }^{129}$ While annealing in an oven is the ideal and most effective solution, alternative, more practical techniques for use in the field and outdoors, such as an applied heat gun and infrared lamp exposure, were explored.

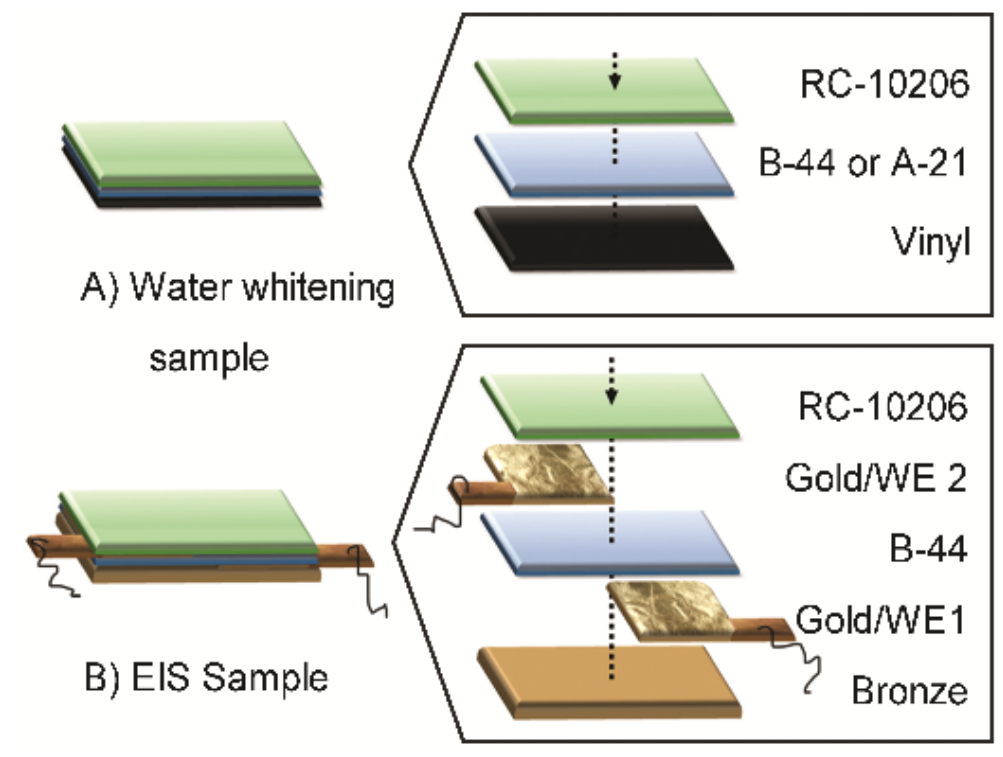

Figure 4.1 Exploded view of coating layers on substrates. A) Black vinyl scrub chart spray coated with a solvent-based primer coat and Kynar Aquatec RC-10206 topcoat. B) Bronze panel layered with gold foil and attached working electrode 1 (WE1), a primer of B-44, gold foil with attached working electrode 2 (WE2), and topcoat of RC-10206.

\subsubsection{Materials}

The bronze substrates were treated to prevent flash rust by immersing them for 24 hours in 17mM benzotriazole (Alfa-Aesar) in ethanol. Basecoats were formulated with Paraloid ${ }^{\circledR}$ B-44, Paraloid ${ }^{\circledR}$ A-21, or blends of these two acrylic resins dissolved in 
toluene. Both resins were graciously supplied by Dow, Inc. The topcoat used was waterborne Kynar Aquatec ${ }^{\circledR} \mathrm{RC}-10206$ latex (Arkema, Inc.), having a minimum film formation temperature of $28^{\circ} \mathrm{C}$, coalesced with $5 \%, 10 \%$, or $20 \%$ (w\%/w polymer) 2 -( 2 methoxypropoxy)propan-1-ol (DOWANOL ${ }^{\circledR}$ DPM, Dow, Inc.). Substrates were Leneta black vinyl scrub test panels ( $\left(P_{121-10 N}\right.$, cut into $5 \mathrm{~cm} \times 7.5 \mathrm{~cm}$ strips) and bronze coupons ( $8 \times 15 \mathrm{~cm}, 85 \% \mathrm{Cu}, 15 \% \mathrm{Sn}$ CDA910 Tin Bronze, TB Hagstoz \& Son Inc.). Gold leaf (Goldbeater's Gusto Manetti, 23k yellow gold) and Copper/Nickel tape (16067, Ted Pella, Inc). Sodium Chloride ( $\mathrm{NaCl}$, Fisher Scientific) was used to make a $3 \% \mathrm{NaCl}(\mathrm{w} / \mathrm{w}$ in distilled $\mathrm{H}_{2} \mathrm{O}$ ) electrolyte for the EIS measurements with a calculated resistivity of $15.4 \Omega$

\subsubsection{Water whitening and Mass uptake}

A basecoat of B-44 or A-21 was applied to the black vinyl charts using a Gardco adjustable micrometer film applicator at an appropriate wet thickness to produce a dry film thickness of $10 \mu \mathrm{m}$. After drying for one day, a topcoat of RC-10206 was applied to all charts to produce a dry film thickness of $15 \mu \mathrm{m}$ (total coating thickness of $25 \mu \mathrm{m}$ ). The standard formulation for the RC-10206 clear coat contained 10\% DPM and was used as the baseline for whitening in this study. A schematic of coated substrates can be seen in Figure 4.1A. Applied coatings were left to air dry for one week at $21^{\circ} \mathrm{C}$ and $36 \% \mathrm{RH}$ or annealed at $60{ }^{\circ} \mathrm{C}$ for six hours with a $1300 \mathrm{~W}$ Master heat gun, four hours with a 1500 W Infratech IR lamp, or one or sixteen hours in a $800 \mathrm{~W}$ Lab-Line Instruments, Inc. oven. 
Coated Leneta charts underwent a soaking/drying cycle in DI water at room temperature $\left(21^{\circ} \mathrm{C}\right.$ and $\left.36 \% \mathrm{RH}\right)$. A Mettler AE260 Deltarange analytical balance was used to record daily mass changes within $0.0001 \mathrm{~g}$, the instrument sensitivity limit. The initial mass as well as any mass change of the control vinyl (non -coated) was removed from each calculation so that only changes in the film are displayed. Calculations of percent mass change are relative to the starting mass of the film for the various soaking conditions and errors were calculated by propagating the error in initial and final measurements which was, on average, \pm 0.00059 . Changes in grayscale values were recorded with a Nikon $\mathrm{D}_{40}$ and images were converted to grayscale in Adobe Photoshop $\mathrm{CS}_{4}$, where the average $k$ value of the chart was obtained. Samples were illuminated according to ASTM D1729-96.

\subsubsection{Coating bronze with gold electrodes for EIS}

The working electrode ( $\mathrm{WE}_{1}$ ) was made with a $5 \mathrm{~cm} \times 5 \mathrm{~cm}$ square of gold leaf pressed onto the bronze panel. Electrical contact was made by attaching a piece of copper tape to the gold for the potentiostat clip. Substrates were spray-coated with a Fuji HVLP Super $4 \mathrm{XPC}^{\mathrm{TM}}$ for a dry film thickness of approximately $12 \mu \mathrm{m}$ for each layer. An interlayer gold electrode (WE2) was pressed on the B-44 coated panel before a topcoat of RC-10206 was applied. A schematic of the layered coatings and gold electrodes can be seen in Figure 4.1B. Control sets of bronze panels with single layers of B-44 or RC10206 were also produced. The panels were dried at $21^{\circ} \mathrm{C}$ and $36 \% \mathrm{RH}$ for one week or annealed in an oven for 16 hours at $60^{\circ} \mathrm{C}$. 


\subsubsection{Electrical impedance spectroscopy (EIS)}

Measurements were collected with a Gamry Reference 600 Potentiostat and Gamry Framework software. EIS measurements were performed with a standard threeelectrode cell set up in $3 \% \mathrm{NaCl}$ with a SCE reference electrode, graphite counter electrode and the fabricated coated bronze/gold working electrodes (see Figure 4.1B). PortHole Electroplating Tape (Gamry Part\#990-00139) was used to expose a $1 \mathrm{~cm}^{2}$ area for analysis from $1 \mathrm{MHz}$ to $0.1 \mathrm{~Hz}$ with $\mathrm{AC}$ voltage $20 \mathrm{mV} \mathrm{rms}$, and $\mathrm{DC}$ voltage o $\mathrm{V}$ relative to the open circuit potential. Spectra were interpreted and fit using equivalent electrical circuit (EEC) models with Gamry Echem Analyst and the simplex method.

\subsubsection{Infrared Microanalysis}

Characterization via $\mu$-FT-IR was performed on cross-sections of polymer films rolled onto a diamond slide. A ThermoScientific iS 10 infrared spectrometer with a Nicolet Continu $\mu \mathrm{m}$ FT-IR microscope and $50 \mu \mathrm{m} \mathrm{MCT} \mathrm{(mercury} \mathrm{telluride/cadmium} \mathrm{telluride)}$ detector was used to acquire each spectrum. Data was transformed using an N-B strong apodization function and Mertz phase correction. The spectra were acquired in transmission mode from 128 scans with $4 \mathrm{~cm}^{-1}$ resolution using Omnic software and analyzed in Ominc Spectra. A stereoscopic light microscope (Leica MZ6) was used for examination of the materials and to select areas for analysis.

\subsection{Computation Theory}

Water based coatings have an increased sensitivity to water when immersed or exposed due to hydrophilic additives that aid predominately in emulsion stabilization. 
EIS was utilized to identify changes in the permeability of films when exposed to saline solutions. Since an organic coating acts as a dielectric material sandwiched between the bulk solution and the metal, water sorption in a polymeric film typically causes an increase in coating capacitance (from an increasing dielectric constant) as well as a decrease in resistance at low frequencies. These parameters allow the volume of water absorbed into the coating to be calculated by the Brasher-Kingsbury ${ }^{124}$ equation (4.1):

$V F=\log \left(C / C_{o}\right) / \log \left(\varepsilon_{R}\right)$

where $C$ is the capacitance of the coating after water exposure, $C_{0}$ is the initial capacitance before water exposure, $\varepsilon_{\mathrm{R}}$ is the relative permittivity of water (80.1 at $\left.20^{\circ} \mathrm{C}\right)$, and $\mathrm{VF}$ is the volume fraction of water.

EIS is a valuable tool for the analysis of protective coatings because the data can be fit to circuit models, which facilitate interpretation of the system, and because it is non-invasive and non-destructive. ${ }^{112,}$ 130-132 $^{-1}$ The model for an intact film is typically described with only one time constant, i.e. one resistor and capacitor in parallel shown in Figure 4.2 Model A, and defects or film inconsistencies can be modeled with the addition of another RC pair in parallel, shown in Figure 4.2 Model B..$^{133^{-135}}$

\subsection{Results}

\subsubsection{EIS equivalent circuits used to quantify improvements in barrier properties}

To establish a benchmark for coating performance in this study, EIS spectra were collected for bronze coupons coated with only RC-10206 films (air-dried or ovenannealed). The EIS data (markers), the equivalent circuit models used in data fitting, 
Model A
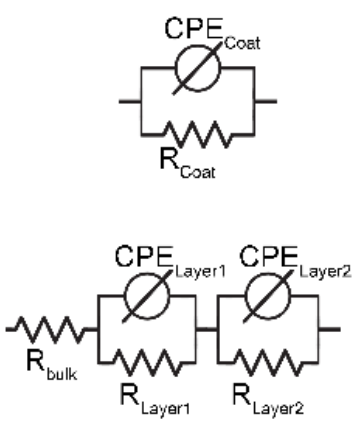

Model B
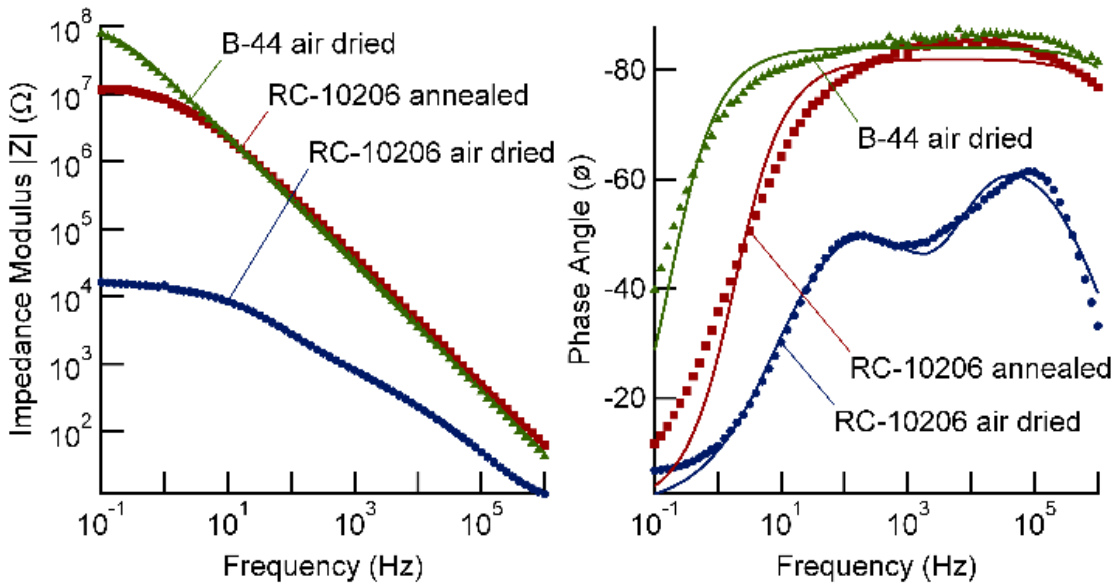

Figure 4.2 EIS of RC-10206 or B-44 coated bronze coupon and ECC models fit to experimental data. Circuit element values used in the fit of air dried $R C-10206$ using Model $B: R_{\text {bulk }}=9.1 \Omega, R_{\text {Layer2 }}=1.6 \times 10^{4} \pm$ $210 \Omega, C P E_{\text {Layer } 2}=4.58 \pm 0.81 \times 10^{-6}$ Siemens. $\sec ^{\alpha}, \alpha=0.668, R_{\text {Layer } 1}=213.5 \pm 26.1 \Omega, C P E_{\text {Layer } 1}=4.73 \pm 1.35 \times 10^{-7}$ Siemens. $\sec ^{\alpha}, \alpha=0.882 ;$ annealed $R C-10206$ using Model $A: R_{\text {coat }}=1.1 \times 10^{7} \pm 8.8 \times 10^{4} \Omega, C P E_{\text {coa }} t=9.8 \pm 0.09 \times 10^{-}$ ${ }^{9}$ Siemens $\cdot \sec ^{\alpha}, \alpha=0.91$; and air dried $B-44$ using Model $A: R_{\text {coat }}=94.8 \pm 1.42 \times 10^{6} \Omega, C P E_{\text {coat }}=9.51 \times 10$ ${ }^{9} \pm 64.1 \times 10^{-12}$ Siemens $\cdot \sec ^{\alpha}, \alpha=0.934$.

and the best fit lines (solid lines) are shown in Figure 4.2. Model B was fit to the air-dried RC-10206 coating, while Model A was fit to the annealed coating. The excellent fit of the data in the Bode plots of the "air dried" coating both confirms the theoretical circuit model for a porous film, and demonstrates that the coating under study was still susceptible to electrolyte uptake when left to dry 4 weeks at room temperature. The complex nature of activity in and around the metal substrate coated with an intact film is demonstrated by the resistance of the coating $\left(R_{\text {coat }}\right)$ and the capacitance of the coating $\left(C_{\text {coat }}\right)$. In some cases, electrolyte may diffuse into the polymer network at the coating - water interface, where the electrochemistry of the electrolyte-swollen portion and the non-swollen portion are different and the two distinct features are observable in a Bode plot ${ }^{68}$. The two layers consist of a water saturated portion of the coating represented by $\mathrm{R}_{\text {Layer1 }}$ and $C P E_{\text {Layer1 }}$ and the intact coating below the diffusion propagation front, modeled with $\mathrm{R}_{\mathrm{Layer2}}$ and $\mathrm{CPE}_{\mathrm{Layer2}}$. These elements reflect the 
different barrier properties of the two layers within the single coating and subsequent changes upon extended drying or annealing indicate developing protection of a metal as the coating coalesces. After annealing, RC-10206 showed a close fit for Model A, Figure 4.2. An increase in total impedance $|z|$ from the air dried coating to the annealed coating (approximately $10^{4}$ to $10^{7} \Omega$ at $0.1 \mathrm{~Hz}$ ), and a decrease in the capacitance of the coating (1.25 $\mu \mathrm{F}$ to $9.8 \mathrm{nF}$, calculated from and based on the fit of the $\mathrm{CPE}_{\text {coat, }} \mathrm{R}_{\text {coat, }}$ $C P E_{\text {Layer2 }}$ and $R_{\text {Layer2 }}$ elements), suggest that the barrier properties of the films against saline absorption have improved after annealing. It is commonly accepted that coatings with a total impedance of $10^{8} \Omega$ at $0.1 \mathrm{~Hz}$ demonstrate good barrier properties while those lower than $10^{6} \Omega$ generally provide inadequate protection against developing corrosion. Thus, aside from difficulties with adhesion, the air dried RC-10206 film showed less than acceptable protection against corrosion, an issue that was further explored in these studies through the use of basecoats combined with annealing. ${ }^{102,}{ }^{136}$ The relatively high capacitance of both layers of air-dried RC-10206 can be explained by incomplete coalescence of the latex particles, which may allow un-impeded diffusion through the coating due to hydrophilic pockets retained in the polymer matrix.

While (annealed) single layer films of RC-10206 had adequate protection against corrosion, the adhesion of these films to our metals of interest (e.g. bronze) is poor enough that the use of a basecoat is required. However, when films having a basecoat of B-44 with a topcoat of RC-10206 were produced, allowed to air dry for one week, and then immersed in water, the films whitened significantly, with the whitening persisting after the films were allowed to dry. That observation led us to study the whitening 
phenomenon in this two coating system more thoroughly. (No whitening was observed in single layer films of only B-44 or only RC-10206.)

Figure 4.3A shows a stereomicroscope view of a cross-section of the two-layer, water-whitened coating system and substrate. The water-whitened layer can be clearly observed in the middle of the coating. Surprisingly, given that our EIS data on air-dried $\mathrm{RC}-10206$ showed that it is more water permeable compared to $\mathrm{B}-44$, the whitened layer is not located in the RC-10206 topcoat, but rather it is in the upper part of the B-44 basecoat. The cross-sectioned films with a basecoat of B-44 and a topcoat of RC-10206 were analyzed by infrared microspectroscopy, to identify the mobile species present in RC-10206 that could be responsible for the whitening observed in the B-44 layer. The IR spectra collected from the three layers are presented in Figure 4.3B. The IR spectra in Figure $4.3 \mathrm{C}$ are reference spectra, taken from air dried single layer films, and from neat DPM. The difference spectrum, obtained from subtracting the B-44 reference spectrum from the "whitened layer" spectrum in $4.3 \mathrm{~B}$, showed that the intense peak at 1111.81 $\mathrm{cm}^{-1}$ associated with ether $\mathrm{C}-\mathrm{O}$ stretching in the DPM reference spectrum remains after subtractions.

The IR data suggested that the DPM coalescent from the topcoat formulation migrated into the acrylic basecoat because the spectrum of the whitened layer minus the spectrum from the B-44 layer yielded a spectrum similar to the reference spectrum of DPM. This data indicated that DPM played a role in film whitening since the whitened layer was exclusive to that portion of the B-44 basecoat that had absorbed DPM from the topcoat. We hypothesize that additive(s) in the RC-10206 migrated into 

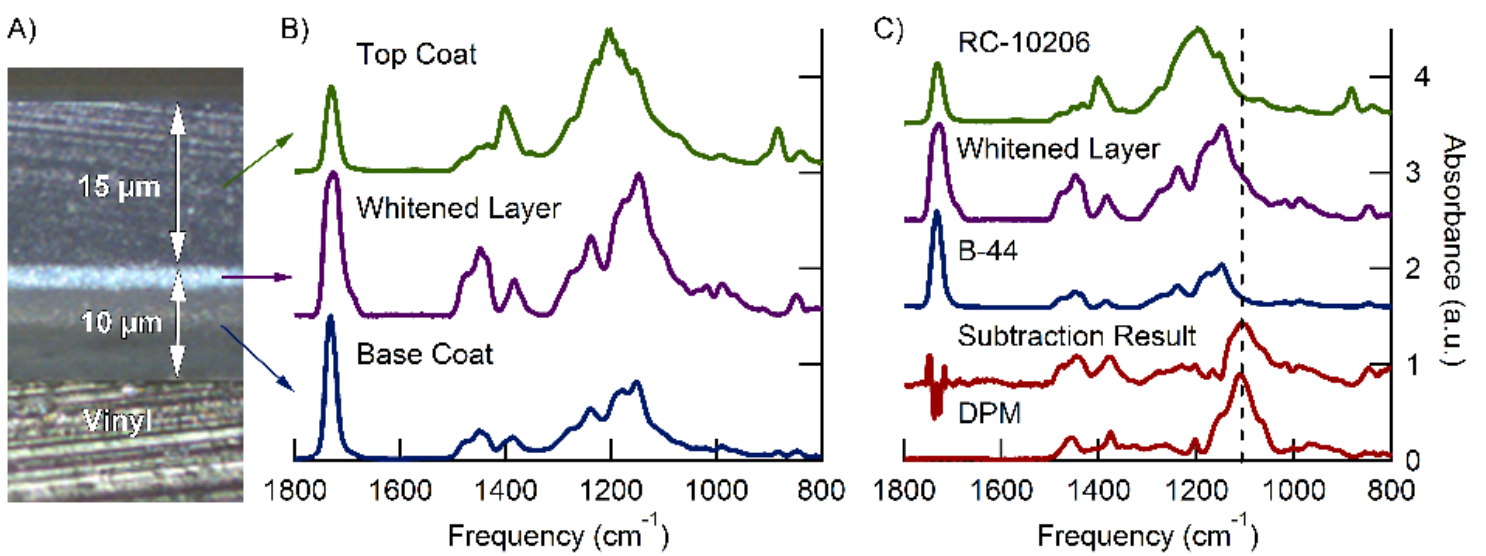

Figure 4.3 A) Stereo microscope image of a cross-section of RC-10206 (topcoat) on B-44 basecoat; after one hour immersion in water the whitened inner layer was visible; B) The micro-FT-IR spectra for each layer; C) Reference FT-IR spectra of the topcoat (RC-10206), basecoat (B-44) both white and transparent portions, DPM, and the subtraction result obtained from the "whitened layer spectrum" in $3 B$ minus the $B-44$ reference spectrum in $3 \mathrm{C}$. The ether associated C-O stretch at $1120 \mathrm{~cm}^{-1}$ from DPM is clearly present in the whitened layer after B-44 has been subtracted.

the basecoat and were retained in the basecoat layer as the topcoat formed a film.

When the films were subsequently exposed to water, water accumulated within the region of the coating which contained the coalescent. Those hydrophilic pockets having sufficient size to scatter light resulted in whitening of the films. The whitened portion of the film was inhomogeneous because only a portion of the film whitened. Since the whitened layer in Figure 4.3 did not reach the substrate, that suggested some layers did not absorb water, and the anti-corrosion properties of whitened films may be relatively unchanged.

To test this hypothesis, RC-10206 coatings were formulated with varying amounts of hydrophilic components, containing either $5 \%, 10 \%$ or $20 \%$ DPM (added as a coalescing agent). Films of these coatings were cast onto black vinyl Leneta charts and their mass uptake and grayscale values were recorded before and after immersion in water for four days, with data from this experiment shown in Figure 4.4. The dry films 


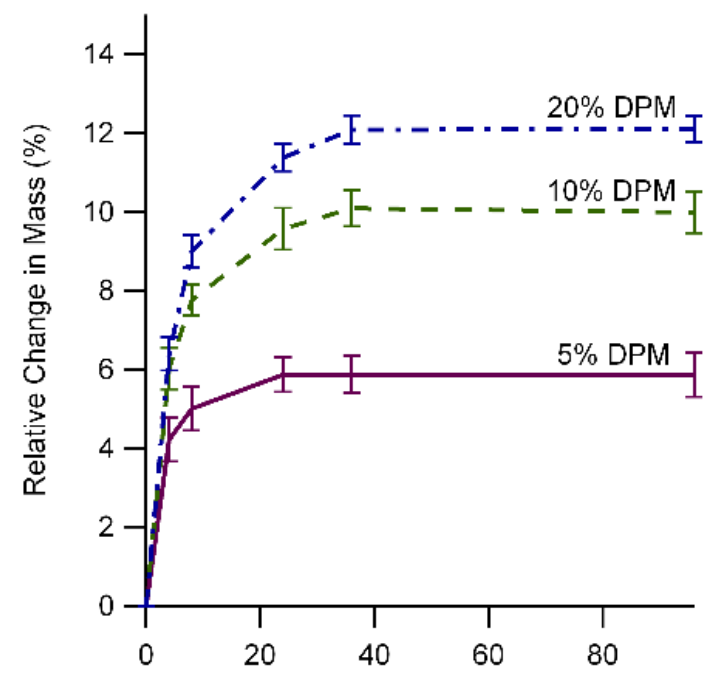

A)

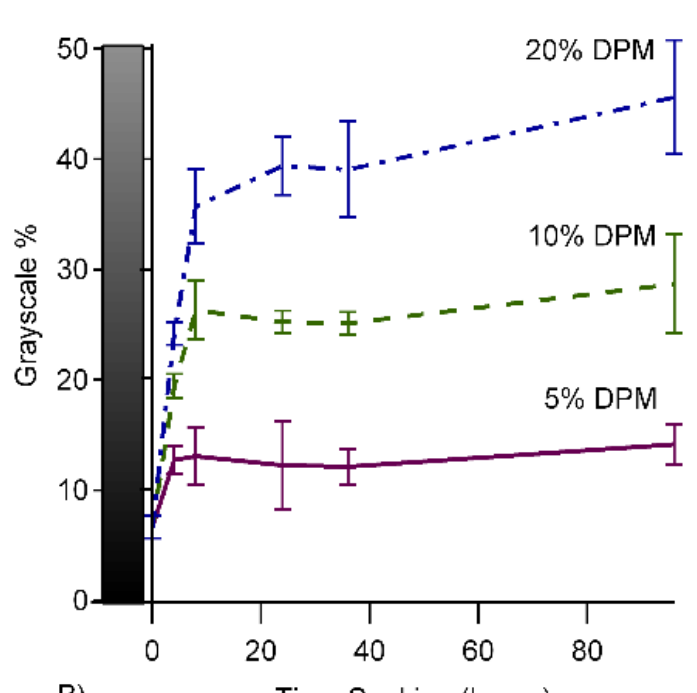

B) Time Soaking (hours)

Figure 4.4 Immersion studies of layered coatings with varying coalescent (DPM) loading levels during formulation. A) Mass-uptake over 96 hours observed for air-dried films of RC-10206 applied onto basecoats of B-44, as a function of the percentage DPM in the RC-10206 topcoat; B) Water whitening by grayscale measurements. The color of the bar indicates the level of gray from $0-50 \%$, where $100 \%=$ white.

produced by the three different formulations with varying amounts of coalescent showed similar trends in both uptake and whitening: an initial sharp increase immediately after immersion and greater \% weight of DPM in the formulation resulted in increased levels of both uptake and whitening. Before immersion in water, the films exhibited baseline grayscale values of approximately $5 \%$. Dry films that contained $10 \%$ DPM by weight in their liquid formulation showed more than twice the grayscale whitening as the $5 \%$ coalescent film, and subsequently the $20 \%$ film showed almost twice that of the $10 \%$ film (Figure 4.4B). Based on evidence in Figure 4.3, it is expected that water collected in the mid region of these studied films, but given the rapid initial whitening of the films, sorption could also occur in the near surface of the film. ${ }^{37}$ While it is evident that increased \% weight of DPM resulted in increased uptake and whitening of the films, the extent to which the protective nature of the "whole" coating 
A) with $5 \% \mathrm{DPM}$

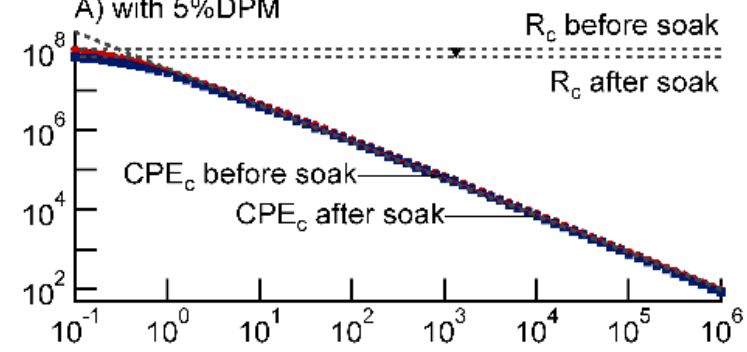

B) with $10 \% \mathrm{DPM}$

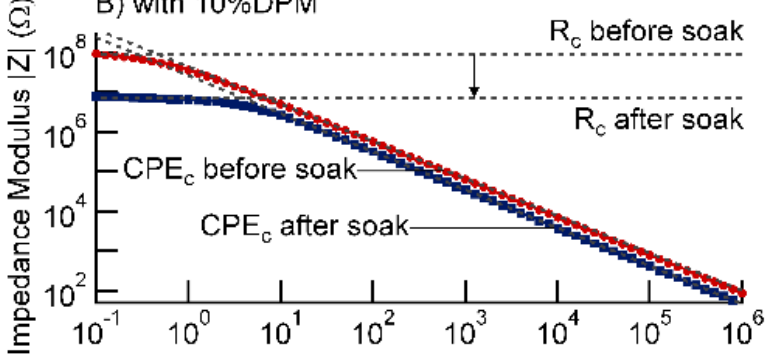

C) with $20 \% \mathrm{DPM}$

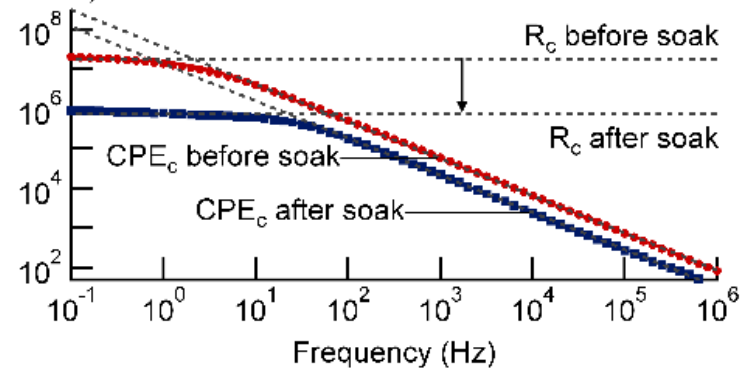

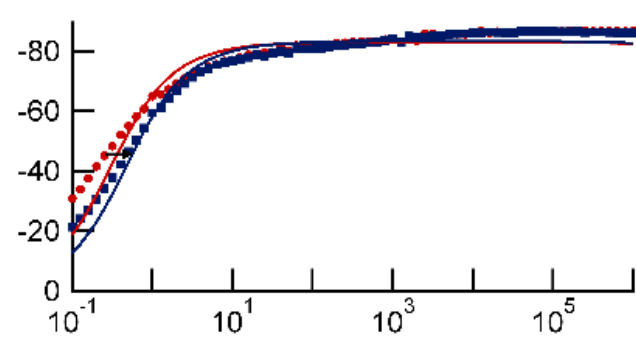
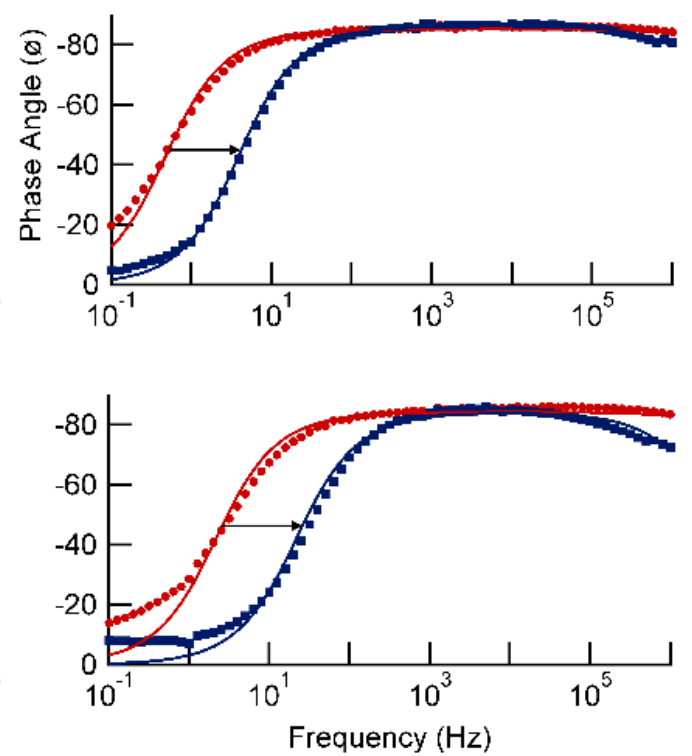

Figure 4.5 Electrochemical Impedance Spectra of RC-10206 with varying concentrations of DPM after a four hour soak. Data for B-44 base/RC-10206 topcoat dried one week was fit to equivalent circuit Model $A$ in Figure 2. A) $5 \%$ DPM RC-10206: before soaking, $R_{\text {coat }}=1.1 \times 10^{8} \pm 1.3 \times 10^{6} \Omega, C P E_{\text {coat }}=5.1 \times 10^{-9} \pm 3.6 \times 10^{-11}$ Siemens. $\sec ^{\alpha}, \alpha=0.92$; after 4 hour soak, $R_{\text {coat }}=6.8 \times 10^{7} \pm 7.2 \times 10^{3} \Omega, C P E_{\text {coat }}=5.4 \times 10^{-9} \pm 4 \times 10^{-11}$ Siemens. sec $^{\alpha}$, $\alpha=0.93$ B) $10 \%$ DPM RC-10206: before soaking, $R_{\text {coat }}=8.7 \times 107 \pm 9.3 \times 10^{5} \Omega, C P E_{\text {coat }}=4.1 \times 10^{-9} \pm 4.0 \times 10^{-11}$ Siemens. $\sec ^{\alpha}, \alpha=0.95$; after four hour soak $R_{\text {coat }}=7.0 \times 10^{6} \pm 5 \times 10^{4} \Omega, \quad C P E_{\text {coat }}=6.6 \times 10^{-9} \pm 6.5 \times 10^{-11}$ Siemens. $\sec \alpha, \alpha=0.96$ C) $20 \%$ DPM RC-10206: before soaking, $R_{\text {coat }}=1.7 \times 10^{7} \pm 1.3 \times 10^{5} \Omega, C P E_{\text {coat }}=5 \times 10^{-}$ ${ }^{9} \pm 4.5 \times 10^{-11}$ Siemens. $\sec ^{\alpha}, \alpha=0.94 ;$ after soaking $R_{\text {coat }}=7.6 \times 105 \pm 4.5 \times 10^{3} \Omega, C P E_{\text {coat }}=1.3 \times 10^{-8} \pm 1.7 \times 10^{-10}$ Siemens $\cdot \sec ^{\alpha}, \alpha=0.935$.

and possible changes in film formation/morphology were affected by these phenomenon needed to be determined, which was subsequently done using EIS.

EIS was performed on the same coating layers that produced significant whitening, but applied on bronze substrates: B-44/RC-10206 films containg 5\%, 10\%, and $20 \%$ DPM as a coalescent and soaked for four hours. The EIS data (markers), the equivalent circuit models used in data fitting (dashed lines), and the best fit lines (solid 
lines) are shown in Figure 4.5 with calculated time constants and volume fractions of water in Table 4.1. The RC time constant, $\tau_{R C}$, was calculated as the product of the resistance and capacitance of the coating using Model A in Figure 4.2. The RC time constant occurs at a phase angle of $-45^{\circ}$, and is the half-way point between capacitive behavior $\left(-90^{\circ}\right)$ and resistive behavior $\left(0^{\circ}\right)$. Its position on the $x$-axis is useful for comparing the frequency of the transition from resistive to capacitive behavior of different films and the speed in which ions travel from the bulk solution to the metal substrate.

Before soaking, films produced from colloidal resin suspensions containing $20 \%$ DPM exhibited the fastest time constant (0.0871 seconds) compared to those produced from 10\% DPM (0.357 seconds) and 5\% DPM suspensions (0.547 seconds). The permeability of films to water absorption corresponded to changes in its RC time constant, with faster time constants that were produced by both a decrease in resistance and increase in capacitance of the coating. The trend observed in Table 4.1, that increased weight percent of DPM in the wet coating resulted in a decreased RC time constant of the dry film, suggested that "dry" films made from formulations containing higher levels of DPM are more water-permeable than those made with lower DPM levels.

\begin{tabular}{llll}
\multicolumn{4}{l}{ Table 4.1 Water uptake in topcoats with varying amolunte of DPM } \\
\hline \%DPM & $\begin{array}{l}\tau_{\mathrm{RC}, \mathrm{t}=0}(\mathrm{~s}) \\
\text { before soak }\end{array}$ & $\begin{array}{l}\tau_{\mathrm{RC}, \mathrm{t}=4 \mathrm{~h}}(\mathrm{~s}) \\
\text { after soak }\end{array}$ & $\begin{array}{l}\mathrm{VF} \mathrm{H} \mathrm{H}_{2} \mathrm{O}(\% \mathrm{~V} / \mathrm{V}) \\
\text { After soak }\end{array}$ \\
\hline 5 & 0.547 & 0.364 & $0.677 \pm 0.0845 \%$ \\
10 & 0.357 & 0.0464 & $9.22 \pm 0.412 \%$ \\
20 & 0.0871 & 0.101 & $18.1 \pm 1.87 \%$ \\
\hline \multicolumn{4}{l}{ Water uptake was measured } \\
drawn into the layered coating during EIS after a four hour soak.
\end{tabular}


The DPM concentration was varied (data shown in Figure 4.5) and data from each concentration fit using EEC Model A (model shown in Figure 4.2). Using the modeled fit of the $\mathrm{CPE}$, a theoretical value of $\mathrm{C}_{\text {coat }}$ was calculated. Based on the data fits in Figure 4.5 and calculated values of capacitance, the volume fraction of water in the films after soaking can be estimated using Equation 4.1. The volume fractions (VF) calculated were $0.677 \pm 0.0845 \%$ for $5 \%$ DPM topcoats, $9.22 \pm 0.412 \%$ water in $10 \%$ DPM topcoats and $18.1 \pm 1.87 \%$ when $20 \%$ DPM was used in the coating formulation. The results from this calculation follow the same trend and interpretation: that increasing percentages of DPM in the formulation in the topcoat produce films that are more permeable to water and contain larger volume fractions of water. Considering the case of the film produced from $20 \%$ DPM, while the calculated water volume fraction (18.1\%) may seem unrealistically high these films have been observed to blister and swell, suggesting that such copious amounts of water were indeed occupying the films. In contrast, the volume fraction calculated for the $5 \%$ DPM film by EIS was less than $1 \%$ as compared to the almost $6 \%$ mass change in Figure 4.4. This increase in mass may be attributed to water trapped between the vinyl substrate - coating interface. EIS allowed soaking of the film over a limited area, whereas immersion studies allowed water transport through the sides and surface of the film, which may have permitted the coating to saturate more quickly and increase the uptake at similar time points. Despite slight variations in quantifying the amount of water absorbed by the films, the data trends by EIS,VF calculations, mass increase and grayscale changes are consistent: that increased whitening of the films corresponds to increased water sorption in the films 
which is related to higher loading levels of coalescent in the formulation. Interestingly, while the data is not shown, less hydrophilic coalescents, such as Texanol, showed no greater resistance to mass uptake or water whitening, suggesting that in the process of latex film formation, excess coalescent is forcibly excluded from the topcoat into the basecoat. This generalized observation may support our earlier work on the role of film formation from different types of coatings: those dissolved in solvent versus aqueous colloidal suspensions. ${ }^{37}$

\subsubsection{Effect of basecoats and annealing}

Although B-44 was specifically selected as the basecoat for our studies because of its widespread use as a good protective coating for metals, we tested different solventbased basecoats with RC-10206 topcoats to compare their water whitening susceptibility. Films composed of the resin A-21 $\left(\mathrm{Tg}=105^{\circ} \mathrm{C}\right)$ were compared with films containing $75 \%$ B- 44 and $25 \%$ A-21 (estimated $\mathrm{Tg}=71^{\circ} \mathrm{C}$ ), and B- 44 only $\left(\mathrm{Tg}=60^{\circ} \mathrm{C}\right.$ ). The pure B-44 film whitened the most and was $60 \%$ gray after immersion in DI water (shown in Figure 4.6A). In contrast, the pure A-21 film did not exhibit whitening above baseline gray level (showing a $7 \%$ gray value after immersion). Intermediate levels of whitening were observed with mixtures of these two resins as the basecoat. For example, 3:1 B-44:A-21 mixture showed approximately $20 \%$ gray after immersion in water. Most films returned to baseline gray levels after ten hours of drying, but the films with only a B-44 resin base coat required a minimum of 30 hours. While these results indicated that the whitening effect was reversible, it also required many hours of 
dry conditions to do so. Films that lacked a topcoat of RC-10206 did not whiten at all: films of entirely B-44 or A-21 or mixtures of these did not whiten above baseline gray, and neither did single-layer films of RC-10206; whitening was only observed when topcoats of RC-10206 were used in combination with some solvent-based basecoats. This observation supports the hypothesis that a mobile component (the coalescent) in the RC-10206 caused water whitening when it was observed. These experiments showed that the basecoat's glass transition temperature has an effect on the extent of whitening, but is not the cause of the observed whitening. (The films' chemistry is similar as they are polymers of MMA or EA).
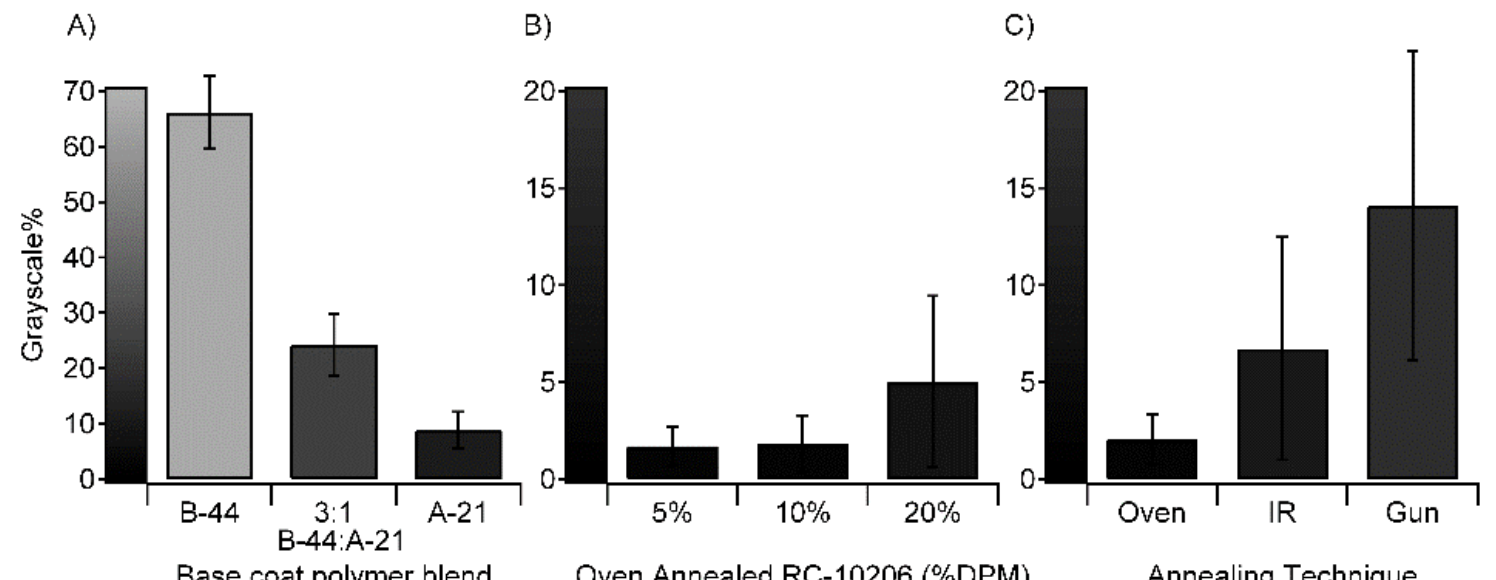

Base coat polymer blend

Oven Annealed RC-10206 (\%DPM)

Annealing Technique

Figure 4.6 Grayscale values found during immersion studies of vinyl with RC-10206 topcoat and various basecoats after immersion in room temperature water for four hours. The color of the bar indicates the level of gray from $0-100 \%$, where $100 \%=$ white. A) Whitening as a function of different solvent basecoats in A-21 / B-44 study B) Whitening of annealed B-44/RC-10206 films coalesced with various DPM levels C) Comparison of whitening after various annealing techniques with 10\% DPM RC-10206 topcoat and B-44 basecoat.

Though our results show that water whitening can be reduced by changing the composition of the basecoat, that strategy may be impractical for outdoor coatings applications because of B-44's desirable properties as an outdoor coating: moderate $\mathrm{Tg}$ for good flexibility under a wide range of temperatures and strong adhesion to metals, 
such as cupreous ones. Therefore, experiments that could prevent, or reverse, the whitening effect observed using a B-44 basecoat were conducted. Heating of DPMcontaining films was expected to speed the evaporation of DPM (vapor pressure of 37.3Pa at $20^{\circ} \mathrm{C}$ ). Shown in Figure $4.6 \mathrm{~B}$ are $\mathrm{RC}-10206$ films with $\mathrm{B}-44$ as a basecoat and, for a comparison, three different loading levels of DPM after they were oven annealed. Regardless of the amount of DPM, annealing for one hour resulted in grayscale levels similar to the 5\% DPM air dried sample. While oven annealing was extremely effective at reducing water whitening, that method is impractical for use on large or fixed-inplace metal objects that cannot be placed into an oven. Practical methods for onsite applications to drive off excess coalescent after films had been applied, such as heating by heat gun or an infrared lamp, were investigated with results shown in Figure 4.6C. All methods reduced whitening compared to films that were allowed to air dry without annealing: the heat gun was the least affective annealing method ( $14 \%$ gray), while the IR lamp and oven did not whiten above baseline levels (less than $8 \%$ gray). These data show that two methods of annealing, by oven $\left(60^{\circ} \mathrm{C}\right.$ for one hour) and IR lamp $\left(60^{\circ} \mathrm{C}\right.$, four hours), are equivalent and equally effective at preventing water whitening. Annealing by heat gun was less effective than oven or IR lamp, but did reduce the amount of water whitening of films treated by that method. For most applications, such as coatings for buildings and sculptures, it is impractical to suggest post-coating annealing by oven as a method to prevent water-whitening, however annealing by IR lamp is a practical alternative and will be explored further in future work. 


\subsubsection{Water sorption characterized by EIS}

Films with basecoats of $\mathrm{B}-44$ and topcoats of $\mathrm{RC}-10206$ were produced on bronze panels with gold foil electrodes placed in between the layers (as shown in Figure 4.1B). The use of interlayer electrodes allowed EIS characterization in Figure 4.7 of the individual layers in a two-layer coating system. The total impedance of RC-10206 topcoats only was lower at frequencies less than $10^{4} \mathrm{~Hz}$ compared to the total impedance of both layers together. That general observation applies to films that had been annealed or were allowed to air dry, however the differences were much reduced after heat treatement. After annealing, the total impedance modulus through both coatings compared to that through the topcoat alone showed that the barrier properties of the topcoat were nearly as high as through both layers, where the phase angle plots showed near ideal capacitance at $-90^{\circ}$. Another indication of improved barrier properties with drying can be seen with the shift in the first RC time constant of the 4 weeks air dried film from approximately $10 \mathrm{~Hz}$ to $0.01 \mathrm{~Hz}$ (by extrapolation) with an annealing treatment. It is likely that annealing drives off remaining additives and completes film formation, forcing water/electrolyte to take a slow (i.e. longer RC time constant) path from the bulk solution to the metal. Thus annealing both improves coatings' barrier properties (i.e. its long-term performance) and makes them less permeable to water/electrolyte (i.e. its short-term performace). 


\subsection{Discussion}

When DPM was used in high levels as a coalescing aid for the Kynar ARC RC-10206 resin, depending on the hardness of the basecoat, DPM may migrate into that layer producing a softened hydrophilic region. From our EIS data we cannot deduce how the DPM is distributed in the films: whether it is dispersed evenly throughout, or whether it is aggregated into regions that could produce large hydrophilic pockets.
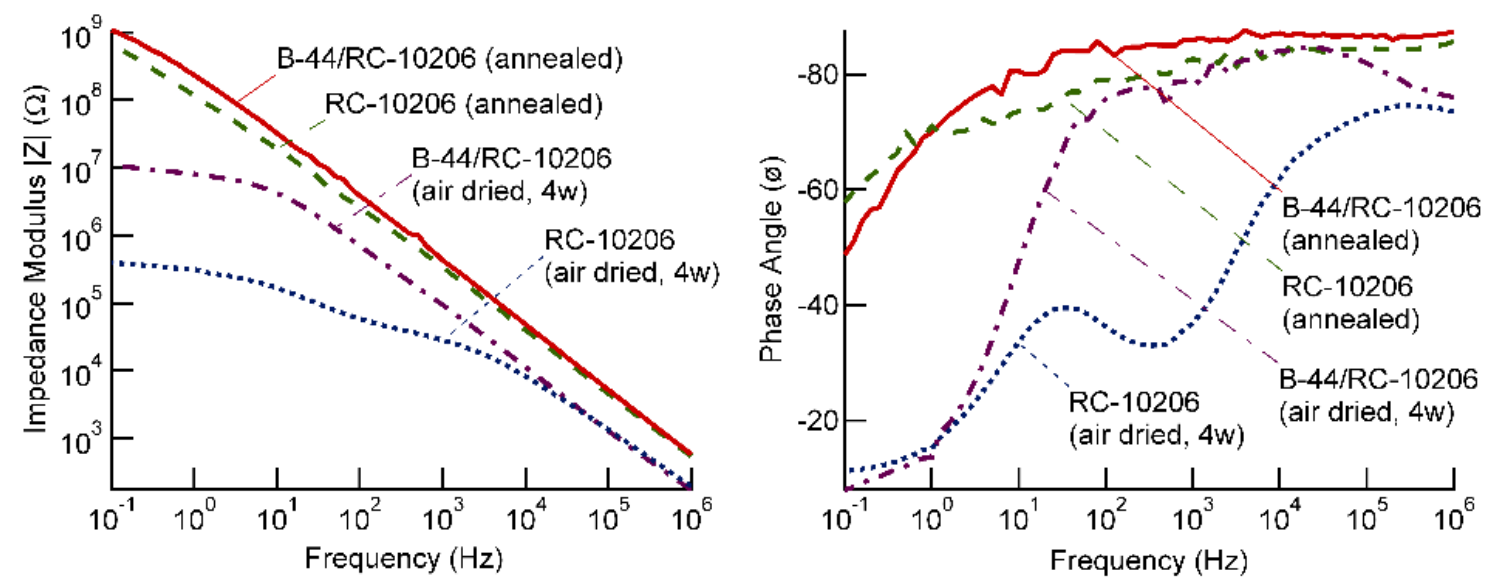

Figure 4.7 Improved barrier properties of coatings with longer drying times and annealing. Basecoat of B-44 and topcoat of RC-10206 layered systems air-dried four weeks or annealed in an oven for $16 \mathrm{~h} 60^{\circ} \mathrm{C}$. Interlayer electrodes were used to obtain EIS spectra of RC-10206 alone.

The cross-sectional view of a layered acrylic coating of B-44 and RC-10206 in Figure 4.3A showed the white layer present only in the upper portion of the basecoat, suggesting that some of the residual DPM is sequestered within the upper layer of the basecoat. The coating resistance, $R_{\text {coat, }}$ at $0.1 \mathrm{~Hz}$ of the two formulations containing either $10 \%$ or $20 \%$ DPM, decreased significantly upon water immersion bringing the total impedance modulus below the acceptable threshold of $10^{6} \Omega$ at $0.1 \mathrm{~Hz}$ without having annealed the films (see Figure $4.5 \mathrm{~B}$ and $4.5 \mathrm{C}$ ). Also of note was the initial resistance of the coatings, which decreased when DPM concentration was increased. 
Retained coalescing agents may have created hydrophilic pockets that allowed electrolyte to travel freely and increase capacitance, but may have also impeded latex coalescence, preventing a solid polymer network from forming. Waterborne coatings do not create the same tight-packed polymer network that solvent-based polymer formulations do, leaving more opportunities for pores and other defects ${ }^{37}$. The amount of coalescent present in the coating formulation affected the permeability of the films as characterized by electrochemical, mass uptake and visual changes.

Calculations from Equation 4.1 are based on completely random yet evenly dispersed inclusion of water in a polymer network of sphere like particles in the film. A dense, close-packed system of spheres would provide nearly $66 \%$ of the solid volume fraction in the film. Because resins are conformable spheres, it is likely that the interstitial space is somewhat less than $33 \%$, however a water volume fraction of almost 18\% (as measured by EIS) seems large for films that have not been water saturated. In comparison, our direct measurements of water uptake showed an increase in mass of $6.4 \%$ over the same time period ( 4 hours). Interestingly, Brasher and Kingsbury concluded that distribution of water molecules in column-like pores perpendicular to the surface (as opposed to random dispersion) would, in fact, result in a VF estimation that is larger than what a random distribution of water molecules would produce. ${ }^{124}$ Pore channels already exist due to the imperfect film formation of waterborne coatings (which in this case may be aggravated by the quantity of coalescent in the formulation) and combined with theoretical models of water uptake, a compelling argument 
develops that water does not diffuse into a coating in a random, uniformly-distributed fashion.

Because all of our best fitting EEC include CPEs instead of ideal capacitors, the presence of CPEs provides additional support for the existence of a multitude of similar but not equivalent pathways for water/electrolyte ingress due to the non-homogeneity of the coating. As was observed in the impedance data shown in Figure 4.2, water reached only part way through the film creating a swollen top layer and leaving a nonswollen layer of coating near the metal.

It is likely that the small blisters that formed upon long immersions held large amounts of water (which in part, accounts for the large estimated VF of water). Although damage to the metal was not observed during any of the experiments in this study, we acknowledge that the long-term performance of the coating system may be decreased by the softening of a portion of the base coat and this idea will be explored in future work. Our results show that basecoat choice is an important factor for protecting metal substrates against water/electrolyte penetration leading to corrosion.

Basecoats of B-44 resin may be used in combination with a topcoat of RC-10206 for adequate protection when the films are annealed, with each layer exhibiting excellent barrier properties, as seen in Figure 4.7. The low impedance of un-annealed films containing RC-10206 as a single layer or as a topcoat is likely explained by incomplete film formation. Films that have not fully formed into a homogeneous polymer network likely have micro- or nano-scopic defects as pores or polymer voids 
that may swell with water; structural characterization of the pores is a topic for further study.

The susceptibility of films to water-whiten is one of many indicators of coating failure but it is as yet unknown how this problem affects the long-term barrier properties of the coating. It is likely that solvents and coalescing agents responsible for the whitening phenomenon are driven off naturally if sufficient time is allowed or if hydrophilic components are leached out of the film by repeatedly saturating it with water. Unfortunately, while only a few hours are needed for bulk water evaporation and coalescence, depending on drying conditions, more than 30 days may be necessary for complete coalescence (i.e. latex particle fusion) and the complete evaporation of the residual coalescing agent $(<1 \%) .{ }^{37,}{ }^{137-138}$ This extended drying time line may be impractical for most outdoor applications as corrosion to the substrate can occur during that time.

A fine-tuning of the PVDF hybrid waterborne dispersion would therefore seem to be a more productive route to achieving excellent protective coatings for bronze. In particular, the use of adhesion promoters to achieve direct adhesion to bronze, would potentially allow for single coat waterborne applications with much lower overall system VOC levels. Likewise, reducing the minimum film formation temperature of the PVDF dispersion to below ambient temperature would permit late stage film formation to occur in the field, without a separate annealing step and without the need for large quantities of coalescent. 


\subsection{Conclusions}

Analysis of circuit elements and defects of various coatings and the application to EIS remains our most promising avenue to both understand and improve commercial coating formulations for use in certain applications for which there exist no products specifically produced for their protection, such as coatings for outdoor bronze sculptures. It was postulated, and our data seems to confirm, that the whitening phenomenon was caused by a migration of mobile DPM from the topcoat into the basecoat, softening it and allowing water accumulation in that layer. Both visual observations and electrochemical analysis showed improved performance when a solvent-based primer was used with the waterborne topcoat. The permeability of films of RC-10206 topcoats with B-44 basecoats may be reduced by annealing treatments, with use of an IR lamp as a practical alternative for oven annealing. Films annealed by those means showed extremely high impedance, exceeding $1 \mathrm{G} \Omega$ at $0.1 \mathrm{~Hz}$ and as such can be considered to offer excellent protection against corrosion. 


\section{Investigating the protective lifetime of novel waterborne and traditional solvent-based coatings for the protection of outdoor metalworks}

Given the high cultural value of outdoor sculpture there is a great need to fully test new types of polymeric coatings under consideration for the protection of such works using relevant alloys of bronze and steel. Fluoropolymer based coatings are well-suited for outdoor use due to their resistance to damage by UV light and as such may be suitable for use on sculptures. Waterborne polymer resins comprised of primarily polyvinylidene fluoride and polyacrylics were formulated as clear-coatings, applied on bronze and steel test panels and subjected to outdoor weathering in a standard test site in south Florida, in Portland, $O R$ and in a QUV-B chamber. The performance of those relatively novel fluoropolymers was compared to traditional acrylic coatings such as solvent-based Paraloid B-44 and Incralac, and a waterborne coating for outdoor use, WL-81. The effects of common additives to prevent corrosion and confer resistance to degradation by UV-light were investigated. Performance characteristics of all coatings were monitored before and throughout weathering by Electrochemical Impedance Spectroscopy and Fouriertransform infrared microspectroscopy. Chemical and mechanical changes in the polymer films identified through spectroscopic means are expected to provide insight for the possible use of such fluoropolymer coatings on outdoor sculpture. Coatings were evaluated for workability and gloss in a blind study involving U.S. conservators, which showed that the novel PVDF coating had acceptable qualities. Evidence presented here suggests that a waterborne PVDF-acrylic top coat (Kynar Aquatec ARC or FMA) on a thin PMMA/PEA 
primer coat dramatically outlasts the performance of the control PMMA/PEA coating (Paraloid B44) and is both a viable and sustainable alternative to the current wax and solvent-based coatings systems (e.g. Incralac) preferred in cultural heritage conservation.

\subsection{Introduction}

The aesthetic viability and barrier properties of a coating are of equally high import for the conservation of culturally significant outdoor metal works. Current coatings used in the conservation of outdoor metalwork have a lifetime expectancy of one year for waxes and two to three years for solvent-based coatings before stripping and reapplication. ${ }^{20}$ By extending the lifetime of a coating to ten years or more and prolonging time between applications, costs of materials and labor would decline, and would concomitantly aid in more sustainable coating use practices. As society moves towards a more sustainable existence, both legislation and ethics will urge the conservation community to make more environmentally-friendly choices for protective materials of cultural heritage. ${ }^{14}$ To accomplish those goals coatings must reduce the volatile organic component ( $\mathrm{VOC}$ ) of the resin as well as resist damage from the environment they are placed in, i.e. outdoor weathering, by creating materials with hydrophobic properties as well as high thermal and photochemical stability. Ethical requirements in conservation treatments also require transparent, low-gloss and reversible coatings, limiting applicable materials to waxes and thermoplastic resins. For this application, a coating must meet the following criteria, abbreviated as TRACE: optically Transparent, Reversible, easily Applied, Commercially available, and 
Environmentally sustainable. ${ }^{54}$ This work investigates the suitability of waterborne acrylic and fluorinated latex polymer resins to meet the TRACE criteria and surpass the protective lifetime in comparison with traditional solvent-based materials such as Incralac ${ }^{\circledR}$ and Paraloid ${ }^{\circledR}$ B-44.

The protective nature of an organic coating is most simply related to its ability to inhibit the transport of ions/vapors/gas through the film to the substrate. Polymers make good coatings not only due to their ability to be plasticized and reform over a variety of topographies, but also because water vapor has low solubility in non-polar materials. Waxes are among the most widely used coatings in conservation because they are relatively hydrophobic, have low gloss, and are somewhat reversible, fairly inexpensive and easy to apply. However, we have previously shown waxes alone are readily susceptible to oxidation and offer insufficient protection during outdoor weathering for a number of chemical and microstructural reasons [See Chapter o]. Since waxes do not weather evenly, some degradation products in waxes could also promote the early-onset of degradation of other polymers, and use as an aesthetic top coat may actually promote the onset of localized degradation and eventual damage of the underlying substrate. Conservators sometimes apply waxes on top of solvent-based acrylic coatings, such as Incralac (principle component of which is Paraloid B44), to provide a more matte finish. Others have reported that those systems have reasonable barrier properties by electrochemical analysis methods. ${ }^{9,20,139}$

Numerous studies have focused on the chemical analysis of acrylic polymer degradation. ${ }^{13,}{ }^{140-147}$ In recent years research has focused on identifying analytical 
signatures to track chemical changes that may serve as precursors to gross film failure. Physical degradation of coatings can be seen as changes in tensile strength, shape, color, or clarity. The ability of coatings to prevent corrosion can depend upon the chemistry and durability of the polymer, especially its ability to resist degradation due to UV light exposure. ${ }^{42,47-48}$ Organic coatings are susceptible to changes in chemical species by chain scission, cross-linking, recombination and oxidation of polymer side groups. A buildup of these products can be characterized using methods sensitive to organic bond changes, such as infrared spectroscopy, and are expected to precede macroscopic polymer degradation (or bulk failure). Acrylic and methacrylic polymers with short alkyl side chains can undergo chain scission, cross-linking, and loss of monomers, but little infrared evidence has been found for these events during natural aging. ${ }^{143-145,}{ }^{147-148}$ How and why certain acrylics degrade remains an open research question, but a general consensus from the literature is that "unzipping" or chainscission can occur, catalyzed by impurities and or residual activator in the resin. ${ }^{149-156}$

Fluorinated polymer hybrid blends based on poly(vinylidene fluoride) (PVDF) are among the most UV-resistant resin systems used today in commercial coatings for metals, with protective properties lasting in many cases for more than thirty years of outdoor exposure, as described by Wood et al. and others. ${ }^{13,}$ 48-49 Traditional PVDFcontaining coatings were often formulated at high VOC levels but recent advances have produced waterborne (latex) hybrid dispersions with high PVDF resin content, and the performance of these coatings continues to improve.$^{50,52}$ Commercial products of this type contain $70 \%$ or $50 \%$ PVDF on total resin weight, with the remainder of the resin 
being polyacrylics, the ratios of which are similar to their solvent-based counterparts. ${ }^{11}$ ${ }^{47}$ Because of their relatively inert chemistry, PVDF coatings do not provide good adhesion to metals compared to acrylics such as a solvent-based methyl methacrylate/ethyl acrylate copolymer (PMMA-co-PEA). ${ }^{53}$ In our previous work, we circumvented the adhesion problem (and reversibility concerns of colloidal polymers) by using an acrylic basecoat (for good adhesion and reversibility) combined with a waterborne PVDF-based topcoat (for good weatherability) and observed excellent intra-layer adhesion. Like solvent PVDF coatings, waterborne PVDF coatings of this type have been shown to offer superior barrier properties, after exposure to UV light, compared to solvent-based $100 \%$ acrylic coatings such as poly(methyl methacrylate) and ethyl acrylate. ${ }^{11,} 157$ These three polymers along with poly(n-butyl acrylate) and polystyrene are the basis of this work, the chemical structure of each is depicted in Scheme 5.1, with trade names and physical properties listed in Table 5.1.

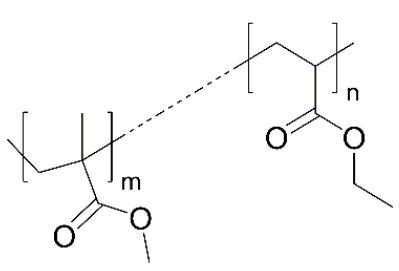

poly(methyl methacrylate-co-ethylacrylate)

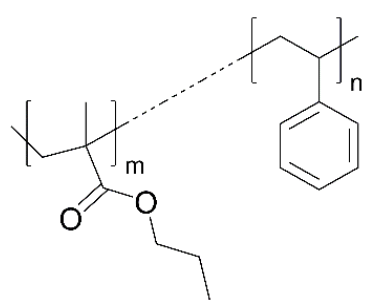

poly(n-butyl methacrylate-co-styrene) polyvinylidene fluoride

Scheme 5.1 Representations of poly(methyl methacrylate-co-ethyl acrylate) (PMMA-co-PEA) and poly(vinylidene fluoride) (PVDF) where $m, n$, and $q$ indicate the number of repeating units

Electrochemical impedance spectroscopy (EIS) is a non-destructive method for evaluating long-term performance and as such is ideal for comparing degradation of protective coatings exposed to the same natural and accelerated weathering 
conditions. We have previously used EIS to characterize differences in film formation (polymer network integrity) of solvent-based and waterborne coatings, to monitor the barrier properties of weathered waxes on bronze and steel, and have developed a method to monitor the protective quality of coatings on sculptures.

Table 5.1 Properties of polymers utilized in this study

\begin{tabular}{|c|c|c|c|c|}
\hline Polymer & Trade Name & $\begin{array}{l}\text { \% solids } \\
\text { (in solvent) }\end{array}$ & $\begin{array}{l}\text { TGA/MFFT } \\
\left({ }^{\circ} \mathrm{C}\right)\end{array}$ & $\begin{array}{l}\text { Diffusion } \mathrm{H}_{2} \mathrm{O} \\
\text { in film }\left(\mathrm{cm}_{2} / \mathrm{s}\right)\end{array}$ \\
\hline Wax & ance Wax & $20 \%$ (naphtha) & $\mathrm{n} / \mathrm{a}$ & $5.01 \times 10-9$ \\
\hline PMMA-CO-PEA & Paraloid B-44 & $30 \%$ (in tolvene) & 60.1 & $1.46 \times 10-10$ \\
\hline PnBA-co-PS & Rhoplex WL-81 & $42 \%$ (in water) & 56.3 & $2.25 \times 10-10$ \\
\hline 50\%PMMA:50\%PVDF & Kynar Aquatec FMA & $46 \%$ (in water) & 12.0 & $4.78 \times 10-10$ \\
\hline 30\%PMMA:70\%PVDF & Kynar Aquatec ARC & $46 \%$ (in water) & 27.0 & $3.95 \times 10-10$ \\
\hline
\end{tabular}

In this work, bronze or steel coupons were spray-coated with 35 organic coating combinations to produce a total of 235 panels that were then subjected to various types of weathering and the film's protective quality was monitored. The matrix of panels included traditional protective coatings such as Renaissance Wax, Paraloid B44 and Incralac and more environmentally-friendly waterborne alternatives such as Rhoplex WL-81 and a coating under development by its manufacturer, Kynar ARC. Coatings on both substrates were split into three groups to undergo weathering under standard accelerated UV conditions, South Florida for standard intense outdoor testing or under milder outdoor testing in Portland, OR, the results of which have been largely disseminated here and in previous works. ${ }^{37,} 89$ In the present study, structural/chemical changes in the solvent- and waterborne polymer coatings are evaluated by FT-IR microscopy ( $\mu \mathrm{FT}$-IR) and compared to physical changes in film integrity discerned from electrochemical data. From this work, we aim to establish whether or not waterborne 
coatings containing PVDF can outlast and/or outperform waterborne and solventbased acrylic-only for the protection of outdoor metalwork.

\subsection{Experimental}

\subsubsection{Materials and sample preparation}

Basecoat primers were either Paraloid ${ }^{\circledR}$ B-44 resin dissolved in solvent [B 44, principally composed of poly(methyl methacrylate-co-ethyl acrylate), in $20 \%$ (w\%/w toluene), Dow, Inc.] or waterborne Rhoplex ${ }^{\circledR}$ WL-81 latex [WL81, poly(n-butyl methacrylate) and polystyrene, Dow, Inc.]. If a topcoat was used, it was waterborne Kynar Aquatec ${ }^{\circledR}$ RC10206 latex [ARC, poly(vinylidene fluoride) and poly(methyl methacrylate) blend, Arkema, Inc.]. Bronze substrates $(2.54 \times 7.62 \mathrm{~cm} ; 90 \% \mathrm{Cu} 10 \% \mathrm{Sn}$, TB Hagstoz \& Son Inc.) were pre-treated to prevent flash rust with a $24 \mathrm{~h}$ immersion of $17 \mathrm{mM}$ benzotriazole (Alfa-Aesar) in ethanol. BTA was also dissolved in tolvene or ethanol to add to some B-44 or WL-81 resins, respectively, for a final loading weight of $1 \%(\% \mathrm{w} / \mathrm{w})$. Steel substrates with a direct application of WL-81 were pre-treated to prevent flash rust by immersion of $20 \mathrm{mM}$ sodium decanoate (Sigma-Aldrich) in ethanol. Substrates were spray-coated with a Fuji HVLP Super $4 \mathrm{XPC}^{\mathrm{TM}}$ for a dry film thickness of approximately 10-15 $\mu \mathrm{m}$ for each layer (i.e. primer base coats or WB PVDF topcoat) for a total dry film thickness of approximately zo $\mu \mathrm{m}$. All samples were annealed (heated) in an oven for six hours at $60^{\circ} \mathrm{C}$. 


\subsubsection{Accelerated weathering exposure}

Substrates were exposed for $250 \mathrm{~h}$ time periods in a Q-Lab QUV chamber and weathered according to ASTM G154 Cycle 2: a 50oh cycle consisting of 4 hours of UV-B exposure at $60^{\circ} \mathrm{C}$, irradiance $=0.78$ and 4 hours of condensation at $50^{\circ} \mathrm{C}$ with distilled water. Images were acquired with a stereoscopic light microscope (Leica MZ6). Gloss and thickness measurements were acquired as an average of five trials with a Gardco $\mu$ Tri-Gloss meter. Differential scanning calorimetric thermograms were acquired with a Mettler TA 3000 at a rate of $30 \mathrm{~K} / \mathrm{min}$ under Argon flow. Differential Scanning Calorimetry scans were acquired with $10-15 \mathrm{mg}$ of the coating in aluminum pans with a Mettler TA 3000 DSC at a rate of $30 \mathrm{~K} / \mathrm{min}$.

\subsection{Electrochemical impedance spectroscopy}

Measurements were collected with Gamry Reference 6oo Potentiostat and Gamry Framework software. EIS measurements were performed with a standard threeelectrode Gamry Paint cell set up: a glass cell filled with $3 \% \mathrm{NaCl}$ was clamped to a coated bronze panel acting as the working electrode, the $\mathrm{Ag} / \mathrm{AgCl}$ reference electrode and graphite counter electrode were immersed in the electrode solution with a $4 \mathrm{~cm}$ distance between the counter and working electrodes. An area of exposure of the working electrode equal to $14.6 \mathrm{~cm}^{2}$ was used for analysis from $1 \mathrm{MHz}$ to $0.1 \mathrm{~Hz}$ with $\mathrm{AC}$ voltage $20 \mathrm{mV}$ rms, and DC voltage $0.0 \mathrm{~V}$ vs. open circuit potential $-200 \pm 80 \mathrm{mV}$. Spectra were interpreted in IGOR. Average potentiostat instrument error ranged from $10 \%$ at $0.1 \mathrm{~Hz}$ to less than $0.5 \%$ at $1 \mathrm{MHz}$. 


\subsubsection{Fourier-transform $\mu$-infrared spectroscopy}

A ThermoScientific iS10 infrared spectrometer with a Nicolet Continu $\mu m$ FT-IR microscope and 250 $\mathrm{mm} \mathrm{MCT} \mathrm{(mercury} \mathrm{telluride/cadmium} \mathrm{telluride)} \mathrm{detector} \mathrm{was} \mathrm{used}$ to acquire each spectrum from $4000-650 \mathrm{~cm}^{-1}$ with $4 \mathrm{~cm}^{-1}$ resolution using Omnic software. Data was transformed using an N-B strong apodization function and Mertz phase correction. Spectra of micro-sampled cross sections were acquired in transmission mode for 256 scans on a diamond slide.

\subsection{Determining barrier properties from electrochemical data}

From an electrochemical point of view, an ideal protective coating acts like a single capacitor at all frequencies measured. When a coating starts to fail, channels for electrolyte/corrosives open up that act like resistors. In impedance spectroscopy, the polarizability of a coating (capacitance) and barrier to the flow of charge through the material (resistance) is monitored over a range of frequencies. The logarithmic impedance of a capacitor is frequency dependent and represented by a straight line with a slope of -1 while the impedance of a resistor is frequency-independent and represented by a straight line with a slope of 0 .

The total impedance at low frequencies $\left(|\mathrm{Z}|_{0.1 \mathrm{~Hz}}\right)$ has been used to provide a quantitative value for comparison across coatings, where in an overly simplistic interpretation the larger the value, the better the protective quality of the coating. However, this metric neglects contributions from other factors to the impedance. For example, corrosion layers located at the coating-metal interface also dominate the 
spectrum at low frequencies, contributing to raise the $|\mathrm{Z}|$ value. Total impedance values of the best-performing plain bronze sample of each material investigated in this study are plotted during accelerated weathering in Figure 5.1. These data show waterborne PVDF-acrylic clear coatings Kynar Aquatec ARC and FMA (ARC or FMA) have at minimum twice the protective lifetime of solvent-based acrylic Paraloid B-44 (B44),

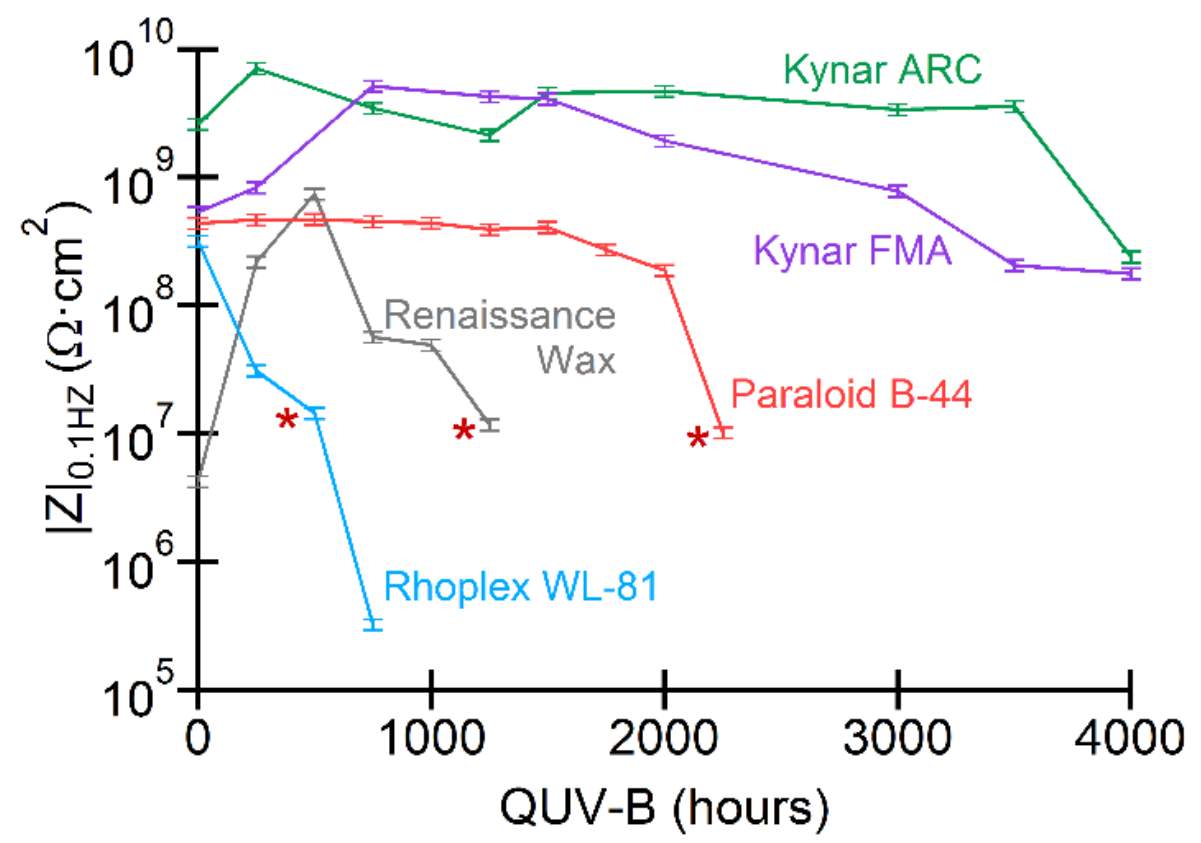

Figure 5.1 An overview of long-term barrier properties of polymeric coatings on plain bronze used in this study. Total impedance at $0.1 \mathrm{~Hz}$ was measured over 4000 hours of artificial OUV-B weathering of the panels, where the asterisk $\left(^{*}\right)$ marks macroscopic film failure by cracking and was accompanied by a decrease in barrier properties. The Kynar ARC and FMA coated panels were both annealed before weathering.

four times that of microcrystalline Renaissance wax, and eight times that of waterborne acrylic Rhoplex WL-81 (WL81). Although the overall trends of this parameter were consistent with the protective lifetimes discussed later in this work, a great deal of pertinent information is missing. Most importantly this includes darkening of substrates coated with wax and B44 approximately 500 hours before the coatings fail due to 
increased porosity of the coatings. The tarnished appearance indicates a passivation layer developed on the surface of the metal, offering temporary protection, but is both aesthetically unpleasing and can lead to irreversible damage of the underlying metal substrate.

EIS data of coatings are often fit to equivalent electric circuits (EEC) to model the physical state of the system (which includes the coating, substrate, defects and the corrosion layer) during weathering. However insightful, circuit model fitting is a timeintensive data analysis method and thus it is impractical for large numbers of samples. To rapidly diagnose the protective quality of coatings and detect defects, we have employed recently reported faster data analysis method developed in our lab. ${ }^{158}$ In this method we use the minimum impedance value of the first derivative $\left(k^{\prime}\right)$ of the impedance spectrum as a metric for comparison across coatings. For clarity, the minimum of $k^{\prime}$ is represented by its absolute value $\left|k^{\prime}\right|$ with units normalized to the working area of the sample measured $\left(\Omega \cdot \mathrm{cm}^{2}\right)$. Coatings were considered no longer protective when the $\left|\mathrm{k}^{\prime}\right|$ value was less than $10 \mathrm{M} \Omega \cdot \mathrm{cm}^{2}$.

This method of analysis is particularly sensitive to low frequency measurements since they have the highest impedance. This is where pores and pinhole defects appear in the EIS spectrum as they provide shortcuts for corrosive species to the metal working electrode. A decrease in $\left|k^{\prime}\right|$ is usually related to polymer degradation and could be due to: (1) increased pore formation, (2) decreased film thickness or (3) mechanical disruption of the film from oxide growth at the coating-metal interface. More complicated, an increase in $\left|k^{\prime}\right|$ indicates an improvement of barrier properties due to: 
(1) formation of a passivating oxide layer at the bottom of pore channels or (2) the coating-metal surface, and in the case of waterborne resins, (3) increased close packing and inter-particle diffusion of latex particles. Therefore, a low $\left|k^{\prime}\right|$ ranking score does not necessitate bulk failure of a film and likewise a high $\left|k^{\prime}\right|$ score can result from corrosion present at the metal-coating interface. While the $\left|k^{\prime}\right|$ values are affected by numerous factors, decreases from their initial or maximum values indicate that the coating is compromised.

The discussion of protective coatings on steel is limited to weathering methods by QUV and Portland, OR due to the aggressive nature of Southern Florida weathering with a salt spray. After six months, only samples with Incralac, B44, and Kynar ARC or FMA were still intact enough to take gloss measurements but not perform EIS. A summary of protective lifetimes discussed in the following sections in addition to gloss and thickness retention values is included in Tables 5.2 and 5.3. Pictures of each coating before and during weathering are included in Appendix E, Figures E7-14.

\subsection{Conservator blind study}

Coatings having high initial impedances, including Incralac and waterborne fluoropolymers, were included in a blind study sent to conservators across the US for evaluation of aesthetic and workability properties. Each participant was provided with a kit containing the same type of brushes, gloves, stir sticks, cloth, and explicit instructions for application of each coating (labeled $\mathrm{CO}_{1}, \mathrm{CO}_{2}$, etc.) onto patinated bronze and steel substrates. After coating, each participant completed an exit survey. 


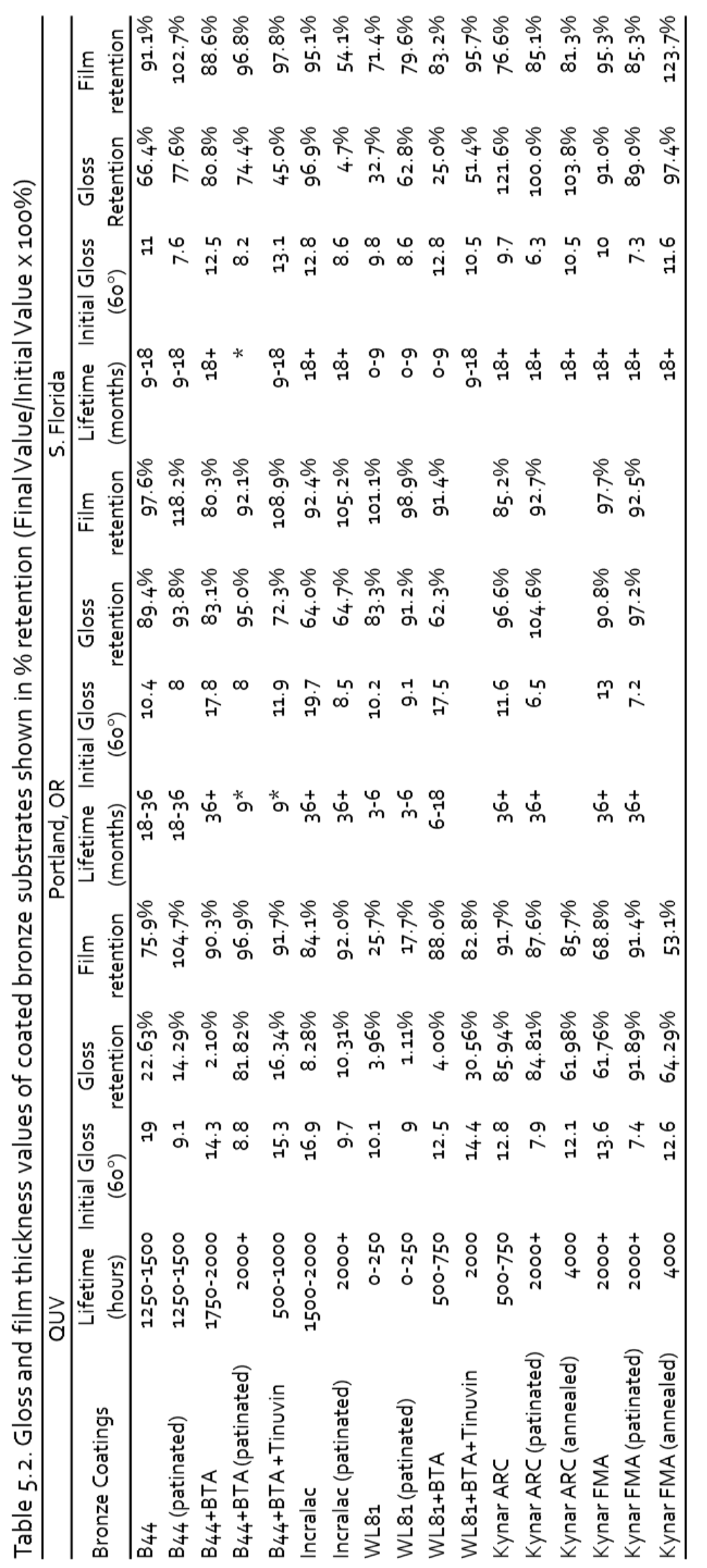




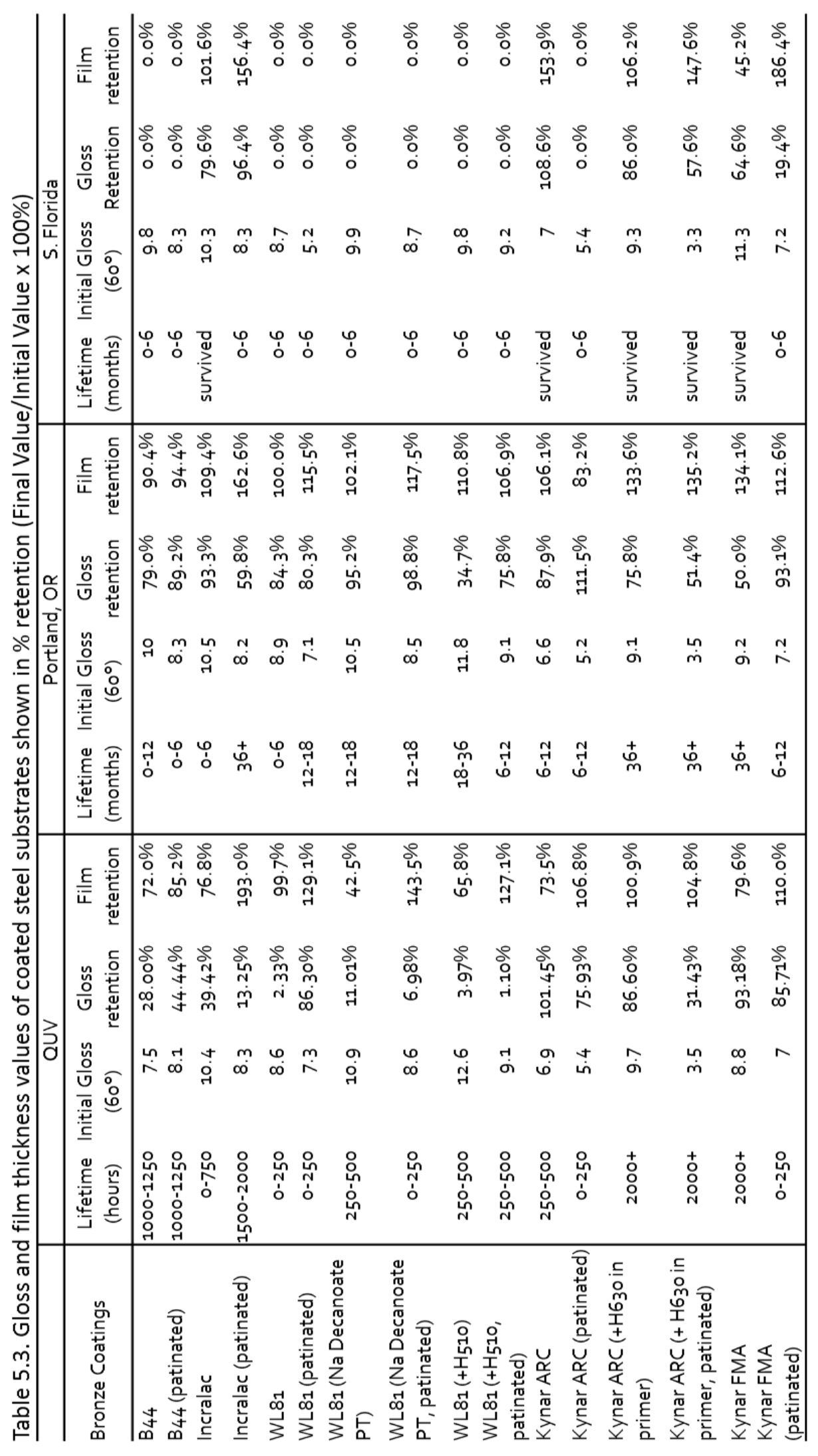


Results of the survey are shown in Figure 5.2. All of the participants regularly work with substrates of copper alloys while only half regularly work with iron alloys. Participants had varying levels of prior experience with different coatings, with most reporting having used waxes and solvent-based acrylic lacquers. $92 \%$ of participants rated workability, clarity, and gloss as "Important" or "Extremely Important" factors when choosing a protective coating. When surveyed about coating selection conservators responded that they were more likely to use an "environmentally friendly" (100\%) coating, that was "reversible" (58\%) with a "long working lifetime" (50\%). Given that only 3 participants had previously used waterborne acrylic emulsions, that all participants indicted that they would be more likely to use an environmentally sustainable coating showed their willingness to try new materials. All panels were sent back to the lab for further analysis of gloss and barrier properties. An analysis of the data from the blind study of waxed panels was previously published [See Chapter o].

All samples were patinated prior to shipment to the conservators. Using a $65^{\circ}$ gloss angle on black glass for reference (100 \%Reflectance), the participants reported acceptable gloss levels as $\leq 6.5 \% \mathrm{R}$, while $6.6 \% \mathrm{R}$ to $7.8 \% \mathrm{R}$ was slightly too glossy and anything greater than $7.9 \% \mathrm{R}$ was too glossy. The average gloss of the B-44 and Incralac panels coated in the study was $7.7 \% R, W L 81=8.7 \%$ and $B 44 / A R C=6.8 \% R$. It is noteworthy that the Kynar ARC is not only less glossy than Incralac, but was also the only coating, aside from wax, that lay within the acceptable gloss range based on the participants' responses. 
When selecting a coating, how important do you rate the following?
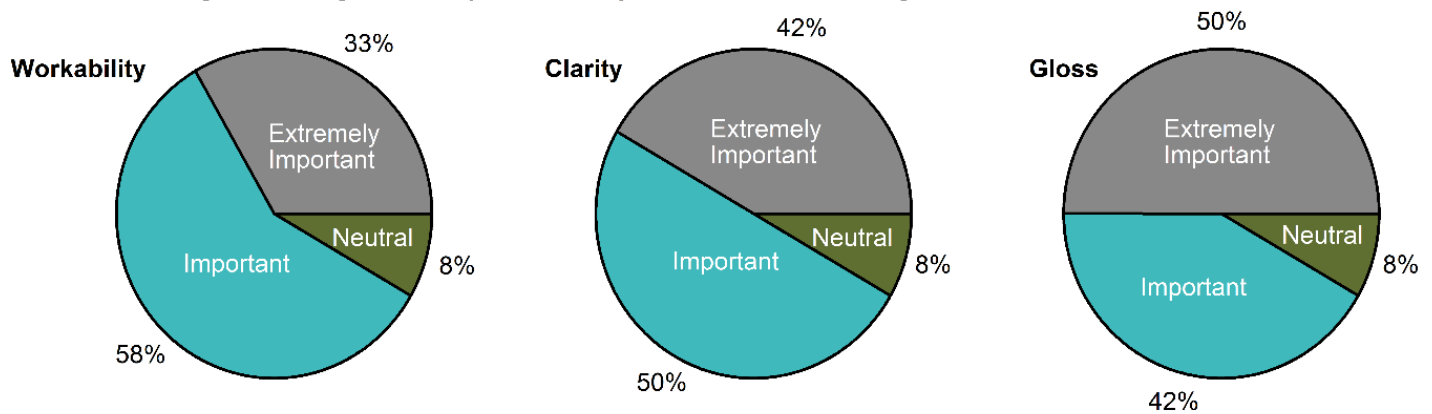

Note: $0 \%$ of responses listed these parametrs as "Not Important"

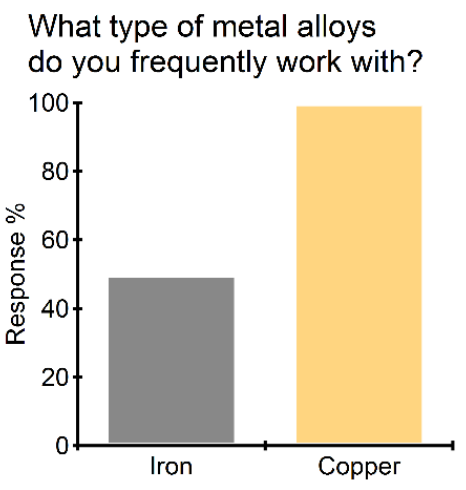

What type of coatings do you routinely use on which type of substrates?

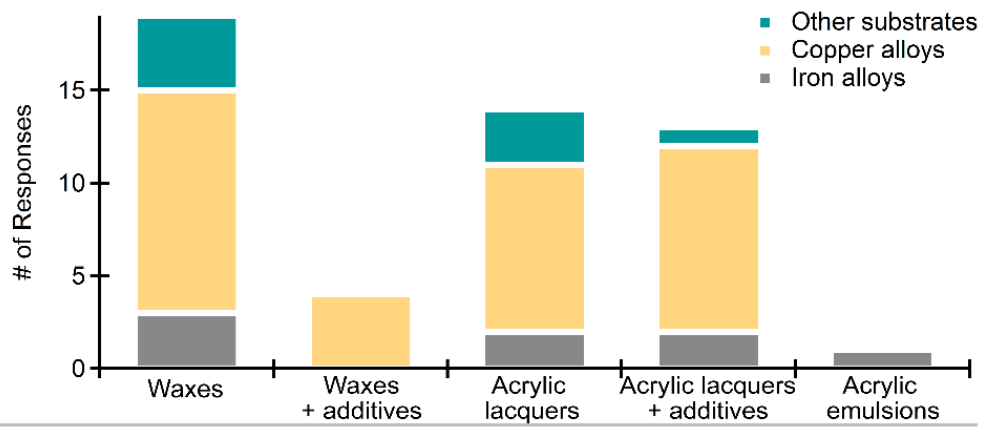

How likely are you to choose a coating with the following labels?
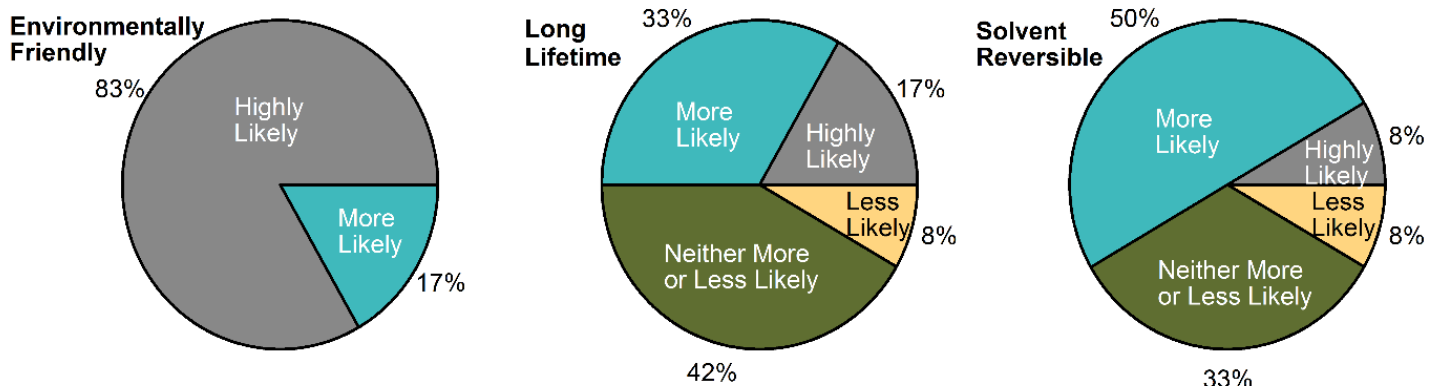

Note: $0 \%$ of responses chose "Never likely" for these labels

Figure 5.2 Results of exit survey from conservators who participated in a blind study of coatings listed in Figure 5.3

Each conservator was supplied with detailed application instructions to ensure consistency across panels. As a control and for comparison, test panels were also produced by the lab. Impedance spectroscopy was performed on a representative area of each panel $\left(15 \mathrm{~cm}^{2}\right)$ to investigate the variability in barrier properties between the 
panels. The averaged impedance spectra from each coating type are shown as Bode plots in Figure 5.3. The dashed and dotted lines are the lab controls (which were not included in the averaged spectrum) and show that each coating formulation can have a high impedance value, even when applied by brush.

The averaged impedances of coated bronze panels at $0.1 \mathrm{~Hz}$ were all between 0.1-1 $\mathrm{G} \Omega \cdot \mathrm{cm}^{2}$, with the highest being waterborne acrylic WL81+BTA, followed by Incralac, and then waterborne PVDF/acrylic Kynar ARC on a B44 primer coat. Averaged impedances of coated steel at $0.1 \mathrm{~Hz}$ were significantly lower than on bronze, and in many cases, were low enough to be classified as being poorly protective. The value of the phase angle was stable and was close to $-90^{\circ}$, indicating that few paths to the metal substrate exist (which, when present are indicated by phase transitions to $0^{\circ}$ ). Breakpoint frequencies are measured at $-45^{\circ}$ and estimate the time charged species require to travel through a film. This parameter was observed to be very short (occurring at high frequencies) in waxes [See Chapter o].The time-constants in Figure 5.3 showed a wide range but ordered from longest to shortest: $0.12 \mathrm{~Hz}$ for Incralac/BrP, $2.2 \mathrm{~Hz}$ for Kynar ARC/B44/BrP, 8.1 Hz for WL81+BTA/BrP, $225 \mathrm{~Hz}$ for B44/StP, $398 \mathrm{~Hz}$ for Kynar $\mathrm{ARC} / \mathrm{B}_{44}+\mathrm{H} 630 / \mathrm{StP}$, and $1976 \mathrm{~Hz}$ for $\mathrm{WL} 81+\mathrm{H}_{510} / \mathrm{StP}$. Of the panels produced by the participants, those coated with Incralac had the highest averaged impedances but also the largest variability among samples. Kynar ARC with a B44 primer also showed good barrier properties and less sample-to-sample variation. 
(a) Solvent-based Acrylic
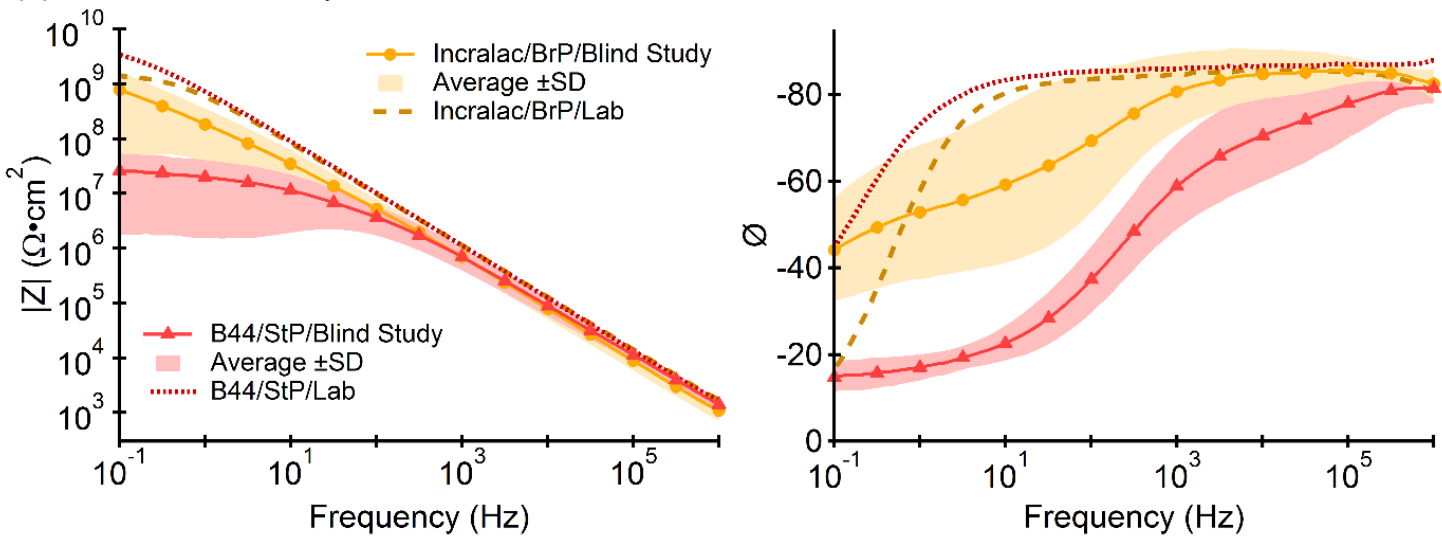

(b) Waterborne Acrylic
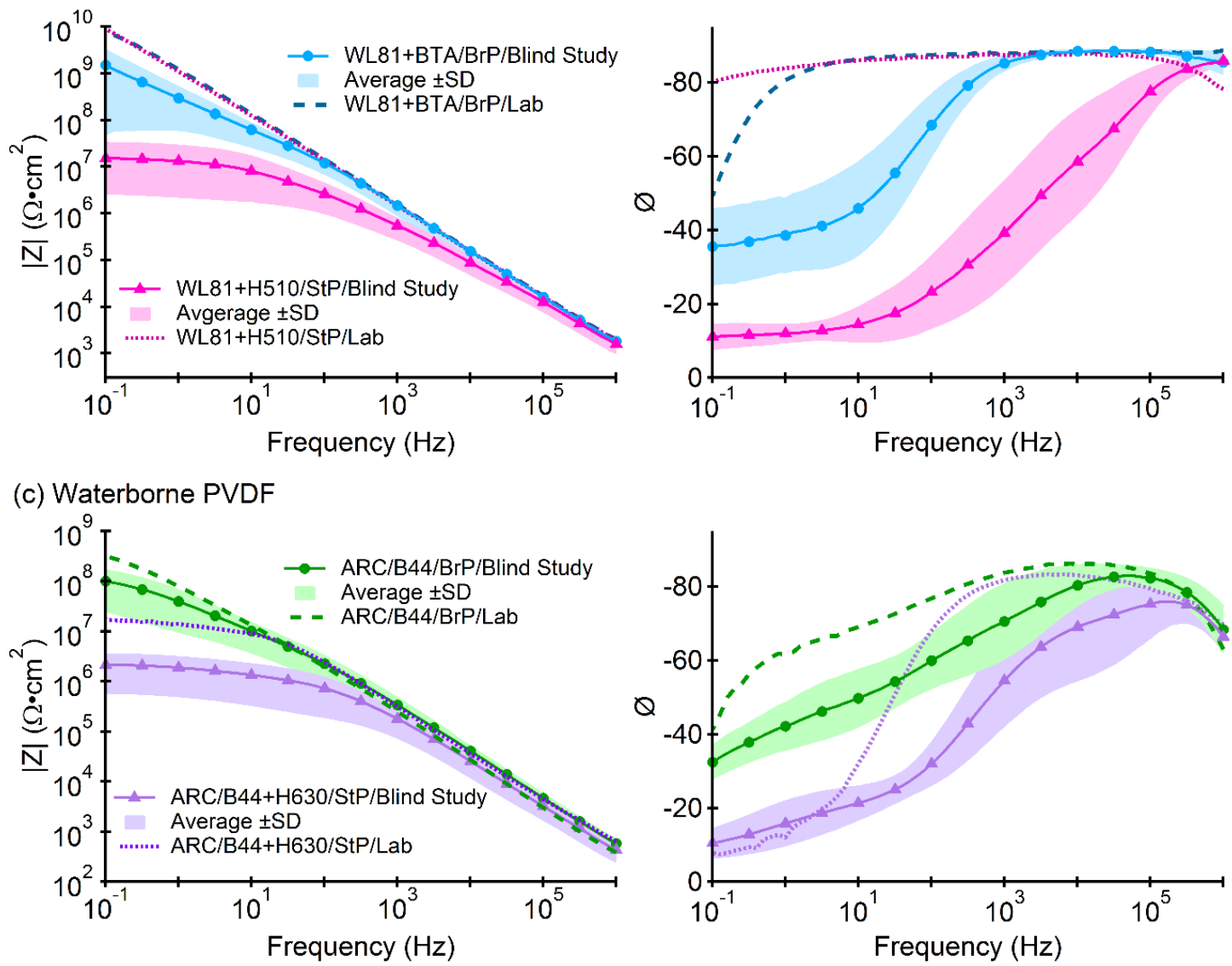

Figure 5.3 Averaged EIS Bode plots of coated bronze and steel samples by blind study participants (markers) ( $n=13$ ) compared with laboratory control samples (broken lines). The shaded regions denote plus and minus one standard deviation from the averaged data. 


\subsection{Solvent-based and waterborne acrylic coatings}

\subsubsection{Barrier properties during weathering}

The rate of change in impedance of $\mathrm{B}_{44}$ coatings is shown in Figure $5.4 \mathrm{a}$, where the protective lifetime for bronze panels weathered outdoors was 18-36 months in Portland, OR and 9-18 months in South Florida. The longest point of failure we have ever observed for B44 on bronze undergoing QUV weathering was 2250 hours, ${ }^{54}$ however the onset of failure for that panel was observable before 2000 hours of weathering and the protective lifetime was more typically between $1250-1500$ hours by QUV as shown in Figures $5.4 a$ (plain) and Appendix E1a. In contrast, plots of steel panels coated with $\mathrm{B}_{44}$ are shown in Appendix E2 and had much longer lifetimes for accelerated weathering by QUV (1000-2000 hours for plain, 1000-1250 hours patinated) than in Portland, OR (o-6 months). No advantage was observed for patinated bronze or steel panels as compared to those without, since the working lifetimes were similar, or worse, for each type of weathering.

Given its long history of use by conservators and detailed discussion in the literature, it was not surprising that bronze panels coated with Incralac had the longest working lifetimes of the acrylic resins in this study (Fig. 5.4b). Only one bronze Incralac coated sample studied here dipped significantly below the minimum protective threshold: QUV weathered plain bronze without a patina whose lifetime for artificial weathering was estimated as $1500-1750$ hours and greater than 2000 hours when patinated. Some pitting and edge corrosion was present on the surface of each Incralac sample but none failed during the course of outdoor weathering. As such the protective 
(a) B44 (plain)

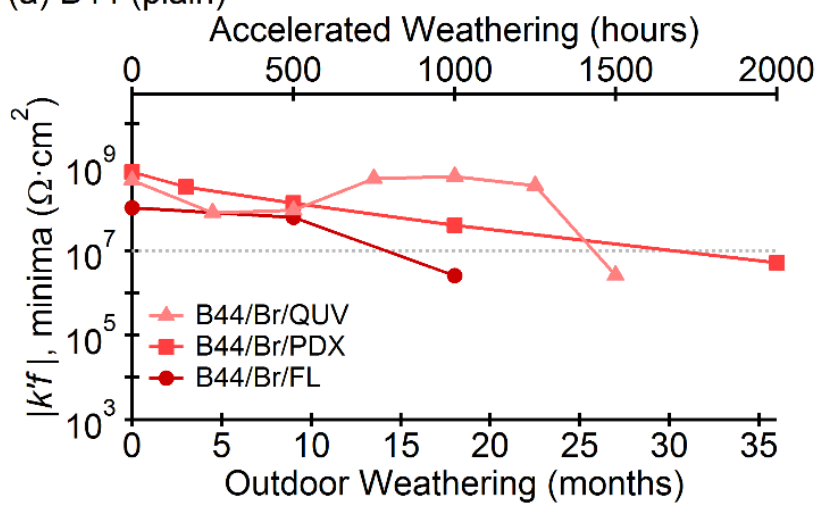

(b) Incralac (plain)

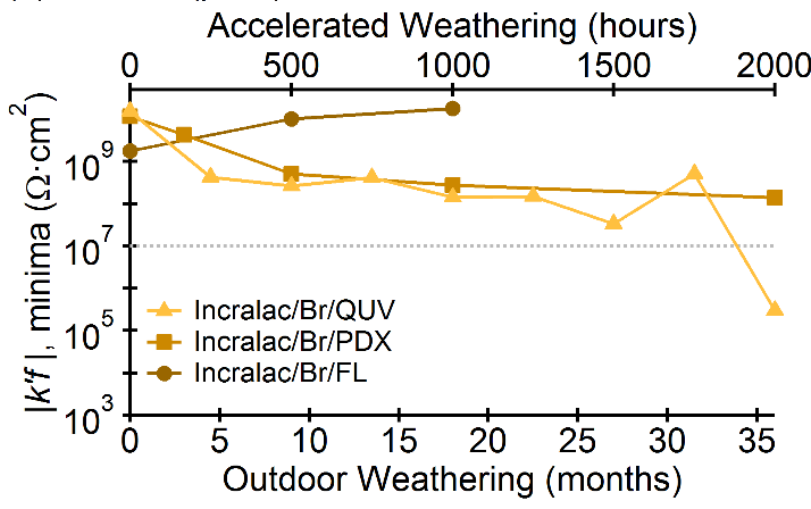

(c) WL81 (plain)

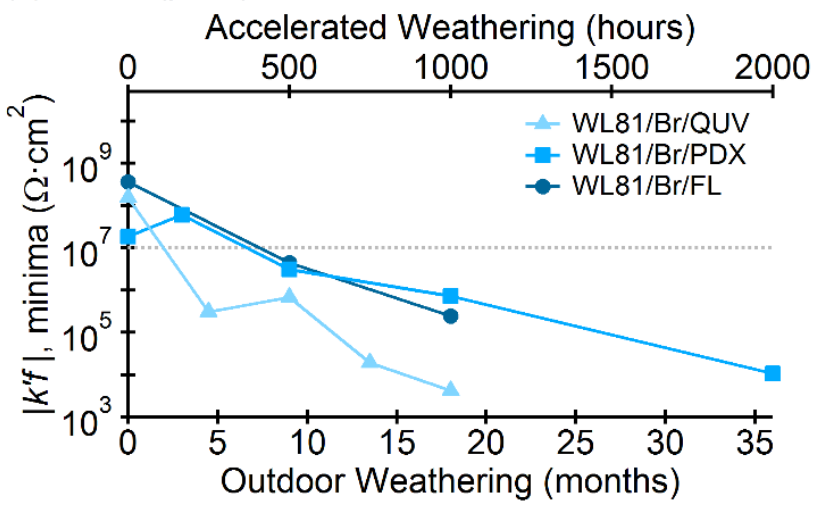

Figure 5.4 Impedance derivatives during weathering of plain bronze panels coated with acrylic resins (a) Paraloid B-44, (b) Incralac, and (c) Rhoplex WL-81.

lifetime for each plain and patinated bronze panel was 36 months in Portland, OR and 18 months in South Florida. The increase in |k'| of the Florida weathered bronze is likely due to filling of pores with corrosion, which was apparent by visual inspection. With similar barrier properties, the only observable difference with the addition of a patina 
on bronze was a small increase in initial performance. Unexpectedly, Incralac performed well on steel panels when patinated but coatings on plain panels were not adequately protective $\left(<10 \mathrm{M} \Omega \cdot \mathrm{cm}^{2}\right)$ before weathering and show large increases in $\left|\mathrm{k}^{\prime}\right|$ from passivating corrosion during exposure. The lifetime of Incralac on patinated steel was about 1500 hours of QUV weathering and 36 months in Portland, although some corrosion is visible on the surface of both panels. For both alloys, the better performance of Incralac with a patina may be due to better adhesion to the substrate. Visually, the samples appeared similar to those of B44: with some pitting from PDX and FL weathering and tarnishing from OUV exposure, all of which was not as readily apparent on patinated panels. Hazing had also developed near the end of the OUV lifetimes, similar to the breakdown of B44 films which eventually lead to cracking. Since the coating has no pigment to create chalking, the opaque film may be due to BTA in the formulation as has been proposed by others. ${ }^{9}$

An effort was made to replace the acrylic B44 coating with a waterborne acrylic latex of comparable chemistry, WL81, which is comprised mostly of poly(n-Butyl methacrylate) with some polystyrene added to increase workability. This resin has good flow during application and repeatedly produced coatings with few defects in the dry film on bronze and was ranked highly workable by conservators in the blind study. Given the capability of this resin to produce high impedance coatings, it was proposed WL81 would be a candidate to out-perform the working lifetime of B44. However, the majority of panels coated with WL81 did not survive long past the first weathering time-point (Fig. 5.4b) and the protective lifetime of this film on plain and patinated 
bronze was found to be 3-6 months in Portland, 0-9 months in South Florida, and 0-250 hours by QUV weathering. On plain and patinated steel substrates in Appendix E2, impedance measurements show these samples were not considered protective at any point during weathering. The increase in $\left|k^{\prime}\right|$ of Portland weathered steel with a patina at 6 months was due to the development of corrosion on the substrate and was not considered protective at that time point. Flash rusting indicated a corrosion inhibitor needed to be added to apply the coating to plain steel, and sodium decanoate was used as a pretreatment to produce an aesthetically pleasing and high-impedance film. With the pretreatment, WL81 had a longer lifetime of 250-500 hours (plain) and 0-250 hours (patinated) by QUV and 12-18 months in Portland (plain and patinated). Overall, a slight improvement was shown with the addition of a patina to WL81 coated bronze and steel, but only in impedance compared to plain samples, not in lifetime. While higher in initial impedance, the barrier properties of WL81 without additives were similarly effective as previously studied wax coatings.

For outdoor weathered solvent-based coatings on bronze or steel, much of the coating was still intact at the end of the assigned lifetime but a number of pits were visually apparent. We have shown EIS is sensitive enough to detect the pinholes frequently present in fresh B-44 coatings, ${ }_{1}^{158}$ even when great care is taken to produce uniform panels in the lab. It can be assumed many such narrow paths to the metal substrate will exist on large coated objects outdoors, and will fill with corrosion to increase the impedance of the film at first (seen here as a sharp increase at one time point and a significant drop at the next). However, this passivation layer at the bottom 
of pores is fleetingly protective and eventually can lead to irreversible and uneven pitting corrosion. The low $\left|\mathrm{k}^{\prime}\right|$ apparent immediately upon outdoor weathering of all acrylic coated steel was due to poor film formation on the steel surface, revealed by a number of pinholes that consistently lead to a low frequency resistor by impedance analysis. Due to the highly active surface of steel (as compared to bronze), these pinholes rapidly turned into large pits that damaged large working areas of the samples.

\subsubsection{Chemical degradation}

A well-known difficulty with solvent-based coatings is that due to their film formation mechanism, they undergo rapid and irreversible failure when the material has become too brittle. This typically occurs from chain-scission by photodegradation, as the products of PMMA and PEA are highly volatile and eventually the shrinkage from material loss will cause cracking. It is of note that significant yellowing was observed and eventual tarnishing of QUV-weathered panels based on B44 resin, including Incralac, which is common for acrylics undergoing UV exposure artificially and outdoors. The comparative susceptibility to photodegradation of each acrylic coatings is most apparent when comparing the relative magnitude of difference spectra (spectrum after weathering minus spectrum before weathering) shown underneath the full FTIR spectra in Figure 5.5. Since infrared absorbance is directly proportional to concentration, positive peaks in the difference spectra indicate an increase and negative peaks indicate a decrease in concentration of a particular functional group. 
The most notable change for both solvent-based and waterborne acrylics was the loss of the pendent acrylic group as evidenced by a tandem decrease in the following peaks: aliphatic C-H stretch from $3000-2800 \mathrm{~cm}^{-1}, \mathrm{C}=\mathrm{O}$ stretch near $1730 \mathrm{~cm}^{-1}$, and C-O stretch at $1150 \mathrm{~cm}^{-1}$.
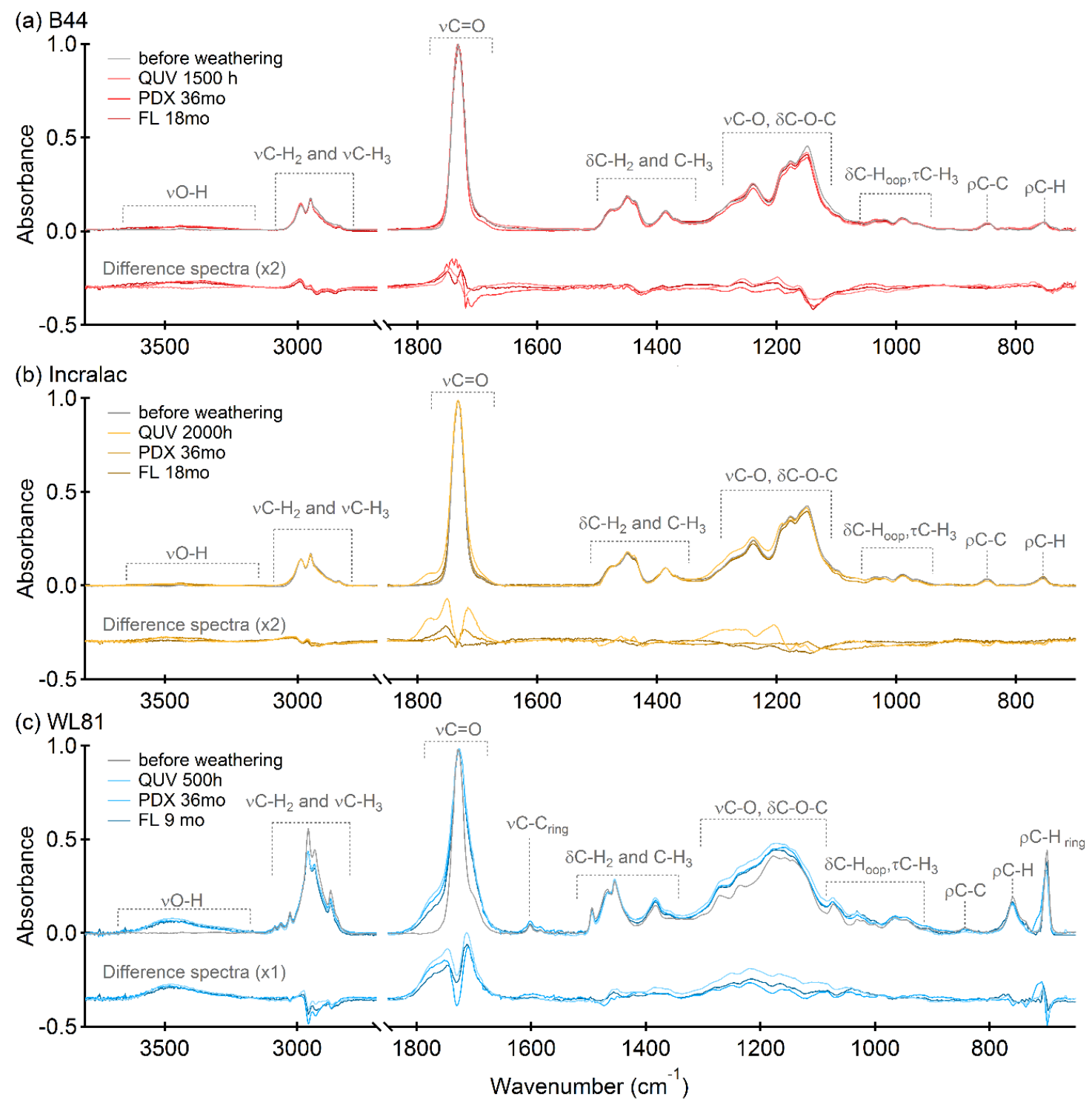

Figure 5.5 FTIR of acrylic coatings on bronze before and after weathering in S. Florida, Portland, OR, and by QUV-B. Spectra were normalized to their respective rocking $\mathrm{C}-\mathrm{H}$ deformation peak.

Very little change was observed by FITR of B 44 (Fig. 5.5a) and Incralac (Fig. 5.5b) at the end of their protective lifetimes, and their difference spectra have been 
magnified (x2) for clarity. Subtraction of the unexposed samples gave better insight into the processes occurring where alongside a few degradation products, a reduction in the methylene peaks coupled with an increase in methyl stretching indicates that extensive chain scission may be occurring. It is suspected that the gradual ablation of material evidenced here spectroscopically gave way to the ultimate mechanical failure of the film, the onset of which was indicated electrochemically in Figure 5.4a. The products of methacrylate polymers by thermal degradation are mostly the relevant monomer occurring through a depolymerization process (methyl methacrylate for PMMA and butyl methacrylate for PnBMA). The products of acrylate polymers (PEA) also include the monomer units through unzipping but are more prone to cleavage of pendent groups that eventually lead to alkenes and long chain alcohols. ${ }^{159}$ The broad shoulder peaks present in all weathered B44 and Incralac spectra near 1762 and 1705 $\mathrm{cm}^{-1}$ are indicative of cyclic anhydrides and carboxylic acids produced from side-chain oxidation.

Infrared spectra of the WL81 film before weathering and after failure showed significant spectral differences (see Fig. 5.5c). While the chemical composition of the WL81 was selected for its good working properties and similar chemical composition to the other acrylic resins used herein, the waterborne resin contained polystyrene (PS) which is well-known to be photochemically sensitive. ${ }^{160}$ Although it has not been documented with these particular polymer systems, acrylic latex emulsions may become more brittle and hard upon aging due to increased cross-linking, and in some cases oxidation, of the dry polymer film. ${ }^{161}$ New peaks in weathered spectra of WL81 
coatings appear at or near 3465 and $3310 \mathrm{~cm}^{-1}$ assigned to $\mathrm{O}-\mathrm{H}$ stretching vibrations and shoulder peaks at 1764 and $1713 \mathrm{~cm}^{-1}$ attributed to the production of carboxylic acids and associated derivatives in the film. A slight decrease in aliphatic $\mathrm{C}-\mathrm{H}_{2}$ stretching groups located from $2874-2959 \mathrm{~cm}^{-1}$ also infers chain-scission like degradation (freeradical induced) with the removal of monomer units from the polymer backbone before oxidation to acid groups.

Such formation of degradation products are readily apparent in the subtraction spectra of the coatings with WL81 as a component, where a 'build-up' of peaks associated with the $\mathrm{C}=\mathrm{O}$ stretching peaks occur at frequencies higher and lower than the target ester of the side groups. After only 500 hours of exposure, the new hydroxyl stretching vibration at $3309.8 \mathrm{~cm}^{-1}$ coupled with the shifting and broadening of the carbonyl at $1727 \mathrm{~cm}^{-1}$ is directly associated with the presence of and broadening of acid functionality in the film, some species of which can dehydrate to give six-membered cyclic anhydride structures as seen in OUV weathered Incralac. ${ }^{162-163}$ Subtraction spectra of the WL81 containing films show evidence of oxidation as well as cleavage of the ester functionality similar to that of B44 and Incralac. The hydroxyl stretching band at $3464.9 \mathrm{~cm}^{-1}$ is associated with hydroxyl groups bound to the cross-linked backbone. ${ }^{164}$ Degradation of polystyrene is typically observed by the release of volatile alkyl benzenes or unzipping of monomer units, which was noted in the IR spectra of WL81 as a decrease in vibrational bands at 2934 and $2875 \mathrm{~cm}^{-1}\left(v_{\text {as }}\right.$ and $v_{s}$ of $\left.\mathrm{C}-\mathrm{H}_{2}\right)$ as well as $1450 \mathrm{~cm}^{-1}\left(-\mathrm{CH}_{2}\right.$ deformation bend in benzene). ${ }^{163,165}$ 
It has been well established that polymers with bulky side groups (e.g. styrene, nBMA, as well as un-methacrylated polymers) are more susceptible to photo-oxidative degradation, producing more volatile products through side group cleavage producing alcohols, carboxylic acids and cyclic esters depending on the length of exposure to radiation. ${ }^{41,143,150}$ The overlap between the outdoor and artificial weathering methods lies primarily in UV exposure, indicating the primary mechanism for degradation of acrylic coatings in this study was photochemical. Only the magnitude of change was different across weathering, most likely related to the level of irradiation and the ratio of UV-A/UV-B received by the samples.

\subsubsection{The effect of additives}

\subsubsection{BTA and Tinuvn}

Given the unexpected and rapid failure of WL81, the composition of the proprietary acrylic resin was investigated further and discovered to contain a small amount of polystyrene. To minimize susceptibility of some acrylics and styrene to photodegradation observed by FTIR in Figure 5.5 , a corrosion inhibitor (BTA) alone and in combination with a light stabilizer (Tinuvin) was added to B44 and WL81 to increase the protective lifetime of the coatings. Tinuvin is a hindered-amine light stabilizer (HALS) that abstracts free radicals from UV radiation before they propagate through a polymer film, and, along with BTA is present in the historic formulation of Incralac. It was expected these additives would increase the lifetime of solvent-based and 
waterborne acrylics since both showed a particular sensitivity to photodegradation and their effects were investigated on plain bronze shown in Figure 5.6.

(a) B44 with Benzotriazole

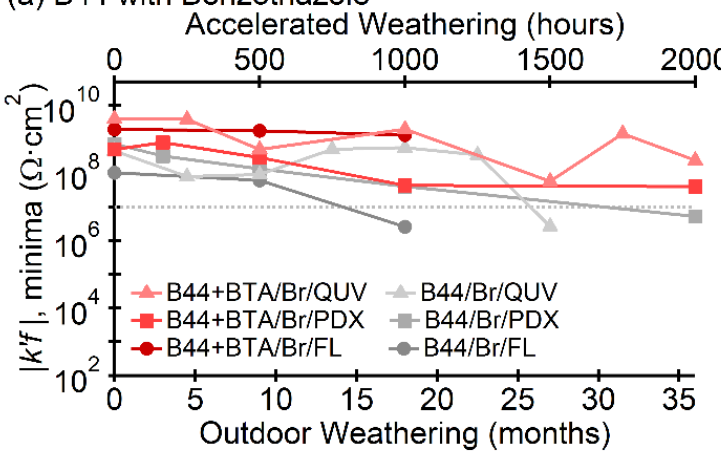

(c) WL81 with Benzotriazole

Accelerated Weathering (hours)

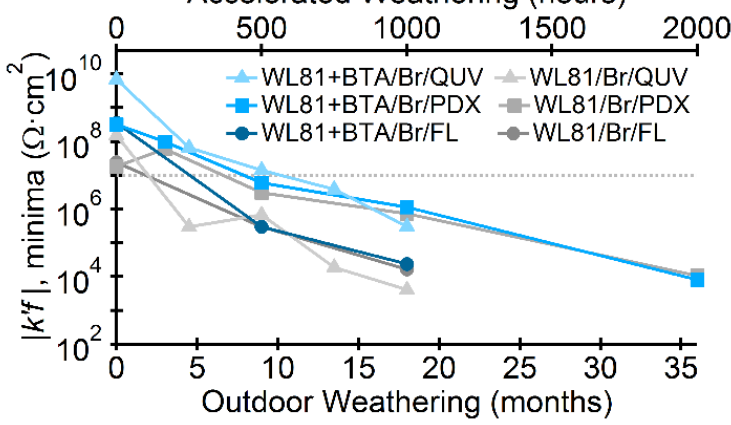

(b) B44 with Benzotriazole and Tinuvin

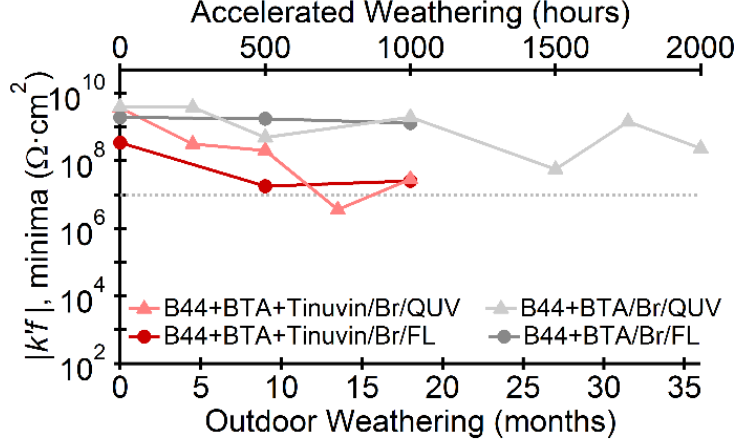

(d) WL81 with Benzotriazole and Tinuvin Accelerated Weathering (hours)

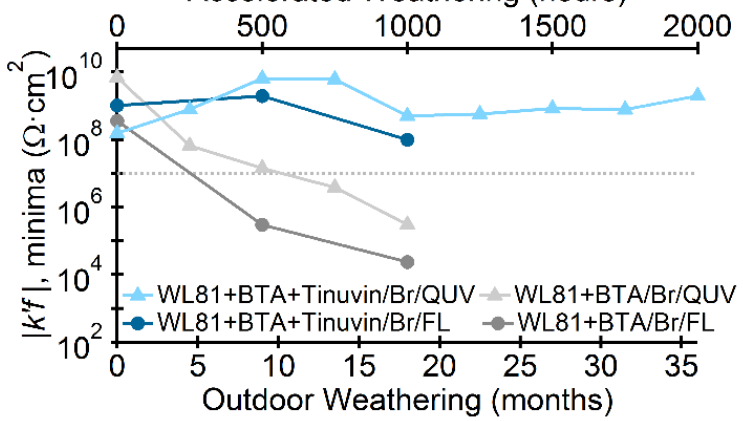

Figure 5.6 Derivative analysis of impedance to investigate the effects of additives BTA and Tinuvin on the barrier properties of WL-81 and B-44 on bronze panel.

Upon the addition of just BTA, an improvement in initial barrier properties and lifetime of both coatings was observed, though the effect was much smaller in WL81 (Fig. 5.6). Plain bronze coated with B44+BTA were considered protective for 36 months in Portland, 18 months in S. Florida, and 2000 hours of QUV weathering. Coatings of WL81+BTA were protective for 3-9 months in Portland, 0-9 months in S. Florida, and 500-750 hours of QUV weathering. In contrast, the addition of BTA and Tinuvin significantly increased the lifetime of WL81 to 18 months in S. Florida and up to 2000 hours by QUV (Fig. 5.6c). The inclusion of Tinuvin in B-44 was not effective and appeared to destabilize the coating by decreasing the lifetime by half to 9-18 months in 
Florida and 500-1000 hours of QUV cycling. Both samples weathered in Portland, OR with Tinuvin added did not survive the first freeze/thaw cycle outdoors, a phenomenon only observed for formulations containing HALS. The visual appearance of these panels with Tinuvin was generally unacceptable, implying too much of the HALS had been added and would not be recommended as a protective coating for cultural works without further optimization and testing of the formulation. Similar to Tinuvin, a commercial light stabilizer Halox 510 was used for steel panels (Appendix E3) where it was observed to increase the overall protection and lifetime of plain steel beyond that seen by pretreatment to $250-500$ hours by QUV and 6-12 months in Portland, and for patinated steel to $500-750$ hours by QUV and 18-36 months in Portland. It is of note that plain and patinated steel panels coated with WL81+Halox 510 did not require a pretreatment of any kind for direct application to the substrates.

It was assumed the longer lifetime of Incralac over just B44 was due to the addition of mostly a corrosion inhibitor (BTA) and UV stabilizer (Tinuvin) which could combat the early onset of corrosion and degradation. Often overlooked, the formulation of Incralac also includes epoxidized soybean oil (EPSO) as a plasticizer, however EPSO can also act as a proton scavenger and is frequently used to neutralize acids produced by the degradation of polymers. ${ }^{9}$ Since this work typically shows failure of B44 from cracking, which was exaggerated by the inclusion of Tinuvin, the EPSO may play an important role in maintaining flexibility of the coating during stress induced by weathering and particularly during extreme temperature fluctuations. Since WL81 has a MFFT lower than the TG of B44, the greater flexibility of the latex may 
reduce the impact of stress induced by Tinuvin that more brittle solvent-based resins cannot do.

\subsection{Fluorinated latex coatings}

\subsubsection{Barrier Properties during weathering}

The initial barrier properties of ARC and FMA top coats over B44 primer coats were lower than expected, given extremely long lifetimes documented in other studies of the same polymers but in solvent-based formulations. ${ }^{166}$ Results in this study showed an increase in $\left|k^{\prime}\right|$, or film resistance, in solvent-based resins occurring from pore-filling corrosion if it was followed by a dramatic decrease in $\left|\mathrm{k}^{\prime}\right|$ and was confirmed by visual inspection at later time points. However for the PVDF films, the initial increase in |k'| for many of the Kynar coatings shown in Figure 5.7a,c (plain) and Appendix $\mathrm{E}_{4}$ (patinated) were sustained throughout weathering and did not correspond to any visible corrosion, except for one sample. We have previously observed, described and attributed similar behavior in waterborne coatings to be the result of incomplete film formation that improved slightly with annealing and dramatically upon QUV exposure, and thus it is likely the PVDF containing topcoats completed film-forming during all three types of weathering. ${ }^{37}$

The plain bronze panel coated with Kynar ARC and weathered by QUV did not show the same aforementioned increase in $\left|k^{\prime}\right|$ as the outdoor weathered coatings and all of the FMA coatings. Visual inspection after 250 hours of QUV weathering showed darkening of the exposed portion of this sample, which was considered 'failed' at 750 
(a) Kynar ARC (plain)

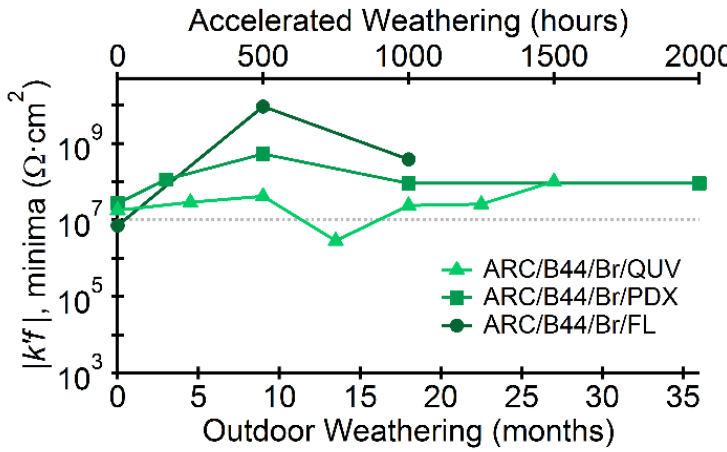

(c) Kynar FMA (plain)

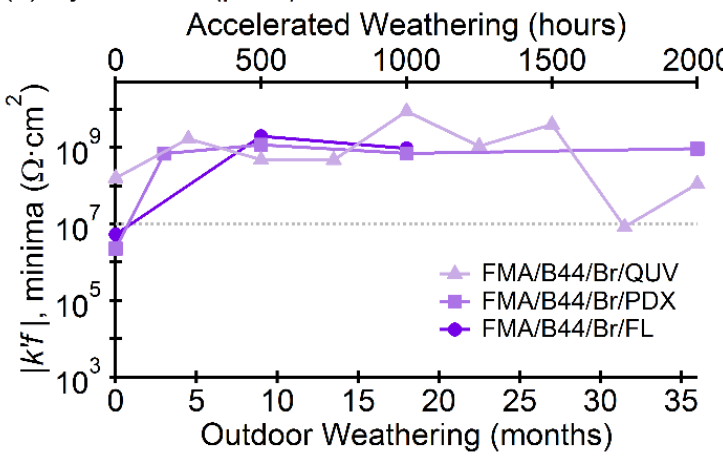

(b) Kynar ARC (plain, annealed)

Accelerated Weathering (hours)

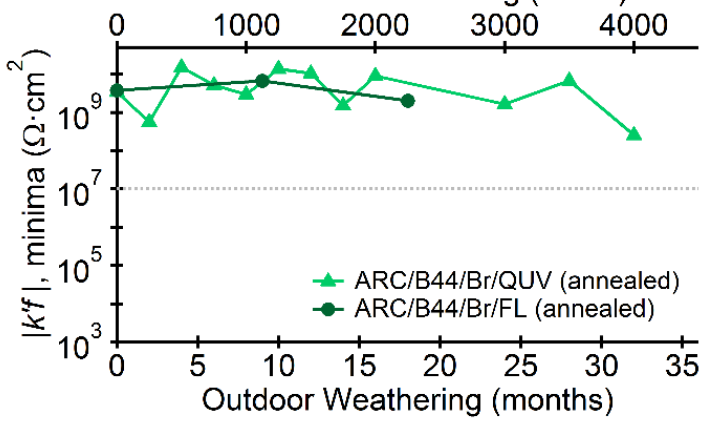

(d) Kynar FMA (plain, annealed)

Accelerated Weathering (hours)

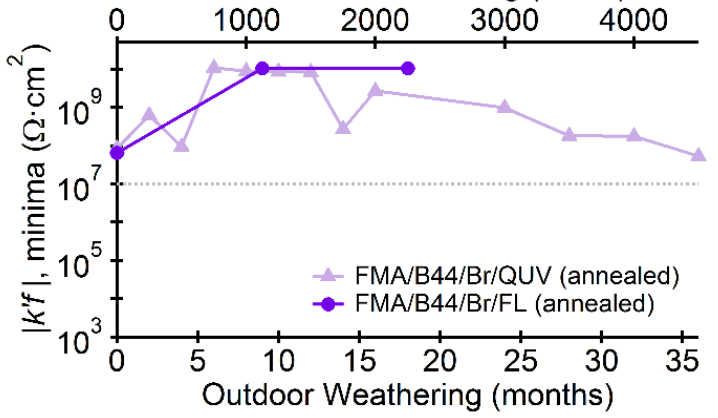

Figure 5.7 Derivative analysis of impedance for Kynar ARC and FMA on bronze.

hours from extensive tarnishing. Given the slight permeability of these films, it is certainly possible that a small amount of corrosion built up at the coating metal interface upon initial exposure. In comparison, the plain bronze panel coated with Kynar FMA had a protective lifetime in this study of 2000 hours and showed no visible corrosion on the surface. All of the plain and patinated bronze panels coated with Kynar ARC or FMA were considered protective for the entire study of 36 months in Portland and 18 months in S. Florida. It appears that extended coalescence outdoors under less intense conditions than QUV 'healed' any remaining defects, but these points would still be susceptible to mechanical failure after extensive film loss/strain from weathering. A few oscillations in $\left|\mathrm{k}^{\prime}\right|$ are apparent for samples in Figure 5.7 and small spots of corrosion were visible in a few samples at 36 months of Portland weathering, 
18 months in Florida and 2000 hours of QUV. Patinated bronze coated with these waterborne PVDF resins (Appendix E4) showed less fluctuation in $\left|k^{\prime}\right|$ and larger impedance overall as compared to plain bronze. We suspect that any pore filling corrosion growing from the artificial patina would be more stable than from the bare metal, and the latter could mechanically disrupt the film at the coating metal interface by delamination.

In previous work we showed that residual additives and coalescing agents remained in PVDF/acrylic latex systems after application and extended drying time or annealing treatments were necessary to prevent ion migration through the coating. ${ }^{26 \text {, }}$ ${ }^{37,89}$ To improve barrier properties of the PVDF resins to their known capabilities, the coatings were annealed before weathering and the results for plain bronze are shown in Figure $5.7 \mathrm{~b}, \mathrm{~d}$. Here the protective lifetimes of both ARC and FMA were 18 months in Florida and 4000 hours by QUV. The same improvement of initial impedance upon annealing was not observed in the FMA coatings as compared to ARC and an explanation may lie in the difference of MFFT of each resin $\left(12^{\circ} \mathrm{C}\right.$ for FMA versus $27^{\circ} \mathrm{C}$ for ARC). The ARC resin is 70\% PVDF:30\% Acrylic and PVDF is well-known to retain some crystallinity that would decrease the mobility of the polymer and reduce deformation of the latex particles during coalescence - leading to possible film formation defects. Conversely, the FMA resin is 50\% PVDF:50\% Acrylic and has a MFFT below room temperature. The annealing effects here may be twofold: (1) driving off residual solvent of films to reduce water sensitivity, observed for both ARC and FMA, and (2) increased film coalescence to improve polymer network integrity, observed only 
for ARC. For both bronze and steel samples without a patina, visual inspection of the Kynar coatings at the end of their QUV lifetime (up to 4000 hours for annealed films) showed significantly less corrosion on the surface of the FMA sample (small amounts of pinhole corrosion) as compared to the ARC. It is also of note that the visual appearance of 15 out of the 16 PVDF coated bronze samples was far superior to that of the B44 or Incralac at the end of the weathering study.

Given the early time of failure and inert chemistry expected of the ARC resin, the plain bronze sample weathered by QUV was suspected to fail from increased water sensitivity as opposed to polymer degradation seen in the acrylics. Similar to results for bronze, the FMA resin out-performed ARC coatings on plain steel (Appendix E5) where the protective lifetimes were 36 months in Portland and 2000 hours of QUV for FMA and $0-6$ months in Portland and 0-500 hours of QUV for ARC. Both PVDF coatings on patinated steel had a lifetime of 6-12 months in Portland and 0-250 hours by QUV. Previous work shows coalescent migration from the PVDF topcoat to the $\mathrm{B} 44$ base coat which can soften the base coat and increase hydrophilicity of the entire film to allow for greater electrolyte uptake and the early-onset of corrosion. We deem this phenomenon responsible for the early onset of corrosion and poor performance of the ARC resins on steel. A commercially available corrosion inhibitor was added to the B44 primer, to reduce the impact of this phenomenon while the coating continues to coalesce (up to months). Samples with this reformulation are shown in Appendix E6 and led to an increase in overall barrier properties as well as an increase in protective lifetime for both plain and patinated steel to 36 months in Portland and 2000 hours by QUV. 
Furthermore, although EIS measurements could not be obtained, this coating was one of the only steel samples to 'survive' Florida weathering.

\subsubsection{Chemical degradation}

FT-IR spectral analysis in Figure 5.8 indicated little significant chemical change in the multi-layered system with a KYNAR ARC topcoat after 2000 hours QUV-B weathering. For confirmation, spectral subtraction before and after exposure showed only minor detectable chemical changes in the polymer film, where no indication of known degradation products was found but spectroscopic evidence indicated some loss of material in the acrylic component of the coating. A general decrease in principal polymer peaks (aliphatic $\mathrm{C}-\mathrm{H}, \mathrm{C}=\mathrm{O}$ stretching, and $\mathrm{C}-\mathrm{O}$ stretching vibrations) occurred in each coating. Much like the $\mathrm{CH}_{2}-\mathrm{CF}_{2}$ unit in fluorinated resins, the methyl group substituted into the backbone of PMMA (the primary copolymer unit in $\mathrm{B} 44$ and the Kynar resins) is thought to inhibit the free radical transfer process associated with the photodegradation of polymer systems and is touted as the main component responsible for the UV-inertness of the resin. ${ }^{42,151,153,155-156}$ This would necessitate that any type of chemical change leading to the degeneration of these films occurred as side-group cleavage or monomer 'un-zipping' as opposed to cross-linking, cyclization and/or mid-chain scission. Support for these claims can be found with the small changes seen in films with $\mathrm{B} 44$ as a component and a complete lack of identifiable degradation products in the Kynar resins. Products formed by side-group cleavage or unzipping would likely be volatile in nature and wash out of the film during weathering, 

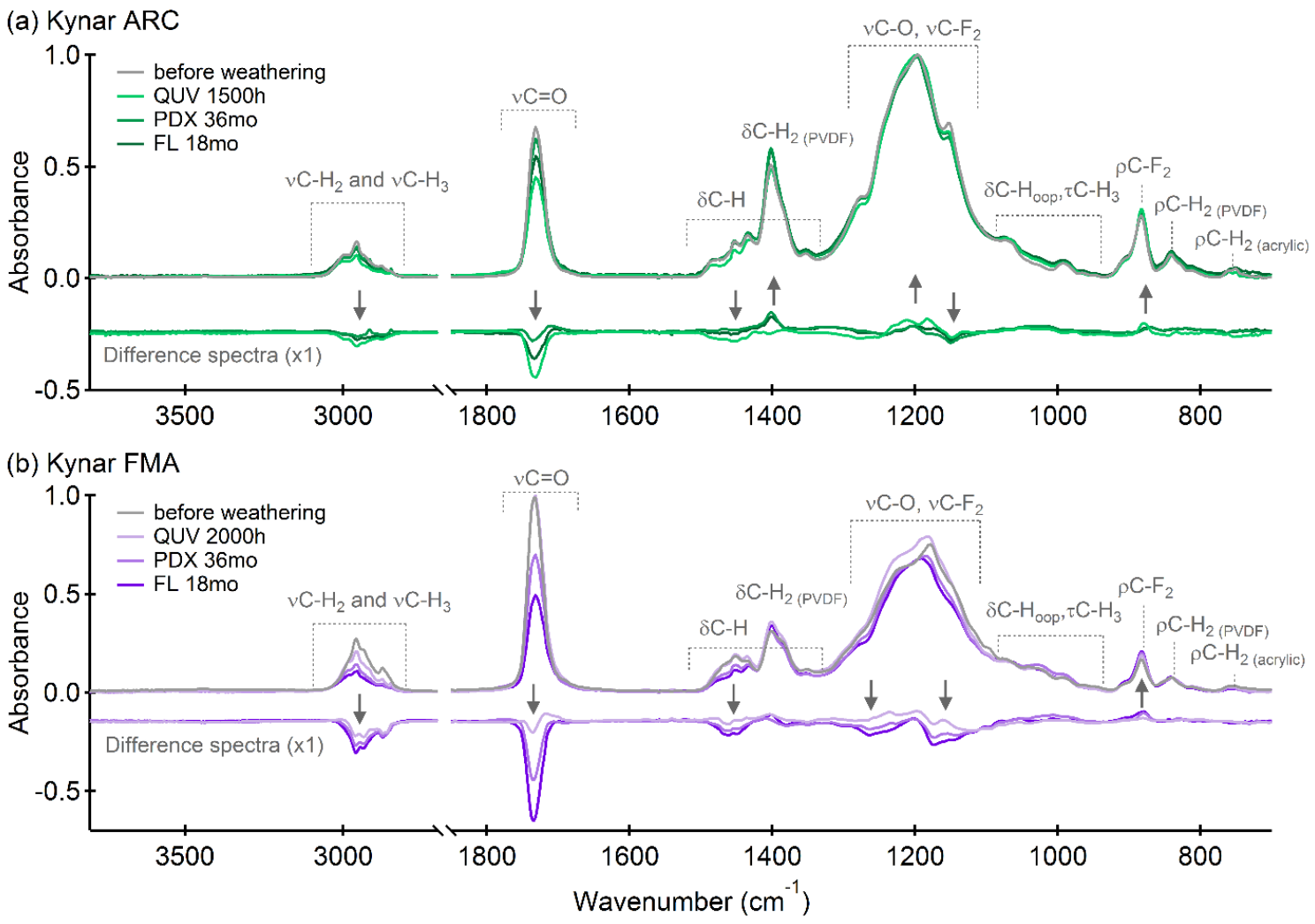

Figure 5.8 FTIR of acrylic coatings on bronze before and after weathering in S. Florida, Portland, OR, and by QUV-B. Spectra were normalized to their respective rocking C-H2 (PVDF) deformation peak.

as opposed to cross-linked and oxidation products (e.g. cyclic esters) which are not usually volatile and may change the properties of the polymer/coating.

Additionally, each of the PVDF/Acrylic blends has a readily identifiable carbonyl peak apart from the various acrylate ester copolymers that may change upon exposure due to oxidation of "unzipped" monomer units. These copolymer blends also have distinct vibration bands occurring near $881 \mathrm{~cm}^{-1}$ that are characteristic peaks assigned to $\mathrm{CF}_{2}$ stretching and $\mathrm{CH}_{2}$ twisting of PVDF. ${ }^{11,42}$ Relative decreases of the acrylic peaks as compared to those relating to PVDF in the IR specrtum of B44/ARC provide evidence that degradation of the polymer is occuring in the acrylic component of the resin and not in the PVDF portion. This suggests that PVDF not only had longer protective 
lifetimes but was indeed more photochemically stable during weathering than any of the acrylic-only films tested. Surface analysis by ATR [see Section 6.6] showed no trace of the $\mathrm{B}_{44}$ basecoat in the infrared spectrum from the ARC coatings but a nearly complete loss of acrylic functionality in the top coat. These results were in line with observations by others ${ }^{42,166}$ that indicate the acrylic portion of the blend is sacrificial and instead of surface erosion (ablation) as seen in the acrylic-only coatings, the fluorinated blend contracts to produce surface enrichment of PVDF. The degradation of these coatings was seen to slow down over time by FTIR, amd as such it is likely that surface enrichment of PVDF slows down the degradation of any remaining acrylic under the surface as well as the acrylic-only primer coat.

\subsection{Physical properties after weathering}

Evidence from other methods has indicated that $\mathrm{B}_{44}$ forms higher molecular weight fragments before undergoing chain scission processes. ${ }^{148}$ Samples weathered by accelerated QUV were compared to fresh cast resins since they were exposed to the highest level of UV irradiance, and would subsequently have the highest abundance of photodegraded polymer. A decrease in Tg or MFFT is indicative of smaller molecular weight chain fragments produced from either mid-chain and/or side-chain scission. Conversely an increase in Tg or MFFT typically occurs from cross-linking which increases the molecular weight and slows down molecular motion of polymer chains. The initial Tg of fresh B44 (Fig. 5.9a) was confirmed at $60.7^{\circ} \mathrm{C}$ and upon weathering two main populations of the resin appeared with midpoint values at $30.8^{\circ} \mathrm{C}$ and $64.9^{\circ} \mathrm{C}$ and 
confirmed both chain-scission and cross-linking occur in the B44 coatings subjected to accelerated weathering. Interestingly, a fresh recast of WL81 (Fig. 5.9b) shows an MFFT lower than the manufacturer's value at $27.8{ }^{\circ} \mathrm{C}$ with an increase to $58.7^{\circ} \mathrm{C}$ upon weathering. Two more populations, much smaller in number were also seen at $40.6^{\circ} \mathrm{C}$ and $83.3^{\circ} \mathrm{C}$, indicating that many degradation processes are occurring in WL81, including both chain-scission and cross-linking, as was evidenced by FTIR. The MFFT of Kynar ARC (Fig. 5.9C) was found to be $23.8^{\circ} \mathrm{C}$, slightly below the published value, with an increase to $40.2{ }^{\circ} \mathrm{C}$ upon QUV weathering. The increase here is also likely due to some cross-linking and decreased mobility of the acrylic component of the resin. Extensive side-chain cleavage can eventually lead to cross-linking and recombination of the polymer back bone. Good gloss and thickness retention are also important parameters of coating weatherability and these values are listed in Tables 5.2 and 5.3 for all coated samples in this study. The highest gloss retention was observed for bronze coated with the PVDF/acrylic blends, typically $62-92 \%$ by QUV, $85-97 \%$ in Portland, and $76-104 \%$ in Florida. Gloss retention for Kynar resins on steel substrates were somewhat lower $31-102 \%$ by OUV, 50-112\% in Portland and 0-109\% in Florida. Solvent-based B44 and Incralac coated bronze provided surprisingly low gloss retention during QUV weathering (2-16\% was typical, with B44+BTA providing $81.8 \%$ ), but values were typically higher during outdoor weathering (64-94\% in Portland and typically 45$97 \%$ in Florida). Gloss retention for solvent-based coatings on steel were $13-45 \%$ by QUV, $59-93 \%$ in Portland, and 0-96\% in Florida. The lowest gloss retention for bronze was observed for WL81 coated samples (1-31\% by QUV, $62-83 \%$ in Portland, $71-96 \%$ in 
Florida) with wide variability for steel, likely due to significant film loss during weathering.
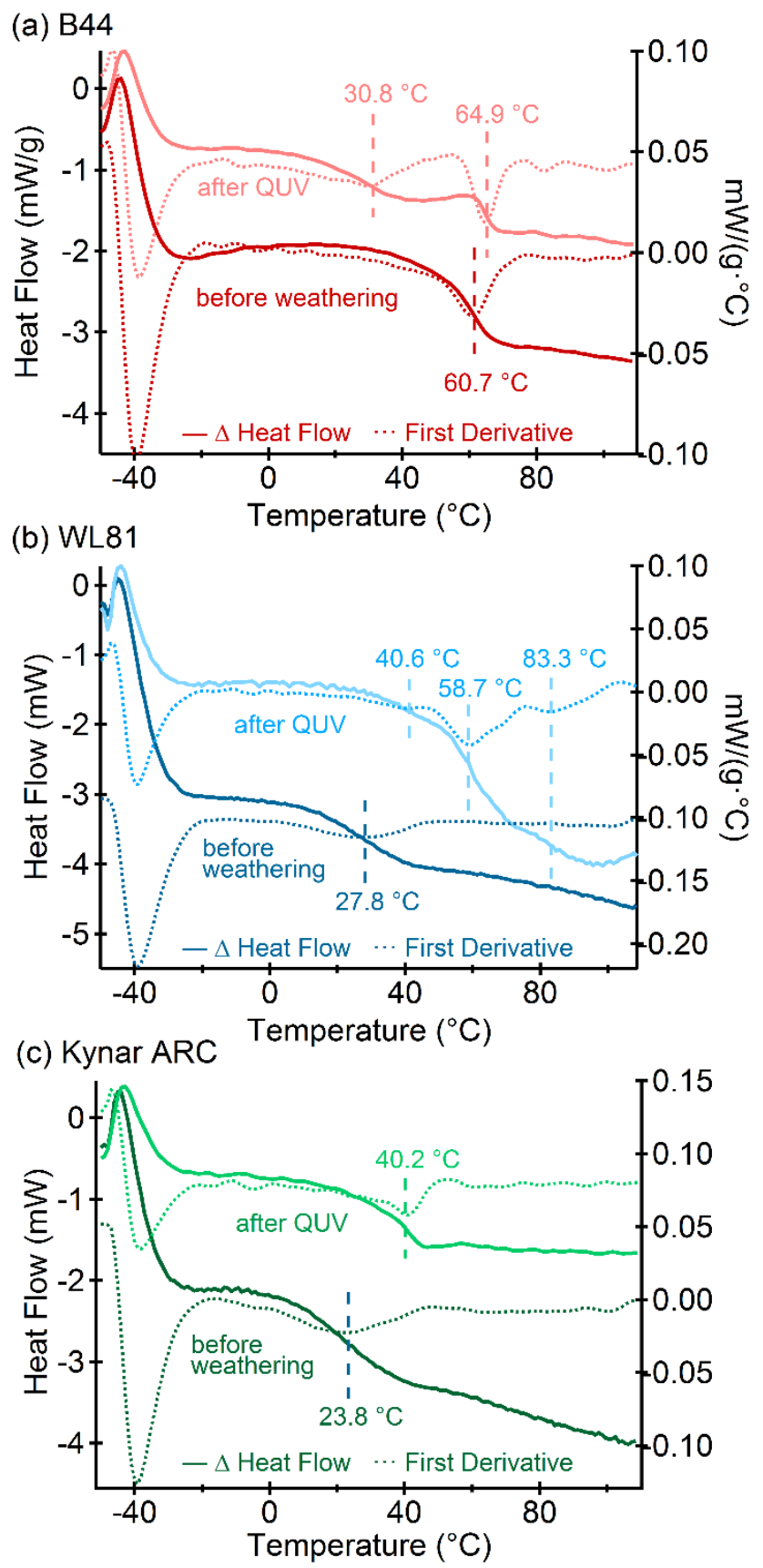

Figure 5.9 DSC thermograms of (a) B44, (b) WL81, and (c) Kynar ARC before and after OUV-B weathering. Change in heat flow is shown with solid lines (left axis) where the first derivative is shown with dashed lines (right axis) and the minimum was used to confirm the respective Tg or MFFT values. 


\subsection{Conclusions}

Latex coatings comprised of a PVDF/Acrylic top coat and compatible B44 primer gave promising results as a substitute for high-performance solvent-based acrylic resins such as B44 alone or Incralac. As evidenced by the EIS data, the B44 and WL81 films without modification were not sufficient for long-term protection of bronze during accelerated or outdoor weathering. The waterborne acrylic resin WL81 showed good workability but higher gloss than wanted and an inadequate protective lifetime. In fact, WL81 was the only coating system tested in this study that performed worse than lifetimes the authors previously reported for waxes subjected to the same weathering conditions. Solvent-based B44 with BTA added showed an increase in lifetime over B44 alone with results comparable to Incralac ( 2000 hours by QUV, 36+ months in Portland, OR, and $18+$ months in S. Florida) but gloss retention was low and some pitting corrosion was visible on the substrate. The addition of a PVDF acrylic blended latex coating as a topcoat (ARC and FMA) improved the performance of the B44 film alone, surpassing any of the solvent-based values and maintaining good barrier properties and excellent gloss retention for the entirety of the study presented here.

Acrylic-containing coatings exhibited a decrease in principal polymer FT-IR peaks, indicating the degradation of acrylic during weathering. FT-IR data suggests that degradation processes included oxidation, side group and chain scission. Evidence of cross-linking was most strongly indicated by thermal analysis of QUV weathered resins with DSC showed chain scission and cross-linking of both B44 and WL81. DSC also showed an increase in MFFT for Kynar ARC, most likely attributed to some cross-linking 
of the acrylic portion in the blend in a similar manner to that of B44. Relative decrease of the acrylic peaks compared to the PVDF peaks in the infrared spectrum provides evidence that degradation of the polymer is occurring in the acrylic component of the resin and not in the PVDF portion, confirming that PVDF is more stable under outdoor exposure than acrylic. Any changes observed in the PVDF coating, either mechanical or chemical can be explained by the breakdown of the acrylic component of the resin. However this process is not only impeded by the inert nature of the substituted fluorines in the PVDF backbone; but also by surface enrichment of PVDF which becomes more crystalline with increased UV exposure. An increase in crystalline domains slows down mass-transport in and out of the film, creating excellent barrier properties even with substantial film thickness loss and retaining larger degradation products in the film to recombine. Overall, spectroscopic and physical analysis of a waterborne PVDF acrylic blend resin indicated its barrier properties equaled or exceeded the solvent-based industry standard.

When independently evaluated by conservators, the ARC was found to be an acceptable level of gloss while traditional Incralac and B44 was generally evaluated as too glossy. The increased workability, long life time, degradation resistance, reversibility and low gloss of the PVDF/acrylic coatings all meet the TRACE criteria and make the ARC and FMA resins an ideal alternative to the current high performance solvent-based coating systems for copper and steel. 


\section{Reducing corrosion of outdoor metalworks using dispersed chemically- stabilized nano-clays in PVDF latex coatings}

Nano-clays are small enough to appear optically transparent, yet they have large surface to volume and high aspect ratios that can significantly inhibit water diffusion when incorporated into films. Clear coatings are commonly applied onto outdoor metalworks, such as sculptures, to prevent corrosion, while minimally affecting the aesthetics of the work. In recent years, waterborne, rather than solvent-based clear coatings are increasingly used in many applications to reduce the volatile organic components in the formulation, yet the performance of dry films produced from waterborne colloidal suspensions is generally poorer. In this work, we aim to improve the barrier properties of a highly weatherable waterborne acrylic/polyvinylidene fluoride (PVDF) emulsion by adding a synthetic nano-clay, Laponite, into the formulation. Strategies investigated for improving clay-polymer compatibility include covalent modification of the edges of Laponite with an acetoxy or perfluoroalkyl silane monomer. Cation exchange on the clay faces using phosphorycholine was conducted and their stability in water was characterized by Zeta potentials. Covalent and ionic modification of nano-clay was verified by XPS and Raman microspectroscopy, changes in interlayer spacing and degree of exfoliation of the clay were monitored by WAXD and SAXS, and barrier properties of the polymer nanocomposite films were characterized by gravimetry, colorimetry and EIS. Surface ablation after accelerated QUV weathering was monitored by ATR-FTIR, TF-XRD, gloss and thickness measurements. Composite films showed many improved properties: reduced water sensitivity and UV degradation and improved barrier properties and diffusion 
constants over both short and long term weathering studies compared to films without nano-clays. The diffusion constant measured for the highest performing composite film showed that the performance gap between relevant water- and solvent-borne coatings used to protect outdoor metals was narrowed by half.

\subsection{Introduction}

With protective coatings, it is possible to reduce corrosion of outdoor metalworks, such as bridges, architectural elements and sculptures, by preventing or slowing the passage of water and ions to the metal substrate. Protective coatings may be pigmented; and pigment particles can further slow mass transport of corrosives through films. However, the use of optically transparent coatings is preferable when the ability to see the substrate is a critical feature for aesthetic and preventative maintenance reasons. Previous work by our group and by others has shown that polymeric coatings containing polyvinylidene fluoride (PVDF) have longer working lifetimes than polyacrylics and other polymers due to excellent chemical and thermal stability. ${ }^{11,48,166-}$

${ }^{167}$ Yet, it has also been shown that water uptake into films produced from waterborne latex coatings is greater than films produced from solvent-based coatings, due to voids in the film caused by defects in spherical close-packing, resulting in a measureable decrease in the films' barrier properties and visible water-whitening of the transparent film. ${ }^{89}$ In this work, we aim to reduce water uptake into those films by incorporating optically transparent nano-clays in aqueous solutions that have been chemicallymodified with acetoxy or perfluoroalkyl-silanes to lower their surface energies and 
increase their dispersability and compatibility within the coating. Incorporation of nano-clays into the resulting dry film should create a tortuous path that would slow diffusion of ions through the coating to the metal substrate. Such composite films would be expected to have increased overall barrier properties, resulting in prolonged working lifetimes and reduced corrosion of the underlying metal substrate. To our knowledge fluorinated nano-clays have not been incorporated into waterborne PVDF/acrylic coatings. The majority of previous studies involving Laponite polymer nanocomposites (PNCs) utilized styrene and butyl acrylate latex ${ }_{,}^{168-169}$ which are not as suitable as PVDF for long-term weatherability.

By incorporating nano-sized inorganics into thermoplastic coatings it may be possible to produce films that have increased hydrophobicity, film hardness and inhibited water diffusion, while retaining optical clarity and easy spray-application. Laponite is a synthetic silicate nano-clay in the smectite family, depicted in Figure 6.1A. The unique properties of clays come from their high aspect ratios (25), specific surface area $(350 \mathrm{~m} / \mathrm{g})$, discoid single crystals and charged surfaces. They are often used as rheology modifiers because the charged interfaces act to control diffusion in wet suspensions and as such have been used to create a range of phases from sols to gels and charged glasses. ${ }^{61}$ Laponite, as well as other smectite clays, have a concentration dependent phase diagram where the amount, $\mathrm{pH}$ and dielectric constant of the bulk solvent play an integral role in inhibiting or facilitating particle interactions. These factors also affect the electrostatic interactions when the clay is added to a colloidal organic polymer suspension. The density and organization of the charges around the 
particle and thickness of the slipping plane can be measured by the Zeta potential, where high potentials (large in absolute magnitude) lead to particle repulsion and low potentials (small in absolute magnitude) lead to flocculation due to small slipping planes that are overcome by attractive forces. At near-neutral $\mathrm{pH}^{\prime}$, expansion of sodium cations out of the interlayer space induces a negatively charged face and partial positive charge along the edge of the platelet as depicted in Figure $6.1 \mathrm{~B} \cdot{ }^{170}$ The mobile interlayer sodium cations encourage the clay to swell in water producing exfoliated (individual) 3-layered (T:O:T) inorganic sheets with the morphology of a platelet. Like many clays, Laponite is highly modifiable in that it has both accessible hydroxyl groups available for silanation on the sheet's broken edge, and exchangeable sodium cations connecting the faces particle-to-particle. ${ }^{171-174}$ In this work, modification of the nanoclay was accomplished by: (1) covalent reactions with acetoxy or perfluoroalkyl silanes

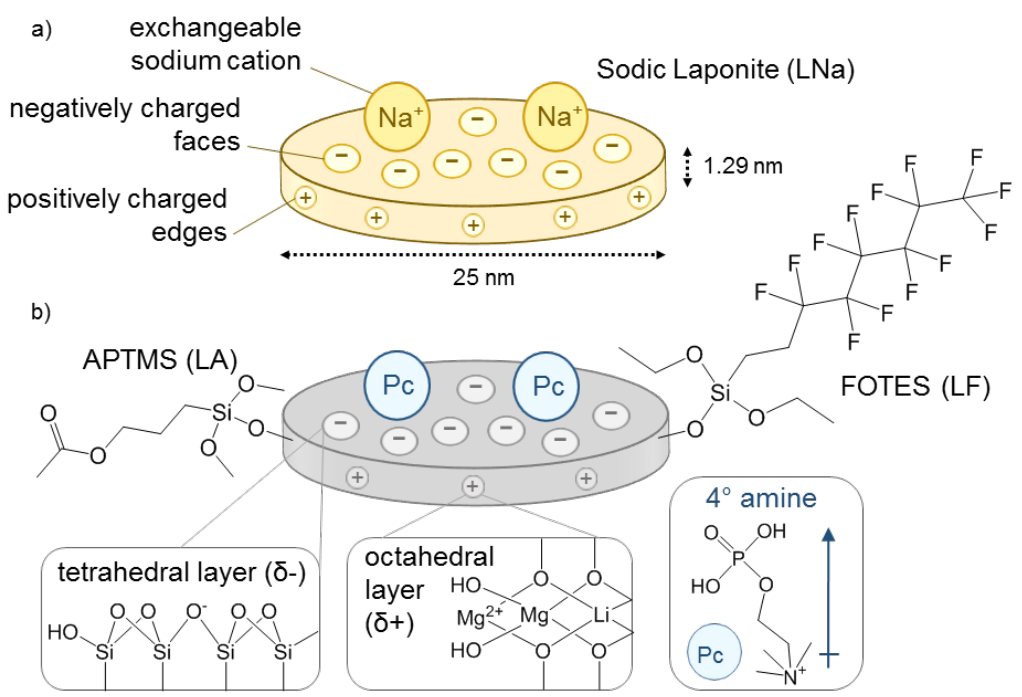

Figure 6.1 The single unit cell formula for Laponite is $\mathrm{Na}_{0.7}\left[\left(\mathrm{Si}_{8} \mathrm{Mg}_{5.5} \mathrm{Li}_{0.3}\right) \mathrm{O}_{20}(\mathrm{OH})_{4}\right]$. A typical $25 \times 1.29$ $\mathrm{nm}$ platelet, abbreviated as $\mathrm{LNa}$ (a), which contains approximately 1000 unit cells per discoid. Chemical modification of clay surface (b) results in covalent attachment of APTMS (LA) or FOTES (LF) at the tetrahedral edge and ionic exchange on the face with phosphoryl choline (PC, resulting in LAPc or LFPc). 
to increase the chemical compatibility between clay and latex particles and (2) cation exchange reactions using phosphoryl choline to charge-stabilize the covalently functionalized nano-clays and promote their exfoliation in water.

The relative strength of stacking (attractive) versus dispersive (repulsive) forces must be carefully balanced to prevent nano-clay agglomeration. With surface modification, the balance of those forces is altered, so the amount and type of surface coverage can have a significant influence on the dispersability. X-ray Photoelectron Spectroscopy (XPS) and Raman spectroscopy were used to verify surface functionalization. Gallery spacing measurements, both before and after modification, were conducted using Wide Angle X-ray Diffraction (WAXD). Particle-particle interactions of wet suspensions of the nano-clays and polymer were characterized by Small Angle X-ray Scattering (SAXS) and Zeta potential measurements.

Mechanical strain due to increased hardness in composite films may result in film cracking upon drying and/or weathering; and latex packing defects may increase the water volume fraction of the material..$^{175-176}$ Other voids and defects may exist or be produced over time from the loss or surface-migration of inorganic fillers (such as pigments or clays). While there are both environmental and cost motivations to produce films from waterborne colloidal polymeric dispersions, the resulting films are more permeable to water due to their chemistry and their film formation mechanism. ${ }^{37}$ Adsorption of water droplets in voids that are sufficiently large to scatter white light, will whiten transparent films. ${ }^{89}$ In this work, mass transport was monitored using colorimetry, gravimetry and spectroscopy. 
To simulate the effects of weathering, the films were exposed to ultraviolet light and condensation cycles in an industry-standard accelerated weathering chamber (QUV). ${ }^{166}$ Electrical impedance spectroscopy (EIS) is a non-destructive technique for monitoring dielectric properties of materials ${ }^{37,} 89,93$ and has been shown to produce similar or more sensitive results as Attenuated Total Internal Reflectance Fourier Transform Infrared Spectroscopy (ATR-FTIR) or gravimetric methods for detecting water-uptake into polymers. ${ }^{177-180}$ Films that enable facile passage of water and electrolytes (i.e. corrosives), are more poorly protective and such films will have larger capacitances and larger diffusion constants than those that are highly protective. By fitting the EIS spectral output to Equivalent Electrical Circuits (EECs), the data can be dissected into frequency ranges in which different circuit elements are dominant. In addition, the values of each element can be monitored for changes over time. Because temperature and humidity fluctuations during weathering may eventually lead to coating failure such as cracking, time-course EIS spectra of films during weathering can be useful in providing insights to the electrochemical changes that occur before the point of coating failure. Gloss and film thickness measurements provided insights into bulk film changes.

During the lifetime of an outdoor coating, significant chemical changes may also occur that have a deleterious effect on its protective quality. The acrylic component of the PVDF resin is chemically similar to the polymer used in the primer coat of the PNCs (PMMA-co-PEA). The acrylic polymer is a commonly used solvent-based coating for the protection of bronzes (Paraloid B-44)..$^{20,93-94, ~ 148, ~ 181 ~ I n ~ p r e v i o u s ~ w o r k ~ w e ~ h a v e ~ o b s e r v e d ~}$ 
that loss of the ester functionality is the primary means of material loss and pathway of degradation for PMMA-co-PEA. ${ }^{54}$ While solvent-based acrylic coatings are commonly used as protective coatings, their susceptibility to chemical degradation during weathering is a notable reason to investigate more highly weatherable substitutes that offer excellent corrosion protection.

\subsection{Experimental}

\subsubsection{Applied coatings}

Preparation of bronze $(2.54 \times 7.62 \mathrm{~cm} ; 90 \% \mathrm{Cu} 10 \% \mathrm{Sn})$ and vinyl (3x8 cm; polyvinyl chloride-co-acetate) substrates are detailed elsewhere. ${ }^{89}$ Basecoat primers were resin dissolved in solvent [Paraloid ${ }^{\mathrm{TM}}$ B-44 resin, principally composed of poly(methyl methacrylate-co-ethyl acrylate), in $20 \%$ (w\%/w toluene), Dow, Inc.] The topcoat used was waterborne Kynar Aquatec ${ }^{\circledR}$ FMA-12 latex [50:50, poly(vinylidene fluoride) and poly(methyl methacrylate) blend, Arkema, Inc.] Substrates (bronze and vinyl) were spray-coated with a Fuji HVLP Super 4 XPCTM for a dry film thickness of approximately 10-15 $\mu \mathrm{m}$ for each layer (i.e. primer base coats or PVDF topcoat) for a total dry film thickness of approximately zo $\mu \mathrm{m}$. Coatings were annealed in an oven for six hours at $60^{\circ} \mathrm{C}$. Accelerated weathering was performed according to ASTM G154 Cycle B: 4 hours UV-B exposure $\left(60^{\circ} \mathrm{C}, \mathrm{E}_{\mathrm{e}}=0.71\right)$ and 4 hours condensation $\left(50^{\circ} \mathrm{C}\right.$, recirculating distilled water). For whitening and mass uptake values films on vinyl were soaked in distilled water and analyzed at four and 72 hours, with three trials of each coating type. Coating masses were taken as the average of three measurements $(\mathrm{N}=3, \mathrm{M}=3)$ and for grayscale 
measurements the films were illuminated for imaging according to ASTM D1729-96. Images were recorded with a Nikon $\mathrm{D}_{40} \mathrm{O}$ and converted to grayscale in Adobe Photoshop CC, where the average $k$ value $(\mathrm{N}=3, \mathrm{M}=9)$ of the chart was obtained. Gloss and thickness measurements were acquired as an average of five trials with a Gardco $\mu$ Tri-Gloss meter.

\subsubsection{Preparation of clays}

Laponite RD and S482 (Southern Clay Products, Inc.) and silylating agents 3acetoxypropyltrimethoxysilane (APTMS, Gelest, Inc.) and (tridecafluoro-1,1,2,2tetrahydrooctyl) triethoxysilane (FOTES, Gelest, Inc.) were used as supplied. To obtain covalently modified clays, toluene was distilled directly into a flask containing Laponite $\mathrm{RD}$ (1 g nano-clay/100 $\mathrm{mL}$ solvent) under anhydrous conditions in a closed system $\left(\delta^{+} \mathrm{N}_{2}\right.$ flow). After 30 minutes of heating with stirring at $35^{\circ} \mathrm{C}$, the silane was added to the toluene clay mixture ( $1 \mathrm{mmol} / \mathrm{g}$ clay) and the contents in the flask were left to stir an additional four hours at the same temperature. The covalently modified Laponite was isolated for diffraction analysis by vacuum filtration with a $0.2 \mu \mathrm{m}$ Nylon membrane, washed extensively with toluene then dioxane and dried $12 \mathrm{~h}$ at $65^{\circ} \mathrm{C}$. The aqueous dispersions of modified clay (LA: Laponite-APTMS or LF: Laponite-FOTES) were obtained by quenching the grafting reaction with $50 \mathrm{~mL}$ of water and isolating the water-stable nano-clay by liquid-liquid extraction in a separatory funnel. For cation exchange aqueous dispersions of LA and LF were diluted to $100 \mathrm{~mL}$ and heated to $50^{\circ} \mathrm{C}$ after which one equivalent ( $C E C_{\text {Laponite }}=0.75 \mathrm{mmol} / \mathrm{g}$ clay) of phosphocholine chloride 
calcium salt tetrahydrate (PC) was added and stirred for 12 hours. The exchanged/grafted clay (LAPC and LFPC) was vacuum filtered, washed with cold ethanol and immediately re-dispersed in DI water. Aqueous dispersions $(2.6 \% \mathrm{w} / \mathrm{w})$ of clays of LAPC, LFPc or Laponite S482 (LNa: unmodified sodic Laponite blended with peptizing agent tetrasodium pyrophosphate) were added with high shear to freshly prepared PVDF latex immediately after preparation and no aging/gelation of the clay was observed at time of incorporation. The presence of chemical modifiers at each step was initially confirmed by ATR-FTIR. ${ }^{54}$

\subsubsection{Characterization}

A working electrode area of $14.6 \mathrm{~cm}^{2}$ was saturated with electrolyte $(3 \% \mathrm{NaCl})$ for EIS analysis with a Gamry Reference 6oo Potentiostat from $1 \mathrm{MHz}-0.1 \mathrm{~Hz}$ with AC voltage $20 \mathrm{mV}$ rms and $\mathrm{DC}$ voltage $0.0 \mathrm{~V}$ vs. open circuit potential $(0 \pm 200 \mathrm{mV})$. Spectra were interpreted and modeled against equivalent electrical circuits (EECs) with Scribner Associates ZView. Errors in fit EEC models to the data were calculated as the sum of residual error where the fit differed from the experimental data. Capacitance values for diffusion analysis were calculated at $10 \mathrm{kHz}$ according to $C=-1 /\left(w \cdot Z_{i}\right)$, where $w$ is the radial frequency and $\mathrm{Zi}$ is the imaginary component of impedance. Zeta-potentials were measured on filtered aqueous clay dispersions with a Malvern Zetasizer Nano and disposable plastic cells. Thermogravimetric measurements were acquired in platinum crucibles at a scanning rate of $20^{\circ} \mathrm{C} / \mathrm{min}$ from $50-750^{\circ} \mathrm{C}$ using a Perkin-Elmer TGA7. 
ATR-FTIR spectra were acquired with a Nicolet Continu with a ThermoScientific iS 10 infrared spectrometer and a $50 \mu \mathrm{m} \mathrm{MCT} \mathrm{detector}\left(4 \mathrm{~cm}^{-1}\right.$ resolution) operated with Omnic. Spectra were acquired using a diamond ATR objective to enable in-situ analysis of a roughly circular area with a $250 \mu \mathrm{m}$ diameter. Data was transformed using an N-B strong apodization function and Mertz phase correction. Raman spectra were measured with a Horiba LabRam microscope using a $532 \mathrm{~nm}$ diode laser ( $22 \mathrm{~mW}), 50 x$ objective (200 $\mu \mathrm{m}$ confocal hole), $1800 \mathrm{gr} / \mathrm{mm}$ grating and LabSpec 6 software. All X-ray studies were acquired with Rigaku Ultima IV Multipurpose X-ray diffractometer with a Cu-k $\alpha$ radiation source $(\lambda=1.542 \AA)$ and step size $=0.002 \mathrm{deg}$ for $12 \mathrm{sec}$. Powder samples were ground finely with an agate motor and pestle and pressed into a random orientation on a mirrored slide. For scattering studies in transmission mode, thin-walled glass capillary tubes $(1.5 \mathrm{~mm}$, CharlesSupper, Co.) were filled with either the aqueous clay dispersions or wet resin and attached to a $20 \mathrm{~mm}$ sample window fixed in place near the vacuum path window. Thin film spectra with or without a $0.5^{\circ}$ grazing angle were acquired in-situ after alignment of the sample in the $x_{-}, y_{-}$, and $z$-directions. XPS measurements were performed on vacuum dried clays with a Phi VersaProbe II using an Al ka anode (10 $\mu \mathrm{m}$ spot size) and MultiPak software.

\subsection{Surface modification of clays}

Covalent modification of Laponite nano-clays with APTMS or FOTES silanes occurs via a reaction between the hydroxyl groups on the edge of the clay sheet and a silane bond 
to produce covalent siloxane bonds, as illustrated by the products shown in Figure 6.1B. To promote the exfoliated state (aqueous isotropic), ionic exchange with phosphorylcholine tetrahydrate (Pc) was undertaken to create covalently-modified and cation-exchanged nano-clays, abbreviated LAPC and LFPc. Surface coverage, successful covalent attachment of the silanes and amount of physisorbed water on the clay particles were investigated by XPS, WAXD, TGA, and Raman; a summary of this data is presented in Appendix $\mathrm{F}$.

Integration of regions $\mathrm{Na}$ 1s, $\mathrm{Si} 2 \mathrm{p}, \mathrm{Mg} 2 \mathrm{~S}, \mathrm{Li}$ 1s, and $\mathrm{O}$ 1s peaks in the XPS scan of LNa confirmed the empirical formula of the clay's unit cell (Figure 6.2). High resolution scans of clay LF showed peaks at 688,683 and $102 \mathrm{eV}$ that correspond to the binding energy of $F 1 s$ and Si $2 p$ orbitals, and were fit with a Lorentzian peak shape for quantitation. The two peaks in the F 15 scan are due to organic C-F bonds in the silane molecule and the metallic Si-F bonds from a small amount of direct fluorination of the silicate, which is likely a byproduct of the sample and X-ray beam interaction. The peak ratio of fluorine from the peak at $688 \mathrm{eV}$ to silicon at $102 \mathrm{eV}$ indicated that on average each $25 \mathrm{~nm}$ disctoid had 690 FOTES molecules on its surface. As discussed in Appendix 6-1, calculations of the data from WAXD measurements determined that each unmodified clay LNa disc had approximately $1.05 \times 10^{3}$ unit cells and 290 accessible docking sites along the rim, which provided a grafting efficiency of $228 \%$ for LF. TGA analysis of LF showed a gravimetric decrease upon heating and quantitatively revealed that $35 \%$ of the FOTES molecules were physisorbed to the clay surface. When the number of FOTES molecules per clay stack was adjusted by $-35 \%$, the result is $148 \%$ 
covalent grafting efficiency of the docking sites or on average 1.5 FOTES molecules/accessible hydroxyl. Although some have reported individual silanes covalently bridging multiple clay sheets, ${ }^{169,}{ }^{171-172,182}$ the geometric constraints of these molecules make that scenario unlikely. Our grafting efficiency of 1.5 suggests that cross-linking reactions between silane molecules occurred, resulting in an average of 12 molecules bound per site.

Having hydrophilic nano-clays is important to prevent flocculation or gelation of the clays upon addition to the aqueous coating dispersion. Since active Raman modes are very sensitive to their environment, characteristic peaks of the layered silicates are shown in Figure 6.3 and include a number of $\mathrm{O}-\mathrm{H}$ stretches from metal-hydroxyl

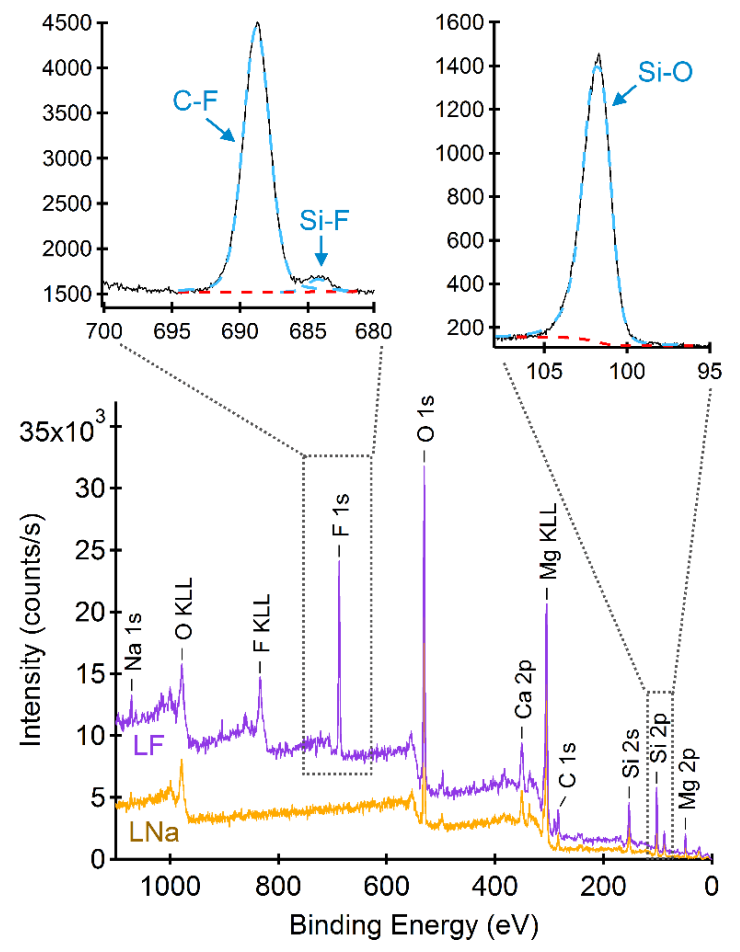

Figure 6.2 XPS full scan of Laponite clay modified with FOTES (LF, red) and expansions of Fluorine 15 and Silicon $2 p$ regions used in quantitation. Peaks used in peak fitting of data are shown with dashed lines overlapping the spectrum. Unmodified clay ( $\mathrm{LNa}$, black) is shown for comparison. The KLL lines are Auger emissions (Auger lines were not used in quantitation.). 
complexes (Si-OH), $3620 \mathrm{~cm}^{-1}$ (inner sheet $\mathrm{M}-\mathrm{OH}$ ), $3425 \mathrm{~cm}^{-1}$ (amorphous $\mathrm{H}-\mathrm{OH}$, bulk water), and $3200 \mathrm{~cm}^{-1}$ (in phase $\mathrm{H}-\mathrm{OH}$ with crystalline structure). As a measure of hydrophilicity, it is possible to compare the amount of physisorbed water on the different modified nano-clays using the intensity ratio of the Raman shift of bulk water (at $\left.3450 \mathrm{~cm}^{-1}\right)$ to the silicon-oxide lattice of the nano-clay $\left(\mathrm{Si}_{-} \mathrm{O}_{4}\right.$ at $683 \mathrm{~cm}^{-1}$, shown in Appendix F, Figure $\mathrm{F}_{1}$ ). ${ }^{183}$ The hydroxyl stretching bands shown in Figure 6.3 (and ratios listed in Appendix F, Table $F_{1}$ ) indicate that the largest hydrophilicity ratio was observed for unmodified clay $\left(\mathrm{I}_{2} \mathrm{O} / \mathrm{Si}-\mathrm{O}_{4}=0.37\right)$. The amount of bulk water in the nanoclay decreased significantly upon modification with the organosilane and subsequently increased after cation exchange $(L A=0.18$ to $L A P C=0.29$ and $L F=0.12$ to $L F P c=0.19)$. Similar trends were observed by TGA where unmodified clay LNa had twice as much total water adsorbed by mass than LA or LF (Appendix F, Figure $F_{3}$ ). The higher water content in the Pc exchanged clays is consistent with having successfully transitioned the covalently-modified nano-clays from organophilic to hydrophilic post-cation exchange. Both the polar tail of Pc as well as the occasional exchange of monovalent sodium for a divalent calcium counter-ion, which increases the hydration sphere for atmospheric water, likely explains the increased water sorption observed for the Pc compared to the non-Pc clays.

Clays are considered to be stable (i.e. unlikely to flocculate) in dilute dispersions that have Zeta potentials, $\zeta$, more negative than $-30 \mathrm{mV} .{ }^{184}$ Table 6.1 lists the measured electrokinetic potentials of each clay after undergoing covalent modification and cation exchange. Unmodified LNa had a $\zeta=-42.9 \mathrm{mV}$ when dispersed in water at neutral $\mathrm{pH}$. 
The grafted/exchanged clays had slightly increased potentials of approximately $-38 \mathrm{mV}$ for both LAPc and LFPc. It is important to note that cation-exchanged clays (LAPC and LFPc) were stable in water, whereas those that had not been exchanged (LA and LF) were not stable in water. From these data, we demonstrate that such modifications to clays can result in aqueous stable dispersions.

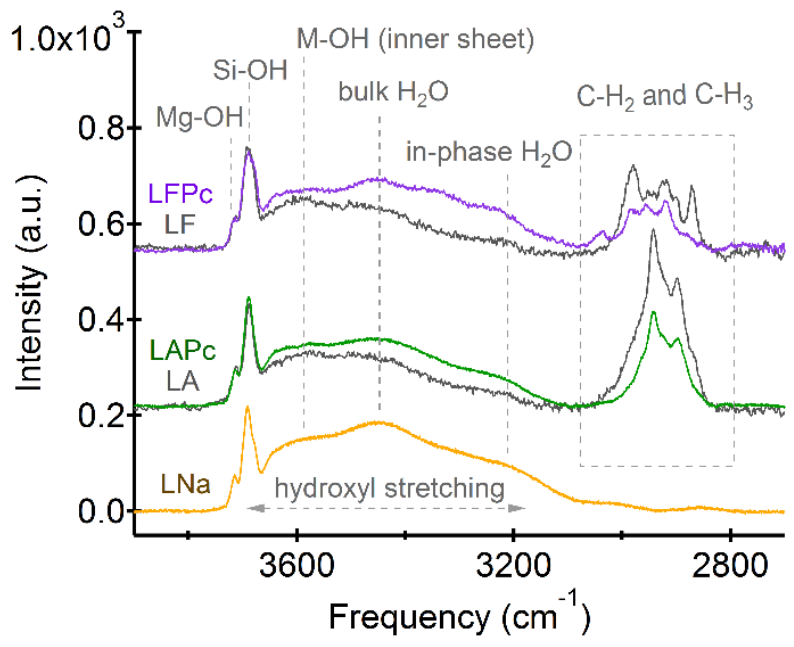

Figure 6.3 Raman spectra of the hydroxyl and aliphatic regions of unmodified and functionalized nanoclays, normalized to the $\mathrm{Si}_{-} \mathrm{O}_{4}$ band at $683 \mathrm{~cm}^{-1}$. There are typically 1.4 water molecules coordinated to each $\mathrm{Na}^{+}$and 4.4 water molecules coordinated to each $\mathrm{Ca}^{2+}$ (the counter ion for Pc used in cation exchange). There are two hydroxyl binding sites in the clay crystal structure, $\mathrm{M}-\mathrm{OH}$ denotes inner sheet hydroxyls attached to a silicon $(\mathrm{Si}-\mathrm{OH})$ or magnesium $(\mathrm{Mg}-\mathrm{OH})$. The vibrations of external hydroxyls are at higher frequencies as labeled.

Table 6.1 Particle size and stability in aqueous and colloidal suspensions

\begin{tabular}{|c|c|c|c|c|c|c|c|}
\hline & $\underset{(m V)}{\zeta}$ & $\begin{array}{l}\mathrm{t}_{\mathrm{H}_{2} \mathrm{O}} \\
(\mathrm{nm})\end{array}$ & $\begin{array}{l}t_{\text {PVDF }} \\
(\mathrm{nm})\end{array}$ & $\begin{array}{l}\mathrm{d}_{\mathrm{PVDF}} \\
(\mathrm{nm})\end{array}$ & $\begin{array}{l}\text { Neighbor } \\
(\mathrm{nm})\end{array}$ & $\begin{array}{l}\text { Volume }_{\text {PVDF }} \\
(\%)\end{array}$ & $\begin{array}{l}\text { Size Dist } \\
(\%) \\
\text { PVDF }\end{array}$ \\
\hline \multirow{2}{*}{ LNa } & \multirow{2}{*}{-42.9} & \multirow{2}{*}{1.13} & C 1.19 & $24 \cdot 3$ & 1.94 & 0.16 & 5.00 \\
\hline & & & P 235.9 & 202.2 & 11.0 & 99.6 & 16.4 \\
\hline \multirow{2}{*}{ LAPC } & \multirow{2}{*}{-37.8} & \multirow{2}{*}{1.68} & C 1.72 & 29.2 & 1.63 & 0.34 & 14.1 \\
\hline & & & P 212.8 & 199.2 & 14.1 & 99.7 & 12.9 \\
\hline \multirow{2}{*}{ LFPC } & \multirow{2}{*}{-38.3} & \multirow{2}{*}{1.51} & C 1.44 & 29.4 & 1.30 & 0.31 & 3.20 \\
\hline & & & P 221.2 & 188.9 & 11.4 & 99.7 & 9.70 \\
\hline
\end{tabular}

$\zeta=$ Zeta potential, $\mathrm{T}=$ thickness, $\mathrm{D}=$ diameter, $\mathrm{C}=$ clay, $\mathrm{P}=$ polymer. $\mathrm{H}_{2} \mathrm{O}$ values were calculated by SAXS using a cylinder model, and PVDF values were calculated by SAXS with spheroid model. 


\subsection{Nano-clays in colloidal suspensions}

In order to understand the structure of the modified nano-clays, SAXS studies were undertaken to ensure adequate exfoliation in both aqueous solutions and in the polymer latex suspension. Based on particle sizes measured, these data showed that small, exfoliated clay platelets were produced (Appendix F, Figure F4); the relevant values from which are included in Table 6.1 for comparison in this discussion. A twodispersion spheroid model was fit to the data to determine particle sizes of latex and clay particles in water as shown in Figure 6.4. The spheroid shape permitted flexibility in dimensions for multiple populations in the dispersion (i.e. the nano-clays with an aspect ratio $\approx 0.05$ and the latex polymer with an aspect ratio $\approx 1$ ). Fit diameters of the dispersed nano-clays (both in water and aqueous PVDF latex) are shown in Table 6.1. Importantly, the diameter of the clay when mixed with polymer $\left(d_{\text {PVDF }}\right)$ is quite similar to the diameter of the clay $\mathrm{d}_{\mathrm{H}_{2} \mathrm{O}}$ in water, indicating that the clay does not aggregate upon addition to the latex suspension. The closeness in scattering values and narrow polydispersity of clay populations obtained validates the use of the two-dispersion spheroid model for these mixed suspensions.

Using the intensity scale of each population in the dispersion, the volume fraction of scattering clay particles was, on average, $0.26 \%$, which is in excellent agreement with the theoretical volume fraction of $0.28 \%$, that was calculated using the density of Laponite $\left(2.53 \mathrm{~g} / \mathrm{cm}^{3}\right)$, density of PVDF resin $\left(1.19 \mathrm{~g} / \mathrm{cm}^{3}\right)$ and the $0.6 \%$ loading mass of clay. The small size and broad shape of the diffraction peak at $0.3-0.4$ $\mathrm{A}^{-1}$ suggested that the dispersion was highly disordered (poorly crystalline), which 

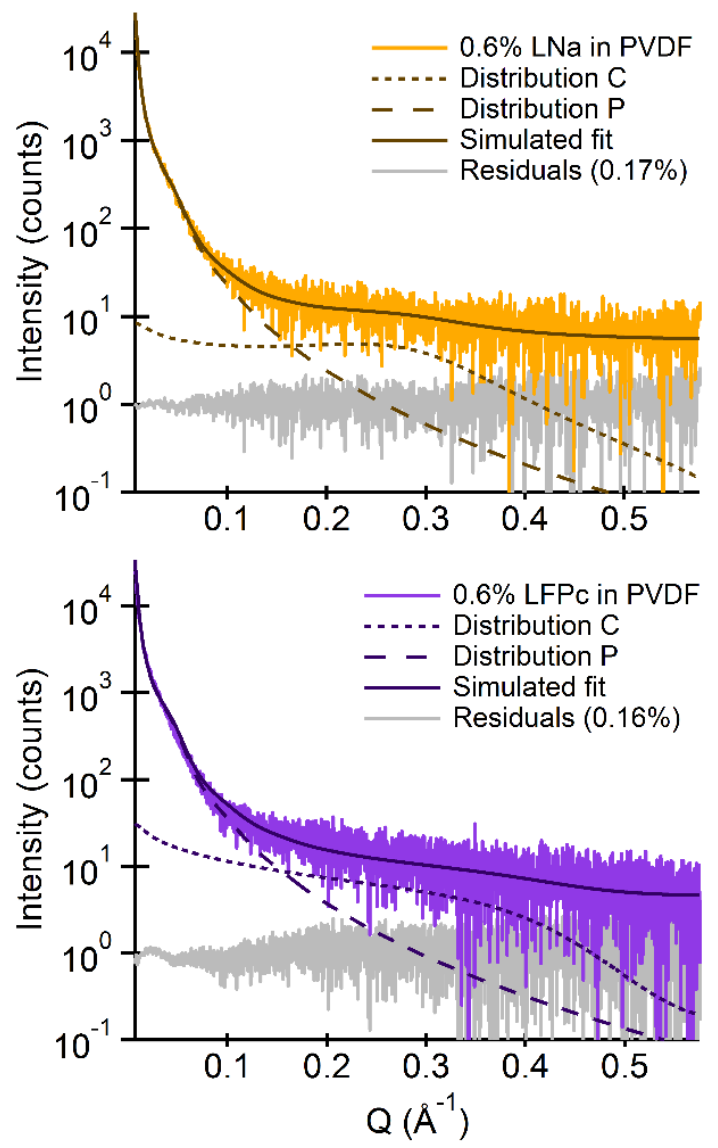

Figure 6.4 SAXS models of modified nano-clays (C) in PVDF latex dispersion (P). Data shown is for LNa (top) and LFPc (bottom).

would be expected from randomly dispersed particles. The close particle-neighbor interactions and the flat profile of the clay scattering patterns suggest that any ordering is nematic ${ }^{185}$ and any stacking that occurs is on a short scale in both distance and time.

\subsection{Barrier properties of waterborne PVDF-clay nano-composites}

\subsubsection{Mass-transport}

The grayscale values of fresh and annealed PNC films measured after water-soaking for 4 and 72 hours are shown in Figure 6.5, where black= $0 \%$ and the film was completely transparent while white $=100 \%$ and the film was completely opaque. (Coatings are often annealed to aid in driving off volatile hydrophilic additives, which happens 
naturally with weathering, though over a prolonged time period.) While the grayscale values were similar across films after soaking for four hours, sustained soaking to 72 hours showed water whitening was reduced by $50-65 \%$ when chemically modified nano-clays were added compared to PVDF films without the clays. These data suggest that the numbers and/or volumes of light scattering voids present in the final films were reduced by adding nano-clays LAPc and LFPc into the wet suspensions, but not LNa.

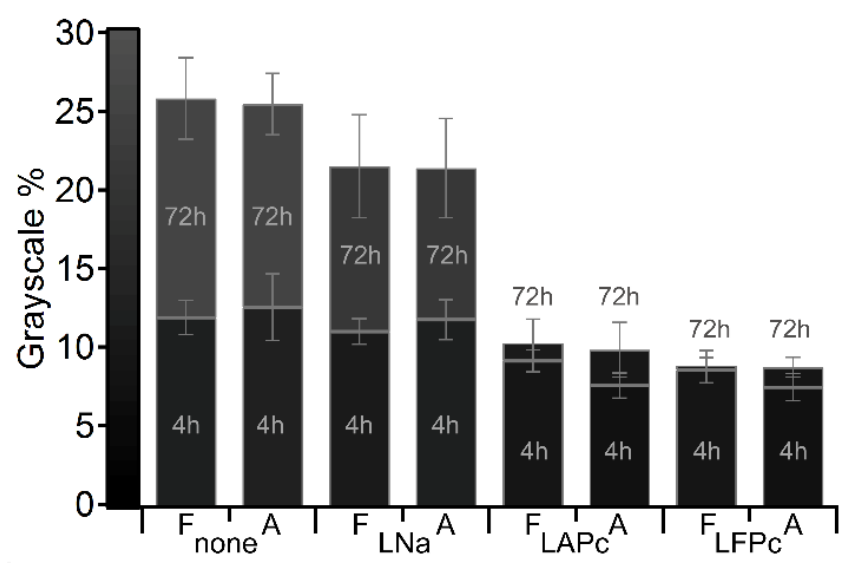

Figure 6.5 Whitening of the fresh (F) or annealed (A) films on black reference substrates after soaking in electrolyte for four and 72 hours. The initial grayscale values of all films was approximately $8 \%$.

Permeability tests of the fresh (dry, but not weathered) films were conducted by allowing each coated substrate to soak in electrolyte until saturation and the capacitance was monitored as an impedance measurement at $10 \mathrm{kHz}$, using the imaginary part of the impedance as the capacitance. Relative capacitance $\left(C / C_{0}\right)$ of annealed films versus $\sqrt{ } \mathrm{t}$ was plotted in Figure 6.6a. The traces showed that diffusion through the films occurred in two stages: initial linear uptake and then a slow percolation until the films became saturated. The initial uptake profiles $\left(35-200 \mathrm{~s}^{1 / 2}\right)$ in Figure 6.6a of annealed PVDF and PVDF+LNa are considered to be type II Fickian diffusion, meaning that the pores are large enough that they do not restrict movement 
of ions. In contrast, the decreased slopes of the films with LFPc and LAPc are indicative of a material having a more constrained diffusion profile than for the other two films. The diffusivity of each coating to water can be quantified by the time it takes to saturate a film and the magnitude of the films' capacitance..$^{177^{-179}}$ The diffusion coefficients of water $\left(\mathrm{D}_{\mathrm{H}_{2} \mathrm{O}}\right)$ through the fresh and annealed films are listed in Table 6.2. The fresh PVDF coating without nano-clays has the freest ion transport, $2.4 \times 10^{-9} \mathrm{~cm}^{2} / \mathrm{s}$. Coatings with covalently modified nano-clays, LAPc and LFPc, had diffusion rates that averaged $4.5-1.5 \times$ slower than for the LNa and PVDF only films and took a significantly longer time to reach saturation. The uptake profiles of LAPC and LFPc in Figure 6.6a show constrained and nearly single-file diffusion. Calculations have demonstrated that ion movement is restricted when pores are narrowed to less than twice the diameter of the diffusing species. ${ }^{68,186}$ For water, having a diameter of $\sim 0.3 \mathrm{~nm}$, the size when movement becomes restricted is $0.6 \mathrm{~nm}$.

(a)

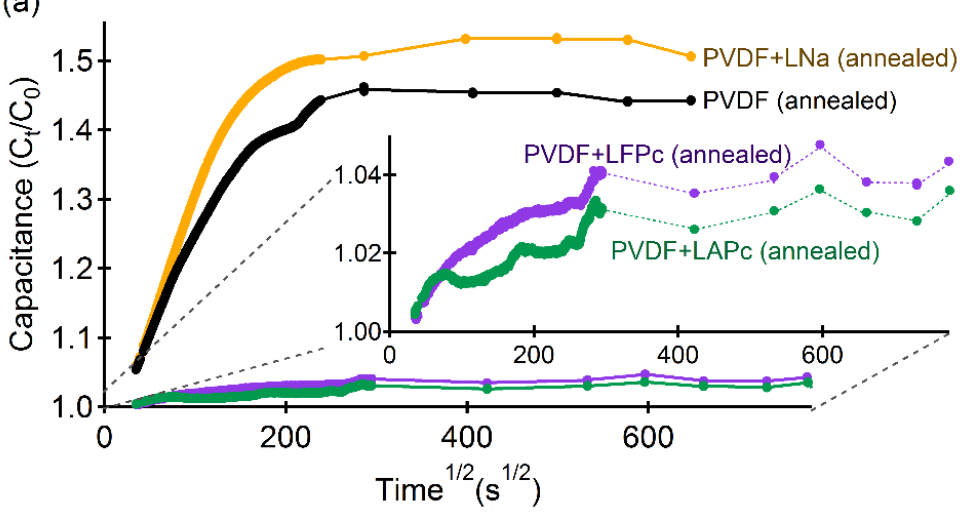

(b)

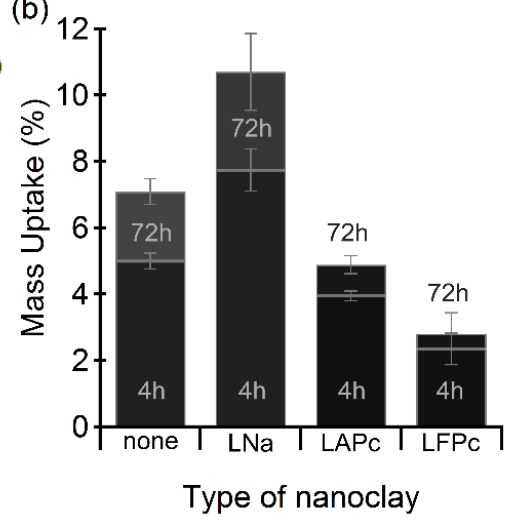

Figure 6.6 Diffusion profiles of electrolyte using relative capacitance of the films over time is shown in (a), with the inset provided for visual clarity for two of the films. In (b) the mass uptake percentage for various annealed coatings after soaking in water for $4 \mathrm{~h}$ and $72 \mathrm{~h}$ is shown. The shade of each bar represents the grayscale value Figure 6.5. 
Given that these latex particles have an average particle size of $197 \mathrm{~nm}$, as determined by SAXS and assuming spherical close-packed models, the maximum calculated diameter of the interstitial spaces from octahedral voids is $82 \mathrm{~nm}$ and from tetrahedral voids it is $44 \mathrm{~nm}$. Those interstitial void dimensions are smaller than the wavelength of visible light and thus not likely to cause scattering/whitening, rather packing defects that result in larger pores must be present to observe whitening. However, given the relatively free diffusion observed in the LNa composite film compared to the film without clay, it seems likely that the high surface energy of the clay resulted in aggregation in voids and at the surface, ${ }^{187}$ decreasing the film whitening (as voids would be clay-filled) and larger mass uptake (as the unmodified clays are highly charged).

Table 6.2 Barrier properties of the films before and after 4250 hours QUV-B

\begin{tabular}{|c|c|c|c|c|c|}
\hline & $\begin{array}{l}\mathrm{D}_{\mathrm{H}_{2} \mathrm{O}} \\
\left(\mathrm{cm}^{2} / \mathrm{s}\right) \\
\text { fresh }\end{array}$ & $\begin{array}{c}\mathrm{D}_{\mathrm{H}_{2} \mathrm{O}} \\
\left(\mathrm{cm}^{2} / \mathrm{s}\right) \\
\text { annealed }\end{array}$ & $\begin{array}{c}|\mathrm{Z}|_{\mathrm{o.1Hz}} \\
\left(\mathrm{M} \Omega \cdot \mathrm{cm}^{2}\right) \\
\text { Initial }\end{array}$ & $\begin{array}{c}|\mathrm{Z}|_{\mathrm{o} \cdot \mathrm{Hz}} \\
\left(\mathrm{M} \Omega \cdot \mathrm{cm}^{2}\right) \\
\mathrm{OUV} 4 \cdot 5 \mathrm{kh}\end{array}$ & $\begin{array}{l}\text { Electrochemica } \\
\text { I Stress Index }\end{array}$ \\
\hline PVDF & $2.4 \times 10^{-9}$ & $4.8 \times 10^{-10}$ & 536 & 34.8 & 2.66 \\
\hline $\mathrm{PVDF}+\mathrm{LNa}$ & $5.0 \times 10^{-9}$ & $5.3 \times 10^{-10}$ & 2,910 & 417 & 1.48 \\
\hline$P V D F+L A P C$ & $8.6 \times 10^{-10}$ & $3.7 \times 10^{-10}$ & 3,490 & 1,530 & 0.993 \\
\hline$P V D F+L F P C$ & $7.6 \times 10^{-10}$ & $2.6 \times 10^{-10}$ & 4,210 & 2,390 & 0.987 \\
\hline
\end{tabular}

For comparison, diffusion constants $\left(D_{\mathrm{H}_{2} \mathrm{O}}\right)$ for annealed coatings: Wax $=1.31 \times 10^{-9}$ $\mathrm{cm}^{2} / \mathrm{s}$, SB Acrylic $=1.46 \times 10^{-10} \mathrm{~cm}^{2} / \mathrm{s}$, WB PVDF $70: 30=3.95 \times 10^{-10} \mathrm{~cm}^{2} / \mathrm{s}$

Having gained an understanding of the presence of light scattering voids and permeability profiles of the composite films, the entire water content within soaked films was measured by mass uptake studies. The data shown in Figure $6.6 \mathrm{~b}$ reveals that there is a much larger mass increase for the annealed films of PVDF and PVDF + LNa than for those with modified nano-clays after soaking for both 4 and 72 hours. Aside from having higher surface energy and clay-filled voids, that the LNa films absorbed 
significantly more water than the other nano-composite films may be due to a nonreversible expansion of the mineral structure of LNa upon heating (annealing) or soaking ${ }^{188}$ which is a response that is typically reduced post-cation-exchange (i.e. as in LAPc and LFPc). It is also possible that unmodified nano-clays are more easily washed out during soaking, resulting in production of voids formerly occupied by the clays, which may have occurred for the LNa clays more than for the modified clays. It is likely that films containing modified nano-clays showed improved barrier properties for two reasons. Firstly, the nano-clay itself provided a tortuous pathway for water. Secondly, the covalently-linked molecules have similar chemistry as the polymer (with APTMS being similar to the acrylic portion and FOTES being similar to the PVDF portion), reducing the surface energy difference between polymer and clay and encouraging the formation of an interpenetrating network in the composite, rather than clay aggregation in the interstitial voids. From the very small increase in whiteness value seen after $72 \mathrm{~h}$ compared to $4 \mathrm{~h}$ soaking times of the modified composite films, the data also suggest that annealing post-application is not necessary for those films. While the data in Figs. 5 and 6 showed significantly improved performance characteristics in modified composite films, those data showed little differentiation between the effectiveness of the acrylic versus the perfluoroalkyl modified clays in corrosion prevention. Furthermore, the long-term performance profiles of the composite films were unknown. A study using accelerated UV weathering of the films with characterization by EIS was undertaken to gain greater insight into the transport of electrolytes through the films over time. The aggressive weathering conditions were 
useful in determining if either chemical-modification scheme provided a significant long-term film performance improvement.

\subsubsection{Impedance and circuit values as metrics of protective quality}

Total impedance at low frequencies $\left(|\mathrm{Z}|_{0.1 \mathrm{~Hz}}\right)$ is usually dominated by the coating resistance $\left(R_{\text {coat }}\right)$ or charge transfer $\left(R_{c t}\right)$ resistance when the $|Z|$ is large. Regardless of circuit element attribution, decreases in the value of the impedance at $0.1 \mathrm{~Hz}$ are correlated with decreases in the film's barrier properties, where the minimum acceptable value of protective films $\mathrm{s}^{82}$ is $10 \mathrm{M} \Omega$ (or in our cell setup, $146 \mathrm{M} \Omega \cdot \mathrm{cm}^{2}$ ). Impedance values for these films are listed in Table 6.2 and plotted over 4500 hours of weathering in Figure 6.7. Low-frequency impedance increased for all films upon OUV weathering, likely due to annealing and other film morphological/chemical changes, before it decreased from its maximum value after $1500 \mathrm{~h}$ of exposure. By this metric, the LFPc film was the best performing coating as it retained a high initial impedance of 4,210 M $\Omega \cdot \mathrm{cm}^{2}$ at $0.1 \mathrm{~Hz}$ during weathering with a final value of $2,390 \mathrm{M} \Omega \cdot \mathrm{cm}^{2}$ after weathering (-43\%). The film with the worst barrier properties, PVDF (without nanoclays) had an initial impedance of $536 \mathrm{M} \Omega \cdot \mathrm{cm}^{2}$ and showed the most drastic decrease to 34.8 $\mathrm{M} \Omega \cdot \mathrm{cm}^{2}(-93 \%)$, making it no longer considered to be protective. The film with LNa added had an initial impedance of 2,910 $\mathrm{M} \Omega \cdot \mathrm{cm}^{2}$, which showed improvement over PVDF without nano-clay and retained overall barrier properties during weathering, and a final impedance of $417 \mathrm{M} \Omega \cdot \mathrm{cm}^{2}$, a decrease of $-86 \%$ that approached the minimum protective impedance value for coatings. The coating with LAPC showed 

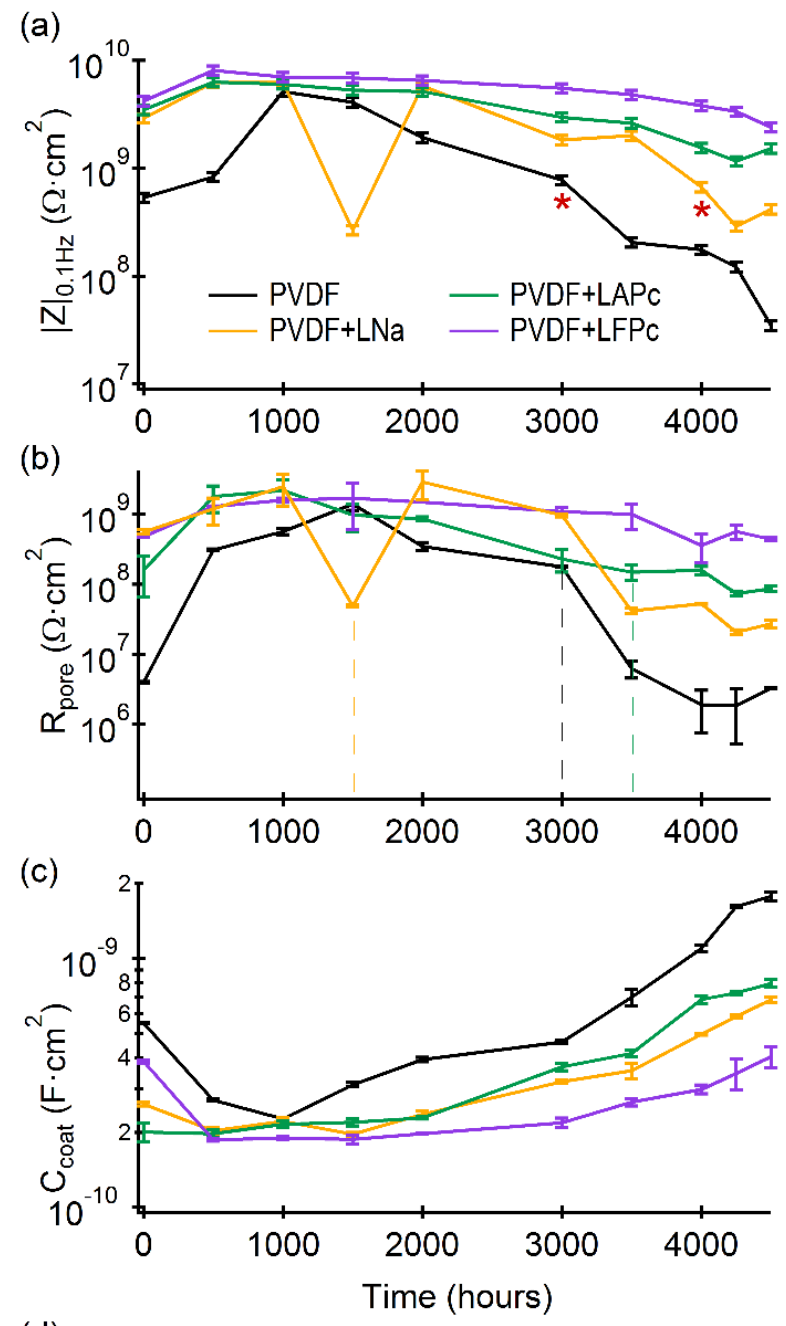

(d)

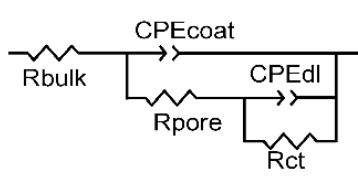

Before Weathering

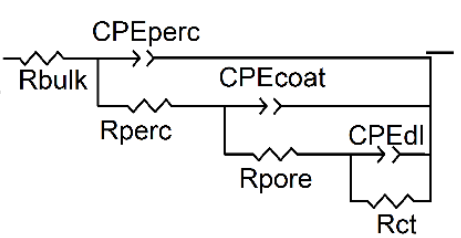

Failure after Weathering*

Figure 6.7 Impedance analysis of the films with and without nano-clays. Plots of a) total impedance at low frequency $0.1 \mathrm{~Hz}$ as measured over 4500 hours of OUV-B weathering, where the asterisk (*) marks the transition to a more complex EEC, when percolation through the top layer of the coating became measurable. Coating resistance (b) and capacitance (c) are plotted using the 'Before Weathering' model until $3000 \mathrm{~h}$ for PVDF and $4000 \mathrm{~h}$ for PVDF+LNa; thereafter the 'Failure after Weathering' model was used. Dashed vertical lines in (b) mark the time point when corrosion on the substrate became visible as small circular brownish dots on the samples.

similar performance to LFPc films by having a high initial impedance of $3,490 \mathrm{M} \Omega \cdot \mathrm{cm}^{2}$ and a relatively small $(-53 \%)$ decrease in barrier properties yielding a final impedance of 
1,530 $\mathrm{M} \Omega \cdot \mathrm{cm}^{2}$. As predicted by the small mass transport values, this EIS data shows that coatings with chemically modified nano-clays retained their protective qualities the most during weathering.

It is also useful to consider changes in key EEC elements compared to their initial values over time. From model-fit values of pore resistance and coating capacitance in Figure $6.7 \mathrm{~b}-\mathrm{c}$, a similar trend to impedance values can be seen and is discussed in more detail in SI Section 4. The polymer-only film showed the smallest relative pore resistance and largest relative coating capacitance, while the polymer with added LFPc showed the largest relative pore resistance and smallest relative coating capacitance. With the exception of the LFPc film, after a variable length of weathering, small spots of corrosion appeared on the coated substrates at the indicated time points (dashed lines) in Figure 6.7b. Notably, the LNa film showed corrosion at the earliest time point (1500 h), possibly due to loss of the clay from that film. Additionally, each increase in impedance of the LNa film (2000 and 3500 hours) was accompanied by an increase in $R_{c t}$ which indicated a growing passivating layer of corrosion had filled the pores. Similar observations were made for LAPC at 4500 hours. Small layers of corrosion at the bottom of pore channels offered limited protection, as their resistance was eventually overcome during soaking studies. By all of these EIS measures, LFPC was the only coating that did not develop visible corrosion and had significantly better performance than the other films during accelerated weathering.

Another measure of a film's ability to prevent corrosion is the time constant $\left(T_{\text {coat }}=C_{\text {coat }} \cdot R_{\text {pore }}\right)$, which is reported for each film in Appendix $F$. The $R C$ time constants 
are correlated with the degree of exfoliation of clay (as determined by SAXS models, with the data shown in Table 6.1): the more the clays are exfoliated, the longer are the time constants of the final films. Both initially and throughout weathering, the resinonly film performed the poorest with respect to each circuit element and had the lowest overall barrier properties. Addition of any clay increased the time constant 5-45 times that of the polymer only coating.

\subsubsection{Electrochemical stress index}

Using maximum and minimum values from the time-course EIS spectra of key elements in the EEC in Figure 6.7d, a metric for quantifying the electrochemical stress (ES) index within a film can be estimated, ${ }^{189}$ and is shown in equation 6.1:

$E S=\frac{\log \left[R_{\text {pore }(\max )} / R_{\text {pore }(\min )}\right]+\log \left[R_{c t(\max )} / R_{c t(\min )}\right]+\log \left[C_{\operatorname{coat}(\max )} / C_{c o a t(\min )}\right]+\log \left[C_{d l(\max )} / C_{d l(\min )}\right]}{\log 1 \times 10^{5}}$

In the ES index calculation, a resulting value close to zero indicates low stress while a value of 3 is the theoretical maximum. By this metric, the PVDF resin (without nano-clay additives) had an $\mathrm{ES}=2.66$ after 4500 hours of weathering, which was the largest stress index in the study and makes this coating the least weatherable. Incorporation of nano-clays had positive effects on reducing the stress index of the material: the unmodified clay LNa had an ES $=1.48$ and films with modified clays showed even smaller changes where $E S=0.993$ for $L A P c$ and $E S=0.987$ for LFPc. From these data, incorporation of nano-clays reduces the stress index of this PVDF coating, while the magnitude of that change was dependent on the chemical-modification of the nano-clay. 


\subsection{Photochemical changes of modified nano-clay PVDF composites}

The data presented thus far showed that the addition of chemically-modified nanoclays results in significant improvements on the barrier properties, both short and longterm, of an already highly weatherable coating. In this ATR-FTIR study, we observed that acrylic side-groups from the polymer were lost with weathering, as can be seen in the subtraction spectra of PVDF-acrylic films shown in Figure 6.8 , and that the losses were not equal across all films. It is useful to note that many of the degradation products of acrylic polymers are volatile and thus are not detected, especially near the surface. In the IR spectra, all of the negative peaks are associated with the acrylic portion of the resin while nearly all of the positive peaks are attributed to the PVDF portion of the resin. Specifically, the loss of acrylic is most readily observed in the large negative peak at $1731 \mathrm{~cm}^{-1}$ associated with the carbonyl in the acrylate (a functional group that that is not present in PVDF). Additional loss of aliphatic methyl character was observed at $2989 \mathrm{~cm}^{-1}\left(\mathrm{CH}_{3} V_{\text {as }}\right), 2877 \mathrm{~cm}^{-1}\left(\mathrm{CH}_{3} V_{\mathrm{s}}\right)$, and $1472 / 1435 \mathrm{~cm}^{-1}\left(\mathrm{CH}_{3} \delta_{\text {as }}\right)$ while loss of aliphatic methylene character was observed at $2958 \mathrm{~cm}^{-1}\left(\mathrm{CH}_{2} \mathrm{Vas}_{\mathrm{as}}\right), 2847$ $\mathrm{cm}^{-1}\left(\mathrm{CH}_{2} V_{s}\right)$, and $1450 \mathrm{~cm}^{-1}\left(\mathrm{CH}_{2} \delta\right.$ scissor $)$. The largest change in aliphatic groups occurred with a negative peak at $2926 \mathrm{~cm}^{-1}$ and was assigned as a methylene stretching band. A variety of $C-F_{2}$ stretching vibrations were present from $1300-800 \mathrm{~cm}^{-1}$, all positive in the subtraction spectra. Large positive peaks associated with the PVDF backbone were assigned to the $\mathrm{CH}_{2}$ scissor mode $\left(1400 \mathrm{~cm}^{-1}\right), \mathrm{CF}_{2} / \mathrm{CH}_{2}$ twisting (882 cm $\left.{ }^{1}\right)$ and $\mathrm{CF}_{2} / \mathrm{CH}_{2}$ skeletal $-\beta$ twisting $\left(840 \mathrm{~cm}^{-1}\right)$. The increase in the PVDF vibrational modes indicate that component exists in greater proportions at the surface of the coating. In 
fact, after $4000 \mathrm{~h}$, the ATR-FTIR spectrum indicated that the surfaces of the films without nano-clays were nearly devoid of any acrylic character in the polymer and were

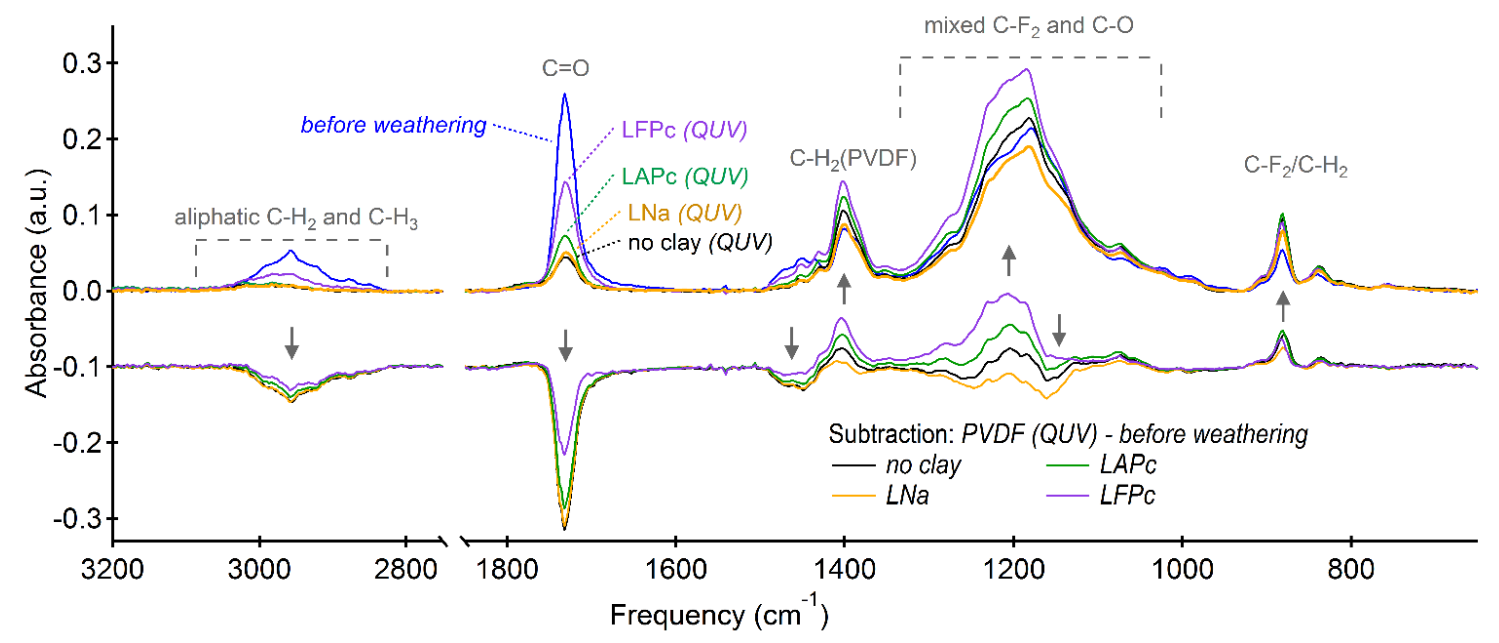

Figure 6.8 FTIR-ATR spectra of coated bronze panels before and after $4000 \mathrm{~h}$ weathering by QUV. The subtraction results were produced from the weathered minus the initial (before weathering) spectrum, and are vertically offset by -0.1 a.u. for visual clarity.

nearly purely PVDF.The smallest difference peaks by ATR-FTIR were observed for the perfluoroalkyl clay composites. It has been hypothesized that smectites can act as free radical quenchers during UV exposure, ${ }^{59}$ which could also explain the reduction in photodegradation observed when clays were incorporated.

Surface ablation of the coatings was confirmed by TF-XRD (data shown in Appendix F, Figure F5), where the spectra show a two important features: (1) a loss of film thickness and (2) surface enrichment of crystalline PVDF. The film that thinned most and showed the greatest increase in PVDF crystallinity after weathering was the polymer only film. TF-XRD data also showed that addition of nano-clays reduced loss and changes in polymer film crystallinity. Material loss can also be monitored by relative changes in film thickness and gloss measurements, data for which are listed in 
Table 6.3 for each coating after weathering by QUV. Addition of the LNa clay did not appreciably affect gloss of the PVDF coating before or after weathering, but slightly prevented film loss over time. LFPc films had the lowest initial gloss compared to all of the other films (having a more matte appearance is often considered to be an aesthetic improvement for coatings, to reduce glare). LFPc films also showed the least change in gloss and the smallest change in film thickness $(<30 \%)$ after weathering. Since the chemical functionality of the APTMS silane is more similar to that of the ester pendant groups in the acrylic resin, modified LAPc clays may be more susceptible to chemical degradation than clays of LFPc. If LAPc loses chemical functionality, the clay could be washed out from the coating matrix, resulting in a loss of the film due to the loss of radical quenchers in the clay.

Table 6.3 Gloss and thickness of the films before and after 4000 hours QUV-B

\begin{tabular}{lccc}
\hline & $\begin{array}{c}\text { Gloss }(\% \mathrm{R}) \\
\text { Initial }\end{array}$ & $\begin{array}{c}\text { Gloss }(\% \Delta \mathrm{R}) \\
\text { QUV } 4 \mathrm{kh}\end{array}$ & $\begin{array}{c}\text { Thickness }(\% \Delta \mathrm{D}) \\
\text { QUV } 4 \mathrm{k} \mathrm{h}\end{array}$ \\
\hline PVDF & $12.6 \pm 1.1$ & $-33.3 \pm 2.9$ & $-36.5 \pm 9.8$ \\
PVDF+LNa & $12.6 \pm 0.3$ & $-35.7 \pm 0.96$ & $-29.4 \pm 8.5$ \\
PVDF+LAPc & $13.2 \pm 0.4$ & $-37.9 \pm 1.2$ & $-43.3 \pm 7.5$ \\
PVDF+LFPc & $9.1 \pm 0.2$ & $-13.2 \pm 0.44$ & $-23.1 \pm 2.1$ \\
\hline
\end{tabular}

It has been observed in the literature that irradiation, including that by UV light, is capable of inducing conformational changes in semi-crystalline fluoropolymers. ${ }^{42,} 190$ Structural changes of the polymer that result in an increase in crystallinity would likely give rise to a film that has excellent barrier properties into the coating, but out of the coating as well. Based on the ATR-FTIR data, the percentage of PVDF at the surface increased to near purity during weathering and the films became more crystalline (XRD data before and after weathering is shown in Figure F5 $_{5}$. The EIS stability of particularly 
the LFPc film during weathering suggests that the films weather into two-phases: 1) microcrystalline PVDF enriched domains and 2) nanocrystalline modified clays, with both phases having electrolyte/corrosive impenetrable crystalline domains.

\subsection{Conclusions}

We have demonstrated that by incorporating chemically-modified nano-clays, the barrier properties of films produced from aqueous dispersions of acrylic-PVDF lattices can be significantly improved over both the short and long term. XPS and Raman data showed that: (1) Laponite clay was covalently-modified with FOTES and cationexchanged with $\mathrm{Pc}$, (2) Grafting efficiency was over 100\%, suggesting that each accessible hydroxyl along the clay rim was covalently bound to one FOTES molecule, (3) the approximately $50 \%$ excess of silanes remaining after washing were covalently cross-linked to other FOTES molecules, and (4) cation exchange with Pc increased the hydrophilicity of the covalently modified clay. SAXS modeling was usefully employed to monitor nanoparticle-latex interactions and particle sizes. Covalently modified nanoclay particles were dispersible in aqueous colloidal suspensions of a highly weatherable resin - and with a much smaller loading weight ( $0.6 \%$ of dry film) than other studies have reported. ${ }^{168-169,174}$

Weathering performance of the nano-composite films was better in every measured parameter than in the films without clay. All composite films studied retained their high performance barrier properties, as measured by EIS and stress index, after a substantial loss of acrylic functionality near the surface and a loss of 10- 
$40 \%$ of film thickness. However, the performance characteristics of each differed considerably. While the PVDF/acrylic waterborne resin is an exceptional barrier top coat with long-term weatherability, its performance can be substantially improved by the inclusion of chemically compatible nano-clays.

What accounts for the better EIS barrier performance of LFPc compared to LAPC composite films during weathering? It seems likely that the interaction between the polymer resin and the organo-functionalized clays may play a substantial role. The latex polymer used in these studies was synthesized in a core-shell synthesis, with both the core and the shell having both, but differing amounts of acrylic and PVDF components. ${ }^{48,} 166$ To enable dispersion in aqueous media, the shell has a greater percentage of acrylic (hydrophilic) than the core does. Given that the best barrier properties were seen in films with added LFPc, it is possible that the LFPc clays facilitate the formation of an interpenetrating polymer network better than LAPC.

Good stability (chemical and mechanical) was observed by the low stress-index of chemically modified clays in the PVDF/acrylic films. The LNa and LFPc clays had the greatest degree of exfoliation, the most distant scattering neighbor in both water and the latex resin, and the longest time constants for much of the weathering time. However, the unmodified LNa composite film had a lower film resistance and higher coating capacitance than the LFPc composite film. One explanation for the LNa composite film's diminished barrier properties despite having a longer time constant, may arise from the microstructural arrangement of the clays in the interstitial spaces of the wet resin during film formation. Evidence suggests that while LNa was exfoliated 
completely in water, there was little chemical compatibility with the latex particles in the film, which can disrupt coalescence of latex particles during drying. The result would be larger voids between latex particles where migrating ion species are retained, as was indeed suggested by the water-whitening studies. Chemical modification resulted in clays that remained exfoliated and improved their dispersability in this highly weatherable resin. It is likely that the combination of the microstructural arrangement of clay particles, chemical compatibility between FOTES clays and the PVDF portion of the resin, and the inert nature of perfluoroalkyl and PVDF functionalities account for the superior corrosion resistance of the LFPc composite films throughout weathering. 


\section{Final Thoughts}

Working in the unique framework of cultural heritage conservation, a vast amount of insight was shed onto the working properties of well-loved coatings. Waxes were found to be insufficient barrier coatings for nearly every sample studied, likely due to fundamental flaws in the film structure. Similarly, waterborne emulsion coatings were investigated and found to have particular weaknesses for water whitening and slow coalescence of films, both of which were attributed to uptake caused by residual additives in the film. WL81 would not be recommended for use on cultural heritage pieces weathered outdoors due to its extreme sensitivity to UV degradation and rapid failure. Surprisingly Paraloid B-44 coatings typically did not survive beyond 1250-1500 hours in the QUV, which is only slightly longer than some reported waxes. Similar to other results, Incralac stayed intact during outdoor weathering of three years with decent barrier properties on bronze and patinated steel. None of the traditional materials performed better than the annealed PVDF ARC and FMA coatings in parameters such as protective lifetime, workability, and gloss.

Chemically compatible clays were successfully functionalized with silanes APTMS and FOTES, monomers similar to those in the PVDF/acrylic latex resin. The organic nano-clays were stable for weeks longer in solution upon cationic exchange with phosphoryl choline than with the use of a peptizing agent. Eventually, clear, stable sols of modified clays were produced for addition into the FMA resin as an additive before spraying. These suspensions were incorporated into resins from which high- 
impedance coatings were sprayed. These coatings were tested alongside PNCs with non-functionalized clay as well as the FMA top-coat without clay; and, were consistently found superior for protection of the underlying metal substrate. PNC coatings with LFPc in particular excelled in each TRACE requirement listed in Chapter 5 and is recommended for future consideration in protection of cultural heritage items.

Materials like LFPc should be investigated further as their unique properties are well suited to tackle some unique goals of conservation of heritage. Evidence presented in Chapter 6 of long lifetimes, small amounts of short-range order, reduced masstransport and whitening were incongruous with surface enrichment of PVDF from acrylic degradation. Surface-enrichment of PVDF indicates degradation with weathering, but only increases in targeted barrier properties. At the end of the weathering studies performed here, the B44 primer coat was intact and flexible under the nanocomposite films while the 70:30 PVDF:Acrylic (ARC) coating without clay was much was more brittle and had little adhesion to the metal surface if stressed. What keeps the $\mathrm{B} 44$ primer coating intact and pliable under the PNC for twice its protective lifetime alone? Functions of clay could be to (1) scatter incoming UV radiation, minimizing the amount getting to the base coat and (2) minimize mass transport out of the film during photodegradation, particularly of volatile species which cannot transport through crystalline domains and may instead recombine if they have active terminal groups. Either results in a decrease in photodegradation of the coating with more mass, gloss, and ideally barrier properties for longer. Future considerations for this project would immediately take the PVDF nanocomposites with functionalized 
nano-clays outside for weathering. While the QUV is useful for material development, the intense and unnatural UV-B radiation can only provide so much insight to how a coating will survive in rainy conditions outdoors. The LFPC PNC discussed in Chapter 6 should perform even better outdoors, where surface ablation of the acrylic part of the blend would be slower. 


\section{References}

1. Westing, A. H., From Environmental to Comprehensive Security. Springer: 2013.

2. Schmitt, G., Global needs for knowledge dissemination, research, and development in materials deterioration and corrosion control. New York, NY: The World Corrosion Organization 2009.

3. Milne, A., Economics and the environment: the role of coatings. In The Chemistry and Physics of Coatings, Marrion, A., Ed. The Royal Society of Chemistry: 2004, pp 1-7.

4. Elder), P. t., Naturalis historia. C.E. 77-79; Vol. Book XXI.

5. Bastidas, D. M.; Criado, M.; Fajardo, S.; La Iglesia, V. M.; Cano, E.; Bastidas, J. M., Copper deterioration: causes, diagnosis and risk minimisation. International Materials Reviews 2010, 55 (2), 99-127.

6. Curkovic, H. O.; Kosec, T.; Legat, A.; Stupnisek-Lisac, E., Improvement of corrosion stability of patinated bronze. Corrosion Engineering Science and Technology 2010, 45 (5), 327-333.

7. Kosec, T.; Curkovic, H. O.; Legat, A., Investigation of the corrosion protection of chemically and electrochemically formed patinas on recent bronze. Electrochimica Acta 2010, 56 (2), 722-731.

8. Carbonini, P.; Monetta, T.; Nicodemo, L.; Mastronardi, P.; Scatteia, B.; Bellucci, F., Electrochemical characterisation of multilayer organic coatings. Progress in Organic Coatings 1996, 29 (1-4), 13-20.

9. Bierwagen, G., The physical chemistry of organic coatings revisited: viewing coatings as a materials scientist. Journal of Coatings Technology and Research 2008, $5(2), 133-155$.

10. Cano, E.; Bastidas, D. M.; Argyropoulos, V.; Fajardo, S.; Siatou, A.; Bastidas, J.; Degrigny, $C$., Electrochemical characterization of organic coatings for protection of historic steel artefacts. Journal of Solid State Electrochemistry 2010, 14 (3), 453-463.

11. Clare, T. L.; Lins, P. A. In Evaluation of fluorinated protective coatings for outdoor metals, Proceedings of Metal 07: Interim Meeting of the ICOM-CC Metal WG Rijksmuseum, Amsterdam, Degrigny, C.; Lang, R. V.; Joosten, I.; Ankersmith, B., Eds. ICOM, 2007: Rijksmuseum, Amsterdam, 2007; pp 83-87.

12. Cappitelli, F.; Sorlini, C., Microorganisms Attack Synthetic Polymers in Items Representing Our Cultural Heritage. Applied and Environmental Microbiology 2008, $74(3), 564-569$.

13. Miliani, C.; Ombelli, M.; Morresi, A.; Romani, A., Spectroscopic study of acrylic resins in solid matrices. Surface and Coatings Technology 2002, 151-152, 276-280.

14. de Silva, M.; Henderson, J., Sustainability in conservation practice. Journal of the Institute of Conservation 2011, 34 (1), 5-15.

15. Russell, A.; Milford, J.; Bergin, M. S.; McBride, S.; McNair, L.; Yang, Y.; Stockwell, W. R.; Croes, B., Urban Ozone Control and Atmospheric Reactivity of Organic Gases. Science 1995, 269 (5223), 491-495. 
16. Ogawa, M.; Saito, K.; Sohmiya, M., A controlled spatial distribution of functional units in the two dimensional nanospace of layered silicates and titanates. Dalton Transactions 2014, 43 (27), 10340-10354.

17. Gebhard, M. S., The challenge of achieving traditional exterior durability performance in low VOC architectural coatings. Macromolecular Symposium 2002, 187, 771-780.

18. Wheeler, O. L.; Jaffe, H. L.; Wellman, N., A mechanism of film formation of polyvinyl acetate emulsions. Official Digest, Federation of Paint and Varnish 1954, 26, 1239 .

19. ICOM-CC Terminology to characterize the conservation of tangible cultural heritage. http://www.icom-cc.org/54/documents/terminology-to-characterize-theconservation-of-tangible-cultural-heritage-english/?id=368 (accessed April 16).

20. Cano, E.; Lafuente, D.; Bastidas, D. M., Use of EIS for the evaluation of the protective properties of coatings for metallic cultural heritage: a review. Journal of Solid State Electrochemistry 2010, 14, 381-391.

21. Kosec, T.; Legat, A.; Milosev, I., The comparison of organic protective layers on bronze and copper. Progress in Organic Coatings 2010, 69 (2), 199-206.

22. Robeson, L. M.; Hyder, I. Z., Novel water sorption behavior of emulsion blends. Journal of Applied Polymer Science 2003, 90 (4), 933-939.

23. Shay, G. D.; Olesen, K. R.; Stallings, J. L., Predicting the water-sensitivity of filmforming coatings additives by water vapor sorption: with application to thickeners and rheology modifiers.(Cover Story). Journal of Coatings Technology and Research 1996, v68 (n854), p51(13).

24. Arnold, C.; Thalmann, F.; Marques, C.; Marie, P.; Holl, Y., Surfactant Distribution in Waterborne Acrylic Films. 1. Bulk Investigation. Journal of Physical Chemistry $B$ 2010, 114 (28), 9135-9147.

25. Brown, R. F. G., Additives in coatings - a necessary evil? In Additives in Water-borne Coatings, Davison, G.; Lane, B., Eds. The Royal Society of Chemistry: 2003, pp 1-6.

26. Delucchi, M.; Trombetta, T.; Ricotti, R.; Cerisola, G.; Turri, S., Study of metal protective hydrophobic coatings from waterborne polyurethane ionomers. Journal of Applied Electrochemistry 2009, 39 (11), 2107-2113.

27. Scalarone, D.; Lazzari, M.; Castelvetro, V.; Chiantore, O., Surface Monitoring of Surfactant Phase Separation and Stability in Waterborne Acrylic Coatings. Chemistry of Materials 2007, 19 (25), 6107-6113.

28. Curkovic, H. O.; Kosec, T.; Legat, A.; Stupnisek-Lisac, E., Improvement of corrosion stability of patinated bronze. Corros. Eng. Sci. Techn. 2010, 45 (5), 327-333.

29. El-Taib Heakal, F.; Haruyama, S., Impedance studies of the inhibitive effect of benzotriazole on the corrosion of copper in sodium chloride medium. Corrosion Science 1980, 20 (7), 887-898.

30. Rahmouni, K.; Takenouti, H.; Hajjaji, N.; Srhiri, A.; Robbiola, L., Protection of ancient and historic bronzes by triazole derivatives. Electrochimica Acta 2009, 54 (22), 5206-5215. 
31. Sease, C., Benzotriazole: A Review for Conservators. Studies in Conservation 1978, $23(2), 76-85$.

32. Steward, P. A.; Hearn, J.; Wilkinson, M. C., An overview of polymer latex film formation and properties. Advances in Colloid and Interface Science 2000, 86 (3), 195-267.

33. Le Pen, C.; Lacabanne, C.; Pébère, N., Characterisation of water-based coatings by electrochemical impedance spectroscopy. Progress in Organic Coatings 2003, 46 (2), 77-83.

34. Routh, A. F.; Russel, W. B., A Process Model for Latex Film Formation: Limiting Regimes for Individual Driving Forces. Langmuir 1999, 15 (22), 7762-7773.

35. Vanderhoff, J. W.; Bradford, E. B.; Carrington, W. K., The transport of water through latex films. Journal of Polymer Science, Part C: Polymer Symposia 1973, 41 (1), 155-174.

36. Vanderhoff, J. W., Mechanism of film formation of latices. British Polymer Journal 1970, 2 (3), 161-173.

37. Swartz, N. A.; Clare, T. L., Understanding the differences in film formation mechanisms of two comparable solvent based and water-borne coatings on bronze substrates by electrochemical impedance spectroscopy. Electrochimica Acta 2012, 62, 199-206.

38. Fekete, E.; Lengyel, B., Accelerated testing of waterborne coatings. Progress in Organic Coatings 2005, 54 (3), 211-215.

39. Procopio, L. J.; Larson, G. R.; Rosano, W. J., Low-VOC waterborne coatings for use in industrial maintenance painting. JCT Coatingstech 2007, 4 (2), 50-59.

40. Allen, N. S.; Regan, C. J.; Dunk, W. A. E.; Mclntyre, R.; Johnson, B., Aspects of the thermal, photodegradation and photostabilisation of water-borne fluorinatedacrylic coating systems. Polymer Degradation and Stability 1997, 58 (1-2), 149-157.

41. Chiantore, O.; Lazzari, M., Photo-oxidative stability of paraloid acrylic protective polymers. Polymer 2001, 42 (1), 17-27.

42. Gu, X.; Michaels, C. A.; Nguyen, D.; Jean, Y. C.; Martin, J. W.; Nguyen, T., Surface and interfacial properties of PVDF/acrylic copolymer blends before and after UV exposure. Applied Surface Science 2006, 252 (14), 5168-5181.

43. Castela, A. S. L.; Simões, A. M.; Ferreira, M. G. S., E.I.S. evaluation of attached and free polymer films. Progress in Organic Coatings 2000, 38 (1), 1-7.

44. Agarwal, N.; Farris, R. J., Water absorption by acrylic-based latex blend films and its effect on their properties. Journal of Applied Polymer Science 1999, 72 (11), 14071419.

45. Strafford, O. A.; Hinderliter, B. R.; Croll, S. G., Electrochemical impedance spectroscopy response of water uptake in organic coatings by finite elemnt methods. Electrochimica Acta 2006, 52, 1339-1348.

46. Keddie, J. L.; Routh, A. F., Fundamentals of latex film formation. Springer: Dordrecht [etc.], 2010.

47. Wood, K. A.; Cypcar, C.; Hedhli, L., Predicting the exterior durability of new fluoropolymer coatings. Journal of Fluorine Chemistry 2000, 104 (1), 63-71. 
48. Iezzi, R. A.; Gaboury, S.; Wood, K., Acrylic-fluoropolymer mixtures and their use in coatings. Progress in Organic Coatings 2000, 40 (1-4), 55-60.

49. Deflorian, F.; Fedrizzi, L.; Lenti, D.; Bonora, P. L., On the corrosion protection properties of fluoropolymer coatings. Progress in Organic Coatings 1993, 22 (1-4), 39-53.

50. Ciardelli, F.; Aglietto, M.; Montagnini di Mirabello, L.; Passaglia, E.; Giancristoforo, S.; Castelvetro, V.; Ruggeri, G., New fluorinated acrylic polymers for improving weatherability of building stone materials. Progress in Organic Coatings 1997, 32 (14), 43-50.

51. He, L.; Liang, J.; Zhao, X.; Li, W.; Luo, H., Preparation and comparative evaluation of well-defined fluorinated acrylic copolymer latex and solution for ancient stone protection. Progress in Organic Coatings 2010, 69 (4), 352-358.

52. Shi, A.; Koka, S.; Ullett, J., Performance evaluation on the weathering resistance of two USAF coating systems (standard 85285 topcoat versus fluorinated APC topcoat) via electrochemical impedance spectroscopy. Progress in Organic Coatings 2005, 52 (3), 196-209.

53. Bierwagen, G.; Shedlosky, T. J.; Stanek, K., Developing and testing a new generation of protective coatings for outdoor bronze sculpture. Progress in Organic Coatings 2003, 48 (2-4), 289-296.

54. Clare, T. L.; Swartz, N. A., Characterization of high performance protective coatings for use on culturally significant works. In Intelligent Coatings for Corrosion Control, Tiwari, A.; Hihara, L.; Rawlins, J., Eds. Butterworth-Heinemann: Elsevier, 2014; Chapter 19, pp 641-671.

55. Croll, S.; Fernando, D.; Skaja, A., Mechanical property changes and degradation during accelerated weathering of polyester-urethane coatings. JCT Research 2006/01//, 2006, p 41+.

56. Deflorian, E.; Fedrizzi, L.; Bonora, P. L., Study of the Water Barrier Properties of Paints After Natural and Accelerated Photooxidative Degradation. In Organic Coatings for Corrosion Control, American Chemical Society: 1998; Chapter 8, pp 92105.

57. Allen, N. S., Recent advances in the photo-oxidation and stabilization of polymers. Chemical Society Reviews 1986, 15 (3), 373-404.

58. Langmuir, I., The Role of Attractive and Repulsive Forces in the Formation of Tactoids, Thixotropic Gels, Protein Crystals and Coacervates. The Journal of Chemical Physics 1938, 6 (12), 873-896.

59. Essawy, H. A.; Abd El-Wahab, N. A.; Abd El-Ghaffar, M. A., PVC-laponite nanocomposites: Enhanced resistance to UV radiation. Polymer Degradation and Stability 2008, 93 (8), 1472-1478.

6o. Bippus, L.; Jaber, M.; Lebeau, B., Laponite and hybrid surfactant/laponite particles processed as spheres by spray-drying. New Journal of Chemistry 2009, 33 (5), 11161126.

61. Ruzicka, B.; Zaccarelli, E., A fresh look at the Laponite phase diagram. Soft Matter 2011, $7(4), 1268-1286$. 
62. Daniel, L. M.; Frost, R. L.; Zhu, H. Y., Edge-modification of laponite with dimethyloctylmethoxysilane. Journal of Colloid and Interface Science 2008, 321 (2), 302-309.

63. Wang, J.; Wheeler, P. A.; Jarrett, W. L.; Mathias, L. J., Synthesis and characterization of dual-functionalized laponite clay for acrylic nanocomposites. Journal of Applied Polymer Science 2007, 106 (3), 1496-1506.

64. Bathfield, M.; D'Agosto, F.; Spitz, R.; Charreyre, M.-T.; Delair, T., Versatile Precursors of Functional RAFT Agents. Application to the Synthesis of Bio-Related End-Functionalized Polymers. Journal of the American Chemical Society 2006, 128 (8), 2546-2547.

65. Negrete-Herrera, N.; Letoffe, J.-M.; Putaux, J.-L.; David, L.; Bourgeat-Lami, E., Aqueous Dispersions of Silane-Functionalized Laponite Clay Platelets. A First Step toward the Elaboration of Water-Based Polymer/Clay Nanocomposites. Langmuir 2004, 20 (5), 1564-1571.

66. Michot, L. J.; Bihannic, I.; Maddi, S.; Funari, S. S.; Baravian, C.; Levitz, P.; Davidson, P., Liquid crystalline aqueous clay suspensions. Proceedings of the National Academy of Science 2006, 103 (44), 16101-16104.

67. Murray, J. N., Electrochemical test methods for evaluating organic coatings on metals: an update. Part III: Multiple test parameter measurements. Progress in Organic Coatings 1997, 31 (4), 375-391.

68. Hinderliter, B. R.; Croll, S. G.; Tallman, D. E.; Su, Q.; Bierwagen, G. P., Interpretation of EIS data from accelerated exposure of coated metals based on modeling of coating physical properties. Electrochimica Acta 2006, 51 (21), 45054515.

69. Bierwagen, G. P.; Wang, X.; Tallman, D. E., In situ study of coatings using embedded electrodes for ENM measurements. Progress in Organic Coatings 2003, $46(3), 163-175$.

70. Chércoles Asensio, R.; San Andrés Moya, M.; de la Roja, J.; Gómez, M., Analytical characterization of polymers used in conservation and restoration by ATR-FTIR spectroscopy. Analytical and Bioanalytical Chemistry 2009, 395 (7), 2081-2096.

71. Clare, T. L.; Lins, A., LG25-08-0071-08: Federally compliant protective clear coatings for metals. Institute of Museum and Library Services: Portland, OR, 2008.

72. le Roux, J. H., Investigation of the structure of Fischer-Tropsch oxidized waxes by means of X-ray diffraction, Nuclear magnetic-resonance and Infrared spectroscopy. South African Journal of Science 1979, 75 (8), 356-361.

73. Petersson, M.; Gustafson, I.; Stading, M., Comparison of microstructural and physical properties of two petroleum waxes. Journal of Materials Science 2008, 43 (6), 1869-1879.

74. Shen, M.; Hansen, W. N.; Romo, P. C., Thermal Expansion of the Polyethylene Unit Cell. Journal of Chemical Physics 1969, 51 (1), 425-430.

75. Dorset, D. L., Chain Length Distribution and the Lamellar Crystal Structure of a Paraffin Wax. Journal of Physical Chemistry B 2000, 104 (35), 8346-8350.

76. Considine, B. B.; Wolfe, J.; Posner, K.; Bouchard, M., Conserving Outdoor Sculpture: The Stark Collection at the Getty Center. Getty Conservation Institute: 2010. 
77. Christman, B., Twenty-Five Years After the Bomb: Maintaining Cleveland's the Thinker. Journal of the American Institute for Conservation 1998, 37 (2), 173-186.

78. Wang, L.; Ando, S.; Ishida, Y.; Ohtani, H.; Tsuge, S.; Nakayama, T., Quantitative and discriminative analysis of carnauba waxes by reactive pyrolysis-GC in the presence of organic alkali using a vertical microfurnace pyrolyzer. J. Anal. Appl. Pyrolysis 2001, 58-59 (0), 525-537.

79. Carol A. Grissom, N. G., Corey Smith Riley, and A. Elena Charola, Evaluation of Coating Performance on Silver Exposed to Hydrogen Sulfide. Journal of the American Institute for Conservation 2013, 52 (2), 82-96.

80. Jaeger, T., Short Communication Removal of Paraffin Wax in the Re-treatment of Archaeological Iron. Journal of the American Institute for Conservation 2008, 47 (3), 217-223.

81. Moffett, D. L., Wax Coatings on Ethnographic Metal Objects: Justifications for Allowing a Tradition to Wane. Journal of the American Institute for Conservation 1996, 35 (1), 1-7.

82. Otieno-Alego, V.; Heath, G.; Hallam, D.; Creagh, D. In Electrochemical evaluation of the anti-corrosion performance of waxy coatings for outdoor bronze conservation, Conférence internationale sur la conservation des métaux, 1998; pp 309-314.

83. le Roux, J. H., Fischer-tropsch waxes. II. Crystallinity and physical properties. Journal of Applied Chemistry 1969, 19 (3), 86-88.

84. Price, C.; Hallam, D.; Heath, G.; Creagh, D.; Ashton, J. In An electrochemical study of waxes for bronze sculpture, Proceedings of Metal 98: International Conference on Metals Conservation, Figanières-Draguignan, France, Mourey, W.; Robbiola, L., Eds. James \& James: Figanières-Draguignan, France, 1997; pp 233-241.

85. Luongo, J. P., Infrared study of oxygenated groups formed in polyethylene during oxidation. Journal of Polymer Science 1960, 42 (139), 139-150.

86. Heacock, J. F.; Mallory, F. B.; Gay, F. P., Photodegradation of polyethylene film. Journal of Polymer Science Part A: Polymer Chemistry 1968, 6 (10), 2921-2934.

87. Kaplan, M. L.; Kelleher, P. G., On the mechanism of polyethylene photodegradation: The oxidation of terminal olefins and saturated centers with singlet oxygen. Journal of Polymer Science Part B: Polymer Physics 1971, 9 (8), 565568.

88. Mansfeld, F., Use of electrochemical impedance spectroscopy for the study of corrosion protection by polymer coatings. Journal of Applied Electrochemistry 1995, 25 (3), 187-202.

89. Swartz, N. A.; Wood, K. A.; Clare, T. L., Characterizing and improving performance properties of thin solid films produced by weatherable water-borne colloidal suspensions on bronze substrates. Progress in Organic Coatings 2012, 75 (3), 215223.

90. Amand, S.; Musiani, M.; Orazem, M. E.; Pébère, N.; Tribollet, B.; Vivier, V., Constant-phase-element behavior caused by inhomogeneous water uptake in anticorrosion coatings. Electrochimica Acta 2013, 87 (0), 693-700. 
91. Hernandez, R. d. P. B.; Aoki, I. V.; Tribollet, B.; de Melo, H. G., Electrochemical impedance spectroscopy investigation of the electrochemical behaviour of copper coated with artificial patina layers and submitted to wet and dry cycles. Electrochimica Acta 2011, 56 (7), 2801-2814.

92. Otieno-Alego, V.; Creagh, D.; Hallam, D.; Viduka, A.; Heath, G., In Situ and Laboratory Studies of the Ageing of Protective Wax Coatings on Metal Surfaces of Museum Objects and Outdoor Statues. In Ageing Studies and Lifetime Extension of Materials, Mallinson, L. G., Ed. Springer US: 2001; Chapter 67, pp 609-618.

93. Ellingson, L. A.; Shedlosky, T. J.; Bierwagen, G. P.; Rie, E. R. d. I.; Brostoff, L. B., The Use of Electrochemical Impedance Spectroscopy in the Evaluation of Coatings for Outdoor Bronze. Studies in Conservation 2004, 49 (1), 53-62.

94. Otmačić Curkovića, H.; Kosec, T.; Marušića, K.; Legat, A., An electrochemical impedance study of the corrosion protection of artificially formed patinas on recent bronze. Electrochimica Acta 2012, 83, 28-39.

95. Hirschorn, B.; Orazem, M. E.; Tribollet, B.; Vivier, V.; Frateur, I.; Musiani, M., Determination of effective capacitance and film thickness from constant-phaseelement parameters. Electrochimica Acta 2010, 55 (21), 6218-6227.

96. Hsu, C.; Mansfeld, F., Technical note: concerning the conversion of the constant phase element parameter Yo into a capacitance. Corrosion 2001, 57 (9), 747-748.

97. Reynhardt, E. C., NMR investigation of Fischer-Tropsch waxes. II. Hard wax. Journal of Physics D: Applied Physics 1985, 18 (6), 1185.

98. Pu, G.; Severtson, S. J., Dependency of Contact Angle Hysteresis on Crystallinity for n-Alkane Substrates. Journal of Physical Chemistry C 2009, 113 (16), 6673-668o.

99. Trozzolo, A. M.; Winslow, F. H., A Mechanism for the Oxidative Photodegradation of Polyethylene. Macromolecules 1968, 1 (1), 98-100.

100. Schrenk, J. L., Corrosion and Past"Protective "Treatments of the Benin "Bronzes" in the National Museum of African Art. MRS Online Proceedings Library 1990, 185, null-null.

101. Luyt, A. S.; Geethamma, V. G., Effect of oxidized paraffin wax on the thermal and mechanical properties of linear low-density polyethylene-layered silicate nanocomposites. Polymer Testing 2007, 26 (4), 461-470.

102. Bacon, R. C.; Smith, J. J.; Rugg, F. M., Electrolytic Resistance in Evaluating Protective Merit of Coatings on Metals. Industrial and Engineering Chemistry 1948, $40(1), 161-167$.

103. Amirudin, A.; Thieny, D., Application of electrochemical impedance spectroscopy to study the degradation of polymer-coated metals. Progress in Organic Coatings 1995, 26 (1), 1-28.

104. Bieleman, J.; Hajas, J.; Dören, K., Flow-Levelling and Coalescing Agents. In Additives for Coatings, Wiley-VCH Verlag GmbH: 2007, pp 163-200.

105. Brown, G. L., Formation of films from polymer dispersions. Journal of Polymer Science 1956, 22 (102), 423-434.

106. Dobler, F.; Holl, Y., Mechanisms of latex film formation. Trends in Polymer Science $1996,4(5), 145-151$. 
107. Keddie, J. L.; Routh, A. F., Molecular Diffusion Across Particle Boundaries. In Fundamentals of Latex Film Formation, Springer Netherlands: 2010, pp 151-183.

108. Dobler, F.; Holl, Y., Mechanisms of particle deformation during latex film formation. In Film Formation in Waterborne Coatings, American Chemical Society: 1996, pp 22-43.

109. Sheetz, D. P., Formation of films by drying of latex. Journal of Applied Polymer Science 1965, 9 (11), 3759-3773.

110. Butler, L. N.; Fellows, C. M.; Gilbert, R. G., Effect of surfactants used for binder synthesis on the properties of latex paints. Progress in Organic Coatings 2005, 53 (2), 112-118.

111. Deflorian, F.; Fedrizzi, L.; Rossi, S.; Bonora, P. L., Organic coating capacitance measurement by EIS: ideal and actual trends. Electrochimica Acta 1999, 44 (24), 4243-4249.

112. Loveday, D.; Peterson, P.; Rodgers, B., Evaluation of Organic Coatings with Electrochemical Impedance Spectroscopy: Application of EIS to Coatings. JCT CoatingsTech 2004, 1 (10), 88.

113. Marusic, K.; Otmacic-Curkovic, H.; Horvat-Kurbegovic, S.; Takenouti, H.; Stupnisek-Lisac, E., Comparative studies of chemical and electrochemical preparation of artificial bronze patinas and their protection by corrosion inhibitor. Electrochimica Acta 2009, 54 (27), 7106-7113.

114. Le Pen, C.; Vereecken, J., Effect of curing on the corrosion protective properties of thin organosilane films on aluminium. Journal of Applied Electrochemistry 2005, 35 (12), 1303-1309.

115. Freger, V., Diffusion impedance and equivalent circuit of a multilayer film. Electrochemistry Communications 2005, 7 (9), 957-961.

116. Vyas, R. N.; Li, K.; Wang, B., Modifying Randles Circuit for Analysis of Polyoxometalate Layer-by-Layer Films. Journal of Physical Chemistry B 2010, 114 (48), 15818-15824.

117. Jüttner, K., Electrochemical impedance spectroscopy (EIS) of corrosion processes on inhomogeneous surfaces. Electrochimica Acta 1990, 35 (10), 1501-1508.

118. Cao-Paz, A.; Covelo, A.; Farina, J.; Novoa, X. R.; Perez, C.; Rodriguez-Pardo, L., Ingress of water into organic coatings: Real-time monitoring of the capacitance and increase in mass. Progress in Organic Coatings 2010, 69 (2), 150-157.

119. Yang, D. K.; Koros, W. J.; Hopfenberg, H. B.; Stannett, V. T., The effects of morphology and hygrothermal aging on water sorption and transport in Kapton ${ }^{\circledR}$ polyimide. Journal of Applied Polymer Science 1986, 31 (6), 1619-1629.

120. Han, H.; Gryte, C. C.; Ree, M., Water diffusion and sorption in films of highperformance poly( $4,4^{\prime}$-oxydiphenylene pyromellitimide): effects of humidity, imidization history and film thickness. Polymer 1995, 36 (8), 1663-1672.

121. Sacher, E.; Susko, J. R., Water permeation of polymer films. I. Polyimide. Journal of Applied Polymer Science 1979, 23 (8), 2355-2364. 
122. Cheever, G. D.; Malinski, T.; Ngo, P.-A. P.; Ottaviani, R. A., Development of a predictive model for the changes in roughness that occurs during the painting process. Journal of Coatings Technology 1993, 65 (821), $29+$.

123. Del Grosso Destreri, M.; Vogelsang, J. r.; Fedrizzi, L.; Deflorian, F., Water up-take evaluation of new waterborne and high solid epoxy coatings. Part II: electrochemical impedance spectroscopy. Progress in Organic Coatings 1999, 37 (12), 69-81.

124. Brasher, D. M.; Kingsbury, A. H., Electrical measurements in the study of immersed paint coatings on metal. I. Comparison between capacitance and gravimetric methods of estimating water-uptake. Journal of Applied Chemistry 1954, 4 (2), 6272.

125. Vlasak, R.; Klueppel, I.; Grundmeier, G., Combined EIS and FTIR-ATR study of water uptake and diffusion in polymer films on semiconducting electrodes. Electrochimica Acta 2007, 52 (28), 8075-8080.

126. Kiil, S., Drying of latex films and coatings: Reconsidering the fundamental mechanisms. Progress in Organic Coatings 2006, 57 (3), 236-250.

127. Pérez, C.; Collazo, A.; Izquierdo, M.; Merino, P.; Nóvoa, X. R., Characterisation of the barrier properties of different paint systems: Part II. Non-ideal diffusion and water uptake kinetics. Progress in Organic Coatings 1999, 37 (3-4), 169-177.

128. Bindschaedler, C.; Gurny, R.; Doelker, E., Influence of Emulsifiers on Film Formation from Cellulose-Acetate Latexes Experimental-Study of PhaseSeparation Phenomena Due to Sodium Dodecyl-Sulfate .1. Journal of Applied Polymer Science 1987, 34 (8), 2631-2647.

129. Colombini, D.; Hassander, H.; Karlsson, O. J.; Maurer, F. H. J., Effects of thermal annealing on the viscoelastic properties and morphology of bimodal hard/soft latex blends. Journal of Polymer Science Part B: Polymer Physics 2005, 43 (17), 2289-2306.

130. Mahdavian, M.; Attar, M. M., Another approach in analysis of paint coatings with EIS measurement: Phase angle at high frequencies. Corrosion Science 2006, 48 (12), 4152-4157.

131. O'Donoghue, M.; Garrett, R.; Datta, V.; Roberts, P.; Aben, T., Electrochemical Impedance Spectroscopy: Testing Coatings for Rapid Immersion Service. Materials Performance 2003, pp 36-41.

132. Vogelsang, J.; Strunz, W., New interpretation of electrochemical data obtained from organic barrier coatings. Electrochimica Acta 2001, 46 (24-25), 3817-3826.

133. Bierwagen, G. P., Film coating technologies and adhesion. Electrochimica Acta 1992, 37 (9), 1471-1478.

134. Ovid'ko, I., Defects and Deformation Mechanisms in Nanostructured Coatings. In Nanostructured Coatings, Cavaleiro, A.; Hosson, J. T. M., Eds. Springer New York: 2006, pp 78-108.

135. Rammelt, U.; Reinhard, G., Characterization of active pigments in damage of organic coatings on steel by means of electrochemical impedance spectroscopy. Progress in Organic Coatings 1994, 24 (1-4), 309-322. 
136. Leidheiser, H., Electrical and electrochemical measurements as predictors of corrosion at the metal--organic coating interface. Progress in Organic Coatings 1979, $7(1), 79-104$.

137. Lin, F.; Meier, D. J., A Study of Latex Film Formation by Atomic Force Microscopy. 1. A Comparison of Wet and Dry Conditions. Langmuir 1995, 11 (7), 2726-2733.

138. Lin, F.; Meier, D. J., A Study of Latex Film Formation by Atomic Force Microscopy. 2. Film Formation vs Rheological Properties: Theory and Experiment. Langmuir 1996, 12 (11), 2774-2780.

139. Bierwagen $G$, e. a. In Electrochemical Studies of the protection of bronzes from corrosion by organic coatings, Proceedings of Metal 2001: International Conference on Metals Conservationt, Santiago, Chile, April 2001; MacLeod, I.; Theile, J.; Degriny, C., Eds. Western Australian Museum, Welshpool: Santiago, Chile, 2004; pp 291-296.

140. Bajaj, P.; Goyal, M.; Chavan, R. B., Thermal-Behavior of Methacrylic-Acid Ethyl Acrylate Copolymers. Journal of Applied Polymer Science 1994, 51 (3), 423-433.

141. Allen, N. S.; Parker, M. J.; Regan, C. J.; McIntyre, R. B.; Dunk, W. A. E., THE DURABILITY OF WATER-BORNE ACRYLIC COATINGS. Polymer Degradation and Stability 1995, $47(1), 117-127$.

142. Melo, M. J.; Bracci, S.; Camaiti, M.; Chiantore, O.; Piacenti, F., Photodegradation of acrylic resins used in the conservation of stone. Polymer Degradation and Stability 1999, 66 (1), 23-30.

143. Chiantore, O.; Trossarelli, L.; Lazzari, M., Photooxidative degradation of acrylic and methacrylic polymers. Polymer 2000, 41 (5), 1657-1668.

144. Lazzari, M.; Aglietto, M.; Castelvetro, V.; Chiantore, O., Photochemical stability of partially fluorinated acrylic protective coatings. 2. Copolymers of $1 \mathrm{H}, 1 \mathrm{H}, 2 \mathrm{H}, 2 \mathrm{H}$ perfluorodecyl methacrylate with unfluorinated acrylic esters. Chemistry of Materials 2001, 13 (9), 2843-2849.

145. Lazzari, M.; Chiantore, O.; Castelvetro, V., Photochemical stability of partially fluorinated acrylic protective coatings. Part 3. Copolymers of $1 \mathrm{H}, 1 \mathrm{H}, 2 \mathrm{H}, 2 \mathrm{H}$ perfluorodecyl acrylate and 2,2,2-trifluoroethyl methacrylate with butyl methacrylate. Polym. Int. 2001, 50 (8), 863-868.

146. Ncube, E.; Meincken, M., Surface characteristics of coated soft- and hardwoods due to UV-B ageing. Applied Surface Science 2010, 256 (24), 7504-7509.

147. Lazzari, M.; Scalarone, D.; Malucelli, G.; Chiantore, O., Durability of acrylic films from commercial aqueous dispersion: Glass transition temperature and tensile behavior as indexes of photooxidative degradation. Progress in Organic Coatings 2011, $70(2-3), 116-121$.

148. Lazzari, M.; Chiantore, O., Thermal-ageing of paraloid acrylic protective polymers. Polymer 2000, 41 (17), 6447-6455.

149. Soeriyadi, A. H.; Trouillet, V.; Bennet, F.; Bruns, M.; Whittaker, M. R.; Boyer, C.; Barker, P. J.; Davis, T. P.; Barner-Kowollik, C., A detailed surface analytical study of degradation processes in (meth)acrylic polymers. Journal of Polymer Science Part A: Polymer Chemistry 2012, 50 (9), 1801-1811. 
150. Konaganti, V. K.; Madras, G., Photooxidative and pyrolytic degradation of methyl methacrylate-alkyl acrylate copolymers. Polymer Degradation and Stability 2009, 94 (9), 1325-1335.

151. Bennet, F.; Lovestead, T. M.; Barker, P. J.; Davis, T. P.; Stenzel, M. H.; BarnerKowollik, C., Degradation of Poly(methyl methacrylate) Model Compounds at Constant Elevated Temperature Studied via High Resolution Electrospray Ionization Mass Spectrometry (ESI-MS). Macromolecular Rapid Communications 2007, 28 (16), 1593-1600.

152. Jackson, A. T.; Slade, S. E.; Scrivens, J. H., Characterisation of poly(alkyl methacrylate)s by means of electrospray ionisation-tandem mass spectrometry (ESI-MS/MS). International Journal of Mass Spectrometry 2004, 238 (3), 265-277.

153. Holland, B. J.; Hay, J. N., The kinetics and mechanisms of the thermal degradation of poly(methyl methacrylate) studied by thermal analysis-Fourier transform infrared spectroscopy. Polymer 2001, 42 (11), 4825-4835.

154. Song, J.; Fischer, C. H.; Schnabel, W., Thermal oxidative degradation of poly(methyl methacrylate). Polymer Degradation and Stability 1992, 36 (3), 261266.

155. Choi, J., Degradation of poly(methylmethacrylate) by deep ultraviolet, x-ray, electron beam, and proton beam irradiations. J. Vac. Sci. Technol. B 1988, 6 (6), 2286.

156. Fox, R. B.; Isaacs, L. G.; Stokes, S., Photolytic degradation of poly(methyl methacrylate). Journal of Polymer Science Part A: General Papers 1963, 1 (3), 10791086.

157. Lovell, P. A.; Sherratt, M. M.; Young, R. J., Mechanical Properties and Deformation Micromechanics of Rubber-Toughened Acrylic Polymers. In Toughened Plastics II, American Chemical Society: 1996; Chapter 15, pp 211-232.

158. Hosbein, K. N.; Swartz, N. A.; Clare, T. L., Electrochemical Identification and Categorization of the Protective Quality of Intact and Damaged Coatings. Electroanalysis 2014, n/a-n/a.

159. Czech, Z.; Agnieszka, K.; Ragańska, P.; Antosik, A., Thermal stability and degradation of selected poly(alkyl methacrylates) used in the polymer industry. J. Therm. Anal. Calorim. 2015, 119 (2), 1157-1161.

160. Jubete, E.; Liauw, C. M.; Jacobson, K.; Allen, N. S., Degradation of carboxylated styrene butadiene rubber based water born paints. Part 1: Effect of talc filler and titania pigment on UV stability. Polymer Degradation and Stability 2007, 92 (8), 1611-1621.

161. Brendley, W. H., Fundamentals of Acrylics: Acrylic Polymers. Paint and Varnish Production 1973, pp 19-72.

162. Allen, N. S.; Regan, C. J.; Mclntyre, R.; Johnson, B. W.; Dunk, W. A. E., The photooxidative degradation and stabilisation of water-borne acrylic coatings systems. Macromolecular Symposia 1997, 115, 1-26.

163. Fernández-Garća, M.; Fuente, J. L. d. I.; Cerrada, M. a. L.; Madruga, E. L., Preparation of poly(tert-butyl acrylate-g-styrene) as precursors of amphiphilic 
graft copolymers. 1. Kinetic study and thermal properties. Polymer 2002, 43 (11), 3173-3179.

164. Liang, R. H.; Tsay, F. D.; Gupta, A., Photodegradation of poly(n-butyl acrylate). Photochemical processes in polymeric systems. 8. Macromolecules 1982, 15 (4), 974-980.

165. Carniti, P.; Beltrame, P. L.; Armada, M.; Gervasini, A.; Audisio, G., Polystyrene thermodegradation. 2. Kinetics of formation of volatile products. Industrial \& Engineering Chemistry Research 1991, 30 (7), 1624-1629.

166. Wood, K. A., How can we effectively use accelerated methods to predict the decorative properties of PVDF-based coatings? - A practical approach. Progress in Organic Coatings 2014, (0).

167. Zhou, H.; Wang, H.; Niu, H.; Gestos, A.; Lin, T., Robust, Self-Healing Superamphiphobic Fabrics Prepared by Two-Step Coating of Fluoro-Containing Polymer, Fluoroalkyl Silane, and Modified Silica Nanoparticles. Advanced Functional Materials 2013, 23 (13), 1664-1670.

168. Herrera, N. N.; Putaux, J.-L.; David, L.; De Haas, F.; Bourgeat-Lami, E., Polymer/Laponite composite latexes: Particle morphology, film microstructure, and properties. Macromolecular Rapid Communications 2007, 28 (15), 1567-1573.

169. Wheeler, P. A.; Wang, J.; Mathias, L. J., Poly(methyl methacrylate)/laponite nanocomposites: Exploring covalent and ionic clay modifications. Chemistry of Materials 2006, 18 (17), 3937-3945.

170. Tawari, S. L.; Koch, D. L.; Cohen, C., Electrical double-layer effects on the Brownian diffusivity and aggregation rate of Laponite clay particles. Journal of Colloid and Interface Science 2001, 240 (1), 54-66.

171. Herrera, N. N.; Letoffe, J. M.; Reymond, J. P.; Bourgeat-Lami, E., Silylation of laponite clay particles with monofunctional and trifunctional vinyl alkoxysilanes. Journal of Materials Chemistry 2005, 15 (8), 863-871.

172. Herrera, N. N.; Letoffe, J.-M.; Putaux, J.-L.; David, L.; Bourgeat-Lami, E., Aqueous Dispersions of Silane-Functionalized Laponite Clay Platelets. A First Step toward the Elaboration of Water-Based Polymer/Clay Nanocomposites. Langmuir 2004, 20 (5), 1564-1571.

173. Herrera, N. N.; Putaux, J.-L.; Bourgeat-Lami, E., Synthesis of polymer/Laponite nanocomposite latex particles via emulsion polymerization using silylated and cation-exchanged Laponite clay platelets. Progress in Solid State Chemistry 2006, $34(2-4), 121-137$.

174. Negrete-Herrera, N.; Putaux, J.-L.; David, L.; Bourgeat-Lami, E., Polymer/Laponite Composite Colloids through Emulsion Polymerization: Influence of the Clay Modification Level on Particle Morphology. Macromolecules 2006, 39 (26), 91779184 .

175. Macdonald, D. D., Reflections on the history of electrochemical impedance spectroscopy. Electrochimica Acta 2006, 51 (8-9), 1376-1388. 
176. Vanwesting, E. P. M.; Ferrari, G. M.; Dewit, J. H. W., The Determination of Coating Performance with Impedance Measurements .2. Water-Uptake of Coatings. Corrosion Science 1994, 36 (6), 957-977.

177. Castela, A. S.; Simões, A. M., An impedance model for the estimation of water absorption in organic coatings. Part I: A linear dielectric mixture equation. Corrosion Science 2003, 45 (8), 1631-1646.

178. Liu, B.; Li, Y.; Lin, H.; Cao, C. N., Electrochemical impedance spectroscopy study on the diffusion behavior of water through epoxy coatings. Corrosion 2003, 59 (9), 817820.

179. Hu, J. M.; Zhang, J. T.; Zhang, J. Q.; Cao, C. N., A novel method for determination of diffusion coefficient of corrosive species in organic coatings by EIS. Journal of Materials Science 2004, 39 (14), 4475-4479.

180. Philippe, L. V. S.; Lyon, S. B.; Sammon, C.; Yarwood, J., Validation of electrochemical impedance measurements for water sorption into epoxy coatings using gravimetry and infra-red spectroscopy. Corrosion Science 2008, 50 (3), 887896.

181. Shashoua, $Y_{\text {.; }}$ Matthiesen, $H_{\text {., }}$ Protection of iron and steel in large outdoor industrial heritage objects. Corrosion Engineering Science and Technology 2010, 45 (5), 357-361.

182. Wheeler, P. A.; Wang, J.; Baker, J.; Mathias, L. J., Synthesis and Characterization of Covalently Functionalized Laponite Clay. Chemistry of Materials 2005, 17 (11), 30123018.

183. Taddei, P.; Balducci, F.; Simoni, R.; Monti, P., Raman, IR and thermal study of a new highly biocompatible phosphorylcholine-based contact lens. Journal of Molecular Structure 2005, 744-747 (0), 507-514.

184. Cousin, F.; Cabuil, V.; Grillo, I.; Levitz, P., Competition between Entropy and Electrostatic Interactions in a Binary Colloidal Mixture of Spheres and Platelets. Langmuir 2008, 24 (20), 11422-11430.

185. Fonseca, D. M.; MÃ@heust, Y.; Fossum, J. O.; Knudsen, K. D.; Parmar, K. P. S., Phase diagram of polydisperse Na-fluorohectoriteâ $€$ "water suspensions: A synchrotron small-angle x-ray scattering study. Physical Review E 2009, 79 (2), 021402.

186. Hinderliter, B. R.; Croll, S. G., Simulation of transient electrochemical impedance spectroscopy due to water uptake or oxide growth. Electrochimica Acta 2009, 54 (23), 5344-5352.

187. Kobayashi, S.; Nishikawa, T.; Takenobu, T.; Mori, S.; Shimoda, T.; Mitani, T.; Shimotani, H.; Yoshimoto, N.; Ogawa, S.; Iwasa, Y., Control of carrier density by self-assembled monolayers in organic field-effect transistors. Nat Mater 2004, 3 (5), 317-322.

188. Jung, H.; Kim, H.-M.; Choy, Y. B.; Hwang, S.-J.; Choy, J.-H., Laponite-based nanohybrid for enhanced solubility and controlled release of itraconazole. International Journal of Pharmaceutics 2008, 349 (1-2), 283-290. 
189. Bedoya, F. E.; Gallego, L. M.; Bermudez, A.; Castano, J. G.; Echeverria, F.; Calderon, J. A.; Maya, J. G., New strategy to assess the performance of organic coatings during ultraviolet condensation weathering tests. Electrochimica Acta 2014, 124, 119-127.

190. Katan, E.; Narkis, M.; Siegmann, A., The effect of some fluoropolymers' structures on their response to UV irradiation. Journal of Applied Polymer Science 1998, 70 (8), 1471-1481.

191. Kimura, H.; Sakurai, M.; Sugiyama, T.; Tsuchida, A.; Okubo, T.; Masuko, T., Dispersion state and rheology of hectorite particles in water over a broad range of salt and particle concentrations. Rheologica Acta 2011, 50 (2), 159-168.

192. Alexandre, M.; Dubois, P., Polymer-layered silicate nanocomposites: preparation, properties and uses of a new class of materials. Materials Science and Engineering: $R$ : Reports 2000, 28 (1-2), 1-63. 


\section{Appendix A: Physical properties of wax coatings: DSC and Gloss}

Table A1 Changes in gloss upon weathering

\begin{tabular}{|c|c|c|c|c|}
\hline Type of Aging & Time & $\begin{array}{l}\text { Gloss } 85^{\circ}(\% \mathrm{R}) \\
\text { initial }\end{array}$ & $\begin{array}{l}\text { Gloss } 85^{\circ}(\% \mathrm{R}) \\
\text { after weathering }\end{array}$ & $\Delta$ Gloss $(\% \mathrm{R})$ \\
\hline $\mathrm{B} / \mathrm{Br} / \mathrm{PDX}$ & $36 \mathrm{mo}$ & $33 \pm 5$ & $1.4 \pm 0.2$ & $-95.8 \pm 19.9$ \\
\hline $\mathrm{B} / \mathrm{BrP} / \mathrm{PDX}$ & $36 \mathrm{mo}$ & $39 \cdot 9 \pm 3 \cdot 5$ & $3 \cdot 9 \pm 1.9$ & $-90.2 \pm 45$ \\
\hline $\mathrm{B} / \mathrm{Br} / \mathrm{FL}$ & $18 \mathrm{mo}$ & $23 \cdot 5 \pm 3 \cdot 3$ & $4.7 \pm 0.6$ & $-80 \pm 15.2$ \\
\hline $\mathrm{B} / \mathrm{BrP} / \mathrm{FL}$ & $18 \mathrm{mo}$ & $36.3 \pm 4.6$ & $4.3 \pm 0.7$ & $-88.2 \pm 18.2$ \\
\hline $\mathrm{B} / \mathrm{Br} / \mathrm{QUV}$ & $500 \mathrm{~h}$ & $36.2 \pm 3.4$ & $9 \cdot 3 \pm 3 \cdot 3$ & $-74 \cdot 3 \pm 27 \cdot 3$ \\
\hline B/BrP/QUV & $500 \mathrm{~h}$ & $41.9 \pm 3.1$ & $12.1 \pm 3.0$ & $-71.1 \pm 18.4$ \\
\hline $\mathrm{R} / \mathrm{Br} / \mathrm{QUV}$ & $1250 \mathrm{~h}$ & $50.6 \pm 0.8$ & $41.9 \pm 1.2$ & $-17.2 \pm 0.56$ \\
\hline R/BrP/QUV & $1500 \mathrm{~h}$ & $53 \cdot 3 \pm 1.2$ & $43.9 \pm 6.3$ & $-17.6 \pm 2.6$ \\
\hline R/St/PDX & $36 \mathrm{mo}$ & $46.9 \pm 3 \cdot 5$ & $13.8 \pm 7.1$ & $-70.6 \pm 36.7$ \\
\hline R/StP/PDX & $36 \mathrm{mo}$ & $42.3 \pm 3 \cdot 4$ & $16 \pm 8.4$ & $-62.2 \pm 33$ \\
\hline $\mathrm{R} / \mathrm{St} / \mathrm{FL}$ & $6 \mathrm{mo}$ & $50.2 \pm 2.2$ & $\mathrm{n} / \mathrm{a}$ & $\mathrm{n} / \mathrm{a}$ \\
\hline $\mathrm{R} / \mathrm{StP} / \mathrm{FL}$ & $6 \mathrm{mo}$ & $38.6 \pm 4$ & $\mathrm{n} / \mathrm{a}$ & $\mathrm{n} / \mathrm{a}$ \\
\hline R/St/QUV & $1250 \mathrm{~h}$ & $41.7 \pm 3.4$ & $26.5 \pm 2.9$ & $-36.5 \pm 4.97$ \\
\hline R/StP/QUV & $1500 \mathrm{~h}$ & $35.2 \pm 3.5$ & $46.4 \pm 3.8$ & $31.8 \pm 4.10$ \\
\hline
\end{tabular}

$\mathrm{R}=$ Renaissance wax, $\mathrm{B}=$ Butcher's wax, $\mathrm{Br}=$ bronze, $\mathrm{St}=$ steel, $\mathrm{P}=$ patinated, $\mathrm{QUV}=$ accelerated UV-B, PDX = Portland, OR, and FL = South Florida
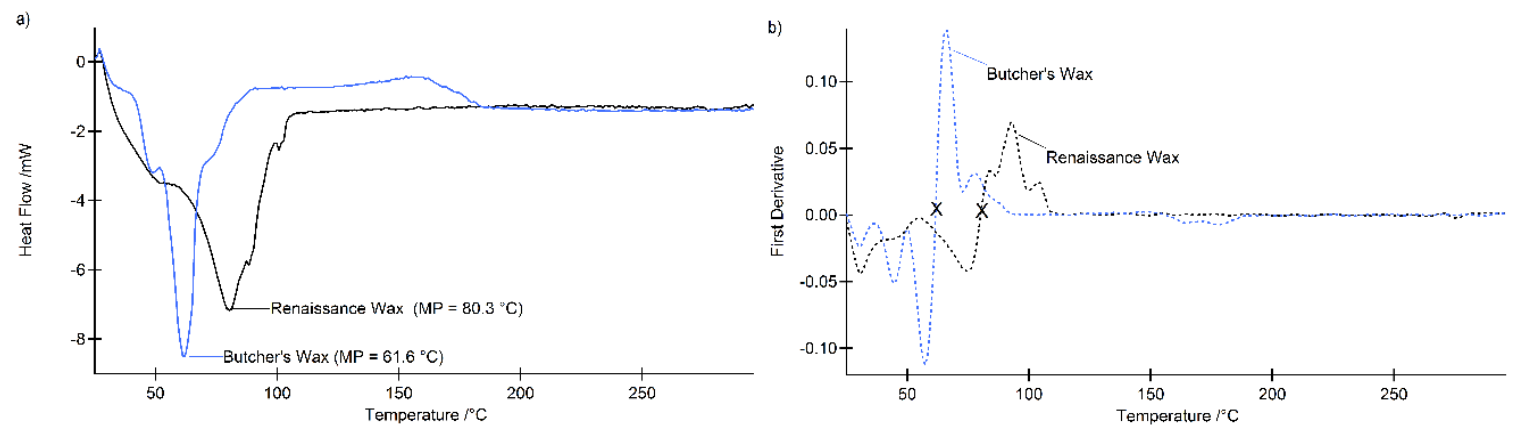

Figure A1. Differential scanning calorimetry thermograms of a) heat flow and b) the first derivative obtained in the determination of melting points for each wax mixture. The point at which the first derivative crossed zero was used as the overall melting point: $61.6^{\circ} \mathrm{C}$ for Butcher's wax and $80.3^{\circ} \mathrm{C}$ for Renaissance wax. 


\section{Appendix B: Micrographs and photographs of weathered wax samples}

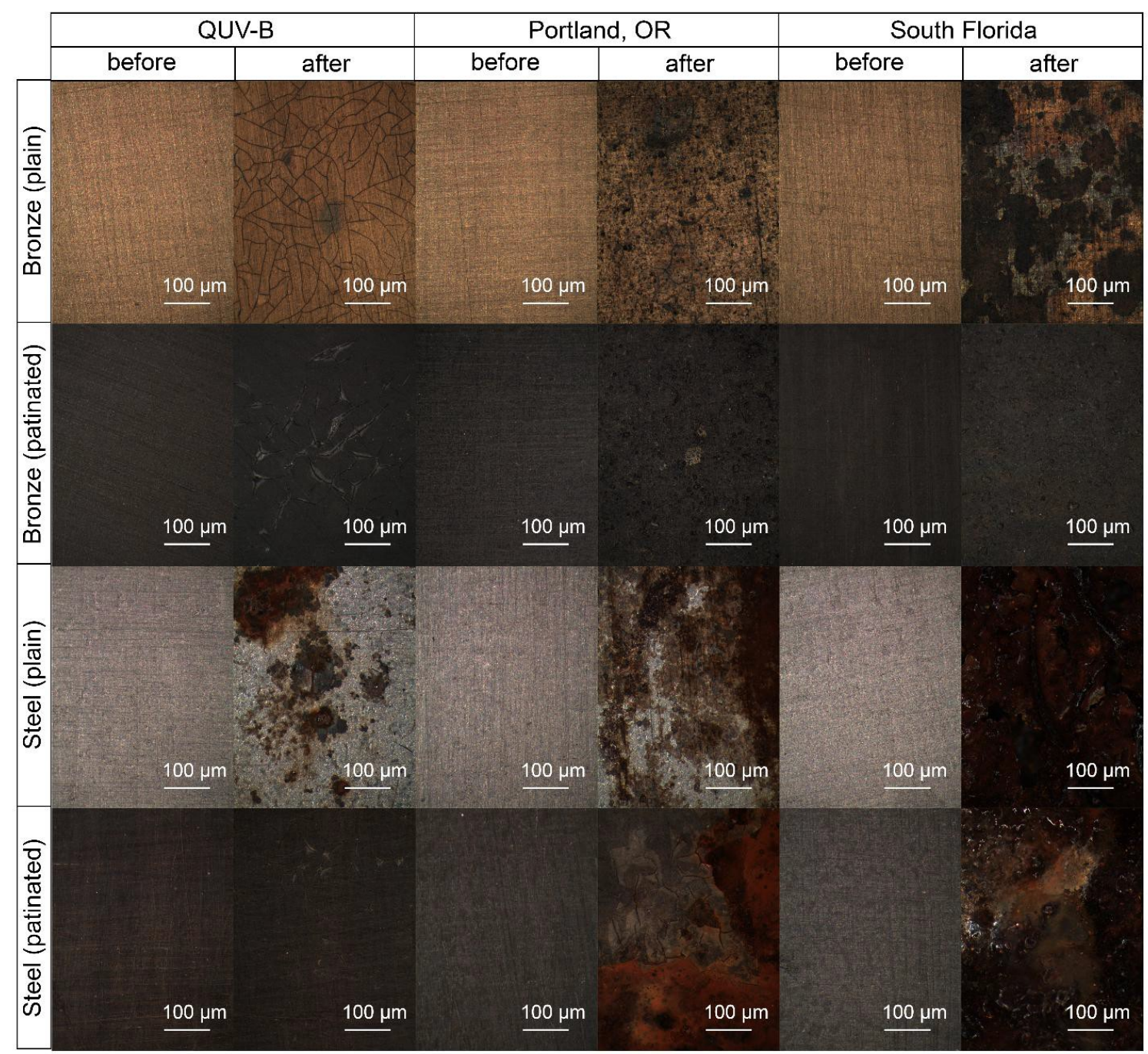

Figure B1. Micrographs of bronze and steel panels before and after weathering. Exposure times were 1500 hours for QUV-B, 18 months for Florida, and 36 months for Portland, Oregon. 

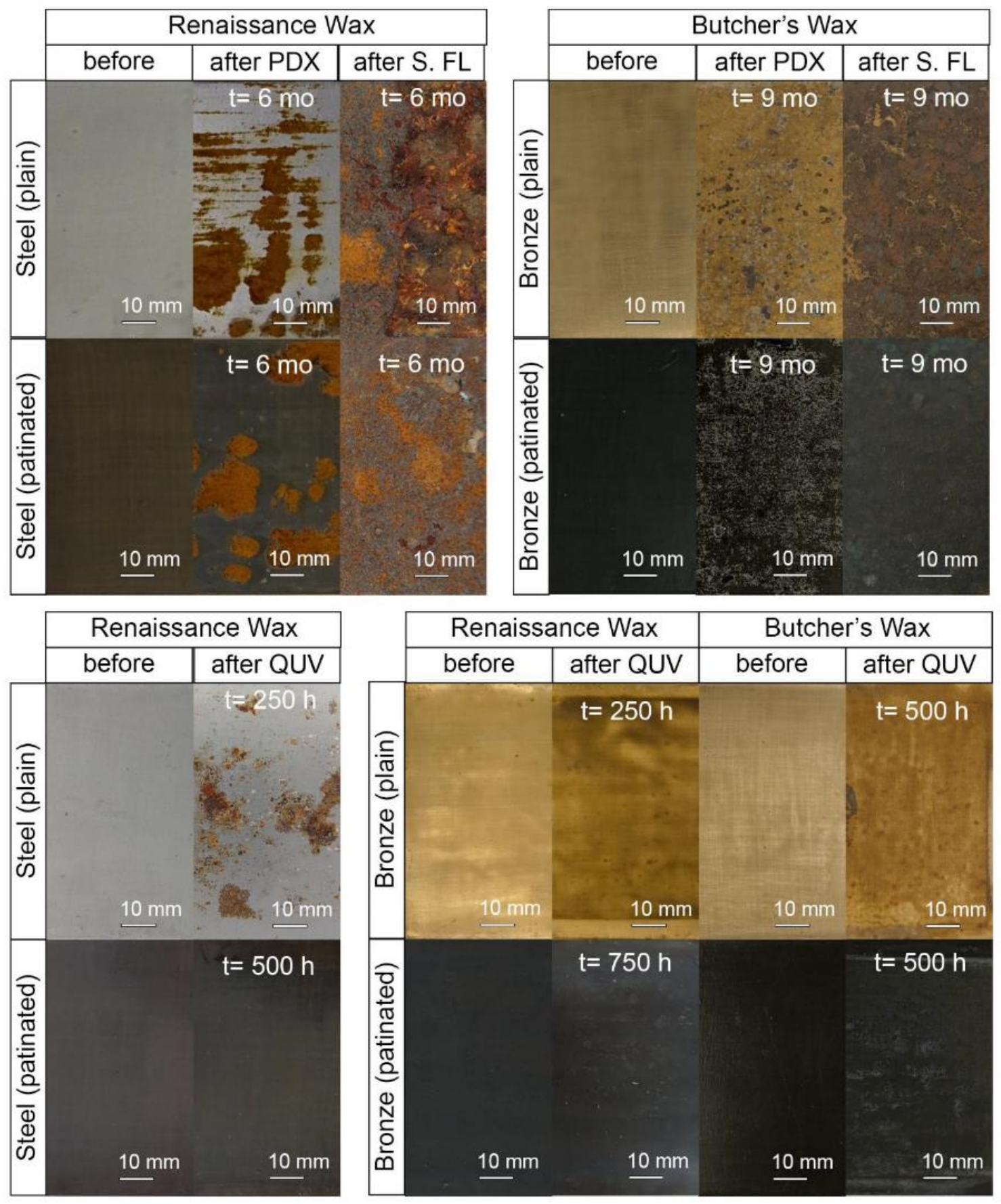

Figure B2. Photographs of waxed steel and bronze panels before and after outdoor weathering in Portland, OR (PDX), south Florida (S. FL) and accelerated weathering by QUV-B. Circuit values from EECs for these time points are marked " $\mathrm{X}$ " in Figure 2.7. 


\section{Appendix C: Corrosion products by accelerated and outdoor weathering}

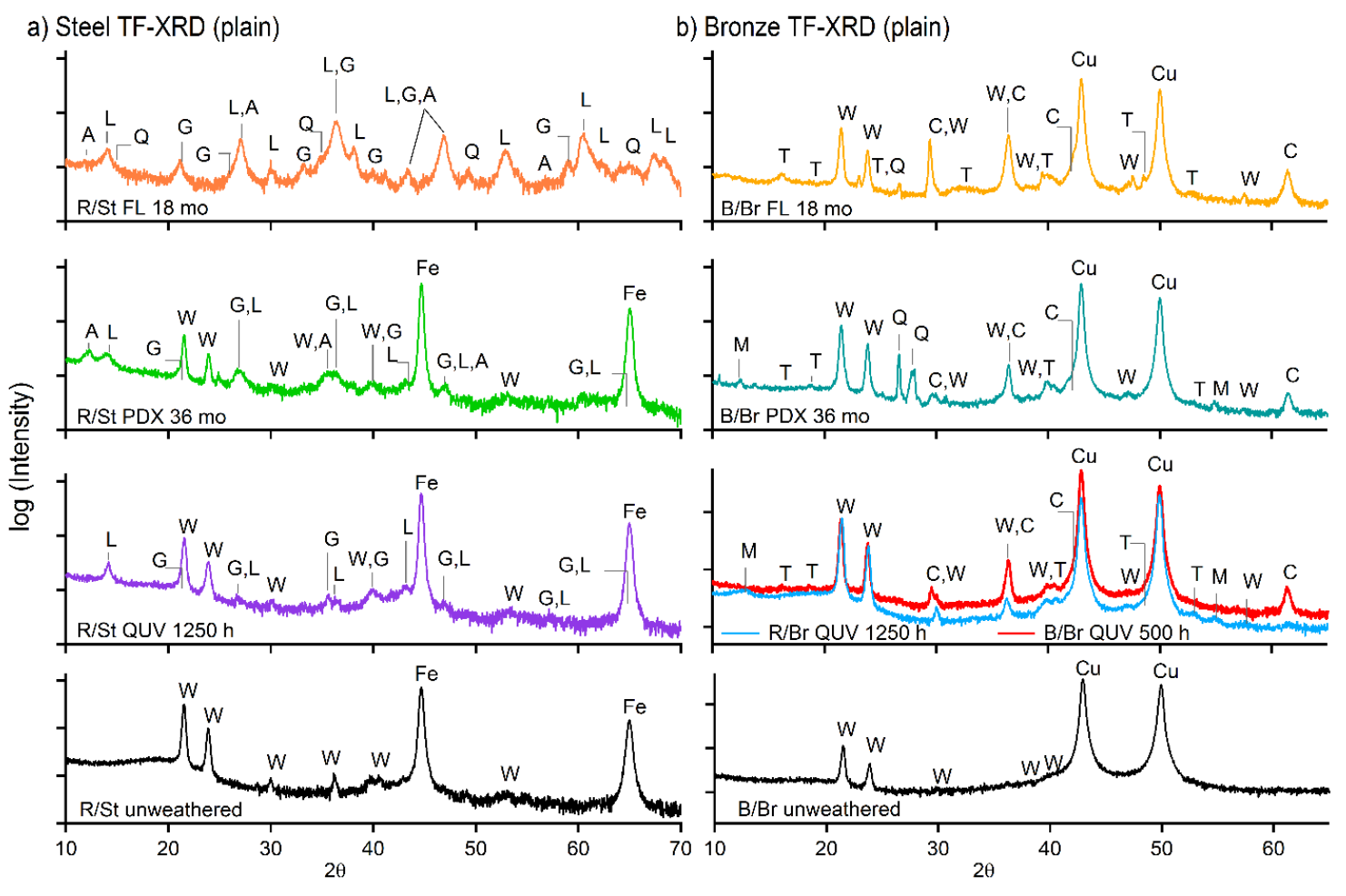

Figure $\mathrm{C}_{1}$. Identification of corrosion products by TF-XRD after various types of weathering. Diffraction patterns from a) plain steel and b) plain bronze. Sample type and substrate are noted by $\mathrm{Br}=$ bronze, $\mathrm{St}=$ steel, $\mathrm{B}=$ Butcher's Wax, $\mathrm{R}=$ Renaissance wax. Corrosion products notated by: $\mathrm{Cu}=$ Copper (JCPDS oo004-0836), $\mathrm{Fe}=$ Iron (JCDPS 00-006-0696), W = Wax (JCPDS 00-003-254), G = Goethite (JCPDS 00-0290713), A = Akaganite (JCPDS 00-013-0157), L= Lepidocrocite (JCPDS 01070-8045), Q = Layered silicate (JCPDS 00-002-0047), C = Cuprite (JCPDS 00-900-7497), $\mathrm{M}=$ Malachite (JCPDS 01-079-7849), and $\mathrm{T}=$ Atacamite (JCPDS 01-078-0372).

Corrosion products were detected on all substrates after weathering by TF-XRD. The identifications of each corrosion product and the other diffractions peaks due to either the substrate or the crystalline domains in the wax are labeled in Figure $C_{1}$ for plain and Figure $C_{2}$ for patinated substrates. In addition to the corrosion products, most plates weathered outdoors had a layered silicate (likely from dust/dirt) embedded in the wax. Additional peaks observed on patinated samples were related to species present in the patinating solution included on steel: ammonium iron sulphate, greigeite and on bronze: tetramethylammonium trichlorocuprate and copper sulphate hydroxide. The 
unweathered spectra often lacked those mineral peaks or those mineral peaks were smaller, likely because those species were initially poorly crystalline.

a) Steel TF-XRD (patinated)
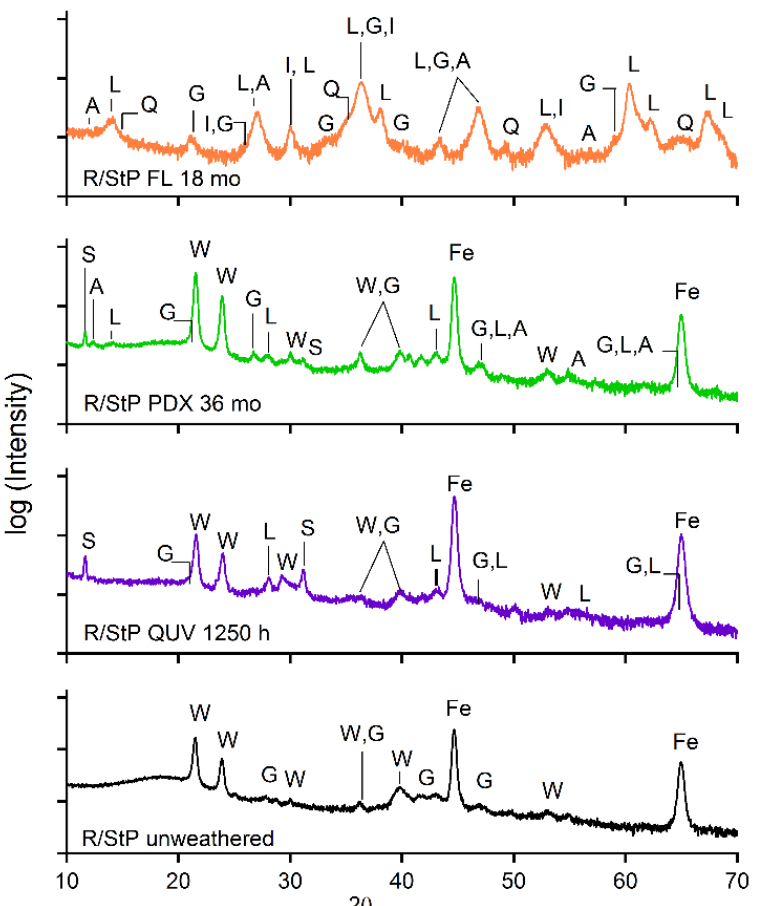

b) Bronze TF-XRD (patinated)
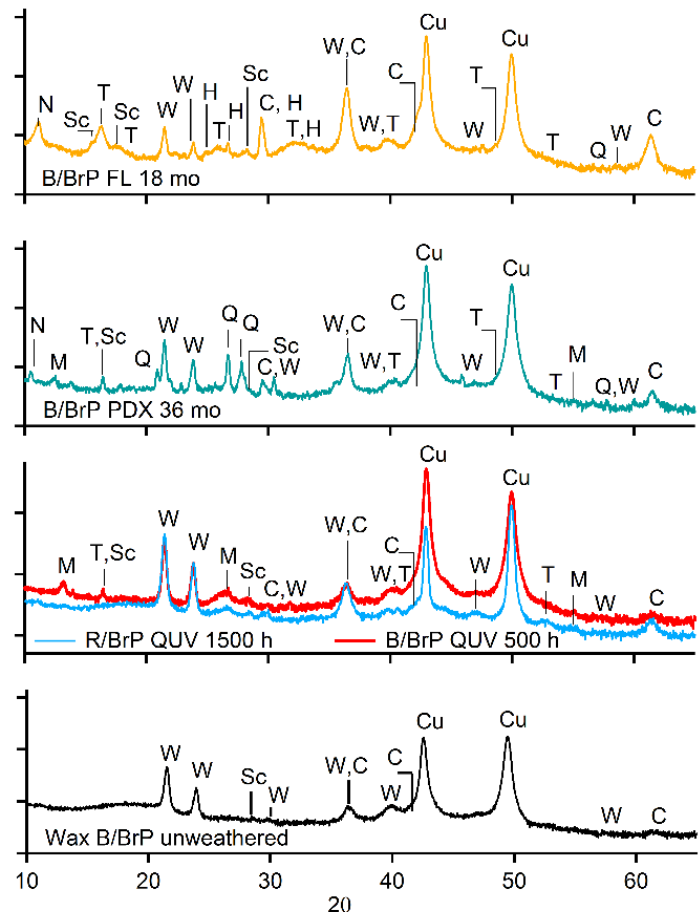

— B/BrP PDX 36 mo - B/BrP FL 18 mo
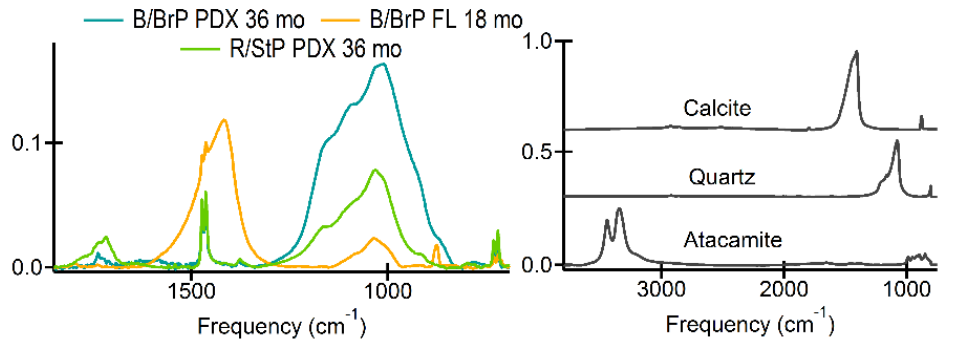

Figure C2. Corrosion products detected by TF-XRD and $\mu$-FT-IR of patinated samples after weathering. Diffraction patterns from a) patinated steel and b) patinated bronze and c) IR spectra of inorganics from both substrates versus reference spectra of calcite, quartz and atacamite. Sample type and substrate are noted by $\mathrm{Br}=$ bronze, $\mathrm{St}=$ steel, $\mathrm{B}=$ Butcher's Wax, $\mathrm{R}=$ Renaissance wax, $\mathrm{P}=$ patinated. Corrosion products are notated by: $\mathrm{Fe}=$ Iron (JCDPS oo- 006-0696), $\mathrm{Cu}=$ Copper (JCPDS 00-004-0836), W = Wax (JCPDS oo003-254), G = Goethite (JCPDS 00-029-0713), A = Akaganite (JCPDS 00-013-0157), L = Lepidocrocite (JCPDS 01070-8045), S = Ammonium Iron Sulfate (JCPDS 00-003-0043), I = Greigeite (JCPDS 01-0891998), Q = Layered silicate (JCPDS 00-001-1106), C =Cuprite (JCPDS o0-900-7497), T = Atacamite (JCPDS 01-078-0372), M = Malachite (JCPDS 01-079-7849), Sc = Copper sulfate hydroxide hydrate (JCPDS 00-0510321), and $\mathrm{N}=$ Tetramethylammonium trichlorcuprate (JCPDS 01-075-7162).

In general, the diffractograms of patinated substrates had sharper peaks than the plain substrates, indicating a greater crystallinity of those corrosion products. The chemical patina provides numerous sites for corrosion reactions to nucleate, which may 
explain the differences observed on the two substrate finishes. Some of the corrosion products were also detected by infrared spectroscopy, shown in Figure $\mathrm{C}_{2 \mathrm{C}}$, which suggested the presence of atacamite $\left[\mathrm{Cu}_{2}(\mathrm{OH})_{3} \mathrm{Cl}\right]$ and calcite $\left[\mathrm{CaCO}_{3}\right]$ on waxed bronze weathered in S. Florida from the following absorption peaks: hydroxyl stretching at 3447 and $3346 \mathrm{~cm}^{-1}$ (O-H stretch, atacamite), carbonic ester stretching near $1417 \mathrm{~cm}^{-1}$ (C-O stretch, calcite), carbonate bending at $876 \mathrm{~cm}^{-1}$ ( $\mathrm{C}-\mathrm{O}$ bend, calcite) and hydroxyl bending from $946-849 \mathrm{~cm}^{-1}$ (O-H deformation, atacamite). Atacamite was detected by XRD on every waxed bronze panel weathered outdoors. A quartz-like silicate was observed on both bronze and steel panels weathered in Portland, Oregon by the peaks at $1151 \mathrm{~cm}^{-1}$ and $1085 \mathrm{~cm}^{-1}$ (Si-O-Si, silicate) and $1030-1010 \mathrm{~cm}^{-1}$ (Si-O stretch, silicate). The hydroxyl bands present in Portland weathered steel were assigned to goethite where the high frequency band at $3620 \mathrm{~cm}^{-1}$ corresponds to hydroxyl stretching, and bending at $917 \mathrm{~cm}^{-1}$ (O-H deformation). 


\section{Appendix D: Detailed analysis of impedance spectra of wax coated substrates}

The first stage of the wax coatings' lifetime is the film at the start of the experiment (before weathering), here named Stage o, with representative spectra shown in Figure 2.3 b. Stage o data used in the creation of Figure 2.6 were fit with a two time-constant circuit model. The value of the coating capacitance, $C P E_{\text {coat, }}$ was at its lowest value throughout the entire weathering experiment in Stage o. A resistor corresponding to $R_{\text {pore }}$ was present, suggesting that small defects were present within the wax films. (Though scrupulous efforts were made to avoid producing defective films, these coatings were applied in a manner consistent with a conservators' application methods and having small defects are unavoidable.) The presence of defects within the film enabled the formation of a capacitive double-layer at the surface of the exposed metal and a resistive oxide layer (seen at this stage only when patinated substrates were used).

Stage 1a represents a well-annealed film. This stage was only observed on OUV weathered films, where average temperatures were higher than outdoor exposures. Relative to Stage o values, EIS spectral assignments to this stage included the following parameters: (1) R Rore $>100 \mathrm{~K} \Omega \cdot \mathrm{cm}^{2}$, (2) $T_{R C}<10 \mathrm{kHz}$, (3) $|Z|>150 \mathrm{M} \Omega \cdot \mathrm{cm}^{2}$. Circuit modeling showed that there exist two overlapping CPE elements (having values of $\mathrm{CPE}_{\text {coat- }-1}=0.16 \mathrm{nS} \cdot \mathrm{s}^{0.95}$ and $\mathrm{CPE}_{\text {coat } 2}=1.2 \mathrm{nS} \cdot \mathrm{s}^{0.75}$ ) that given their small value may correspond to two pathways through the wax. One path is more defective and waterlogged $\left(\mathrm{CPE}_{\mathrm{coat}-2}\right)$, possibly corresponding to a path through the inter-granular amorphous regions of the film and another corresponds to a pathway through the more crystalline regions. Renaissance waxed patinated bronze panels remained in Stage 1a until failure onset was observed at $1500 \mathrm{~h}$, moving it into stage $1 \mathrm{~b}$ based on the notable deviation in magnitude and phase plots from the earlier time points. Notably, the Renaissance waxed panels (both patinated and plain) showed the smallest EIS changes observed for any substrate under any weathering condition, and thus had the best protective qualities among all panels in this study. 
Stage $1 b$ was indicative of coating failure leading to the onset of corrosion. Both changes in capacitance (an increase) and resistance (a decrease) during weathering are consistent with a coating that holds more ions and increasingly allows the facile transport of ions through it as it ages. These combined effects lead to increased permittivity of the film and eventually cause corrosion. Film breakdown and corrosion growth were indicated by: (1) $100 \mathrm{k} \Omega \cdot \mathrm{cm}^{2}>R_{\text {pore }}>10 \mathrm{k} \Omega \cdot \mathrm{cm}^{2}$, (2) $10 \mathrm{kHz}<T_{R C}<100 \mathrm{kHz}$, and (3) $150 \mathrm{M} \Omega \cdot \mathrm{cm}^{2}>|\mathrm{Z}|>500 \mathrm{k} \Omega \cdot \mathrm{cm}^{2}$.

The accelerated (i) and outdoor (ii) weathered panels in stage 2 are shown in separate graphs for clarity. Loss of film thickness and corrosion growth were defined by: (1) $R_{\text {pore }}>10 \mathrm{k} \Omega \cdot \mathrm{cm}^{2}$, (2) $T_{R C}>100 \mathrm{KHz}$, (3) $R_{\text {ox }}>100 \Omega \cdot \mathrm{cm}^{2}$, and (4) $|\mathrm{Z}|<500 \mathrm{k} \Omega \cdot \mathrm{cm}^{2}$. All of the panels observed in Stage 2i were considered to have failed because of the presence of corrosion and/or film cracking and were removed from the study. The extent of damage in Stage zii was slightly more severe than zi because less wax remained on the panels weathered outdoors. Due to their prolonged progression through Stage 2ii, we concluded that plain samples weathered in Portland, Oregon had longer working lifetimes and better barrier properties at the same time point than did their patinated counterparts. Based on the more aggressive weathering conditions that the panels experienced in South Florida, the wax film supported by plain bronze also remained a better barrier since a larger film resistance $\left(R_{\text {pore }}\right)$ and smaller coating capacitance $\left(\mathrm{C}_{\text {coat }}\right)$ was observed at each time of measurement compared to its patinated counterpart.

Changes such as film delamination, ablation, and mechanical damage may decrease the protective nature of the entire coating to the extent that severe damage may be occurring across large areas of the metal surface. Stage 3 is defined as complete coating failure, where little to none of the original wax coating is present on the substrate and significant damage to the underlying metal substrate (e.g. corrosion and flaking) had occurred. An EIS spectrum from a failed coating is usually dominated by $R_{\text {bulk }}$ (the electrolyte solution resistance) over the majority of the frequency range (instead of $C_{\text {coat }}$ ) and by a few additional parameters: (1) $R_{\text {pore }}<500 \Omega \cdot \mathrm{cm}^{2},(2) T_{R C}<1$ 
$M H z$, (3) $R_{0 x}>100 \Omega \cdot \mathrm{cm}^{2}$, and (4) $|\mathrm{Z}|<500 \mathrm{k} \Omega \cdot \mathrm{cm}^{2}$. Some wax coated steel panels proceeded directly to Stage 3 (Stage 2 was not observed) by the first time point was measured because of the higher corrosion potential/faster rate of corrosion of steel. This indicated that wax coated steel, independent of patination, had completely failed and significant damage to the substrate occurred before 6 months of weathering exposure. 


\section{Appendix E: Impedance derivative analysis on steel and patinated bronze}

(a) B44 (patinated)

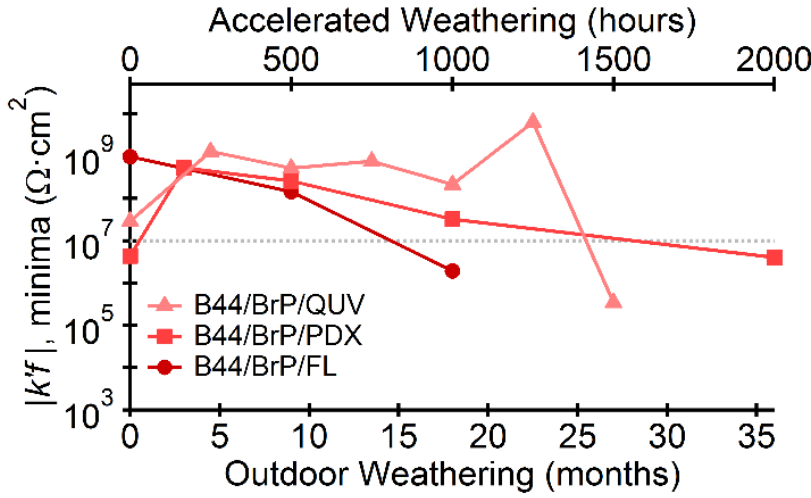

(b) Incralac (patinated)

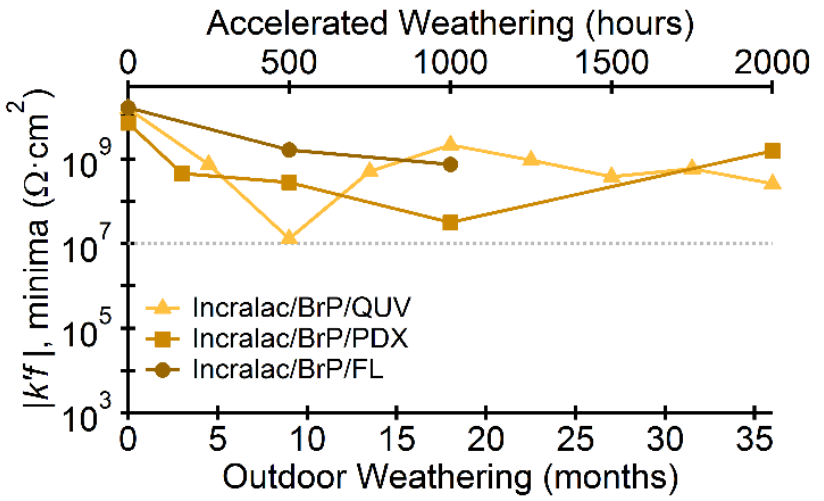

(c) WL81 (patinated)

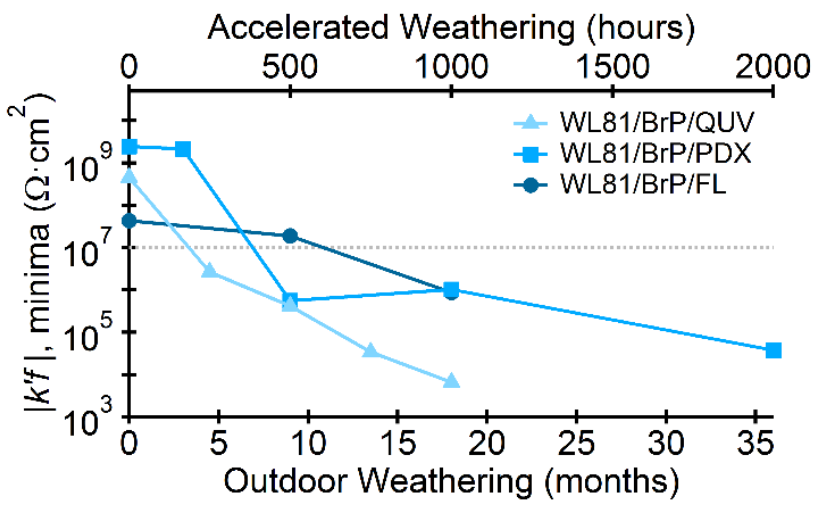

Figure E1. Derivative analysis of the impedance of patinated bronze panels coated with B44 (a), Incralac (b), and WL81 (c). 
(a) B44 (plain)

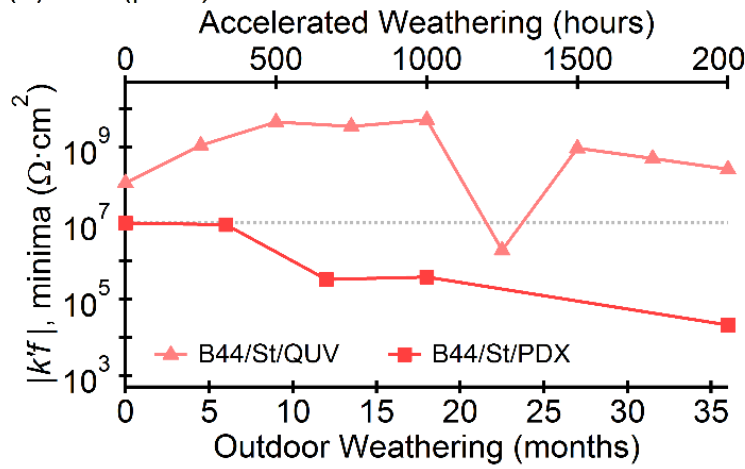

(c) Incralac (plain)

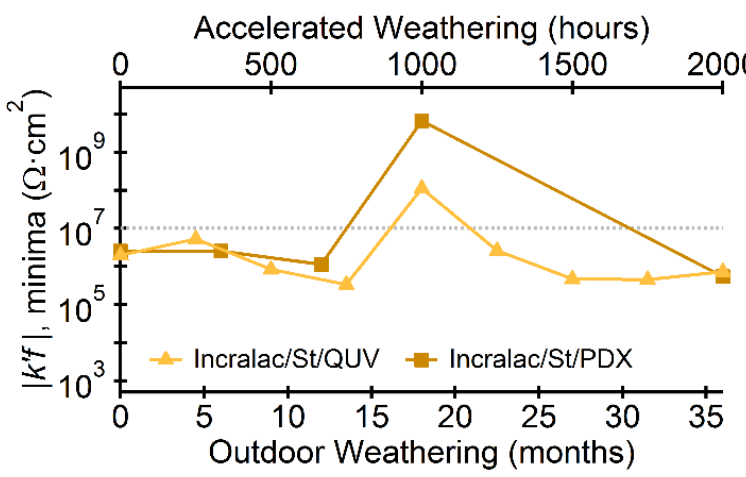

(e) WL81 (plain)

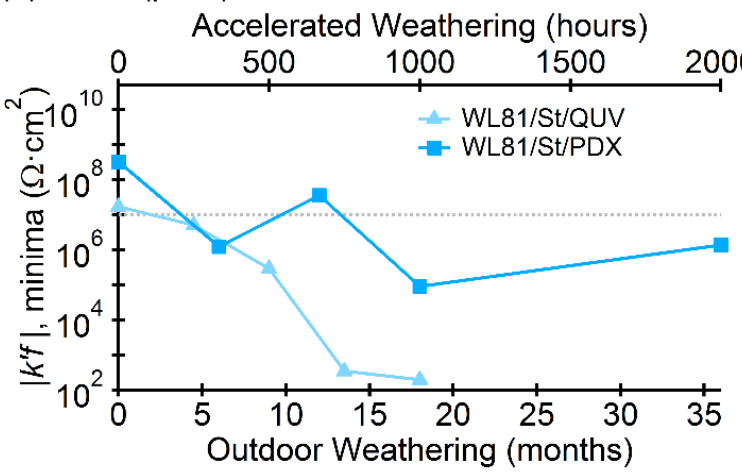

(b) B44 (patinated)

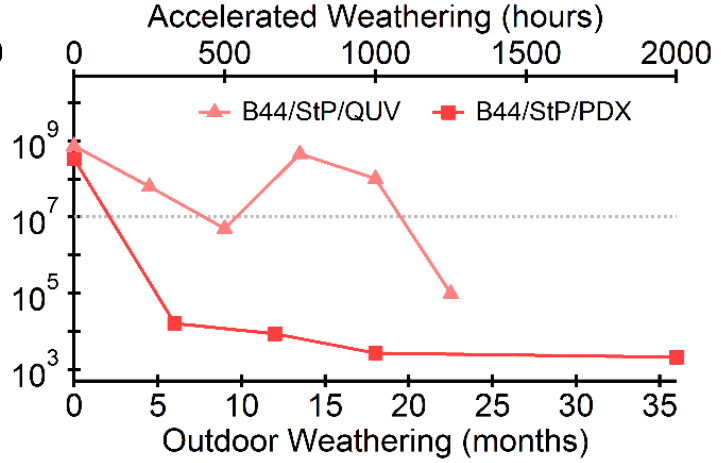

(d) Incralac (patinated)

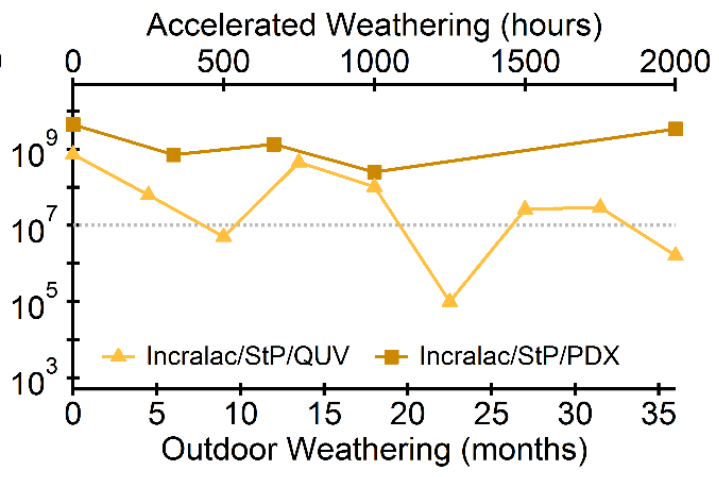

(f) WL81 (patinated)

Accelerated Weathering (hours)
$0 \quad 500 \quad 1000 \quad 1500 \quad 2000$

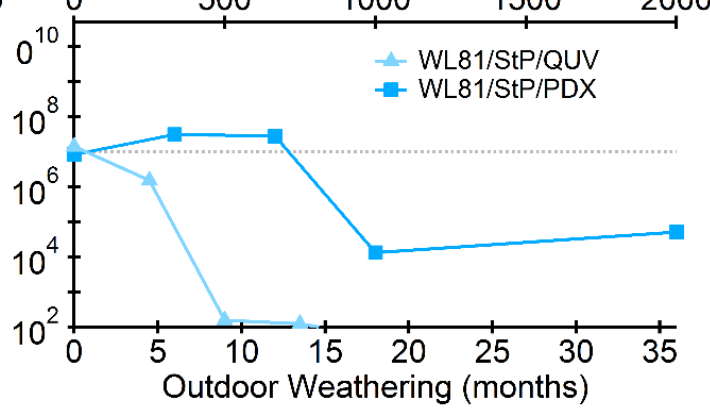

Figure E2. Derivative analysis of the impedance of plain and patinated steel panels coated with B44 (a-b), Incralac (c-d), and WL81 (e-f). 
(a) WL81 with additives (plain)

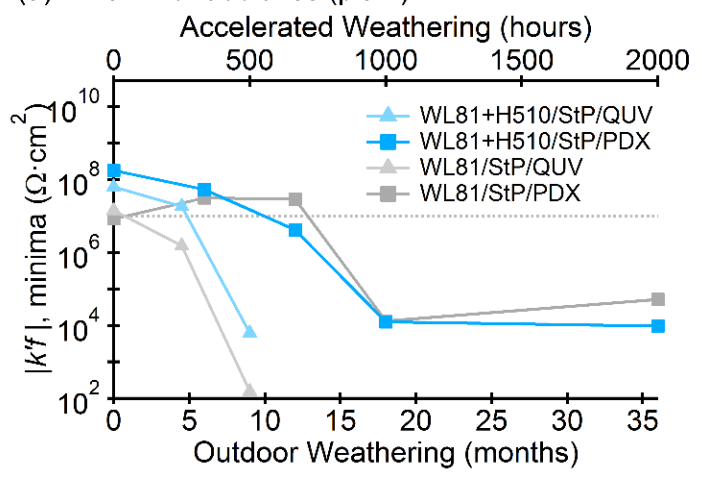

(b) WL81 with additives (patinated)

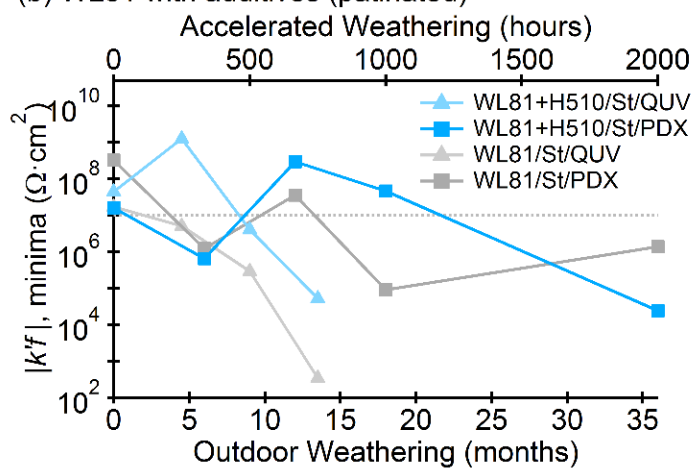

Figure E3. Derivative analysis of the impedance of plain (a) and patinated (b) steel panels coated with WL81 and Halox 510 added to the formulation. The coated panels with just WL81 are shown in gray for comparison.

(a) Kynar ARC (patinated)

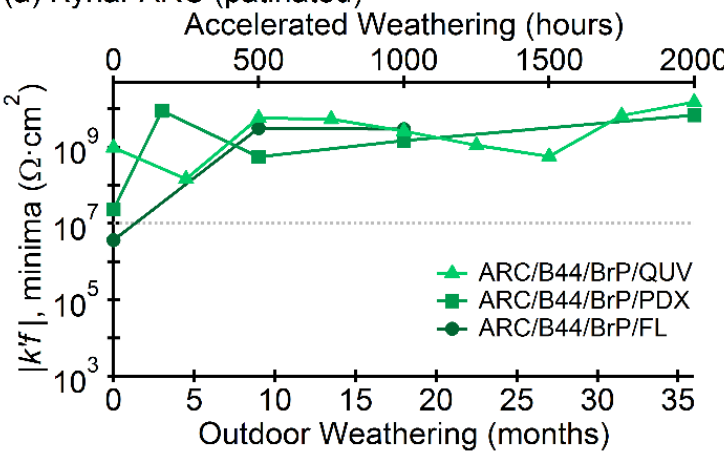

(b) Kynar FMA (patinated)

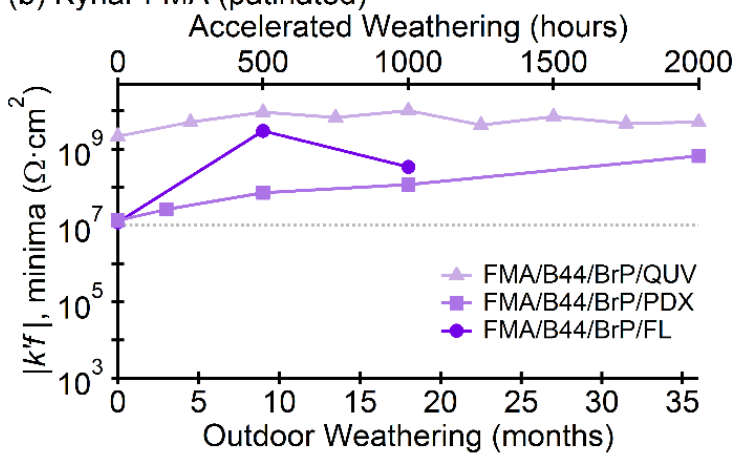

Figure E4. Quick Analysis of barrier properties of Kynar ARC (a) and FMA (b) with a B-44 primer on patinated bronze. 
(a) Kynar ARC (plain)

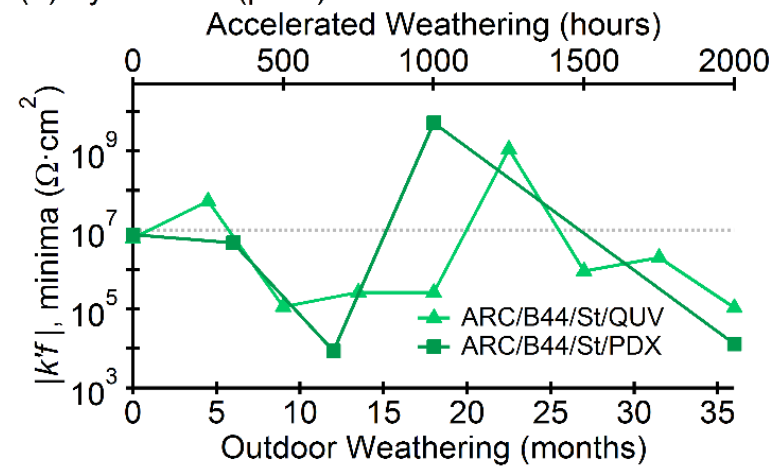

(b) Kynar ARC (patinated)

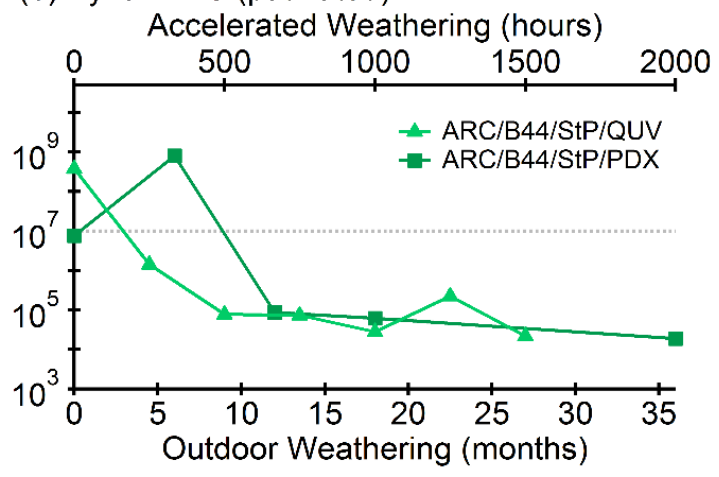

(c) Kynar FMA (plain)

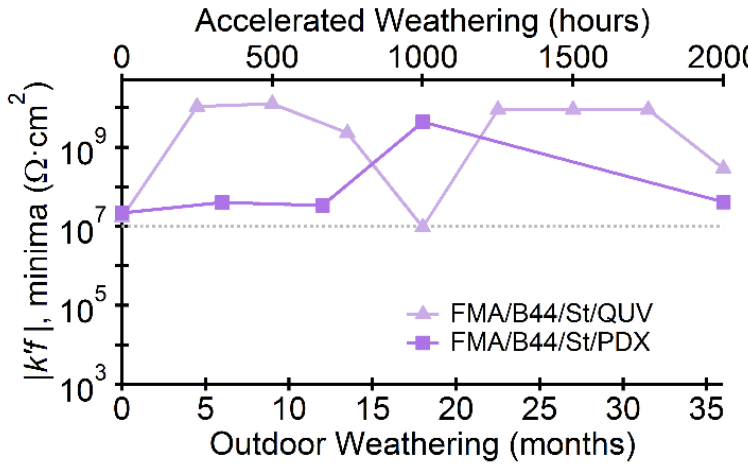

(d) Kynar FMA (patinated)

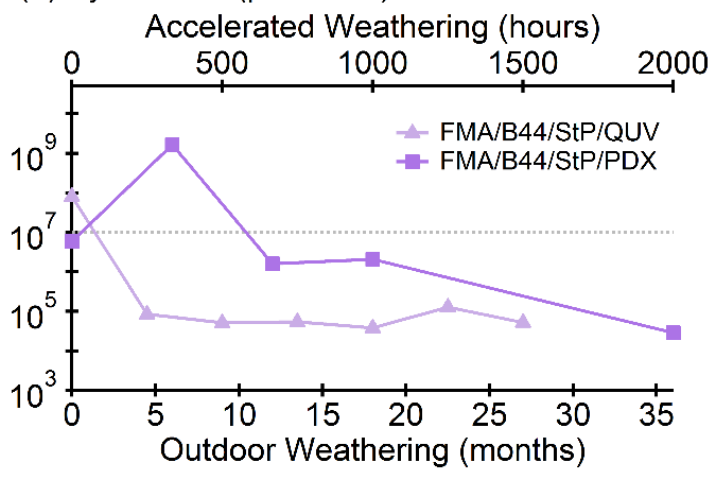

Figure E5. Quick Analysis of barrier properties of Kynar ARC and FMA with a B-44 primer on steel.

(a) Kynar ARC/B44+H630 (plain)

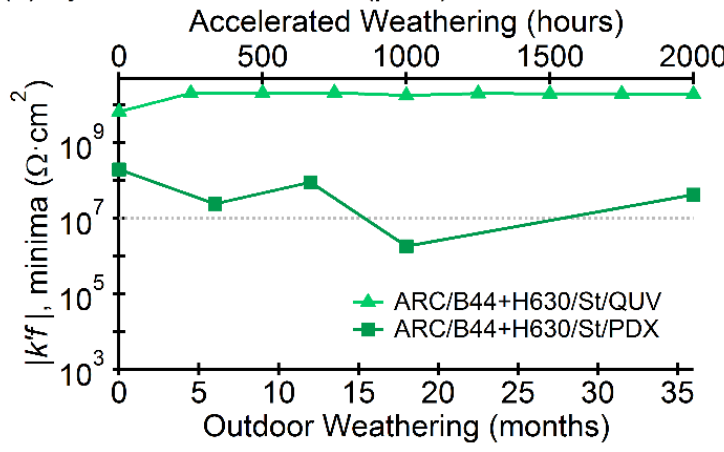

(b) Kynar ARC/B44+H630 (patinated)

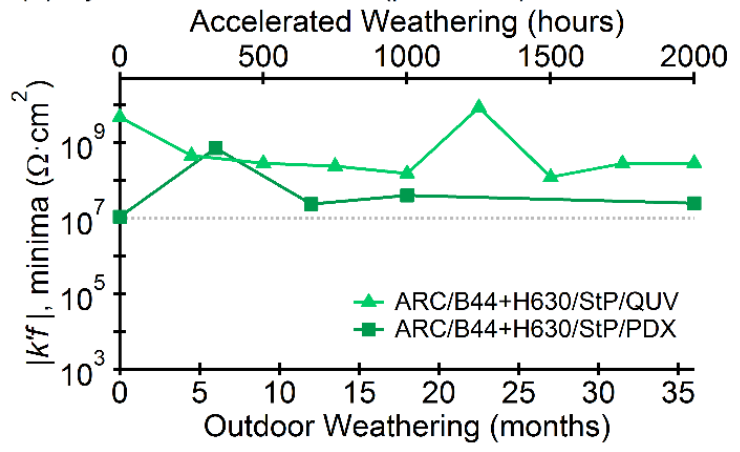

Figure E6. Analysis of barrier properties of a Kynar ARC top coat with a B44 base coat modified with Halox 630 on (a) plain and (b) patinated steel. 


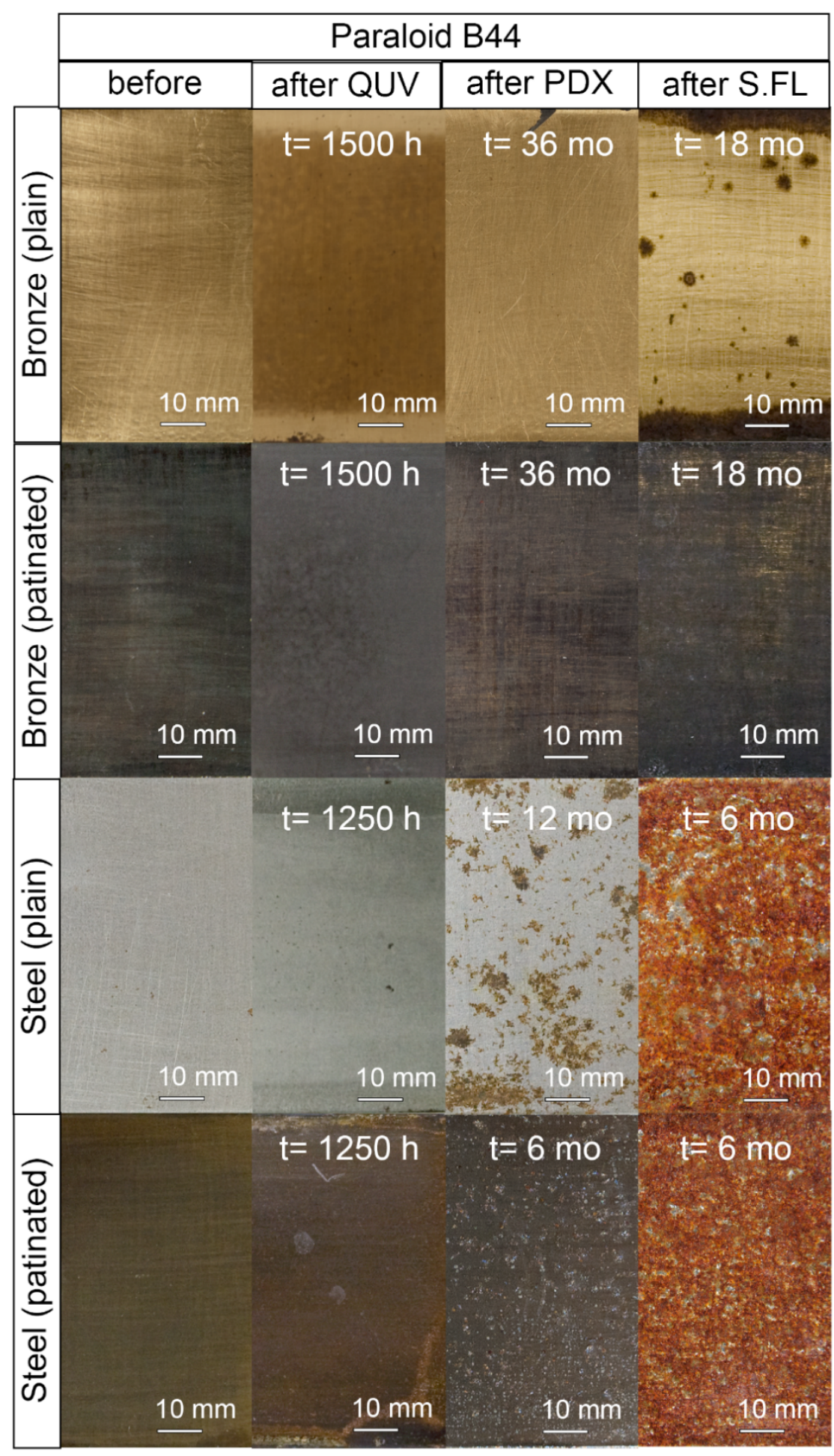

Figure E7. Photographs of B444 coated bronze and steel panels during weathering. The brightness was increased by $10 \%$ for photos of plain samples and $60-80 \%$ for samples with a patina. 


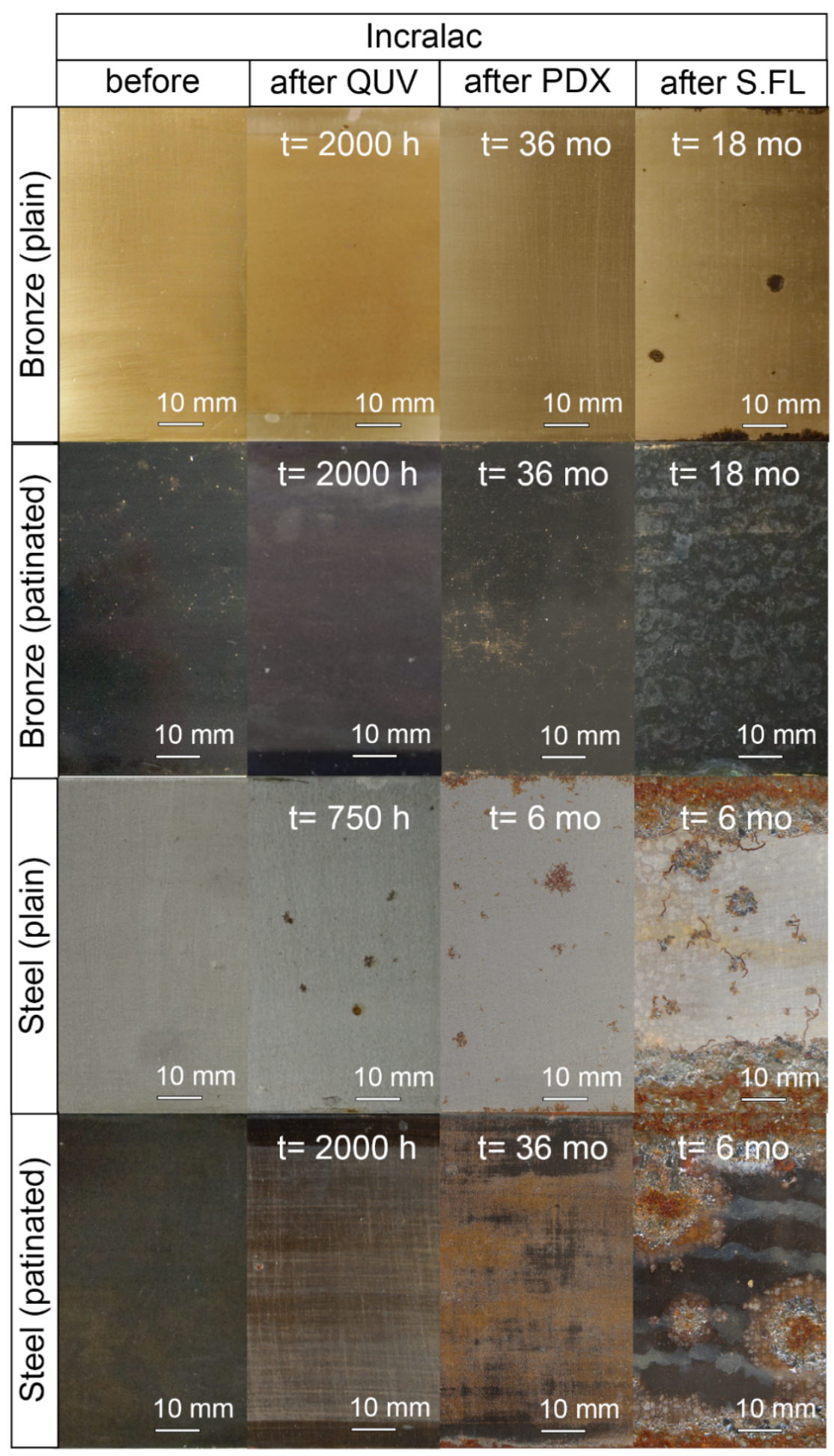

Figure E8. Photographs of Incralac coated bronze and steel panels during weathering. The brightness was increased by $10 \%$ for photos of plain samples and $60-80 \%$ for samples with a patina. 


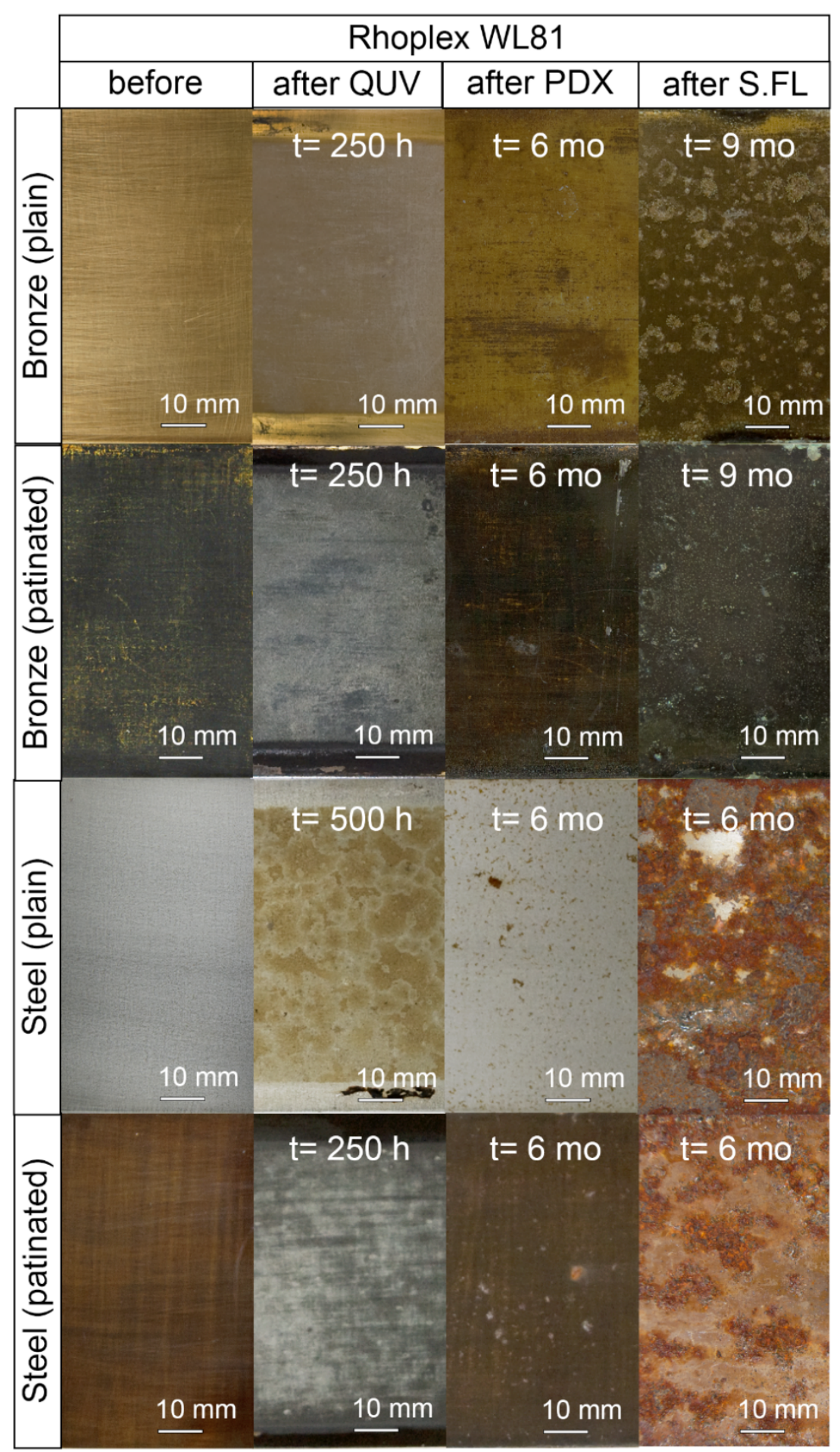

Figure E9. Photographs of WL81 coated bronze (with a BTA pretreatment) and steel (with Na Decanoate pretreatment) panels during weathering. The brightness was increased by $10 \%$ for photos of plain samples and $60-80 \%$ for samples with a patina. 


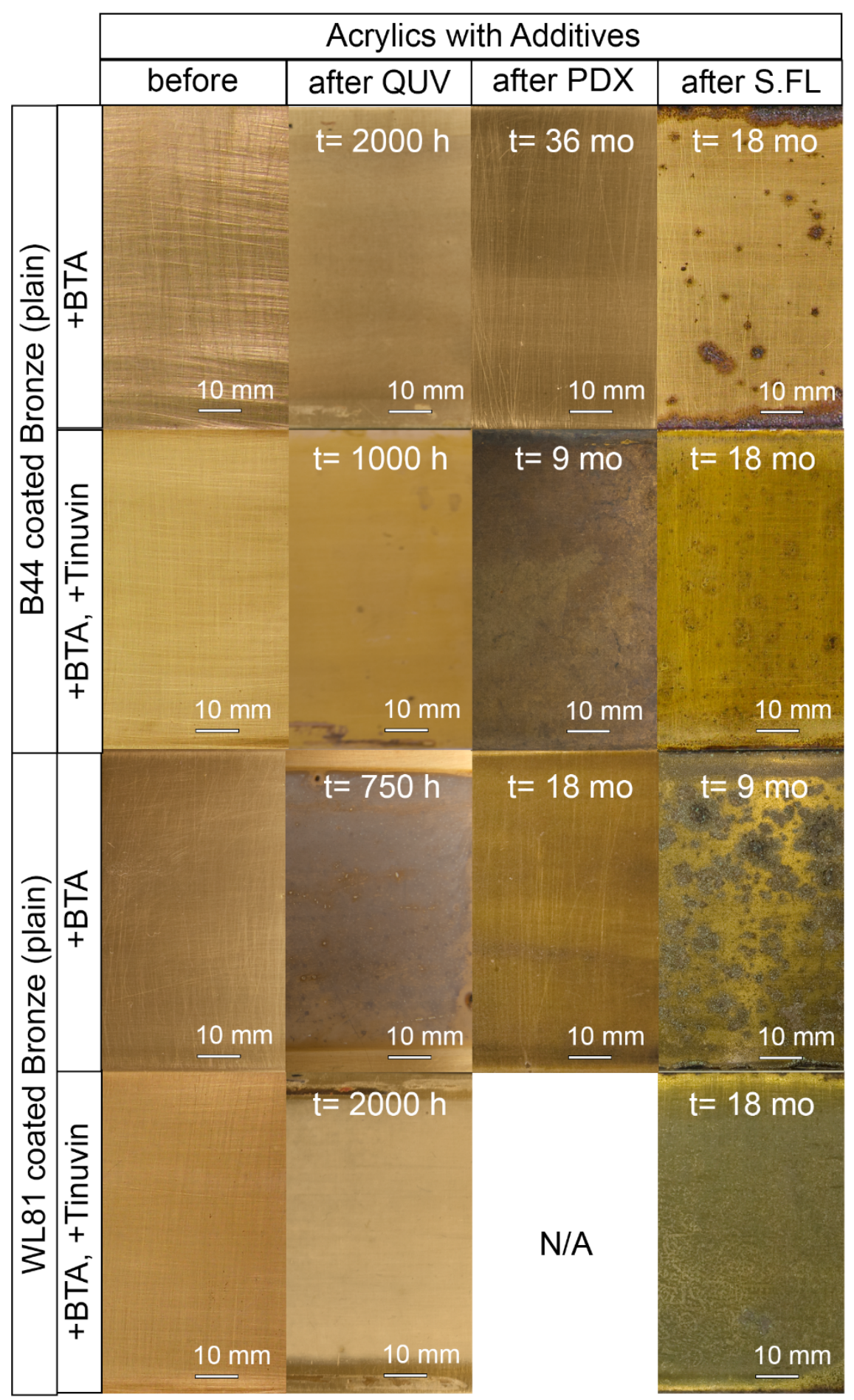

Figure E10. Photographs of coated bronze panels with B44 and WL81 (with BTA and Tinuvin added to both resins) during weathering. The brightness was increased by $10 \%$ for photos of plain samples. 


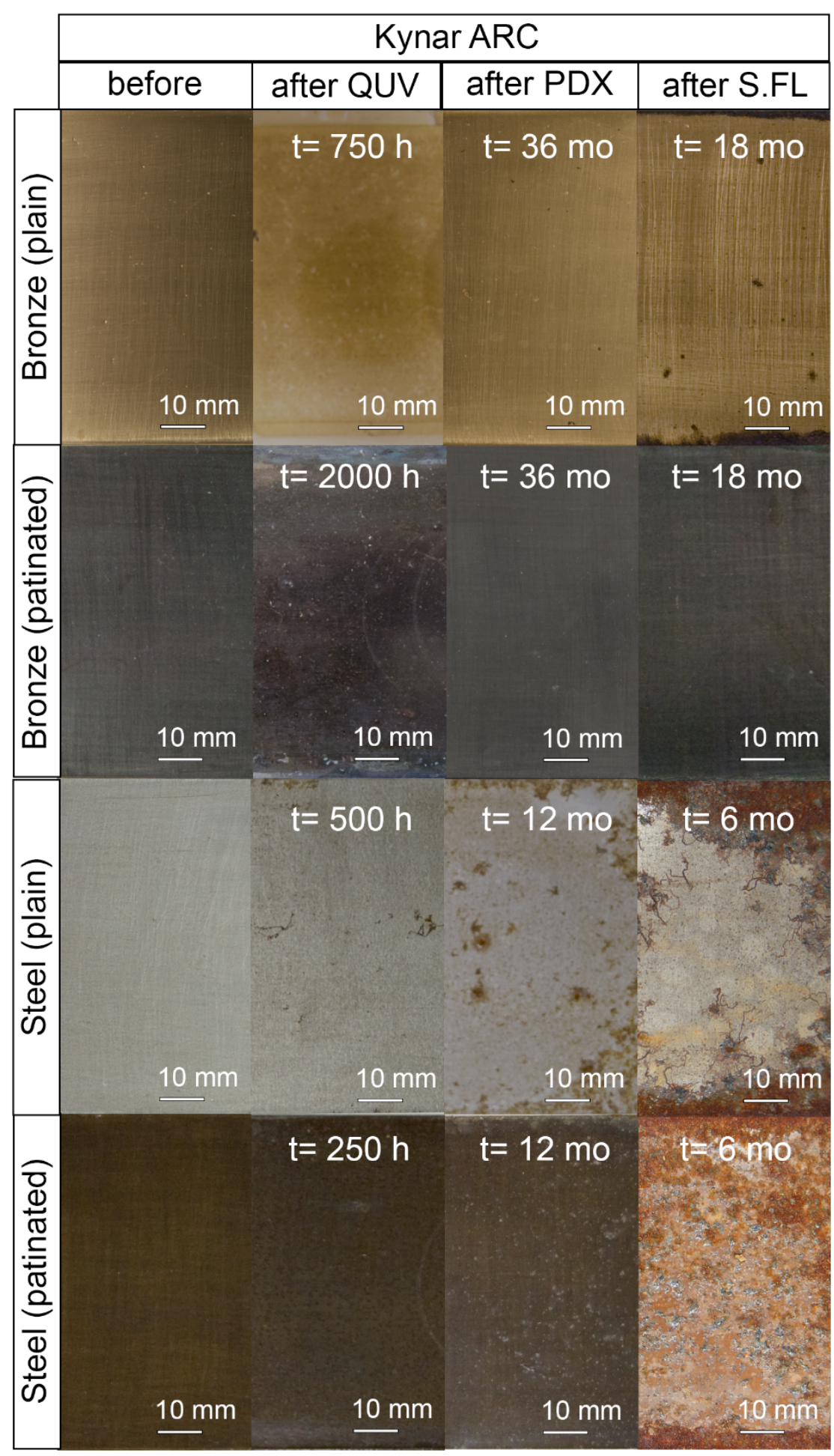

Figure E11. Photographs of ARC coated bronze and steel panels during weathering. The brightness was increased by $10 \%$ for photos of plain samples and $60-80 \%$ for samples with a patina. 


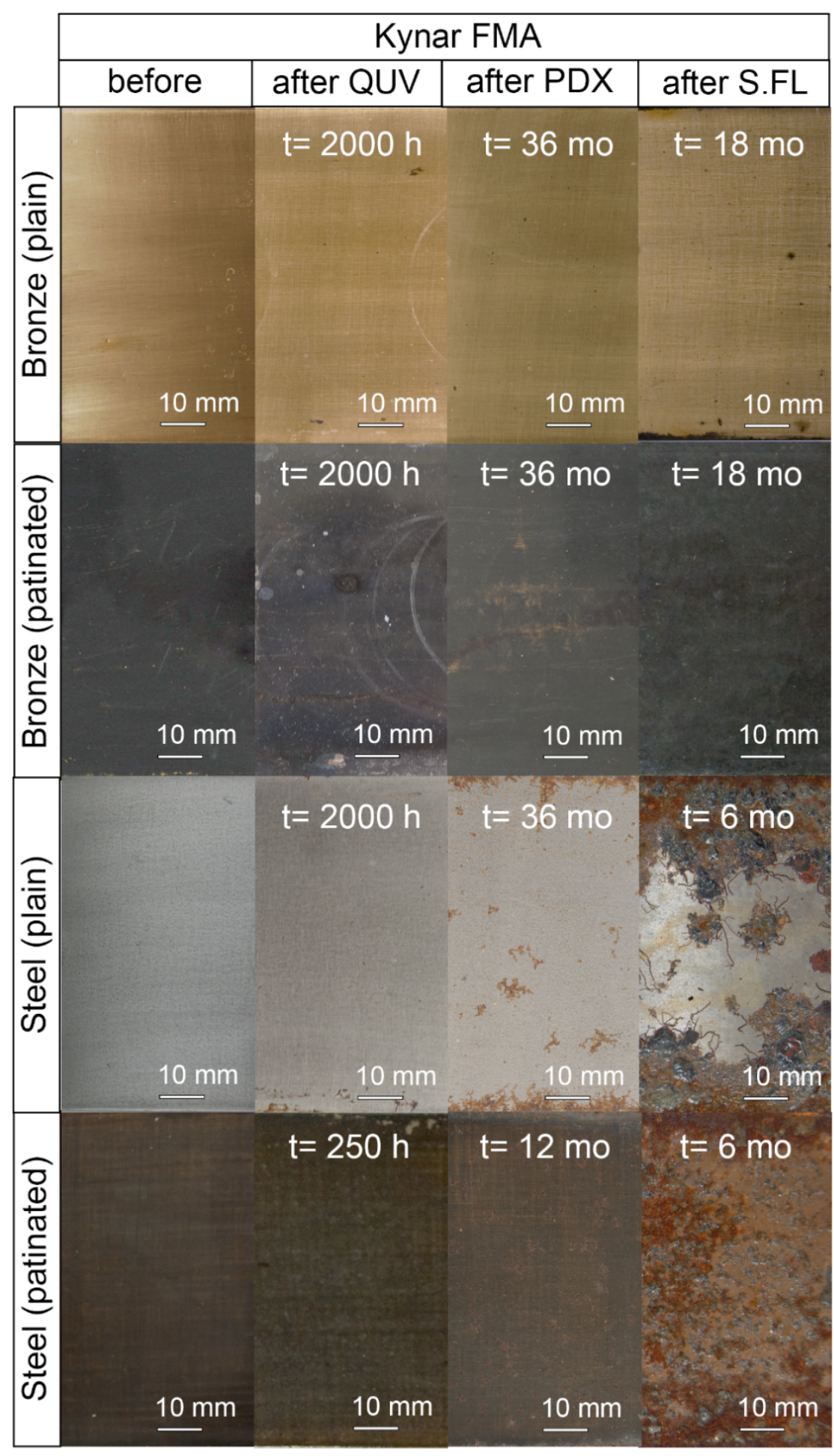

Figure E12. Photographs of FMA coated bronze and steel panels during weathering. The brightness was increased by $10 \%$ for photos of plain samples and $60-80 \%$ for samples with a patina. 


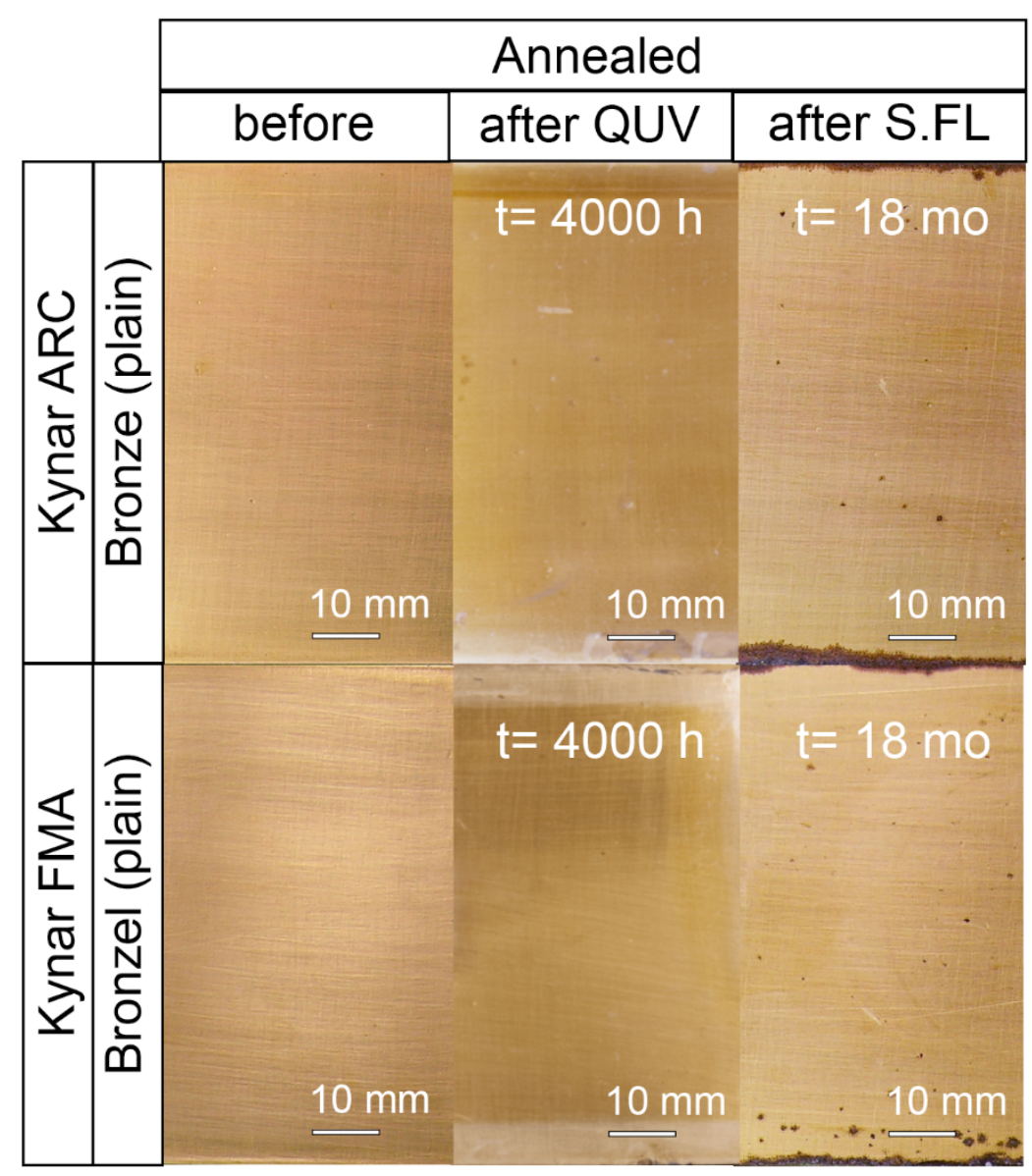

Figure E13. Photographs of annealed Kynar ARC and FMA coated bronze panels during weathering. The brightness was increased by $10 \%$ for photos of plain samples. 


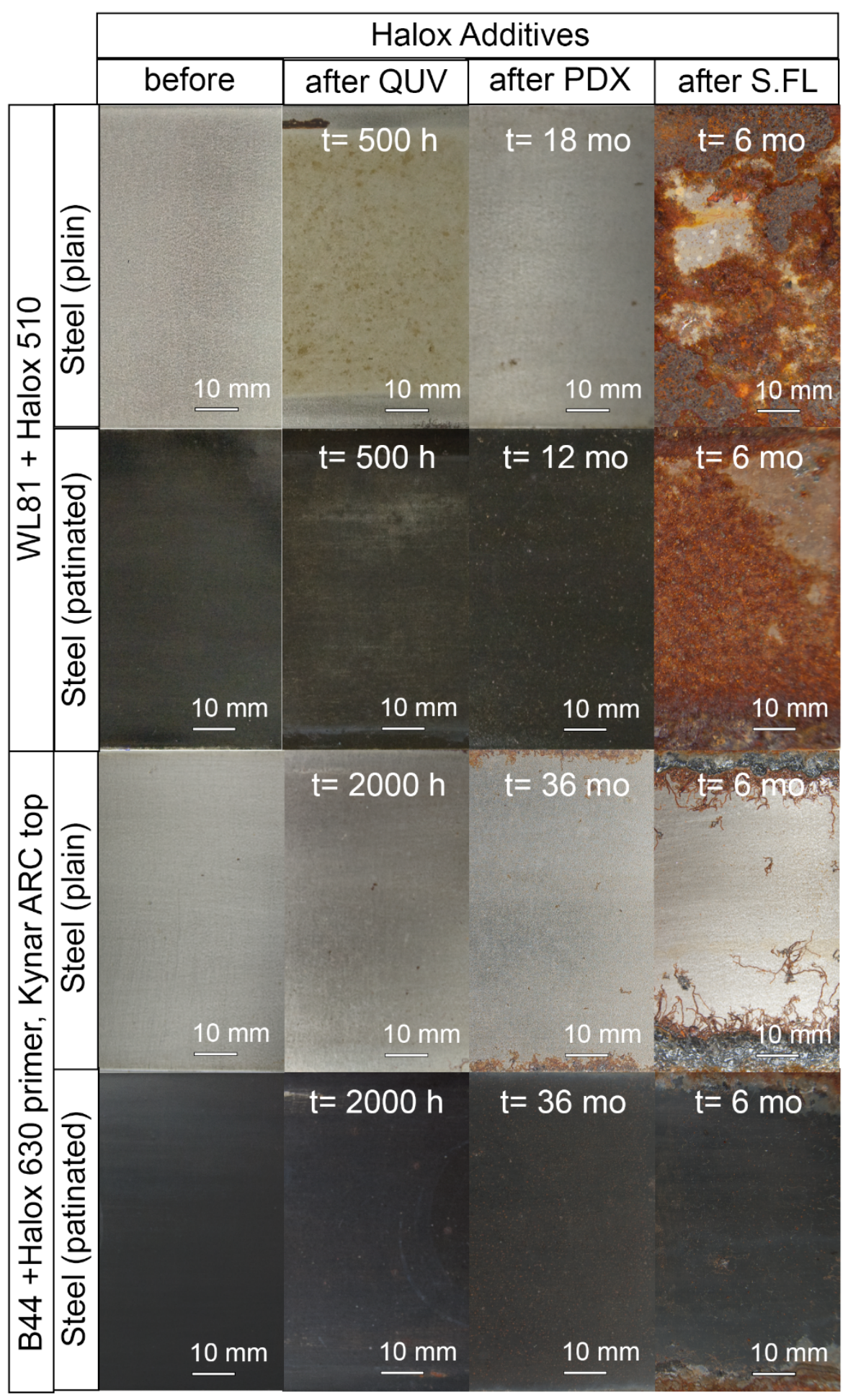

Figure E14. Photographs of annealed Kynar ARC and FMA coated bronze panels during weathering. The brightness was increased by $10 \%$ for photos of plain samples. 


\section{Appendix F: Additional surface characterization of clays, PNCs, and their properties}

\section{Raman spectroscopy, Wide-Angle X-Ray Diffraction and Thermo-Gravimetric Analysis}

The Raman spectral features of LAPC and LFPc compared to unmodified clay (LNa) each have a distinctive characteristic vibrations in Figure $\mathrm{F}_{1}$ confirming their presence: (1) a carbonyl stretching band at $1718 \mathrm{~cm}^{-1}(v C=0)$ for LAPc or (2) the trifluoromethyl mode at $749 \mathrm{~cm}^{-1}\left(v C-\mathrm{F}_{3}\right)$ for LFPc. Other characteristic frequencies include aliphatic vibrations from methylene asymmetric $\left(v_{a s} \mathrm{C}-\mathrm{H}_{2}\right)$ and symmetric $\left(v_{\mathrm{s}} \mathrm{C}-\mathrm{H}_{2}\right)$ stretching at 2945 and $2900 \mathrm{~cm}^{-1}$, where the intensities of these bands were blue shifted for LFPc due to the dense electronegativity of the fluorinated tail $\left(v_{a s} C-H_{2}=2955\right.$ and $v_{s} C-H_{2}=2920$ $\mathrm{cm}^{-1}$ ). In-chain methylene motions are responsible for a number of $\mathrm{C}-\mathrm{H}$ deformation modes present from $1462-1221 \mathrm{~cm}^{-1}$. Peaks indicative of the tetrahedral structure of the silicate include the unmodified clay LNa's characteristic peak at $683 \mathrm{~cm}^{-1}\left(\mathrm{Si}_{-} \mathrm{O}_{4}\right.$ stretch) and various metal-oxide vibrations found lower than $600 \mathrm{~cm}^{-1}$ including peaks at 482 $\mathrm{cm}^{-1}(\mathrm{Si}-\mathrm{O})$ and $360 \mathrm{~cm}^{-1}(\mathrm{Mg}-\mathrm{O})$.

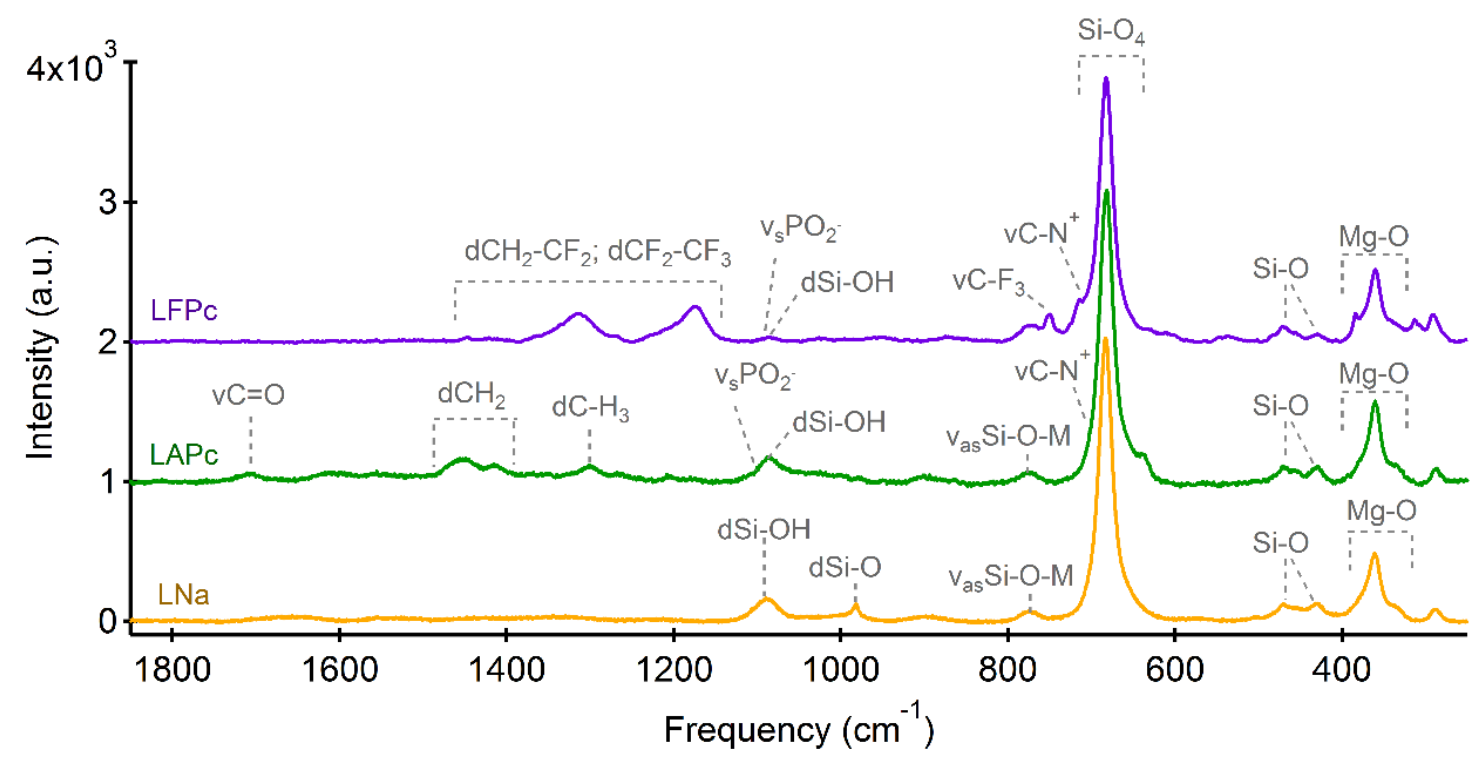

Figure F1. Raman spectra of functionalized clays, where $\mathrm{M}-\mathrm{OH}$ denotes the $\mathrm{Mg}-\mathrm{OH}$ and $\mathrm{Si}-\mathrm{OH}$ stretching vibrations. 
The hydroxyl region of the nano-clays $\left(4000-2800 \mathrm{~cm}^{-1}\right)$ was described in the main text by Figure 6.3 and the hydrophilicity ratios discussed in Section 3.1 can be found in Table S1. The spectra of LA and LF (spectra not shown) contained a peak at $832 \mathrm{~cm}^{-1}$ corresponding to an out-of-plane $\mathrm{C}-\mathrm{H}$ deformation band indicative of unreacted silane linkages and upon cation exchange these bands disappeared. We have previously reported similar observations from FTIR spectra of these materials. ${ }^{54}$

The wide-angle diffraction (WAXD) patterns in Figure F2 of dry Laponite before and after modification showed an increase in the interlayer gallery or basal spacing $\left(\mathrm{d}_{001}\right)$ of the clay upon functionalization. Sodium Laponite is an amorphous nano-clay and, without modification, exists as an average of 3 stacked platelets in its bulk form with no long-range order. As such, the WAXD spectra show broad peaks at most angles that subsequently mask some of the characteristic ool patterns, such as $d_{002}$ and $d_{004}$. The broad $d_{001}$ of the LNa peak at $6.972 \theta$ indicate: (1) random disordered stacking of the clay and (2) expansion of the gallery space by $\sim 0.3 \mathrm{~nm}$, the tumbling radius of water, suggesting the presence of a layer of water trapped between platelets.

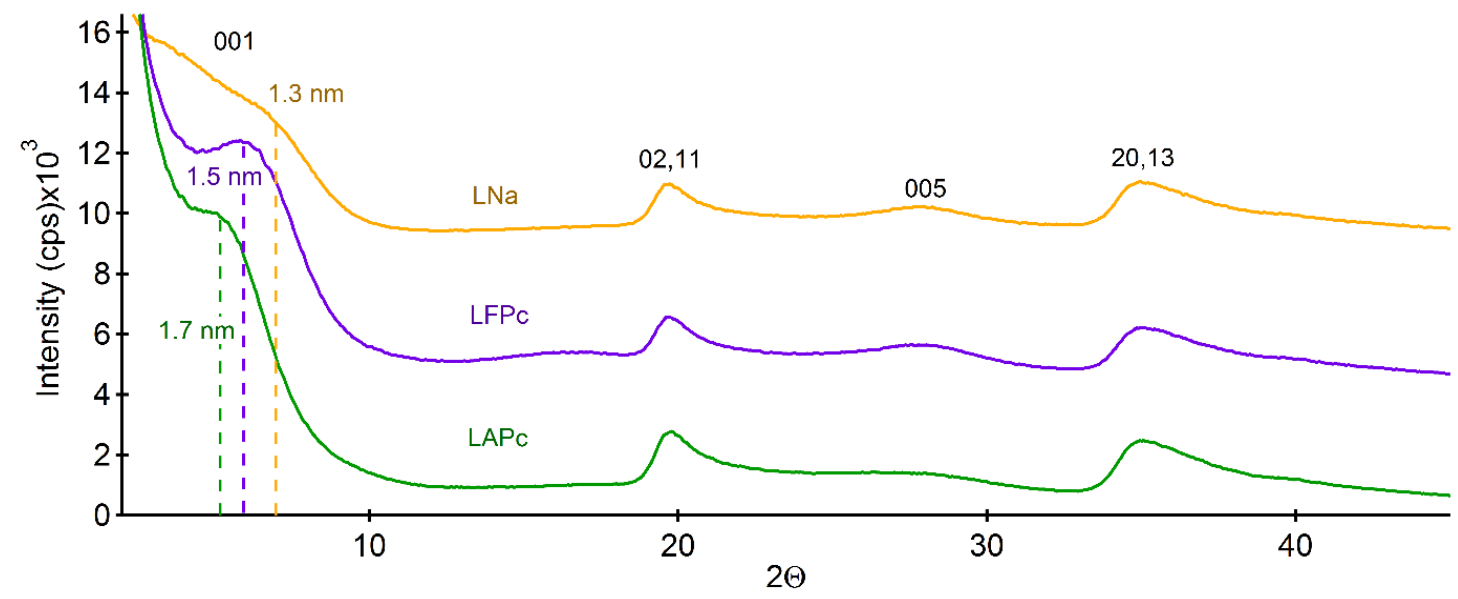

Figure F2. WAXD spectra of unmodified clay (LNa, yellow) and modified clays LFPc (purple) and LAPC (green) with the $d_{o 01}$ basal spacing in nanometers and notated with a dashed line.

Modification of clays expanded the gallery space (observed by a shift of doo1 to lower angles) to $1.74 \mathrm{~nm}$ for LAPc and $1.51 \mathrm{~nm}$ for LFPc, as listed in Table F1 with their net change. Given the lateral cross-section of the siloxane is close to $0.5 \mathrm{~nm}$, the basal expansion is likely related to the lateral height of excess silane intercalated between 
platelets in a monolayer fashion where both ends are touching the clay surface. Furthermore, an interdigitated bilayer arrangement of Pc in the gallery space would have an expansion greater than the length of the cationic chain, $0.61 \mathrm{~nm}$, as shown by LAPC. Typically this expansion bears little influence on the particle's behavior in a solvent. ${ }^{188}$ It is of note when initially dispersed in water, organo-clays LA and LF did not exfoliate fully until blended with the same peptizing agent as LNa (tetrasodium pyrophosphate, TSPP). Lack of exfoliation and expansion of the gallery space led us to consider silane attachment to multiple clay platelets as others have reported. ${ }^{172}$ However, we consider this possibility to be less likely due to steric hindrance of the siloxane since the distance between oxygen atoms on the silicon head group can be as much as $0.3 \mathrm{~nm}$.

Table F1. Physical properties of clays described by Raman, WAXD, and TGA

\begin{tabular}{|c|c|c|c|c|c|c|c|}
\hline & $\begin{array}{l}\text { Free } \mathrm{H}_{2} \mathrm{O} \\
3420 \mathrm{~cm}^{-1}\end{array}$ & $\begin{array}{l}\mathrm{Si}-\mathrm{O}_{4} \\
683 \mathrm{~cm}^{-1}\end{array}$ & $\frac{13450 \mathrm{~cm}^{-1}}{1683 \mathrm{~cm}^{-1}}$ & $2 \theta\left(^{\circ}\right)$ & $\mathrm{d}_{\mathrm{oo1}}(\mathrm{nm})$ & $\Delta \mathrm{d}_{\mathrm{OO1}}(\mathrm{nm})$ & $\begin{array}{l}\text { Adsorbed } \mathrm{H}_{2} \mathrm{O} \\
(\% \mathrm{w} / \mathrm{w})\end{array}$ \\
\hline LNa & 528.5 & 1416.9 & 0.373 & 6.97 & 1.27 & 0.35 & 12.1 \\
\hline LAPC & 819.2 & 2805.5 & 0.292 & 5.1 & 1.74 & 0.82 & 6.45 \\
\hline LFPc & 578.2 & 3027.2 & 0.191 & 5.84 & 1.51 & 0.59 & 7.00 \\
\hline
\end{tabular}

Raman spectra were normalized to value of $\mathrm{Si}_{-} \mathrm{O}_{4}$ band at $683 \mathrm{~cm}^{-1}$. The increase observed in $d_{001}$ spacing was calculated relative to the dry unit cell $(0.92 \mathrm{~nm})$

Thermal gravimetric analysis of covalently modified clays LA and LF was compared to unmodified clay LNa. Thermograms by mass loss and their first derivative plots are shown in Figure $\mathrm{F}_{3}$ where three main phases of degradation upon heating of LNa can be observed. These phases indicate: (1) loss of adsorbed water on the particle surface from $50-330^{\circ} \mathrm{C}$ and equal to $12.1 \%$ mass, (2) loss of interlayer from $330-600{ }^{\circ} \mathrm{C}$ where approximately $2.5 \%$ mass loss occurred and (3) the onset of hydroxyl degradation at $600{ }^{\circ} \mathrm{C}$. Modified clays exhibited four main phases, the first of which from $50-170^{\circ} \mathrm{C}$ was also due to bulk water loss and indicated $6.5 \%$ and $7 \%$ water content in the clay structure for LA and LF, respectively. This was less water than released by unmodified clay $\mathrm{LNa}$, as compared in Table $\mathrm{F} 1$. A decrease in hydrophilicity 
after modification should correspond to a decrease in surface charge and was confirmed by zeta potential measurements listed in Table F2. The calculated number of surface-bound water molecules per Laponite unit cell (MpU=\#molecules/\# clay unit cell) was 7.1 MpU for $L N a$, 5.1 MpU for $L A$, and 4.5 MpU for LF. The second decomposition phase in LA and LF from $150-320^{\circ} \mathrm{C}$ indicated a significant amount of silane was adsorbed to the surface of the clay due the lower temperature of degradation than the covalently bound molecules observed in the third region from $320-600{ }^{\circ} \mathrm{C}$. Using the mass lost in each phase, relative percentage of molecules adsorbed as opposed to covalently bound were $23 \%$ for LA and $35 \%$ for LF.
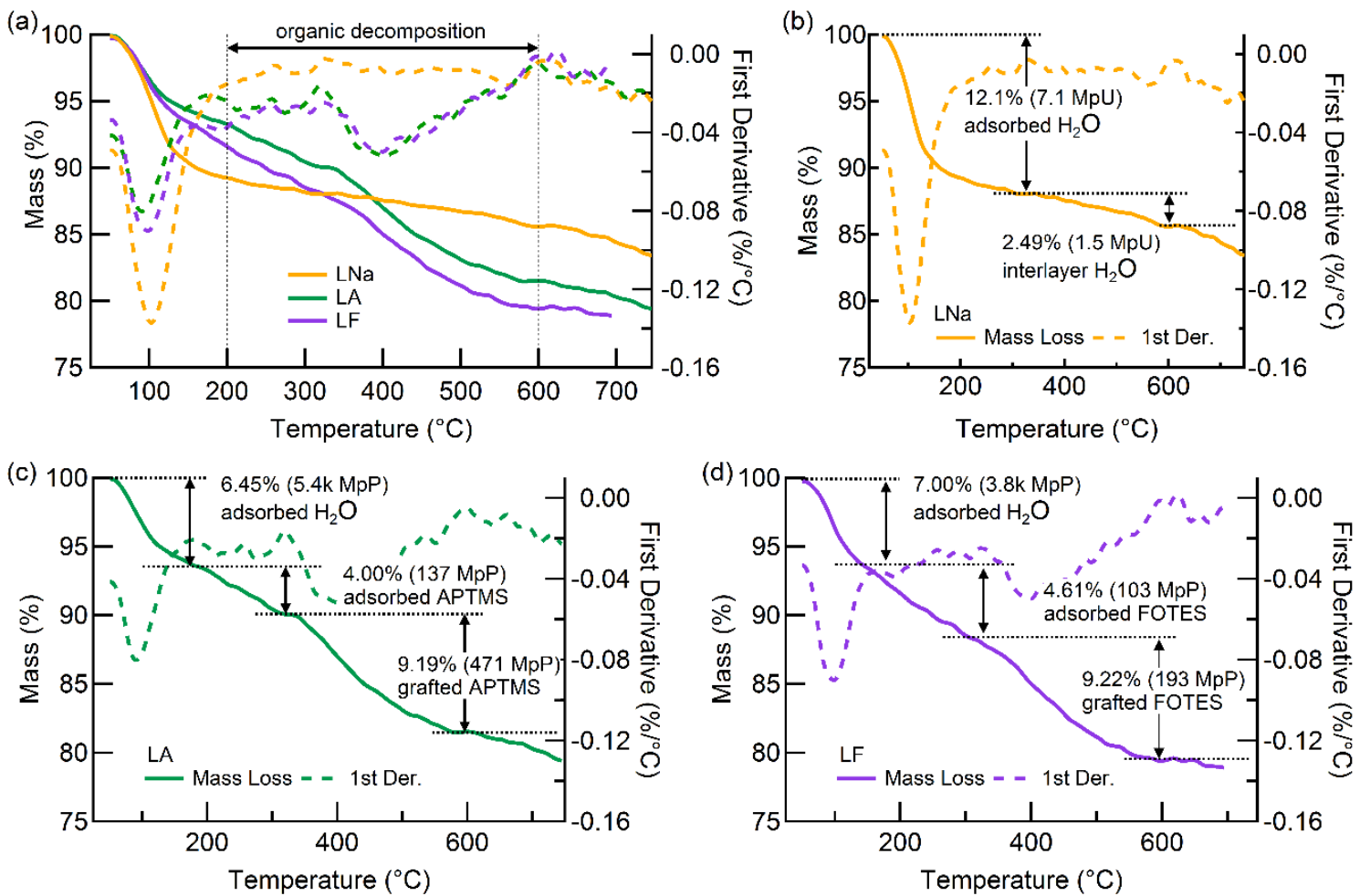

Figure $F_{3}$. TGA profiles of organically modified (LA and LF) versus unmodified LNa clay

These data combined provide insight into the physical changes the clays undergo from modification. From thickness and unit cell values obtained during WAXD analysis, it was estimated that there are 1,050 unit cells per Laponite platelet. This number compared well to other works that used surface adsorption and titrations to 
derive an accessible hydroxyl density of $0.36 \mathrm{mmol}-\mathrm{OH} / \mathrm{g}$ clay. ${ }^{171}$ Given dry conditions of the synthesis, platelets were likely suspended in small stacks of 3 discs in anhydrous toluene with broken edge hydroxyls available for grafting. Utilizing the measured coordinates by XRD in combination with the empirical formula of the unit cell, each 3disc stack would have approximately 730 silanols available for attachment along the disc edges and thermal gravimetry data provided a value of 907 accessible hydroxyl groups.

\section{Small-Angle X-Ray Scattering and Thin-Film X-Ray Diffraction}

Small-angle scattering is a useful technique for modeling the shape and size of nanoparticles in various media. Using the values obtained in WAXD as initial estimates, improved fitting of the scattering form factor used a single dispersion of cylinder shaped particles with an aspect ratio of 0.05 to provide the values listed in Table F2. The model-fit diameter of $\mathrm{LNa}$ in Figure $\mathrm{F}_{4}$ a was found to be $22.6 \mathrm{~nm}$ with a corresponding layer thickness of $1.13 \mathrm{~nm}$, nearest neighbor at $15.9 \mathrm{~nm}$ and a narrow polydispersity $(R S D=14.3 \%)$. The decreased layer thickness and large nearest neighbor value suggested the clay particles were completely exfoliated in water, while a small RSD showed uniform particle size dispersion.

Table F2. Physical properties of clays in water - SAXS

\begin{tabular}{llllll}
\hline & $\mathrm{SAXS} \mathrm{dH} 2 \mathrm{O}(\mathrm{nm})$ & $\mathrm{SAXS} \mathrm{tH} 2 \mathrm{O}(\mathrm{nm})$ & neighbor $(\mathrm{nm})$ & $\mathrm{RSD}(\%)$ & \%Error \\
\hline $\mathrm{LNa}$ & 22.6 & 1.13 & 15.9 & 14.3 & 0.16 \\
\hline $\mathrm{LAPC}$ & 33.6 & 1.68 & 1.68 & 47.0 & 0.28 \\
\hline $\mathrm{LFPC}$ & 30.1 & 1.51 & 1.81 & 25.7 & 0.50 \\
\hline
\end{tabular}

Analysis was performed for aqueous clay suspensions with a cylinder geometry (aspect ratio $=0.05$ )

Further inspection of undiluted aqueous dispersion of covalently modified clays by SAXS indicated good correlation to WAXD values with some degree of particle aggregation at the deliverable concentration $(2.5 \% \mathrm{w} / \mathrm{w})$. Dispersions were termed 'exfoliated' or 'flocculated' as dependent on the similarity of the SAXS models to unmodified $\mathrm{LNa}$, which has been well documented to form a stable exfoliated claywater system at low concentrations. ${ }^{191}$ The average diameter obtained from a cylinder 
model fit to the scattering profile of aqueous LAPc was equal to $34.8 \mathrm{~nm}\left(\mathbf{t}_{\mathrm{H}_{2} \mathrm{O}}=1.74\right.$ $\mathrm{nm})$ and $30.2 \mathrm{~nm}$ for LFPc $\left(\mathbf{t}_{\mathrm{H}_{2} \mathrm{O}}=1.51 \mathrm{~nm}\right)$. All aqueous dispersions of modified clays showed a closer nearest neighbor than unmodified $\mathrm{LNa}$, where the furthest particle distance belonged to LFPC $(1.81 \mathrm{~nm})$ and the most particle interaction by LAPC (1.67 $\mathrm{nm})$. For LAPc the nearest neighbor values showed the closest platelet was within the distance traveled perpendicularly through one hydrated particle, indicative of short range ordering in solution. For $\mathrm{LNa}$ and LFPc, with the nearest neighbor values being larger than the layer thickness of aqueous clay, which suggests that swollen, hydrated platelets were mostly exfoliated. Increased particle-particle interactions were seen for LFPc by a small peak at $0.3 \AA$.
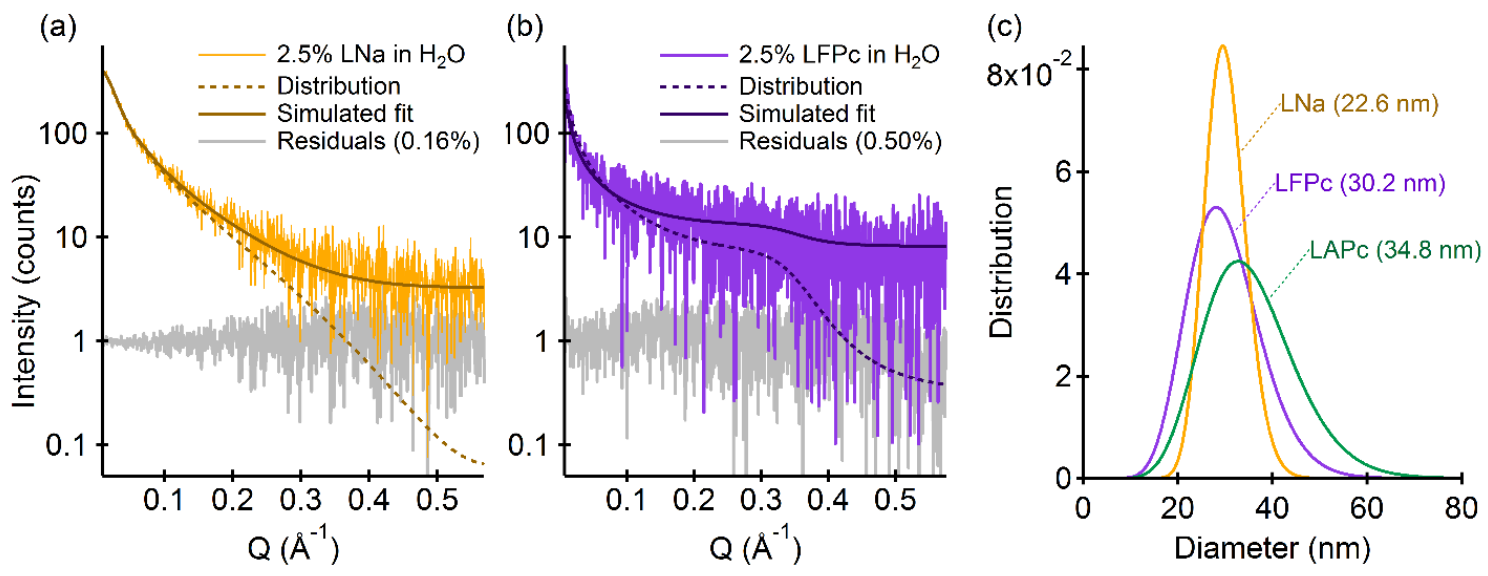

Figure F4. Small-angle scattering spectra of functionalized Laponite in aqueous colloidal suspensions $(2.6 \%)$

The modeled distributions also provided evidence for some larger aggregates but the majority of particles were near the median values, as shown in Figure $\mathrm{F}_{4} \mathrm{C}$. If using the RSD\% (relative size distribution) as a marker of polydispersity, cationic exchange with phosphoryl choline increased polydispersity of LFPc to $25.7 \%$. However, this value was still lower than LAPC (RSD=47.0\%) and weighted to larger, not smaller, diameters of 50-90 $\mathrm{nm}$. The steric bulk of the fluorinated silane may act to repel particles after anisotropic (charge disparity) behavior is restored by cationic exchange. 
Whereas, the smaller APTMS chains could allow for tethering through hydrogen bonding (dimerization of end groups) and possible edge-face aggregation if surface coverage was not uniform.

Surface analysis by ATR-FTIR of weathered composites in Figure 6.8 showed significant erosion of the acrylic component of the latex coating with simultaneous enrichment of all PVDF associated vibrations. Coating surfaces are particularly sensitive to photdegradation and typically a loss of film thickness during weathering corresponds to a simultaneous decrease in barrier properties. In Figure 6.7 we observed an increase in barrier properties over time, up to 3500 hours in the case of LFPc, even after the film had lost substantial thickness. Since PVDF is well known to have a temperaturedependent crystalline structure, ${ }^{190,}{ }^{192}$ it was hypothesized that an increase in the number of impenetrable crystalline domains had occurred at the PVDF-rich surface of the film, ultimately increasing barrier properties.

Thin-film XRD spectra were obtained to investigate both the amount and quality of the composite films remaining after 3500 of weathering. The characteristic diffraction peaks of PVDF are shown for a weathered composite containing LF in Figure F5b, where reflections of the TGTG' form of PVDF ${ }^{7}$ produced peaks at $18.7^{\circ}, 19.8^{\circ}$, and $26.5^{\circ} 2 \Theta$. Since only an amorphous scattering halo can be clearly observed for PVDF (non-weathered), any sharpening of the aforementioned peaks would indicate an increase in the number and uniformity of PVDF crystalline domains. These same peaks were also present in spectra acquired using a thin-film geometry, which allowed for an in-situ investigation of the entire bulk film. A decreased intensity for each sample spectrum upon weathering confirmed loss of film thickness and sharpening of the $d_{110}$ peak at $18.72 \Theta$ confirmed increased PVDF crystallinity. The grazing-incidence data shown in Figure $\mathrm{F}_{5} \mathrm{~b}$ was acquired in a surface-sensitive geometry, and showed less overlap between the non-weathered and weathered films, also an indication of an increase in crystalline PVDF at the surface of the coating. Furthermore, the appearance of low-angle peaks at 4.2 and $6.32 \Theta$ correspond to the $d_{001}$ spacings of Laponite, as discussed in previous sections. While these types of peaks would typically be unwanted 
in a composite (they indicate long-range stacking of pillars, as opposed to exfoliated discoids), the LF composite was the only film that showed diffraction peaks of nanoclay in-situ at any point in this study. Appearance of these peaks for the sample with LF as opposed to the other films indicated surface enrichment of not only PVDF but also the nano-caly, as chemical modification with FOTES may have increased particle retention in the coating.

(a) Thin-Film XRD

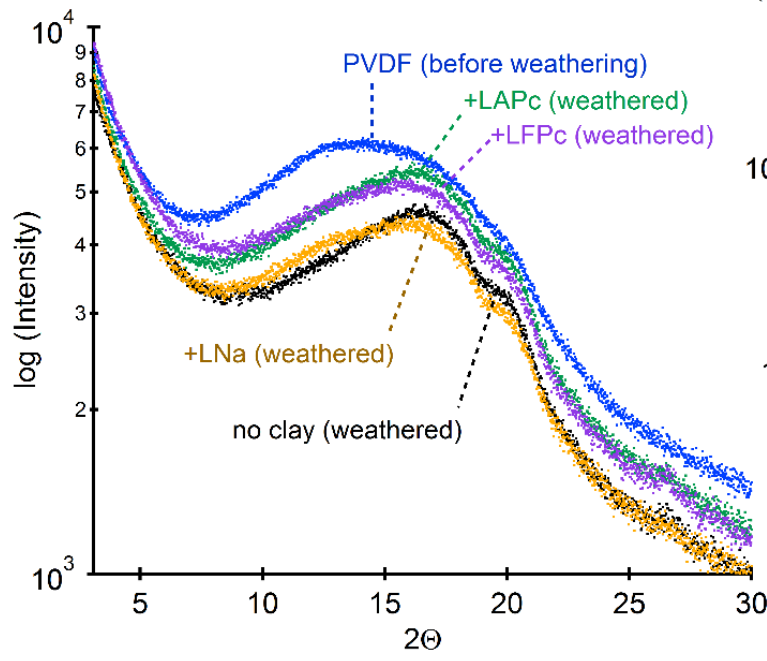

(b) $0.5^{\circ}$ Grazing-Incidence XRD

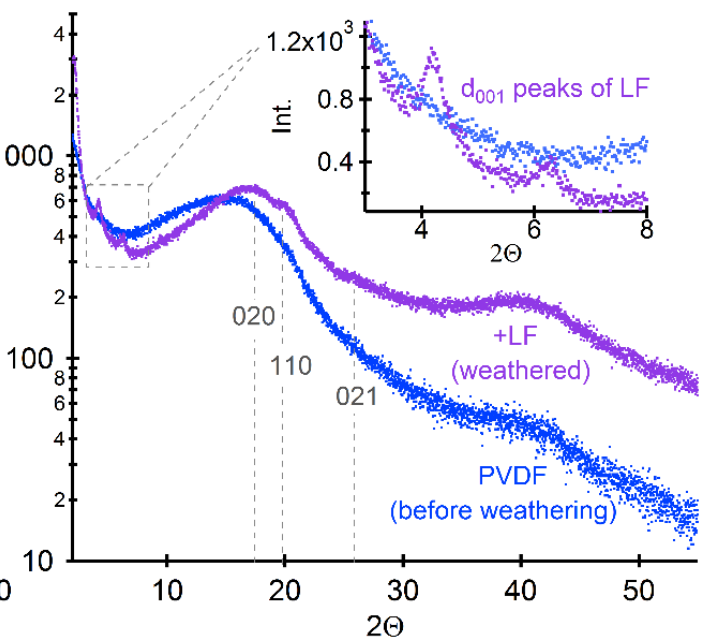

Figure F5. Diffraction spectra of PVDF-Laponite composites weathered by QUV for 3500 hours with (a) Thin-Film spectra showing increased PVDF crystallinity at 18.7 and $19.82 \Theta$ after weathering and (b) Grazing Incidence $\left(0.5^{\circ}\right)$ of non-weathered PVDF compared to PVDF+LF showing both surface enrichment of modified clay LF with doo1 spacings at $4.2^{\circ}$ and $6.3^{\circ} 2 \Theta$ as well as increased PVDF crystallinity with reflections at $18.7^{\circ}$ (do20), $19.8^{\circ}$ (d110), and $26.5^{\circ}$ (do21) $2 \Theta$.

\section{Circuit element trends in imepdance analysis of PNC's}

All EIS spectra acquired of initial films before and during weathering were fit to a circuit model with two CPE/Resistor pairs in parallel: $C P E_{\text {coat }}\left(R_{\text {pore }}\right) C P E_{d l}\left(R_{c t}\right)$ until failure expanded the circuit model with another pair. The equivalent for a failed coating is $C P E_{\text {perc }}\left(R_{\text {perc }}\right) C P E_{\text {coat }}\left(R_{\text {pore }}\right) C P E_{\text {dll }}\left(R_{c t}\right)$ and was only observed for PVDF without clay and with LNa after 3500 hours of QUV weathering. Additionally all EIS spectra were normalized with the cell constant $\left(k=14.62 \mathrm{~cm}^{2}\right)$ so that all values presented with units in Farads $(F)$ are $F / \mathrm{cm}^{2}$ and Ohms $(\Omega)$ are $\Omega \cdot \mathrm{cm}^{2}$. 
The capacitance of the protective film can be thought of as a material's ability to store charge across a select area. A capacitor in parallel with a resistor representing the coating generally corresponds to the coating capacitance, $C_{\text {coat, }}$ of the entire film and the pore resistance, $R_{\text {pore, }}$ or the average open path through the coating to the substrate. Thus the product of the two elements is the time constant, $T_{\text {coat }}=R_{\text {pore }} C_{\text {coat }}$ and represents the shortest (minimum) relaxation time for an ion to travel from the bulk solution through the coating to the metal interface. The high frequency $C P E(R)$ pair dominates the EIS spectra of these films from approximately $1-10^{6} \mathrm{~Hz}$ and therefore $C_{\text {coat, }} R_{\text {pore, }}$ and $T_{\text {coat }}$ can be assumed to represent the bulk of the protective coating. Based on the general formula for a planar capacitor (Equation A6.1), capacitance can increase due to: (1) change in permittivity from increased porosity, (2) change in permittivity from uptake of higher $\varepsilon$ liquid into the material matrix, (3) increase in surface area of the plate, and (4) decrease in film thickness - distance between plates.

$C=\frac{\varepsilon_{o} \varepsilon_{m} A}{d}$

Although the data was fit with models containing constant phase elements in place of capacitors, all values were converted to effective capacitance in Farad using the Brugg equation (Eq. A6.2):

$C=\frac{(C P E \times R)^{\frac{1}{a}}}{R}$

\section{Coating capacitance}

An evolution of $C_{\text {coat }}$ can be observed in Figure $6.7 c$, where each coating experienced a decrease in capacitance after the first weathering cycle. Capacitance of films with modified clay materials were higher after exchange with phosphoryl choline, as expected from the hydrophilic nature of the cation and increased water sorption seen in the Raman data. After 1000 hours of weathering, all films containing clay began to increase in capacitance. The highest final $C_{\text {coat }}$ after $4500 \mathrm{~h}$ of QUV-B was observed for the PVDF film without clay, $1.78 \mathrm{nF}$, followed by $0.794 \mathrm{nF}$ for LAPc, $0.684 \mathrm{pF}$ for $\mathrm{LNa}$, 
and the lowest was $0.427 \mathrm{pF}$ for LFPc. Recalling the ways in which a capacitor can increase in value, the most likey contributions after weathering include decreasing film thickness and increased permittivity, as the area is relatively unchanged and the films were analyzed after a UV exposure cycle where little water remained in the film. Changes in film thickness were measured with a tri-gloss meter, and the largest decrease was observed for the coatings with the highest capacitance: PVDF lost $37 \%$ of its total film thickness and LAPc lost $43 \%$. In contrast, the coating with LFPc had the lowest capacitance and underwent the smallest change in film thickness of $-23 \%$ and similarly LNa lost $29.4 \%$. Evidence of surface ablation with loss of acrylic functionality in the film was also observed by ATR-FTIR and TF-XRD.

\section{Film Resistance}

The term film resistance is useful to describe a film having number of pore channels that ions may travel through more quickly than they may through a film lacking such pores. The resistance of the full intact film ( $\left.R_{\text {coat }}\right)$ is assumed to have a value much greater than the pore resistance and is not typically observed by experimental methods. Plotted for each coating over time in Figure 6.7, by 2000 hours of QUV-B weathering $R_{\text {pore }}$ had decreased from its maximum value in all composites. Coatings without clay and those with LNa showed partitioning of the film at advanced weathering times which was represented by a new time constant for a percolation layer, as shown in Figure $6.7 \mathrm{~d}$. The final film resistances after 4500 hours OUV-B weathering were highest for the coating with LFPc $\left(R_{\text {pore }}=442 \mathrm{M} \Omega\right)$, followed by LAPC $\left(R_{\text {pore }}=87.1 \mathrm{M} \Omega\right)$, then $L N a\left(R_{\text {perc }}=27.4 \mathrm{M} \Omega\right.$ and $\left.R_{\text {pore }}=183 \mathrm{M} \Omega\right)$ and latex resin without nano-clay $\left(R_{\text {perc }}=0.373 \mathrm{M} \Omega\right.$ and $R_{\text {pore }}=1.85 \mathrm{M} \Omega$ ). The film with LFPc maintained a film resistance above $1 \mathrm{G} \Omega$ after 3500 hours of weathering and as thus was considered exceptional protection.

In general, it can be assumed that the resistance of the film drops as ion transport rises due to chemical degradation of the film (increased material permittivity). Resistance is not only related to a material's ability to transport charge, 
but also directly proportional to path length (here film thickness) and inversely proportional to area (here working electrode area). The observed decreases in film thickness may explain the later drop in $R_{\text {pore }}$ upon extended weathering, but this is in direct opposition to the initial observed increases up until 1500 hours. Accelerated weathering methods typically have a long UV step at an elevated temperature above the MFFT (here the UV step is run at $60^{\circ} \mathrm{C}$ when MFFT FMA $=12{ }^{\circ} \mathrm{C}$ ) and we hypothesize that these steps serve as continued annealing to provide limited repair for films retaining their flexibility throughout weathering. This maintained annealing drives out water from the film, promotes close packing and limited reorganization, all which decreased permittivity of the coatings.

\section{Time constants}

The combined product of capacitance and resistance of a protective coating can also be considered the relaxation time of the film after polarization and provides an average time for charge transport through the film to the metal surface. The initial time constant of the films studied here did not follow the same trend as $C_{\text {coat }}$ nor $R_{\text {pore }}$ and are ordered from shortest to longest: PVDF without clay (2.2 ms), LAPc (33.0 ms), LNa (144.7 ms), and LFPC (185.5 ms). After 4500 hours of weathering, $T_{\text {coat }}$ decreased and is ordered from shortest to longest as follows: PVDF (0.0059 ms), LNa (18.7 ms), LAPC (69.2 ms), and LFPc (188.7 ms). Given the very small capacitances of these films in the pico- to nano- Farad range, the magnitude of the resistive components have a greater contribution in determining the time and is thus more closely related to the permittivity (or resistivity or in-phase behavior) of the dielectric being studied.

\section{References}

1. Clare, T. L.; Swartz, N. A., Characterization of high performance protective coatings for use on culturally significant works. In Intelligent Coatings for Corrosion Control, Tiwari, A.; Hihara, L.; Rawlins, J., Eds. Butterworth-Heinemann: Elsevier, 2014; pp 641-671.

2. Jung, H.; Kim, H.-M.; Choy, Y. B.; Hwang, S.-J.; Choy, J.-H., Laponite-based nanohybrid for enhanced solubility and controlled release of itraconazole. International Journal of Pharmaceutics 2008, 349, (12), 283-290. 
3. Herrera, N. N.; Letoffe, J.-M.; Putaux, J.-L.; David, L.; Bourgeat-Lami, E., Aqueous Dispersions of Silane-Functionalized Laponite Clay Platelets. A First Step toward the Elaboration of Water-Based Polymer/Clay Nanocomposites. Langmuir 2004, 20, (5), 1564-1571.

4. Herrera, N. N.; Letoffe, J. M.; Reymond, J. P.; Bourgeat-Lami, E., Silylation of laponite clay particles with monofunctional and trifunctional vinyl alkoxysilanes. Journal of Materials Chemistry 2005, 15, (8), $863-871$.

5. Kimura, H.; Sakurai, M.; Sugiyama, T.; Tsuchida, A.; Okubo, T.; Masuko, T., Dispersion state and rheology of hectorite particles in water over a broad range of salt and particle concentrations. Rheologica Acta 2011, 50, (2), 159-168.

6. Horibe, H.; Hosokawa, Y.; Oshiro, H.; Sasaki, Y.; Takahashi, S.; Kono, A.; Nishiyama, T.; Danno, T., Effect of heat-treatment temperature after polymer melt and blending ratio on the crystalline structure of PVDF in a PVDF/PMMA blend. Polym J 2013, 45, (12), 1195-1201.

7. Horibe, H.; Sasaki, Y.; Oshiro, H.; Hosokawa, Y.; Kono, A.; Takahashi, S.; Nishiyama, T., Quantification of the solvent evaporation rate during the production of three PVDF crystalline structure types by solvent casting. Polym J 2014, 46, (2), 104-110. 